\title{
Atypical cortical connectivity in autism spectrum disorder (ASD) as measured by magnetoencephalography (MEG)
}

\author{
Robert Alexander Seymour \\ BSc, MSc
}

MACQUARIE UNIVERSITY

Department of Cognitive Science

Faculty of Human Sciences Macquarie University, Sydney, Australia

This dissertation is presented for the degree of Doctor of Philosophy (PhD), February 2019 


\section{Table of Contents}

$\begin{array}{lr}\text { Summary } & 5\end{array}$

$\begin{array}{lr}\text { Statement of Originality } & 6\end{array}$

$\begin{array}{ll}\text { Acknowledgments } & 7\end{array}$

$\begin{array}{lc}\text { Publications } & 8\end{array}$

$\begin{array}{lr}\text { Chapter 1: General Introduction } & 9\end{array}$

1.1 Autism Spectrum Disorder (ASD) 9

$\begin{array}{ll}1.2 \text { Neurocognitive Models of ASD } & 10\end{array}$

$\begin{array}{ll}1.3 \text { Neurophysiological models of ASD } & 11\end{array}$

1.4 "Oscillopathies" and atypical connectivity in ASD 12

1.5 A Novel approach to ASD: local dysregulation, global 19

hypoconnectivity, and deficient predictive coding

1.6 Thesis Aims and Outline $\quad 22$

Chapter 2: The Detection of Phase Amplitude Coupling

During Sensory Processing 25

2.1 Abstract 25

2.2 Introduction 26

2.3 Materials and Methods $\quad 28$

2.4 Results 36

2.5 Discussion $\quad 42$

Chapter 3: Dysregulated Oscillatory Connectivity in the Visual System in Autism Spectrum Disorder 46

3.1 Abstract $\quad 46$

3.2 Introduction $\quad 47$

3.3 Materials and Methods $\quad 50$

3.4 Results $\quad 56$

3.5 Discussion 64

Chapter 4: Reduced Auditory Steady State Responses

in Autism Spectrum Disorder $\quad 69$

4.1 Abstract $\quad 69$

$\begin{array}{ll}4.2 \text { Introduction } & 70\end{array}$

$\begin{array}{ll}\text { 4.3 Materials and Methods } & 72\end{array}$

$\begin{array}{ll}4.4 \text { Results } & 78\end{array}$ 
Chapter 5: Testing Predictive Coding Theories of Autism Spectrum Disorder Using the Auditory Oddball Paradigm - An MEG Study

5.1 Abstract $\quad 92$

5.2 Introduction 93

5.3 Materials and Methods 96

$\begin{array}{ll}5.4 \text { Results } & 102\end{array}$

$\begin{array}{ll}5.5 \text { Discussion } & 111\end{array}$

Chapter 6: The Neural Basis of Perspective-Taking in $\begin{array}{ll}\text { Neurotypical and Autistic Populations } & 115\end{array}$

6.1 Abstract 115

$\begin{array}{ll}6.2 \text { Introduction } & 116\end{array}$

6.3 Materials and Methods 120

$\begin{array}{ll}6.4 \text { Results - Experiment } 1 & 127\end{array}$

6.5 Results - Experiment $2 \quad 132$

$\begin{array}{ll}6.6 \text { Discussion } & 139\end{array}$

$\begin{array}{lr}\text { Chapter 7: General Discussion } & 147\end{array}$

$\begin{array}{ll}\text { 7.1 Summary of Main Findings } & 147\end{array}$

$\begin{array}{ll}7.2 \text { Limitations } & 150\end{array}$

7.3 Implications and future directions 153

$\begin{array}{ll}7.4 \text { Conclusion } & 163\end{array}$

$\begin{array}{lr}\text { References } & 164\end{array}$

$\begin{array}{lr}\text { Appendices } & 185\end{array}$

$\begin{array}{lr}\text { Ethics Approval } & 190\end{array}$ 


\title{
Summary
}

\section{Atypical cortical connectivity in autism spectrum disorder (ASD) as measured by magnetoencephalography (MEG)}

\author{
Robert Alexander Seymour
}

\author{
2019
}

\begin{abstract}
Autism Spectrum Disorder (ASD) is a neurodevelopmental condition, characterised by impairments in social interaction and communication, the presence of repetitive behaviours, and multisensory hyper- and hypo-sensitives. This thesis utilised magnetoencephalography, in combination with robust analysis techniques, to investigate the neural basis of ASD. Based on previous research, it was hypothesised that cortical activity in ASD would be associated with disruptions to oscillatory synchronisation during sensory processing, as well as during high-level perspective-taking. More specifically, a novel framework was introduced, based on local gamma-band dysregulation, global hypoconnectivity and deficient predictive-coding. To test this framework, data were collected from adolescents diagnosed with ASD and age-matched controls.
\end{abstract}

Using a visual grating stimulus, it was found that in primary visual cortex, ASD participants had reduced coupling between the phase of alpha oscillations and the amplitude of gamma oscillations (i.e. phase amplitude coupling), suggesting dysregulated visual gamma in ASD. These findings were based on a robust analysis pipeline outlined in Chapter 2. Next, directed connectivity in the visual system was quantified using Granger causality. Compared with controls, ASD participants showed reductions in feedback connectivity, mediated by alpha oscillations, but no differences in inter-regional feedforward connectivity, mediated by gamma oscillations. In the auditory domain, it was found that ASD participants had reduced steady-state responses at $40 \mathrm{~Hz}$, in terms of oscillatory power and inter-trial coherence, again suggesting dysregulated gamma. Investigating predictive-coding theories of ASD using an auditory oddball paradigm, it was found that evoked responses to the omission of an expected tone were reduced for ASD participants. Finally, we found reductions in theta-band oscillatory power and connectivity for ASD participants, during embodied perspective-taking. Overall, these findings fit the proposed framework, and demonstrate that cortical activity in ASD is characterised by disruptions to oscillatory synchronisation, at the local and global scales, during both sensory processing and higher-level perspective-taking.

Keywords: Autism Spectrum Disorder; Magnetoencephalography; Oscillations; Phase Amplitude Coupling; Connectivity. 


\section{Statement of Originality}

This thesis is being submitted to Macquarie University and Aston University in accordance with the Cotutelle agreement dated 2016.

To the best of my knowledge and belief, the thesis contains no material previously published or written by another person except where due reference is made in the thesis itself.

The research presented in this thesis was approved by the Macquarie University Human Research Ethics Committee (reference number: 5201300843) and Aston University Research Ethics Committee (Application Number: 574).

Signed:

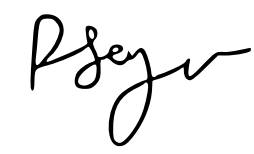

Date:

$8 / 2 / 19$ 


\section{Acknowledgements}

Firstly, I would like to thank my primary supervisors, Professor Klaus Kessler and Associate Professor Paul Sowman, co-supervisors, Professor Gina Rippon and Associate Professor Blake Johnson, and associate supervisor, Dr. Wei He. You have all been a constant source of guidance and encouragement during my PhD. I look forward to continuing working with you in the future.

A special mention to: Gerard Gooding-Williams who played an important role in data collection and participant recruitment; Dr. Jan-Mathijs Schoffelen and Dr. Hongfang Wang for providing MEG analysis guidance; and Dr. Jon Brock for organising the cotutelle agreement.

During my PhD, I have been helped by a team of talented support and technical staff at Aston and Macquarie Universities. I would like to extend a special thank you to: Lesley McKnight, Marcus Ockenden, Craig Richardson, Sian Worthen, Elisabeth Stylianou, Dr Stan Tarnavskii, and Caroline Brocklebank.

I have encountered some amazing people and made life-long friends over the past threeand-a-half years: Julien Millasseau, Diandra Brkić, Gloria Romagnoli, David Meng and Valerie Pu, to name but a few. You have made the journey so much fun, and the trials and tribulations of academic life, just that bit more bearable!

I would like to thank my parents, Karen and Des, and extended family for constantly supporting me in academic pursuits. You have always encouraged me to think critically, be inquisitive and work hard: this thesis definitely didn't write itself!

Thank you to my girlfriend, Misha, for your constant support, patience and encouragement. I am very lucky to have you and excited for what the future holds.

Finally I would like to thank my brother, Louis. You once asked me "what makes people different from each other?". I hope this thesis goes some way to answering that question. 


\section{Publications}

\section{Published Journal Articles}

Kessler, K., Seymour, R. A., \& Rippon, G. (2016). Brain oscillations and connectivity in autism spectrum disorders (ASD): new approaches to methodology, measurement and modelling. Neuroscience \& Biobehavioral Reviews, 71, 601-620.

Seymour, R. A., Rippon, G., \& Kessler, K. (2017). The Detection of Phase Amplitude Coupling during Sensory Processing. Frontiers in neuroscience, 11, 487.

Seymour, R. A., Wang, H., Rippon, G., \& Kessler, K. (2018). Oscillatory networks of highlevel mental alignment: A perspective-taking MEG study. Neurolmage, 177, 98-107.

\section{Articles Under Review}

Seymour, R. A., Rippon, G., Gooding-Williams, G., Schoffelen, J. M., \& Kessler, K. (2018). Dysregulated Oscillatory Connectivity in the Visual System in Autism Spectrum Disorder. bioRxiv, 440586.

Please note that these publications form the basis of certain sections within this thesis. Additional information is provided at the start of the relevant Chapters $(1,2,3,6)$. 


\section{Chapter 1: General Introduction}

Please note, the general structure of this chapter is based on the following published journal article:

Kessler, K., Seymour, R. A., \& Rippon, G. (2016). Brain oscillations and connectivity in autism spectrum disorders (ASD): new approaches to methodology, measurement and modelling. Neuroscience \& Biobehavioral Reviews, 71, 601-620.

\subsection{Autism Spectrum Disorder (ASD)}

Autism Spectrum Disorder (ASD) is a life-long neurodevelopmental condition, characterised by impairments in social interaction and communication, and the presence of repetitive patterns of behaviours, interests or activities (APA 2013). Recent estimates suggest that ASD affects around 1 in 59 children (Baio, 2014). Throughout this thesis, the term "Autism Spectrum Disorder" (ASD) will be used, in accordance with the Diagnostic and Statistical Manual Fifth Edition (APA, 2013), however we acknowledge that some autistic people prefer the term "Autism Spectrum Condition" (Kenny et al., 2016).

In addition to cognitive differences, over $90 \%$ of autistic individuals report atypical sensory responses to a variety of stimuli, across multiple sensory domains (visual, auditory, gustatory, olfactory, tactile and proprioceptive) (Leekam, Nieto, Libby, Wing, \& Gould, 2007; Robertson \& Simmons, 2013; Tomchek \& Dunn, 2007). These experiences are often described in terms of hyper-sensitivities, with more pronounced responses to certain stimuli which sometimes result in overwhelming and unpleasant sensations (BaronCohen, Ashwin, Ashwin, Tavassoli, \& Chakrabarti, 2009). Hypo-sensitivies are also reported, which can lead to self-stimulatory behaviours, such as rocking, hand-flapping and the repetition of words/phrases (Bogdashina, 2003). Sensory hypo/hyper-sensitivies can significantly interfere with activities of daily living for autistic people, especially in young children (Jones, Quigney, \& Huws, 2003). Behavioural research has also indicated that perceptual differences occur in participants diagnosed with ASD (Simmons et al., 2009). Findings from such research include: reduced binocular rivalry (Freyberg, Robertson, \& Baron-Cohen, 2015; Robertson, Kravitz, Freyberg, Baron-Cohen, \& Baker, 2013); atypical perception of global motion (Pellicano, Gibson, Maybery, Durkin, \& Badcock, 2005; Robertson et al., 2014); reduced multisensory binding, reviewed in Simon \& Wallace (2016); and increased performance on visual search tasks (O'Riordan, 2004). 
It is now believed that sensory symptoms are a significant constituent of ASD (APA, 2015), and could even provide early diagnostic markers (Baum, Stevenson, \& Wallace, 2015; Kessler, Seymour, \& Rippon, 2016; Roberts et al., 2010; Robertson \& BaronCohen, 2017). There is also emerging evidence that low-level differences in sensory perception can predict high-level cognitive differences in ASD, reviewed in Robertson \& Baron-Cohen (2017). This speaks to a common mechanism underlying both sensory and social differences in ASD (Kessler et al., 2016).

\subsection{Neurocognitive models of ASD}

\subsubsection{Weak central coherence/enhanced perceptual processing}

Findings in relation to the sensory and perceptual differences in ASD have inspired two neurocognitive models: the 'weak central coherence', WCC, (Happé \& Frith, 2006) and 'enhanced perceptual functioning', EPF, accounts (Mottron, Dawson, Soulieres, Hubert, \& Burack, 2006). The WCC account argues that perceptual processes involved with aggregating information into a coherent, global whole are perturbed in ASD (Happé, 2005). This is supported by evidence of atypical perception of global motion in ASD (Robertson et al., 2014), and reduced susceptibility to certain visual illusions (Dakin \& Frith, 2005). In contrast, the EPF argues that autistic perception is simply biased towards local detail, due to enhanced "perceptual operations" (Mottron et al., 2006), consistent with findings of superior visual search in ASD (O'Riordan, 2004).

However, the focus of these theories is an imbalance between local and global perception, and hence neither attempts to explain the breadth of sensory symptoms reported by autistic people (Robertson \& Baron-Cohen, 2017), nor do they explain cooccurring non-sensory symptoms.

\subsubsection{Deficient predictive coding}

More recently, it has been proposed that sensory differences in ASD can be explained through Bayesian or predictive coding models of perception (Pellicano \& Burr, 2012; Rao \& Ballard, 1999). Such models describe the cognitive processes undertaken by the brain in terms of Bayesian inference; essentially the brain can be thought of as a Bayesian machine which seeks to minimise the difference between prior expectations or "predictions" and incoming sensory information. Pellicano \& Burr (2012), and later Van Boxtel \& Lu (2013), argue that differences in autistic perception may be the result of "hypo-priors" - essentially weaker top-down predictions. Alternatively, it has been suggested that ASD is characterised by atypical context-sensitive weighting of predictions, 
i.e. precision. In other words, sensory processing in ASD is argued to be influenced by incoming information to a much greater extent than top-down contextual information (Lawson, Rees, \& Friston, 2014; Palmer, Lawson, \& Hohwy, 2017; Van de Cruys et al., 2014). Given that social stimuli are generally context dependent, requiring considerable top-down influence, predictive coding theories of ASD could also explain differences in social cognition (Sevgi, Diaconescu, Tittgemeyer, \& Schilbach, 2016). Indeed, Palmer, Seth, \& Hohwy (2015) proposed a mechanistic account of mentalising deficits in ASD through the same precision-weighting dysfunction thought to underlie perceptual differences. Whilst there is emerging behavioural evidence supporting predictive-coding theories of ASD (Goris et al., 2018; Lawson, Mathys, \& Rees, 2017), the neural evidence remains sparse. For this reason, we tested predictive-coding theories of ASD using an auditory oddball paradigm (Chapter 5).

\subsection{Neurophysiological models of ASD}

The search for the neural underpinnings of ASD has focussed on differences in the structure and function of the cerebral cortex, from the cellular level to whole-brain patterns of cortical connectivity.

\subsubsection{Excitation-inhibition in autism}

Early findings with regards to ASD pathology at the cellular level suggested that the condition might be characterised by an imbalance between excitation and inhibition (E:I) (Casanova, Buxhoeveden, \& Brown, 2002; Rubenstein \& Merzenich, 2003). In the human cerebral cortex, a delicate $\mathrm{E}: \mathrm{I}$ balance is achieved through the arrangement of around 80100 excitatory neurons into cortical minicolumns, each surrounded by inhibitory GABAinterneurons. This ensures efficient local processing and discrete channels of intra-cortical communication. However in the ASD brain, this delicate cytoarchitectonic organisation is disrupted (Casanova et al., 2006), with extensive axonal pathology (Zikopoulos \& Barbas, 2010), abnormal GABA levels (Coghlan et al., 2012) and fewer inhibitory interneurons (Hashemi, Ariza, Rogers, Noctor, \& Martínez-Cerdeño, 2016). Such disruption to the development of cortical circuits is hypothesised to disrupt E:I interactions in ASD, with inhibition unable to curb excessive excitation in the autistic brain (Rubenstein \& Merzenich, 2003; Zikopoulos \& Barbas, 2013), though see Dickinson, Jones, \& Milne (2016). This would manifest as increases in high frequency or 'noisy' activity in the brain (Berg \& Plioplys, 2012; Spence \& Schneider, 2009; Vilidaite, Yu, \& Baker, 2017), and anomalies in high frequency brain activity. This is consistent with findings of increased rates of epilepsy and inter-ictal spikes in ASD (Rubenstein \& Merzenich, 2003). 
Furthermore, disorganisation at the cellular level would have knock-on consequences for the formation of long-distance cortical connections (Khan et al., 2013).

The balance between excitation and inhibition is inherently linked with the concentrations of excitatory neurotransmitters (e.g. glutamate) versus inhibitory neurotransmitters (e.g. GABA) within the brain. This can be measured in-vivo by collecting blood samples and/or using magnetic resonance spectroscopy (MRS), which is an electromagnetic technique designed to non-invasively quantify levels of different chemicals in the brain (Currie et al., 2014). Whilst some studies do show reductions in inhibitory GABA (e.g. Rojas et al., 2014) and increases in excitatory glutamate for autistic samples (e.g. Page et al., 2006), results overall remain equivocal with many studies reporting no ASD-related differences in neurotransmitter concentration (e.g. Horder et al., 2013), reviewed in Dickinson et al., (2016). This could be driven by the small sample sizes employed in MRS research, as well as the inherent spatial inaccuracies of MRS as a technique, discussed further in Currie et al., (2014).

Behavioural approaches (e.g. binocular rivalry and perceptual discrimination thresholds) have also been used to infer ASD-related E-I imbalances. Binocular rivalry is a phenomenon in which visual perception alternates between different stimuli presented separately to each eye. Computational modelling has suggested that greater inhibition vs excitation in the brain can reduce the frequency of perceptual switching between stimuli (Seely \& Crow, 2011). Findings of reduced perceptual switching during binocular rivalry in ASD have therefore been interpreted as evidence for reduced inhibitory processes (Freyberg et al., 2015; Robertson et al., 2013; Robertson et al., 2015). Having said this, Robertson et al., (2015) did not find any differences in occipital GABA concentrations between ASD and control participants, despite slower binocular rivalry rates. Research has also found reduced perceptual thresholds in ASD - i.e. autistic participants can discriminate between closely related visual/auditory stimuli to a greater extent than typically developing controls (Bonnel et al., 2010; Dickinson et al., 2016; Herato et al., 2008; Stanutz et al., 2014). Interestingly, perceptual tasks like orientation and auditory pitch discrimination have been argued to reflect increased rather than decreased inhibition, related to an increased concentration of GABA ( $\mathrm{Li}$ et al., 2008).

Overall, whilst there is good evidence for an E:I imbalance in ASD within cortical microcircuits, the specific direction of the imbalance is currently disputed (Dickinson et al., 2016). To bridge this gap in the literature, multimodal research combining techniques such 
as MRS, psychophysics, computation modelling and neuroimaging M/EEG data, is needed (also see Section 1.4.1).

\subsubsection{Aberrant cortical connectivity in ASD}

At the systems level, the idea of disorganised cortical connectivity has dominated the field, with a number of reviews reporting on structural and functional MRI research (Belmonte et al., 2004; Geschwind \& Levitt, 2007; Müller et al., 2011; Rippon et al., 2007; Vissers, Cohen, \& Geurts, 2012), which typically highlights long-distance structural and functional underconnectivity between cortical regions, resulting in dysfunctional cortical communication and concomitant impacts on a range of perceptual and cognitive processes in ASD (Hughes, 2007; Just, Cherkassky, Keller, Kana, \& Minshew, 2007; Kana, Keller, Minshew, \& Just, 2007; Koshino et al., 2008).

Whilst findings of whole-brain hypoconnectivity in ASD appear reliable (Hughes, 2007; Vissers et al., 2012), the nature of local connectivity in ASD is much more equivocal (Dajani \& Uddin, 2016; Keown et al., 2013; Maximo, Keown, Nair, \& Müller, 2013; Paakki et al., 2010). This may be a consequence of methodology, with measures of local connectivity requiring the temporal resolution appropriate to the transient nature of local processing (Stokes, Wolff, \& Spaak, 2015). Therefore, the research presented in this thesis utilised the excellent spatiotemporal properties of magnetoencephalography, MEG, (Chapters 3-6). Previous studies using EEG and MEG have identified patterns of cortical oscillations which would be consistent with localised hyper-reactivity and an E:I imbalance (Cornew, Roberts, Blaskey, \& Edgar, 2012; Khan et al., 2013; Orekhova et al., 2007).

\section{4 "Oscillopathies" and atypical connectivity in ASD}

The main aim of this thesis is to elucidate the cortical mechanisms underlying low-level sensory and higher-level cognitive differences associated with ASD. One promising neural correlate of these differences, is a dysregulation of neural oscillations - rhythmical changes in neural activity. Neural oscillations are traditionally broken down into canonical frequency bands $(1-3 \mathrm{~Hz}$, delta; $4-7 \mathrm{~Hz}$, theta; $8-12 \mathrm{~Hz}$, alpha; $13-30 \mathrm{~Hz}$, beta; $40-80 \mathrm{~Hz}$, gamma; $>80 \mathrm{~Hz}$, high gamma), each with a specific functional role in neural processing (Buzsáki \& Draguhn, 2004; Lopes da Silva, 2013). There is emerging evidence showing that ASD is characterised by disrupted patterns of cortical oscillations, collectively termed "oscillopathies", reviewed by Kessler et al., (2016) and Simon \& Wallace (2016).

\subsubsection{Gamma-band activity in ASD: visual and auditory processing}


As mentioned in Section 1.3.2, there has been particular interest in characterising patterns of atypical high-frequency gamma-band activity $(>40 \mathrm{~Hz})$ in ASD. Gamma oscillations are generated through the coordinated interactions between excitatory and inhibitory populations of neurons (Buzsáki \& Wang, 2012), which are detectable as local field potentials. Inhibitory parvalbumin-expressing interneurons have been shown to be particularly important for the generation of gamma activity (Bragin et al., 1995). Findings of atypical gamma-band activity for autistic participants would therefore link with the proposed E-I imbalance in ASD (Rubenstein \& Merzenich, 2003; Zikopoulos \& Barbas, 2013).

Gamma-band oscillations play an important role in sensory processing (Buzsáki \& Wang, 2012; Kessler et al., 2016; Simon \& Wallace, 2016; Tallon-Baudry \& Bertrand, 1999; Whittington, Cunningham, LeBeau, Racca, \& Traub, 2011). By synchronising at gamma frequencies, spatially separate neuronal assemblies, each encoding specific aspects of a stimulus, can be bound into a coherent whole (Singer \& Gray, 1995). In this "Binding by Synchrony" account, gamma synchrony determines optimal neuronal response timing (Buzsáki \& Wang, 2012; Whittington et al., 2011) and ensures accuracy in stimulus processing. A distinct but related account ('communication through coherence') suggests that gamma-band synchrony is crucial for the efficient exchange of information between neuronal assemblies at the local and global scales (Fries, 2015). By oscillating at gamma frequencies, neuronal inhibition is confined to a specific part of the gamma cycle, allowing postsynaptic neurons to spike in a temporally synchronised manner (Tiesinga, Fellous, Salinas, José, \& Sejnowski, 2004). Inter-regional communication is achieved through gamma synchronisation between neurons, i.e. when periods of excitability align (communication is "pulsative", Fries, 2015). This is hypothesised to render neural communication precise and effective (Bastos, Vezoli, \& Fries, 2015); not only during sensory processing but across multiple cognitive domains. Alterations to gammaband synchrony would therefore affect perceptual processes, and perhaps cognitive processing more generally (Brock, Brown, Boucher, \& Rippon, 2002). For this reason, gamma oscillations have been proposed as a useful candidate frequency for studying the neural basis of ASD (Brock et al., 2002).

Before reviewing the literature, it is important to expand on the concept of "gamma" activity (Rojas \& Wilson, 2014). Spectral power is made up of 'evoked' activity, phaselocked to stimulus presentation, and 'induced' power not phase-locked to stimulus presentation. Evoked gamma is measured by computing time-frequency responses on trial-averaged data, whereas induced gamma is measured by computing time-frequency 
responses on single trials, and then averaging power across trials* (David, Kilner, \& Friston, 2006). Inter-trial coherence, sometimes called the phase-locking factor, can also be used to measure evoked gamma by quantifying phase consistency over trials. This distinction between evoked/induced gamma is important, because evoked responses occur at early latencies, reflecting bottom-up sensory processes, whereas induced responses are sustained for longer periods of time, reflecting higher-level processes such as perceptual binding (for more information see: Rippon et al., 2007; Rojas \& Wilson, 2014). Finally, measures of coherence, quantifying the consistency of phase relationships, can be used to measure gamma-band connectivity between regions (Bastos \& Schoffelen, 2016).

In the visual domain, there is widespread evidence for atypical gamma in ASD. The power of gamma-band oscillations are generally tied to stimulus properties, for example the spatial frequency of visual gratings (Muthukumaraswamy \& Singh, 2008), or motion intensity (Siegel, Donner, Oostenveld, Fries, \& Engel, 2007). This stimulus-dependent modulation of gamma is reduced (Milne, Scope, Pascalis, Buckley, \& Makeig, 2009; Sysoeva, Galuta, Davletshina, Orekhova, \& Stroganova, 2017) or even absent in participants with ASD (Snijders et al., 2013). Grice et al., (2001), also found that in participants with ASD induced gamma power was not modulated in relation to upright vs inverted faces, suggesting that sensory differences may underlie higher-level social impairments in ASD (also see Wright et al., (2012)). On the other hand, Peiker, Schneider, et al., (2015) reported greater modulation of total gamma power in response to visual motion intensity for ASD participants. M/EEG studies aimed at investigating perceptual binding have also shown atypical patterns of gamma-band activity in ASD. Using illusory Kanisza shapes, one study reported increased induced gamma power (Brown, Gruber, Boucher, Rippon, \& Brock, 2005), whilst another found reduced evoked gamma from 120270ms (Stroganova et al., 2012). Using illusory Mooney faces, Sun et al., (2012) reported reduced gamma-band inter-trial coherence in ASD, alongside reductions in evoked and induced gamma power from 0-400ms post-stimulus onset. Atypical gamma-band connectivity in ASD has also been reported during visual perception. Using MEG, Peiker, David, et al., (2015) quantified patterns of interhemispheric gamma-band coherence as participants identified moving objects presented through a narrow horizontal slit. Their results showed that, compared with controls, ASD participants showed reduced gammaband coherence within the posterior superior temporal sulcus, accompanied by lower

\footnotetext{
* A pure measure of induced power, rather than total power, would also involve the subtraction of evoked responses.
} 
identification accuracy. In contrast, another MEG study using a higher-level visuospatial reasoning task in young children, reported increased patterns of gamma-band coherence between occipital and frontal sensors in ASD (Takesaki et al., 2016).

Findings of atypical gamma have also been reported in the auditory domain. Wilson et al., (2007) for example, used MEG combined with a monaural click train, amplitudemodulated at 40 cycles/second, to elicit an auditory steady-state response (ASSR) of increased $40 \mathrm{~Hz}$ power in the auditory cortex. In contrast to controls, the ASD group showed reduced left hemisphere power with no clear $40 \mathrm{~Hz}$ steady state response. In a follow-up study (Rojas et al., 2011) found reduced ASSR gamma power and inter-trial coherence (ITC: a measure of phase consistency across trials) in first-degree relatives of people with ASD, suggesting that gamma-abnormalities could act as a potential endophenotype for the condition. Reduced gamma-band activity in response to pure auditory tones has also been reported in ASD children (Gandal et al., 2010). Indeed, one large-scale study measured low as well as high frequency responses to pure tones at 200 , 300,500 , and $1,000 \mathrm{~Hz}$ in a sample of 105 ASD children (Edgar et al., 2015). Compared with typically-developing controls, the ASD group showed higher levels of pre-stimulus power in all frequencies, and smaller evoked gamma activity from 50-200ms in response to all stimuli. A longitudinal study of 6-11 year old children using sinusoidal tones found reduced transient gamma ITC around $0.1 \mathrm{~s}$ post-stimulus onset, across developmental time-points (Port et al., 2016). Similar findings of reduced evoked and induced gamma in ASD have been reported for a continuous word recognition task (McFadden, Hepburn, Winterrowd, Schmidt, \& Rojas, 2012).

Across visual and auditory domains, the literature reviewed in this section suggests that local gamma-band activity within primary and associative sensory cortices are affected in ASD (Kessler et al., 2016; Simon \& Wallace, 2016). Findings across such studies are complex to unite but, overall, they support an ASD neuro-oscillatory phenotype of reduced evoked gamma during the early sensory processing, combined with failure of later evoked and induced gamma power regulation as a function of stimulus characteristics (e.g. spatial frequency of visual gratings, perceptual binding for illusory shapes, and neural entrainment for auditory clicktrains). Furthermore, findings of reduced gamma-band synchrony and connectivity in ASD, imply that the phase of gamma oscillations are less consistently organised over time, which would affect cortical communication during sensory processing (Fries, 2015; Peiker, David, et al., 2015). We therefore propose a shift away from a singular view of gamma-band dysfunction in ASD. Instead, the evidence is more consistent with the view that gamma oscillations in ASD are dysregulated, possibly 
due to a suboptimal balance between excitatory and inhibitory populations of neurons (Snijders et al., 2013; Zikopoulos \& Barbas, 2013), see Section 1.5.1. This could hypothetically result in both increases or decreases to gamma-power and synchrony depending on the specific task and/or symptom profile of the ASD cohort (Kessler et al., 2016).

\subsubsection{Task-related cross-frequency, phase-amplitude coupling (PAC) in ASD}

One oscillatory mechanism associated with the regulation of gamma-band activity is phase-amplitude coupling (PAC) - a form of cross-frequency coupling in which the phase of a low-frequency oscillation is coupled to the amplitude of a high-frequency oscillation, usually gamma (Canolty et al., 2006; Canolty \& Knight, 2010; Jensen \& Colgin, 2007). PAC has been proposed to act as a mechanism for the dynamic coordination of brain activity over multiple spatial scales, with the amplitude of high-frequency gamma-band activity within local ensembles coupled to large-scale patterns of low-frequency phase synchrony (Bonnefond, Kastner, \& Jensen, 2017). In this way, it is hypothesised that topdown mechanisms, such as attention, can influence gamma activity within local ensembles (Bonnefond \& Jensen, 2015; Park et al., 2016). Increases in alpha-gamma PAC are frequently reported during visual processing (Voytek et al., 2010), potentially via inter-laminar coupling within visual cortex (Mejias, Murray, Kennedy, \& Wang, 2016; Spaak, Bonnefond, Maier, Leopold, \& Jensen, 2012), see Figure 1.1. The neurophysiological generation of PAC is thought to be closely tied to the balance between excitatory and inhibitory (E-I) populations of neurons (Mejias et al., 2016; Onslow, Jones, \& Bogacz, 2014). If this delicate E-I balance is affected in ASD, as argued by Rubenstein \& Merzenich, (2003) and Zikopoulos \& Barbas (2013), it would be hypothesised that neural activity in ASD would be associated with reductions in PAC. 


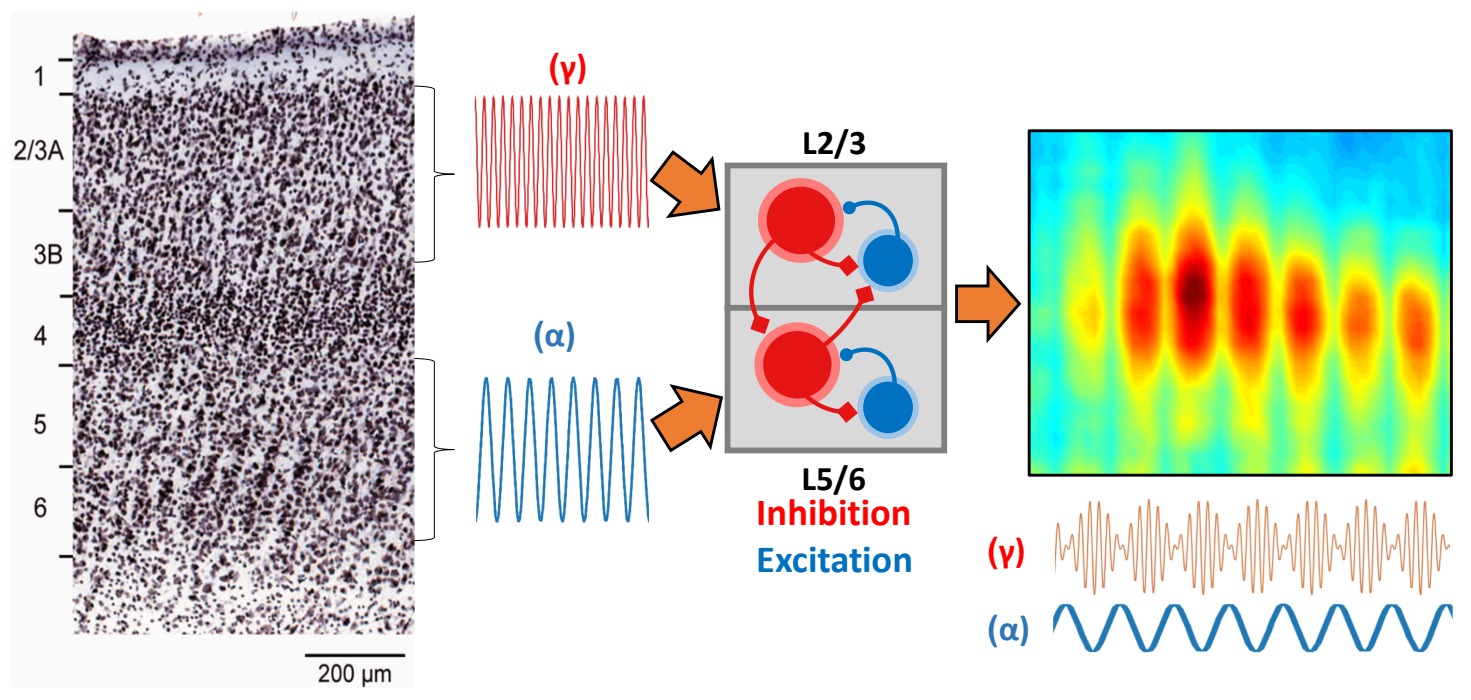

Figure 1.1 Illustration of mechanisms of phase-amplitude coupling (PAC). Gamma (30-80 $\mathrm{Hz}$ ) and alpha (8-13 Hz) oscillations emerge separately from supragranular layers (2/3) and superficial layers (5/6) of cortex. These rhythms are thought to interact via an interlaminar coupling circuit based on interactions between excitation (blue) and inhibition (red) (Mejias et al., 2016; Spaak et al., 2012). This results in the amplitude/power of the supragranular gamma rhythm becoming entrained to the phase of the superficial alpha. The photomicrograph of cortical layers has been reproduced from (Markov et al., 2014).

One previous MEG study found reduced alpha-gamma PAC in the fusiform face area during emotional face processing in a group of autistic adolescents (Khan et al., 2013). Importantly, differences in PAC were present despite equivalent evoked potentials and alpha/gamma power between ASD and control groups. In addition, Khan et al., (2013) reported that local PAC was related to patterns of long-range alpha hypoconnectivity in autism, suggesting that local and long-range connectivity are concurrently affected. A recent study using the same stimuli, however, found no PAC differences in a group of autistic children, versus typically developing controls (Mamashli et al., 2018). Alterations in the developmental trajectories of PAC could therefore be specific to late childhood and adolescence. However longitudinal studies of PAC would be needed to test this idea. One other study has examined PAC in ASD, reporting abnormal patterns of alpha-gamma PAC in ASD using resting-state MEG data, (Berman et al., 2015).

Based on these studies, and findings more generally of local dysregulation in ASD (see Section 1.5.1), we hypothesise that for ASD participants the amplitude of gamma-band oscillations generated during sensory processing will be less coupled to the phase of 
lower-frequency oscillations (i.e. reduced PAC). We tested this hypothesis in Chapter 3, using a paradigm with an embedded visual grating.

\subsubsection{Global oscillatory connectivity deficits in ASD}

Whilst high-frequency oscillatory activity is a good proxy for local processing, results from the fMRI literature demonstrate that global brain connectivity is reduced in ASD (Hughes, 2007; Just et al., 2007; Rippon et al., 2007). Neurophysiologically, patterns of connectivity mediated via low frequency oscillations represent a promising research avenue for the study of long-distance brain connectivity (Varela, Lachaux, Rodriguez, \& Martinerie, 2001; Wang, Saalmann, Pinsk, Arcaro, \& Kastner, 2012). A small but growing number of M/EEG research papers have, indeed, shown reduced low-frequency oscillatory activity in ASD individuals, during set-shifting, slit-viewing, emotional face processing and number estimation tasks (Bangel et al., 2014; Doesburg, Vidal, \& Taylor, 2013; Khan et al., 2013; Leung, Ye, Wong, Taylor, \& Doesburg, 2014; Peiker, David, et al., 2015).

Application of functional connectivity measures to 'resting-state' or 'task-free' M/EEG data has also shown reductions in global connectivity for the autistic brain (Barttfeld et al., 2012; Pollonini et al., 2010). For example, Kitzbichler et al., (2015) collected resting-MEG data from 15 ASD / 15 control participants, aged 6-21, and applied various graph theory metrics to quantify network organisation. In the gamma band $(30-70 \mathrm{~Hz})$, the ASD group showed stronger and more efficiently connected networks, with a greater number of connections from occipital areas to parietal, temporal and frontal regions. In the beta band (13-30Hz), the ASD group had less efficiently connected networks, particularly those involving the frontal/parietal lobes. Overall, the authors interpret these differences as an imbalance between feedforward mechanisms, primarily mediated by gamma, and frontoparietal regulatory feedback mechanisms, mediated by lower-frequency oscillations.

In order to expand on these ideas, it is important for the field to establish the direction of global connectivity differences for autistic samples. Functional connectivity mediated by low-frequency oscillations is widely acknowledged to underlie cognitive control (theta) (Cavanagh \& Frank, 2014), functional inhibition (alpha) (Klimesch, 2012) and top-down processes (beta) (Engel \& Fries, 2010). Disruptions to global patterns of low-frequency oscillatory connectivity in ASD may therefore reflect reduced feedback connectivity, through which higher-level fronto-parietal regions constrain and shape processing for lower-level sensory cortices (Bonnefond \& Jensen, 2015; Kessler et al., 2016; Kitzbichler et al., 2015). Interestingly, a recent study showed that during somatosensory stimulation, feedforward connectivity from primary to secondary somatosensory cortex is increased in 
ASD (Khan et al., 2015). Feedforward pathways in the autistic brain may therefore overcompensate for the lack of feedback connectivity. However, few M/EEG studies have specifically quantified patterns of feedforward versus feedback oscillatory connectivity in ASD. Therefore, in this thesis (Chapters $3,5,6$ ), we address this gap by using a frequency-resolved measure of directed connectivity called non-parametric Granger Causality (see Chapter 3 for more details).

\subsection{A Novel approach to ASD: local dysregulation, global hypoconnectivity, and deficient predictive coding}

\subsubsection{Local Dysregulation}

The rather heterogeneous findings reviewed in the previous section demonstrate that potential 'oscillopathies' in ASD are complex. Gamma activity has been found to be both increased and decreased in terms of power (evoked/induced) and connectivity (Peiker, David, et al., 2015; Simon \& Wallace, 2016; Takesaki et al., 2016). We propose a novel approach which shifts the emphasis away from hyper/hypo-connectivity as reflected by gamma power and phase-synchrony alone (Picci, Gotts, \& Scherf, 2016). Instead, local patterns of neural activity in ASD can be described as "dysregulated" (Kessler et al., 2016), potentially reflecting an imbalance between excitatory and inhibitory neural populations (Rubenstein \& Merzenich, 2003). This "dysregulation" is reflected by findings of: gamma activity inappropriately modulated by the properties of sensory stimuli (Milne et al., 2009; Peiker, Schneider, et al., 2015); as well as reduced phase amplitude coupling (Berman et al., 2015; Khan et al., 2013). Where local activity is affected, this would result in suboptimal signal-to-noise ratios during sensory processing in ASD (Rubenstein \& Merzenich, 2003; Van de Cruys, Van der Hallen, \& Wagemans, 2017), especially in situations with multiple competing stimuli and where perceptual binding is required (Brock et al., 2002). Additionally, local dysregulation could result in excessively strong or weak patterns of neural activity for certain stimuli, potentially underlying the hyper and hyposensitive responses reported by autistic people (Leekam et al., 2007). This proposal links with the idea of "hyper-functional microcircuits", outlined in the 'intense world' theory of ASD (Markram \& Markram, 2010). In sum, we suggest that local connectivity in ASD is dysregulated, which is more appropriately quantified through measures of neural excitation-inhibition and cross-frequency coupling, rather than gamma-power and phasesynchrony alone (Berman et al., 2015; Kessler et al., 2016; Simon \& Wallace, 2016).

Given the increasing interest in characterising patterns of cross-frequency coupling in ASD (Berman et al., 2015), it is vital for the field to use robust and reliable measures of 
PAC, combined with an appreciation of the methodological pitfalls (Aru et al., 2015; Dvorak \& Fenton, 2014). Therefore, in Chapter 2, we present research investigating various methods for quantifying PAC, alongside an openly available MATLAB pipeline. In Chapter 3 we apply this pipeline to MEG data collected from adolescent participants diagnosed with ASD and age-matched controls, in order to characterise differences in alpha-gamma PAC during visual processing and test the hypothesis of dysregulated PAC in ASD.

In Chapter 4, we investigated local gamma-band dysregulation in the auditory domain, using clicktrain stimuli to elicit bilateral $40 \mathrm{~Hz}$ auditory steady state responses (ASSRs). As outlined in section 1.4.1, previous research has found reductions in ASSRs in autistic participants and the first-person relatives of people with ASD (Rojas et al., 2011; Wilson et al., 2007). Using MEG, we attempted to replicate and extend these findings using updated analysis methods.

\subsubsection{Global Hypoconnnectivity}

In addition to local dysregulation, the literature reviewed in section 1.4 .3 suggests that ASD is associated with reduced patterns of global functional connectivity. These reductions in connectivity are related to processes of phase-synchrony, mediated by oscillations in the theta, alpha and beta-bands (Bangel et al., 2014; Doesburg et al., 2013; Khan et al., 2013; Leung et al., 2014). Furthermore, recent work suggests that feedforward pathways may be overexpressed in the ASD brain (Khan et al., 2015), relative to feedback pathways mediated by low-frequency oscillations (Khan et al., 2013; Kitzbichler et al., 2015). In Chapter 3 we specifically investigate feedback vs feedforward connectivity in ASD during visual processing. Using MEG, Georgios Michalareas and colleagues recently showed that the human visual system operates via distinct oscillatory communication channels, with gamma oscillations mediating feedforward connectivity and alpha oscillations mediating feedback connectivity (Michalareas et al., 2016). These MEG findings are built upon substantial research in macaques, using electrocorticography and invasive neural stimulation, which has firmly established frequency-specific feedforward/feedback connectivity in the primate visual system (Bastos et al., 2015; Kerkoerle et al., 2014). Using a similar analysis pipeline to Michalareas et al., (2016), we hypothesised that in ASD, connectivity within the visual system would be characterised by reduced alpha feedback connectivity, but potentially increased gamma feedforward connectivity (Kessler et al., 2016). 


\subsubsection{Local vs Global Connectivity in ASD}

One previous MEG study reported that local and global connectivity are concurrently affected in ASD (Khan et al., 2013). Where local activity is dysregulated, this would theoretically disrupt the establishment of long-range connectivity, due to suboptimal signal-to-noise ratios (Rubenstein \& Merzenich, 2003). However the reverse could also be true: reduced patterns of global connectivity in ASD could result in dysregulated local gamma activity, unconstrained by low-frequency phase-synchrony underlying top-down, cognitive control mechanisms (Bonnefond \& Jensen, 2015; Khan et al., 2013; Rippon et al., 2007). In Chapter 3 of this thesis, the association between local and global connectivity in ASD was investigated, by correlating patterns of local alpha-gamma PAC with inter-regional alpha-band feedback connectivity within the same participants.

\subsubsection{Deficient Predictive Coding?}

The combined effect of local dysregulation and global hypoconnectivity would be consistent with predictive-coding theories of ASD (Lawson et al., 2014; Palmer et al., 2017; Pellicano \& Burr, 2012). In the typically developing brain, efficient Bayesian inference is dependent upon feedback pathways carrying top-down predictions down the cortical hierarchy (Friston, 2005, 2008). There is emerging evidence that low-frequency alpha/beta oscillations are crucial for this process (Auksztulewicz, Friston, \& Nobre, 2017; Chao, Takaura, Wang, Fujii, \& Dehaene, 2018; Giraud \& Arnal, 2018), allowing the brain to predict "what" should happen and "when" this should occur (Arnal \& Giraud, 2012). In the case of ASD, where global patterns of feedback connectivity are affected, this would impact the brain's ability to predict upcoming events. Sensory processing in the brain would therefore be forced from a predictive to a reactive process, with excessive prediction error signalling and increased feedforward connectivity (Kessler et al., 2016; Khan et al., 2015). This proposal is therefore theoretically consistent with the "hypo-prior" account of Pellicano \& Burr (2012). In contrast to the notion of hypo-priors, hyperprecision accounts argue that ASD symptoms emerge due to a failure to correctly weight predictions in a context-dependent manner (Lawson et al., 2014; Palmer et al., 2017). Again, this would be consistent with increasingly dysregulated local and global connectivity in ASD for complex, noisy environments, and during processing of contextdependent stimuli, such as social cues (Lawson et al., 2017). The proposed connectivity framework could therefore help unifying different versions of the predictive coding hypothesis of ASD.

In Chapter 5, we investigate predictive-coding theories of ASD, using a robust auditory oddball paradigm to compare evoked mismatch fields to unexpected deviant stimuli and 
unexpected silences (Recasens \& Uhlhaas, 2017). In addition we attempted to differentiate between "hypo-prior" and "hyper-precision" accounts by presenting the tones in silence as well as babble noise (Dreschler, Verschuure, Ludvigsen, \& Westermann, 2001).

\subsubsection{Extending the framework beyond sensory aberrations}

Whilst the proposed framework has primarily considered low-level sensory findings in ASD, similar patterns of local dysregulation and reduced global connectivity could also underlie higher-level social and cognitive impairments in ASD. As mentioned in section 1.2.2, social stimuli are generally context-dependent, requiring more complex top-down predictions than standard non-social objects (Otten, Seth, \& Pinto, 2017; Palmer et al., 2015). This is reflected by the complex network of brain regions involved in social cognition, including the medial pre-frontal cortex, orbitofrontal cortex, amygdala, superior temporal sulcus, fusiform gyrus and posterior cingulate cortex (Alcalá-López et al., 2017; Padmanabhan, Lynch, Schaer, \& Menon, 2017). Connectivity between these regions increases throughout childhood and adolescence, as social processing becomes increasingly refined (Blakemore, 2012). Additionally, recent work utilising MEG and TMS has shown that theta-band $(4-8 \mathrm{~Hz})$ oscillations, play a crucial role in the formation of cortical networks underlying high-level perspective taking and mentalizing (Bögels, Barr, Garrod, \& Kessler, 2015; Gooding-Williams, Wang, \& Kessler, 2017; Wang, Callaghan, Gooding-Williams, McAllister, \& Kessler, 2016) . In the case of ASD, we hypothesise that social impairments would be caused by alterations to local and global connectivity, reflecting atypical developmental trajectories in the maturation of social brain networks (McPartland \& Jeste, 2015). In support of this, fMRI studies have shown reduced global connectivity between regions of the social brain network in ASD (Gotts et al., 2012; von dem Hagen, Stoyanova, Baron-Cohen, \& Calder, 2012). Interestingly, using diffusion tensor imaging, one study recently showed reductions in local structural connectivity for ASD, which were associated with measures of social dysfunction (d'Albis et al., 2018). However, to our knowledge, no previous MEG study including ASD participants has examined patterns of theta-band phase synchrony during high-level social/cognitive processing. In Chapter 6, we therefore investigated the oscillation-based connectivity during embodied perspective-taking in a group of adult controls aged 23-45 (Experiment 1 ); as well as a group of adolescents diagnosed with ASD and age-matched controls aged 14-20 (Experiment 2). 


\subsection{Thesis Aims}

The primary aim of research presented in this thesis, was to investigate atypical cortical activity in ASD, using MEG. I focus on characterising potential "oscillopathies", i.e. atypical patterns of neural oscillations, in terms of power, phase synchrony and phase amplitude coupling. The secondary aim was to develop robust analysis pipelines for measuring oscillation-based connectivity, especially in relation to measures of phase-amplitude coupling (see Chapter 2).

Based on the previous EEG/MEG literature, reviewed in Section 1.4, we propose a novel framework for the study of oscillopathies in ASD, see Section 1.5, based on local dysregulation and global hypoconnectivity. This framework is able to accommodate heterogeneous research findings (Brown, Gruber, Boucher, Rippon, \& Brock, 2005; Gandal et al., 2010; Grice et al., 2001; Kessler et al., 2016; Milne et al., 2009; Simon \& Wallace, 2016; Snijders et al., 2013; Sun et al., 2012; Takesaki et al., 2016; Wright et al., 2012), especially in relation to gamma activity, linking with E-I theories at the cellular level, as well as neurocognitive, predictive-coding theories of ASD.

To test these ideas, MEG data were acquired using an Elekta Neuromag scanner (Aston University, Chapters 2,3,4,6) and a KIT-Macquarie MEG160 scanner (Macquarie University, Chapter 5). Please refer to Table 1.1 for more details. Data were analysed using the Fieldtrip toolbox (Oostenveld, Fries, Maris, \& Schoffelen, 2011), and source analysis was performed using beamforming (Hillebrand \& Barnes, 2005). In Chapters 2-5 we focussed on low-level sensory processing in ASD (visual and auditory), whereas in Chapter 6 we investigated the neural basis of higher-level embodied perspective-taking in ASD. 


\begin{tabular}{|c|c|c|c|c|c|c|c|c|}
\hline $\begin{array}{c}\text { Dataset } \\
\text { Reference }\end{array}$ & $\begin{array}{c}\text { MEG } \\
\text { Scanner }\end{array}$ & Paradigms & $\begin{array}{c}\text { Number of Participants } \\
\text { (Analysed Data) }\end{array}$ & $\begin{array}{c}\text { Number of } \\
\text { Participants } \\
\text { (Rejected } \\
\text { Data) }\end{array}$ & Participant Age & Raven Matrices /60 & $\begin{array}{l}\text { Autistic Quotient } \\
\qquad / 50\end{array}$ & Chapter \\
\hline A & $\begin{array}{c}\text { Elekta } \\
\text { Neuromag }\end{array}$ & Visual Grating & 16 Adults ( 6 male; 10 female) & 0 & $\begin{array}{l}\text { Mean }=28.25 ; \mathrm{SD}^{* *}= \\
6.23 ; \text { Range }=19-45\end{array}$ & Not collected & Not Collected & 2 \\
\hline B & $\begin{array}{c}\text { Elekta } \\
\text { Neuromag }\end{array}$ & $\begin{array}{l}\text { Visual Grating, } \\
\text { Auditory } \\
\text { Clicktrain, } \\
\text { Perspective- } \\
\text { Taking }\end{array}$ & $\begin{array}{l}18 \text { ASD (14 male; } 4 \text { female) } \\
18^{*} \text { Age-Matched Controls (15 } \\
\text { male; } 3 \text { female) }\end{array}$ & $\begin{array}{l}5 \text { ASD } \\
4 \text { Control }\end{array}$ & $\begin{array}{c}\text { Mean }=16.67 ; \mathrm{SD}= \\
3.2 ; \text { Range }=14-20 \\
\text { Mean = 16.89; SD = } \\
2.8 ; \text { Range }=14-20\end{array}$ & $\begin{array}{c}\text { Mean }=43.8 ; \\
\text { SD }=7.9 ; \\
\text { Range }=22-56 \\
\text { Mean }=48.7 \\
\text { SD }=5.7 \\
\text { Range }=37-56\end{array}$ & $\begin{array}{c}\text { Mean }=32.6 ; \\
\mathrm{SD}=6.6 ; \\
\text { Range }=21-46 \\
\text { Mean }=10.9 ; \\
\mathrm{SD}=5.6 ; \\
\text { Range }=6-21\end{array}$ & $3,4,6$ \\
\hline C & $\begin{array}{c}\text { KIT- } \\
\text { Macquarie } \\
\text { MEG160 }\end{array}$ & Auditory Oddball & $\begin{array}{l}16 \text { Age-Matched Controls (13 } \\
\text { male; } 3 \text { female) }\end{array}$ & 4 ASD & $\begin{array}{l}\text { Mean = 15.23; SD = } \\
\text { 3.10; Range = 10-19 }\end{array}$ & $\begin{array}{c}\text { Mean }=44.2 ; \\
\text { SD }=7.8 ; \\
\text { Range }=28-59 \\
\text { Mean }=47.6 ; \\
\text { SD }=8.1 ; \\
\text { Range }=32-58\end{array}$ & $\begin{array}{c}\text { Mean }=35.8 \\
S D=7.1 \\
\text { Range }=25-47\end{array}$ & 5 \\
\hline $\mathrm{D}$ & $\begin{array}{c}\text { Elekta } \\
\text { Neuromag }\end{array}$ & $\begin{array}{l}\text { Perspective- } \\
\text { Taking }\end{array}$ & 18 adults ( 4 male; 14 female) & 0 & $\begin{array}{c}\text { Mean = 27.55; SD = } \\
\text { 5.86; Age-Range = } \\
23-45\end{array}$ & Not Collected & Not Collected & 6 \\
\hline
\end{tabular}

* Please note, only 17 age-matched controls completed the perspective-taking task in dataset B (see Chapter 6); ** SD = Standard Deviation Table 1.1 Details of the MEG data presented in this thesis. 


\subsection{Participant Information}

For dataset $A$ and $D$ (see Table 1.1) data were collected from adult participants (aged 1945) recruited from the Aston Brain Centre and Aston University Psychology Participant Pool. Participants reported no history of neurological or psychiatric illness. A subset of participants were compensated $£ 20$ for their time.

For dataset $B$ (see Table 1.1) data were collected from 18 adolescent participants diagnosed with ASD and 18 typically developing controls (TDCs). In addition, data were collected from a further 9 participants, but excluded from analysis due to: intolerance to MEG resulting in incomplete data collection (2 ASD); movement over $0.5 \mathrm{~cm}$ (2 ASD, 2 control; threshold defined before data collection); metal on participant resulting in artefactual MEG data (1 ASD, 1 control); AQ score over 30 (1 control; threshold defined before data collection). Data from the three separate tasks were collected in one scan session (always in same order: resting-state ${ }^{*}$, grating/clicktrain, perspective-taking). To avoid fatigue, participants took frequent breaks and the total scan time was minimised to 45-50 minutes. All ASD participants had a confirmed clinical diagnosis of "Autism Spectrum Disorder" or "Asperger's Syndrome" (i.e. participants met the diagnostic criteria as outlined in the DSM 4 or DSM 5) as assessed by a qualified paediatric psychiatrist. Evidence for this was provided through a copy of a doctors letter. Control participants were excluded if a sibling or parent was diagnosed with ASD. Participants taking psychiatric medication or diagnosed with another psychiatric condition (defined by DSMV) were excluded from participating. ASD participants were recruited through local schools, social media support groups and adolescent ASD advocacy groups. Typically developing controls were recruited through local schools. Participants were compensated $£ 20$ for their time. Basic cognitive functioning was measured using the Raven’s Progressive Matrices (Raven, 1936; see Table 1.1). No participant could be classed as having an intellectual disability. Autistic traits were measured using the Autism Quotient questionnaire (see Table 1.1). Whilst 6 participants scored under the arbitrary threshold of 25 on the $A Q$, this questionnaire is not clinically diagnostic and therefore these participants were included in the analysis.

For dataset $C$ (see Table 1.1), data were collected from 16 adolescent participants diagnosed with ASD and 16 typically developing controls (TDCs) using the same inclusion/exclusion criteria as for dataset $B$. Data from a further 4 ASD participants were collected but excluded from analysis due to intolerance to MEG resulting in incomplete

\footnotetext{
${ }^{*}$ Not analysed for this thesis
} 
data collection resulting in movement over $0.5 \mathrm{~cm}$ ( $3 \mathrm{ASD}$; threshold defined before data collection) and a technical issue with marker-coil measurement (1 ASD). ASD participants were recruited through local schools, social media support groups and adolescent ASD advocacy groups. Typically developing controls were recruited through local schools and the Macquarie "Neuronauts" participant pool. Participants were compensated $\$ 50$ for their time.

\subsection{Thesis Outline}

The thesis is organised as follows. In Chapter 2, using pilot MEG data, I investigated various issues and ambiguities in characterising alpha-gamma PAC. Additionally, a robust and openly-available analysis pipeline was established, to characterise changes in PAC during visual processing. Using this pipeline, Chapter 3 compared alpha-gamma PAC between a group of adolescent ASD participants and age-matched controls. Additionally, inter-regional patterns of directed functional connectivity were estimated between visual areas V1 and V4. Chapter 4 investigated local gamma-band entrainment in ASD, using an auditory clicktrain to generate a steady-state response. Patterns of evoked gamma power and phase synchrony were compared with sustained steady state responses at $40 \mathrm{~Hz}$. Chapter 5 tested predictive-coding theories of ASD, using an auditory oddball paradigm, to evoke mismatch field responses. Chapter 6 investigated the oscillatory connectivity underlying embodied perspective-taking, in ASD and control participants. Finally, in Chapter 7, the main findings of my research are discussed, alongside various limitations and implications for future research. 


\title{
Chapter 2: The Detection of Phase Amplitude Coupling During Sensory Processing
}

\author{
Seymour, R.A ${ }^{1,2,3}$., Rippon, $\mathbf{G}^{1}$., \& Kessler, $\mathbf{K}^{1}$. \\ ${ }^{1}$ Aston Brain Centre, School of Life and Health Sciences, Aston University, Birmingham, \\ B4 7ET. \\ ${ }^{2}$ Department of Cognitive Science, Macquarie University, Sydney, Australia, 2109. \\ ${ }^{3}$ ARC Centre of Excellence in Cognition and Its Disorders, Macquarie University, Sydney, \\ Australia, 2109.
}

Please note, a version of this chapter has been published in a peer-reviewed journal:

Seymour, R. A., Rippon, G., \& Kessler, K. (2017). The Detection of Phase Amplitude Coupling during Sensory Processing. Frontiers in neuroscience, 11, 487.

\subsection{Abstract}

There is increasing interest in understanding how the phase and amplitude of distinct neural oscillations might interact to support dynamic communication within the brain. In particular, previous work has demonstrated a coupling between the phase of low frequency oscillations and the amplitude (or power) of high frequency oscillations during certain tasks, termed phase amplitude coupling (PAC). For instance, during visual processing in humans, PAC has been reliably observed between ongoing alpha $(8-13 \mathrm{~Hz})$ and gamma-band $(>40 \mathrm{~Hz})$ activity. However, the application of PAC metrics to electrophysiological data can be challenging due to numerous methodological issues and lack of coherent approaches within the field. Therefore, in this chapter we outline the various analysis steps involved in detecting PAC, using an openly available MEG dataset from 16 participants performing an interactive visual task. Firstly, we localised gamma and alpha-band power using the Fieldtrip toolbox, and extracted time courses from area V1, defined using a multimodal parcellation scheme. These $\mathrm{V} 1$ responses were analysed for changes in alpha-gamma PAC, using four common algorithms. Results showed an 
increase in alpha $(7-13 \mathrm{~Hz})$ - gamma $(40-100 \mathrm{~Hz})$ PAC in response to the visual grating stimulus, though specific patterns of coupling were somewhat dependent upon the algorithm employed. Additionally, post-hoc analyses showed that these results were not driven by the presence of non-sinusoidal oscillations, and that trial length was sufficient to obtain reliable PAC estimates. Finally, throughout the chapter, methodological issues and practical guidelines for ongoing PAC research will be discussed.

\subsection{Introduction}

Electrophysiological brain oscillations are often separated into distinct frequency bands, ranging from low-frequency delta $(1-4 \mathrm{~Hz})$ to high-frequency gamma $(<40 \mathrm{~Hz})$. The power and/or connectivity profiles of these frequency bands have been linked with specific neuronal and cognitive functions (Buzsáki \& Draguhn, 2004; Palva, Palva, \& Kaila, 2005). Whilst this has proven a powerful tool in neuroscientific research, there is emerging evidence that oscillations from different frequency bands also display specific coupling patterns - a phenomenon termed cross frequency coupling, CFC, (Hyafil, Giraud, Fontolan, \& Gutkin, 2015; Jensen \& Colgin, 2007). One of the best studied forms of CFC is phase-amplitude coupling (PAC), in which the amplitude/power of a high frequency oscillation, often gamma $(>40 \mathrm{~Hz})$, is coupled to the phase of a lower frequency oscillation (Canolty et al., 2006; Canolty \& Knight, 2010). PAC has been observed in multiple regions of the human brain, including the visual cortex (Voytek et al., 2010), auditory cortex (Cho et al., 2015), hippocampus (Heusser, Poeppel, Ezzyat, \& Davachi, 2016; Lega, Burke, Jacobs, \& Kahana, 2014) and prefrontal cortex (Voloh, Valiante, Everling, \& Womelsdorf, 2015; Voytek et al., 2015), in both electrocorticography (ECOG) and magnetoencephalography (MEG) recordings.

Within the visual system, there is strong evidence for a dynamic coupling between alpha phase $(8-13 \mathrm{~Hz}$ ) and gamma amplitude, $>40 \mathrm{~Hz}$, (Bonnefond \& Jensen, 2015; Spaak, Bonnefond, Maier, Leopold, \& Jensen, 2012; Voytek et al., 2010). Alpha oscillations are associated with pulses of cortical inhibition every $100 \mathrm{~ms}$ (Jensen \& Mazaheri, 2010; Klimesch, 2012), whilst supporting communication through phase dynamics (Fries, 2015). In contrast, gamma oscillations emerge through local excitatory and inhibitory interactions, and synchronise local patterns of cortical activity (Buzsáki \& Wang, 2012; Singer \& Gray, 1995). In visual cortex, ongoing gamma-band activity becomes temporally segmented by distinct phases of alpha-band activity (Bonnefond, Kastner, \& Jensen, 2017; Spaak et al., 2012), possibly via inter-laminar coupling between supragranular and infragranular cortical layers (Mejias, Murray, Kennedy, \& Wang, 2016). This coupling has been proposed to act as a mechanism for the dynamic co-ordination of brain activity over multiple spatial scales, 
with high-frequency activity within local ensembles coupled to large-scale patterns of lowfrequency phase synchrony (Bonnefond et al., 2017), both within the visual system (Bonnefond \& Jensen, 2015), and more widespread neurocognitive networks (Florin \& Baillet, 2015). This would allow information to be routed efficiently between areas and for neuronal representations to be segmented and maintained, for example during visual working memory (Bonnefond \& Jensen, 2015; Lisman \& Idiart, 1995). Atypical patterns of PAC have also been proposed to underlie atypical cortical connectivity in several neurological conditions, including autism spectrum disorder (Kessler, Seymour, \& Rippon, 2016; Khan et al., 2013), schizophrenia (Kirihara, Rissling, Swerdlow, Braff, \& Light, 2012) and Parkinson's Disease (De Hemptinne et al., 2013; Özkurt \& Schnitzler, 2011).

Given the developing interest in cross-frequency coupling, it is vital for the wider neuroscience and electrophysiological community to understand the steps involved in its measurement and interpretation. This is especially important for PAC, which is beset with methodological pitfalls, since there are many competing algorithms, approaches, and currently no gold-standard set of analysis steps (Canolty \& Knight, 2010; Jensen, Spaak, $\&$ Park, 2016). It has also been suggested that numerous incidences of reported PAC may in fact be false positives, caused by suboptimal analysis practices and/or the presence of artefacts within the data (Aru et al., 2015; Hyafil, 2015). For example non-sinusoidal sawtooth-like oscillations can generate artificially inflated PAC values, via low-frequency phase harmonics (Cole et al., 2017; Lozano-Soldevilla, ter Huurne, \& Oostenveld, 2016; Vaz, Yaffe, Wittig Jr, Inati, \& Zaghloul, 2017).

In this chapter, we outline a general approach for detecting changes in phase-amplitude coupling during visual processing, using a novel MEG dataset, analysed using the Fieldtrip toolbox (Oostenveld, Fries, Maris, \& Schoffelen, 2010), and openly available MATLAB scripts. Four common PAC algorithms were used to quantify the coupling between ongoing alpha phase $(7-13 \mathrm{~Hz})$ and gamma amplitude/power $(>40 \mathrm{~Hz})$ whilst participants viewed a static visual grating. Given the controversy surrounding PAC analysis, methodological steps were outlined in detail and justified by existing empirical research. Furthermore, follow-up analyses were conducted to establish the reliability of our results and to assess whether patterns of alpha-gamma PAC were driven by nonsinusoidal oscillations or insufficient data. 


\subsection{Materials and Methods}

\subsubsection{Participants}

Data were collected from 16 participants (6 male, 10 female, mean age $=28.25, S D=$ 6.23). All participants had normal or corrected to normal vision and no history of neurological or psychiatric illness.

\subsubsection{Experimental Procedures}

All experimental procedures complied with the Declaration of Helsinki and were approved by the Aston University, Department of Life \& Health Sciences ethics committee.

Participants gave written informed consent before participating in the study.

\subsubsection{Paradigm}

Participants performed an engaging sensory paradigm, Figure 2.1, designed to elicit patterns of high-frequency oscillatory activity. Each trial started with a variable fixation period of $1.5,2.5$ or 3.5 s randomised across trials, followed by the presentation of a visual grating or auditory binaural click train stimulus; however only the visual data will be analysed in this chapter. The visual grating stimulus had a spatial frequency of 2 cycles/degree and was presented for $1.5 \mathrm{~s}$. To keep participants engaged with the task, cartoon pictures of aliens or astronauts were presented after the visual grating, for a maximum of $0.5 \mathrm{~s}$. Please note that visual responses to the alien or astronaut picture did not form part of the MEG analysis. Participants were instructed to respond to the appearance of an alien picture using a response pad (maximum response period of $1.5 \mathrm{~s}$ ). The accuracy of the response was conveyed through audio-visual feedback, followed by a 0.5s fixation period. In total, the MEG recording lasted 12-13 minutes and included 64 trials with visual grating stimuli. Prior to MEG acquisition, the nature of the task was fully explained to participants and several practice trials were performed. Accuracy rates were above $95 \%$ for all participants indicating that the task was engaging and successfully understood. 


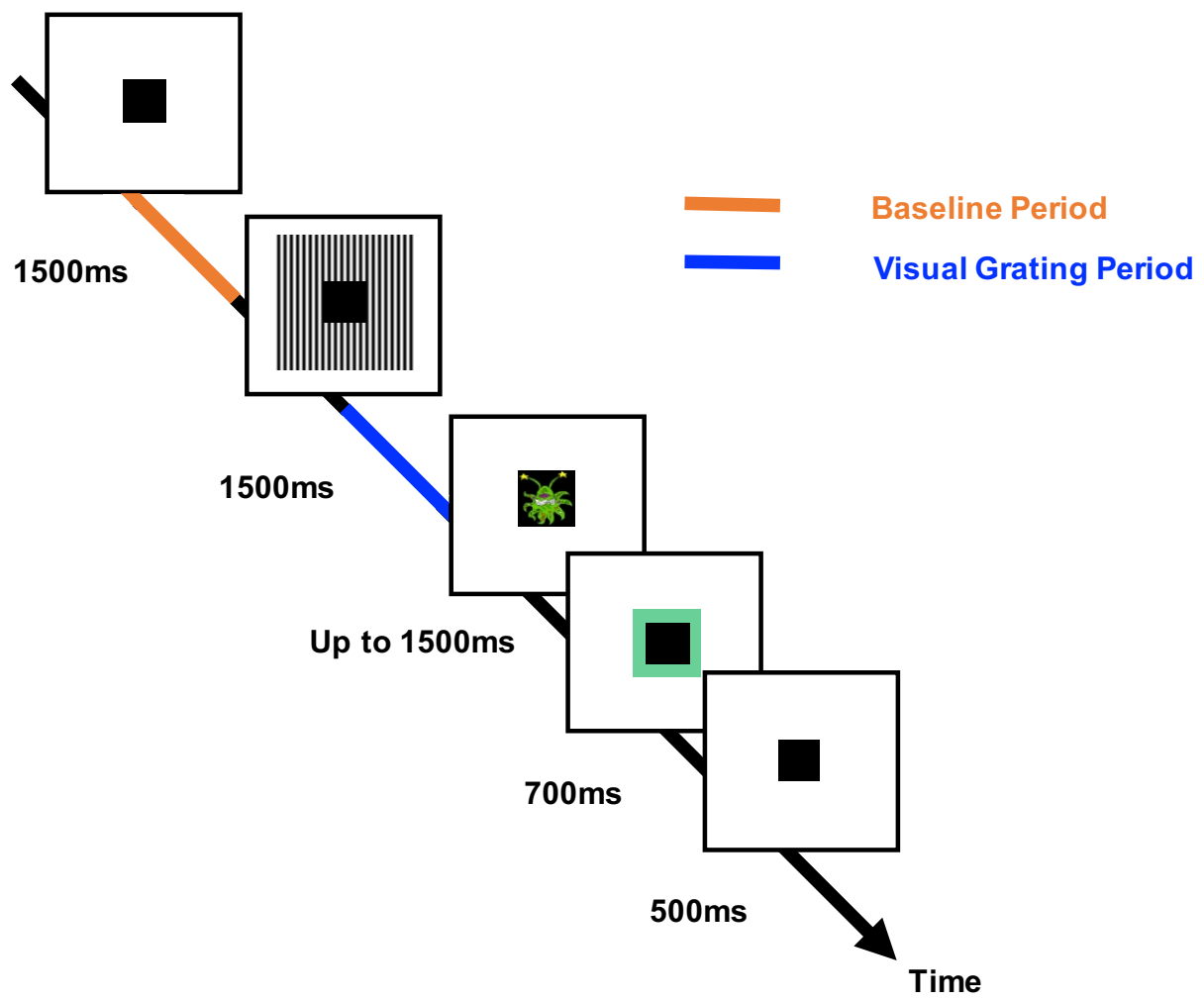

Figure 2.1: The structure of the engaging sensory paradigm. The times corresponding to the analysed baseline and visual grating periods are labelled in orange/blue respectively.

\subsubsection{MEG Acquisition}

MEG data were acquired using a 306-channel Neuromag MEG scanner (Vectorview, Elekta, Finland) made up of 102 triplets of two orthogonal planar gradiometers and one magnetometer. All recordings were performed inside a magnetically shielded room at a sampling rate of $1000 \mathrm{~Hz}$. Five head position indicator (HPI) coils were applied for continuous head position tracking, and visualised post-acquisition using an in-house Matlab script. For MEG-MRI coregistration purposes three fiducial points, the locations of the HPI coils and 300-500 points from the head surface were acquired using the integrated Polhemus Fastrak digitizer.

Visual stimuli were presented on a screen located $86 \mathrm{~cm}$ from participants (resulting in 2 cycles/degree for the visual grating), and auditory feedback through MEG-compatible earphones.

\subsubsection{Structural MRI}

A structural T1 brain scan was acquired for source reconstruction using a Siemens MAGNETOM Trio 3T scanner with a 32-channel head coil $(T E=2.18 \mathrm{~ms}, T R=2300 \mathrm{~ms}$, 
$\mathrm{TI}=1100 \mathrm{~ms}$, flip angle $=9^{\circ}, 192$ or 208 slices depending on head size, voxel-size $=$ $0.8 \times 0.8 \times 0.8 \mathrm{~cm})$.

\subsubsection{MEG-MRI Coregistration and 3D Cortical Mesh Construction}

MEG data were co-registered with participants MRI structural scan by matching the digitised head shape data with surface data from the structural scan (Jenkinson \& Smith, 2001). The aligned MRI-MEG image was used to create a forward model based on a single-shell description of the inner surface of the skull (Nolte, 2003), using the segmentation function in SPM8 (Litvak et al., 2011). The cortical mantle was then extracted to create a 3D cortical mesh, using Freesurfer v5.3 (Fischl, 2012), and registered to a standard fs_LR mesh, based on the Conte69 brain (Van Essen 2012), using an interpolation algorithm from the Human Connectome Project (Van Essen et al., 2012; instructions here: https://goo.gl/3HYA3L). Finally, the mesh was downsampled to 4002 vertices per hemisphere.

\subsubsection{Pre-processing}

MEG data were pre-processed using Maxfilter (temporal signal space separation, .9 correlation), which supresses external sources of noise from outside the head (Taulu \& Simola, 2006).

Further pre-processing steps were performed in Matlab 2014b using the open-source Fieldtrip toolbox v20161024 (Oostenveld et al., 2010; script: 1_preprocessing_elektra_frontiers_PAC.m). Firstly, for each participant the entire recording was band-pass filtered between $0.5-250 \mathrm{~Hz}$ (Butterworth filter, low-pass order 4 , high-pass order 3) and band-stop filtered $(49.5-50.5 \mathrm{~Hz} ; 99.5-100.5 \mathrm{~Hz})$ to remove residual $50 \mathrm{~Hz}$ power-line contamination and its harmonics. Data were then epoched into segments of $4 \mathrm{~s}$ (1.5s pre, $1.5 \mathrm{~s}$ post stimulus onset, with $0.5 \mathrm{~s}$ of padding either side) and each trial was demeaned and detrended. Trials containing artefacts (SQUID jumps, eye-blinks, head movement, muscle) were removed if the trial-by-channel (magnetomer) variance exceeded $8 \times 10^{-23}$, resulting in an average of 63.5 trials per condition, per participant. Sitespecific MEG channels containing large amounts of non-physiological noise were removed from all analyses (MEG channels: 0111, 0322, 2542, 0532).

\subsubsection{Source Analysis}

Source analysis was conducted using a linearly constrained minimum variance beamformer, LCMV, (Van Veen, van Drongelen, Yuchtman, \& Suzuki, 1997), which applies a spatial filter to the MEG data at each vertex of the 3D cortical mesh, in order to 
maximise signal from that location whilst attenuating signals elsewhere. Beamforming weights were calculated by combining the covariance matrix of the sensor data with leadfield information. Due to rank reduction following data cleaning with Maxfilter, the covariance matrix was kept at a rank below 64 components, which explained $99 \%$ of the variance. For all analyses, a common filter was used across baseline and grating periods, and a regularisation parameter of lambda $5 \%$ was applied.

Due to prior interest in the gamma and alpha-bands (Hoogenboom, Schoffelen, Oostenveld, Parkes, \& Fries, 2006; Michalareas et al., 2016; Muthukumaraswamy, Singh, Swettenham, \& Jones, 2010), the visual data were band-pass filtered (Butterworth filter) between $40-60 \mathrm{~Hz}$ (gamma) and $8-13 \mathrm{~Hz}$ (alpha), and source analysis was performed separately for each frequency band. To capture induced rather than evoked visual activity, a period of $0.3-1.5 \mathrm{~s}$ following stimulus onset was compared with a $1.2 \mathrm{~s}$ baseline period (1.5-0.3s before grating onset). The change in oscillatory power for each vertex was averaged across participants, interpolated onto a 3D mesh provided by the Human Connectome Project (Van Essen, 2012), and thresholded at a value which allowed the prominent patterns of power changes to be determined (see Figure 2.3, script:

2_get_source_power.m).

\subsubsection{Extracting Area V1 Time-series}

Trial time-courses were extracted from bilateral visual area $\mathrm{V} 1$, defined using a multimodal parcellation from the Human Connectome Project, which combined retinotopic mapping, T1/T2 structural MRI and diffusion-weighted MRI to accurately define the boundaries between cortical areas (Glasser et al., 2016; Figure 2.3C). To obtain a single spatial filter from this region, we performed a principle components analysis (PCA) on the concatenated filters from 182 vertices of bilateral V1, multiplied by the sensor-level covariance matrix, and extracted the first component. The sensor-level data was then multiplied by this spatial filter to obtain a V1-specific "virtual electrode" (script:

3_get_VE_frontiers_PAC.m), and the change in oscillatory power between grating and baseline periods was calculated from $1-100 \mathrm{~Hz}$, using a $0.5 \mathrm{~s}$ time window, sliding in steps of $20 \mathrm{~ms}$ and $\pm 8 \mathrm{~Hz}$ frequency smoothing (script: 4_calc_pow_change.m). It is important to note that while we decided to use a multimodal atlas, visual area V1 virtual electrode timeseries could also be defined using a more standard volumetric approach, for example the AAL atlas, which is included in the Fieldtrip toolbox (Oostenveld et al., 2010). 


\subsubsection{Phase Amplitude Coupling (PAC) Analysis}

V1 time-courses were examined for changes in alpha-gamma phase amplitude coupling (PAC). The general procedure is outlined in Figure 2.2. The first step was to obtain estimates of low frequency phase $\left(f_{\mathrm{p}}\right)$ and high frequency amplitude $\left(f_{\mathrm{a}}\right)$ for each trial using a fourth order, two-pass Butterworth filter, and then applying the Hilbert transform (Le Van Quyen et al., 2001). To avoid sharp edge artefacts, which can result in spurious PAC (Kramer, Tort, \& Kopell, 2008), the first $0.5 \mathrm{~s}$ and last $0.5 \mathrm{~s}$ of each trial was discarded.

The bandwidth of the filter used to obtain $f_{\mathrm{p}}$ and $f_{\mathrm{a}}$ is a crucial parameter in calculating PAC (Aru et al., 2015). The filters for extracting $f_{\mathrm{a}}$ need to be wide enough to capture the centre frequency \pm the modulating $f_{\mathrm{p}}$. So, for example, to detect PAC between $f_{\mathrm{p}}=13 \mathrm{~Hz}$ and $f_{\mathrm{a}}=60 \mathrm{~Hz}$, requires a $f_{\mathrm{a}}$ bandwidth of at least $13 \mathrm{~Hz}$ [47 73]. If this condition is not met, then PAC cannot be detected even if present (Dvorak \& Fenton, 2014). We therefore decided to use a variable bandwidth, defined as \pm 0.4 times the center frequency (e.g. for an amplitude of $60 \mathrm{~Hz}$, the bandwidth was $24 \mathrm{~Hz}$ either side [36 84]), which has been shown to improve the ability to detect PAC (Berman et al., 2012; Voloh et al., 2015). For alpha-band phase (maximum $13 \mathrm{~Hz}$ ), this allowed us to calculate PAC for amplitudes above $34 \mathrm{~Hz}$. The bandwidth for $f_{\mathrm{p}}$ was kept narrow ( $1 \mathrm{~Hz} \pm$ the center frequency), in order to extract sinusoidal waveforms. Furthermore, each trial was visually inspected to confirm that the $f_{\mathrm{p}}$ filtered oscillations were sinusoidal in nature. 


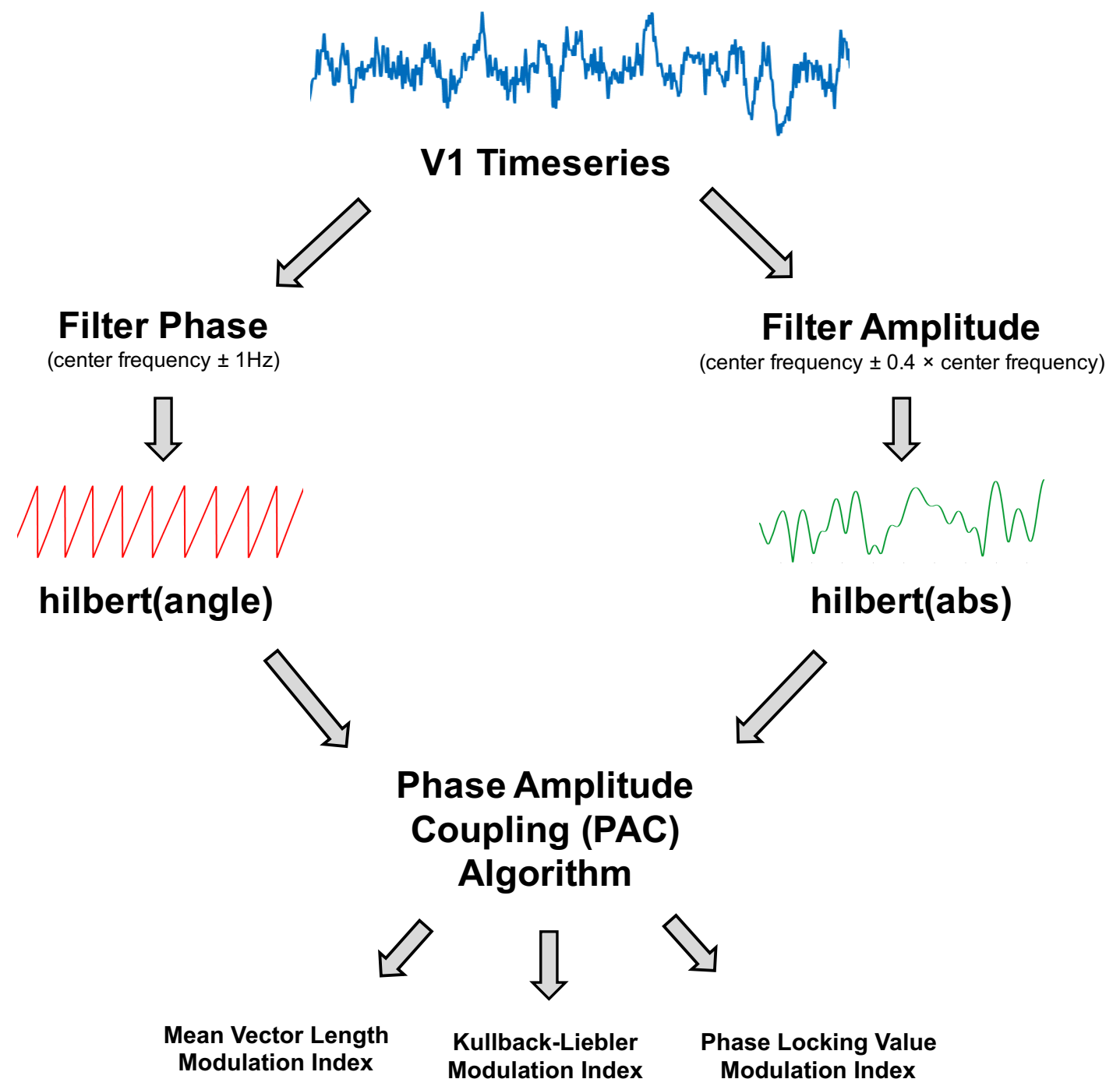

Figure 2.2: Illustration of the phase amplitude coupling (PAC) analysis procedure. The V1 time-series were filtered to obtain estimates of phase and amplitude, using a narrow $( \pm 1 \mathrm{~Hz})$ bandwidth for the phase and a variable bandwidth $( \pm 0.4$ times the centre frequency) for the amplitude. Phase and amplitude information were obtained via the Hilbert transform. The coupling between phase and amplitude was then quantified using Mean Vector Length, Kullback-Leiber or Phase Locking Value algorithms to produce a Modulation Index value.

Next, the coupling between $f_{\mathrm{p}}$ and $f_{\mathrm{a}}$ was quantified using four common PAC approaches : the Mean-Vector Length modulation index, originally described in Canolty et al., (2006); the Mean-Vector Length modulation index described in Özkurt \& Schnitzler

\footnotetext{
* Due to inconsistent naming practices, we refer to the quantitative value of PAC as the modulation index (MI) across all four approaches.
} 
(2011); the phase-locking value modulation index described in Cohen (2008); and the Kullback-Lieber modulation index described in Tort, Komorowski, Eichenbaum, \& Kopell (2010a). These approaches were selected due to their popularity in the MEG/EEG PAC literature (e.g. Bonnefond \& Jensen, 2015; Cho et al., 2015; Khan et al., 2013; Mathewson et al., 2011).

The mean vector length modulation index (MVL-MI-Canolty) approach estimates PAC from a signal with length $N$, by combining phase $(\phi)$ and amplitude information to create a complex-valued signal: $f_{a} e^{i\left(\phi f_{p}\right)}$ (Canolty et al., 2006), in which each vector corresponds to a certain time-point $(n)$. If the resulting probability distribution function is non-uniform, this suggests a coupling between $f_{\mathrm{p}}$ and $f_{\mathrm{a}}$, which can be quantified by taking the length of the average vector.

$$
M I=\left|\frac{1}{N} \sum_{n=1}^{N} f_{a}(n) e^{i\left(\phi f_{p}(n)\right)}\right|
$$

However, MI values from the MVL-MI-Canolty algorithm have been shown to partly reflect the power of $f_{\mathrm{a}}$ oscillations, rather than their coupling (Canolty \& Knight, 2010). Therefore, as an alternative to surrogate data, we applied a MVL-MI algorithm from Özkurt \& Schnitzler (2011), which includes a normalisation factor corresponding to the power of $f_{\mathrm{a}}$. Özkurt \& Schnitzler (2011) suggest that their algorithm is more resilient to measurement noise, and is therefore highly relevant for MEG data, which has an inherently lower signalto-noise ratio compared with invasive electrophysiological recordings (Goldenholz et al., 2009).

$$
M I=\frac{1}{\sqrt{N}} \frac{\left|\frac{1}{N} \sum_{n=1}^{N} f_{a}(n) e^{i\left(\phi f_{p}(n)\right)}\right|}{\sqrt{\frac{1}{N} \sum_{n=1}^{N} f_{a}(n)^{2}}}
$$

The PLV-MI-Cohen approach assumes that if PAC is present, the envelope of $f_{\mathrm{a}}$ should oscillate at the frequency corresponding to $f_{\mathrm{p}}$. The phase of $f_{\mathrm{a}}$ envelope can be obtained by applying the Hilbert transform (angle): $\phi f_{\mathrm{a}}$. The coupling between the low-frequency $\phi f_{\mathrm{p}}$ phase values and the phase of the amplitude envelope, $\phi f_{\mathrm{a}}$, can be quantified by calculating a phase locking value (PLV), in much the same way as determining phase synchronisation between electrophysiological signals. 


$$
M I=\left|\frac{1}{N} \sum_{n=1}^{N} e^{i\left(\phi f_{p}(n)-\phi f_{a}(n)\right.}\right|
$$

Finally, the KL-MI-Tort approach estimates PAC by quantifying the amount of deviation in amplitude-phase distributions. This involves breaking $f_{\mathrm{p}}$ into 18 bins, and calculating the mean amplitude within each phase bin, normalised by the average value across all bins. Although the number of phase bins chosen is arbitrary, the specific number $(9,18$ or 36$)$ does not seem to influence PAC estimation (see Appendix 1). The modulation index is calculated by comparing the amplitude-phase distribution $(P)$ against the null hypothesis of a uniformly amplitude-phase distribution (Q).

$$
M I=\frac{D(P, Q)}{\log \left(N_{\text {bins }}\right)}
$$

Mathematically, this is computed using the Kullbeck-Leiber distance (D), related to Shannon's entropy.

$$
D(P, Q)=\sum_{i_{b i n}=1}^{N} P\left(i_{b i n}\right) \cdot \log \left(\frac{P\left(i_{b i n}\right)}{Q\left(i_{b i n}\right)}\right)
$$

Using these four approaches (MVL-MI-Canolty; MVL-MI-Özkurt; KL-MI-Tort; PLV-MICohen) we calculated PAC between phases $7-13 \mathrm{~Hz}$ (in $1 \mathrm{~Hz}$ steps) and amplitudes 34$100 \mathrm{~Hz}$ (in $2 \mathrm{~Hz}$ steps), for the time-period $0.3-1.5$ s following grating presentation and a $1.2 \mathrm{~s}$ baseline period. PAC values were calculated separately for each trial and then averaged to obtain a single MI value per amplitude and phase. This was repeated using surrogate data, created by shuffling trial and phase-carrying information (200 surrogates), to normalise MI values. On a PC with 32GB of RAM, and Intel(R) Core ${ }^{\mathrm{TM}} \mathrm{i7}-4790$ processor, the computation time for these procedures was 4.5 hours (script: 5_visual_PAC_four_methods.m).

To assess changes in the strength of PAC between the grating and baseline periods, the comodulograms were compared using non-parametric cluster-based statistics, which have been shown to adequately control the type-I error rate for electrophysiological data (Maris \& Oostenveld, 2007). First, an uncorrected dependent-samples t-test was performed (grating versus baseline), and all MI values exceeding a 5\% significance threshold were grouped into clusters. The maximum t-value within each cluster was carried forward. Next, a null distribution was obtained by randomising the condition label (grating/baseline) 1000 times and calculating the largest cluster-level t-value for each permutation. The maximum 
t-value within each original cluster was then compared against this null distribution, with values exceeding a threshold of $p<.05$ deemed significant.

\subsubsection{Sinusoidal Oscillations}

One major issue in cross-frequency coupling analysis is the presence of non-sinusoidal sawtooth-like oscillations (Cole et al., 2017; Jensen et al., 2016), which can result in spurious estimates of PAC (Lozano-Soldevilla et al., 2016). This property of oscillations can be quantified by calculating the time taken from trough to peak (rise-time), peak to trough (decay-time), and the ratio between these values (Cole \& Voytek, 2017; Dvorak \& Fenton, 2014). We therefore calculated this ratio for the visual $\mathrm{V} 1$ data from $7-13 \mathrm{~Hz}$, and performed a t-test to check for differences in non-sinusoidal oscillations between grating and baseline periods (script: 6_check_non_sinusoidal.m).

\subsubsection{Simulated PAC Analysis}

To investigate the validity of the four PAC approaches, we constructed 1.2 seconds of simulated data with known alpha-gamma PAC $\left(f_{\mathrm{p}}=10 \mathrm{~Hz} ; f_{\mathrm{a}}=50-70 \mathrm{~Hz}\right.$; code adapted from Kramer et al., (2008) and Özkurt \& Schnitzler (2011)) and added a random level of noise (signal-to-noise ratio $>-11.5 \mathrm{~dB}$ ). Comodulograms were produced using the four PAC algorithms on 64 trials of simulated data. Using the same code, we also investigated how the four algorithms were affected by trial length $(0.1-10$ s in 0.1 second steps, script: 7_simulated_PAC_analysis.m).

\subsection{Results}

\subsubsection{Source Localisation}

In order to establish patterns of oscillatory power changes following presentation of the visual grating, gamma-band $(40-60 \mathrm{~Hz})$ and alpha-band power $(8-13 \mathrm{~Hz})$ were localised for a $0.3-1.5$ s period post-stimulus presentation. Results for the gamma-band, Figure 2.3A, show an increase in oscillatory power which localises to the ventral occipital cortex (Hoogenboom et al., 2006). Results for the alpha band, Figure 2.3B, showed a general decrease in power, located primarily in occipital areas, but extending into temporal and parietal regions. The more widespread spatial pattern could reflect on-going upstream processes triggered by the appearance of the grating, for example anticipation of the upcoming target (Stenner, Bauer, Haggard, Heinze, \& Dolan, 2014). 


\subsubsection{Visual Area V1 Power Changes}

Time courses from area $\mathrm{V} 1$ were extracted, Figure 2.3C, and the change in oscillatory power between grating and baseline periods from $1-100 \mathrm{~Hz}$ was calculated, Figure 2.3D. Whilst results show individual variability in peak frequencies and the strength of oscillatory power, on average, activity within visual area $\mathrm{V} 1$ displays a reduction in alpha/beta power $(8-20 \mathrm{~Hz})$, and an increase in gamma power $(40-70 \mathrm{~Hz})$. The MEG data, therefore display well-established patterns of alpha and gamma-band event-related synchronisation and desynchronisation within visual area V1 (Bonnefond \& Jensen, 2015; Hoogenboom et al., 2006; Michalareas et al., 2016), which is a crucial first step in calculating reliable estimates of PAC (Aru et al., 2015).

\subsubsection{Alpha-Gamma PAC}

Visual area V1 responses were next examined for changes in alpha-gamma PAC.

Specifically, we set out to test whether the coupling between alpha-band phase and gamma-band amplitude was altered during presentation of the visual grating. Phaseamplitude comodulograms were produced between a range of phase frequencies (7$13 \mathrm{~Hz}$ ) and amplitude frequencies $(34-100 \mathrm{~Hz})$, using the four algorithms described in Methods: MVL-MI-Canolty; MVL-MI-Özkurt; PLV-MI-Cohen and KL-MI-Tort. Grating and baseline comodulograms were compared using cluster-based non-parametric statistics (Maris \& Oostenveld, 2007). 
A

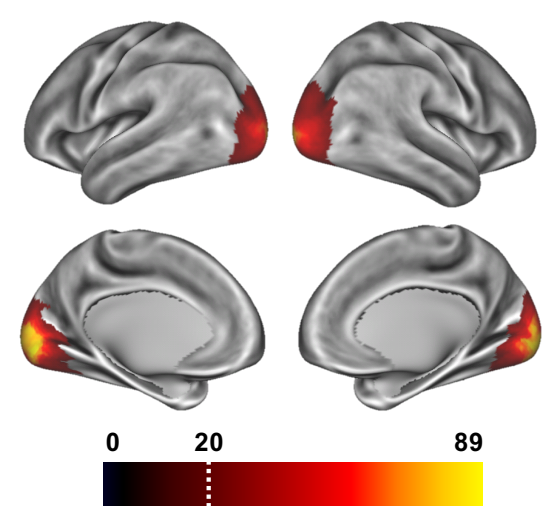

$40-60 \mathrm{~Hz} \%$ power change

C

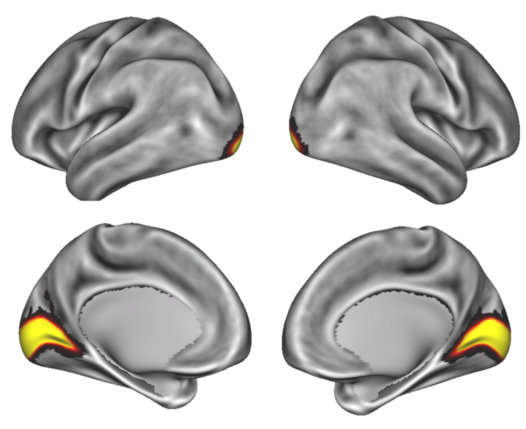

B

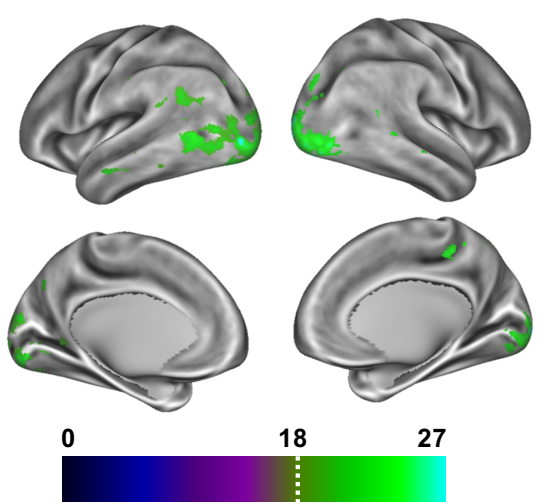

$8-13 \mathrm{~Hz} \%$ power change

D

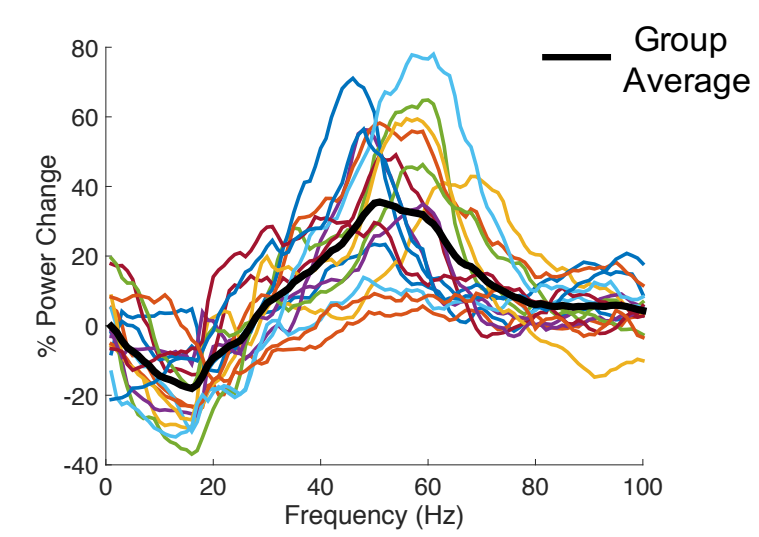

Figure 2.3: Whole-brain oscillatory power changes following the presentation of the visual grating are marked by (A) increases in the gamma-band $(40-60 \mathrm{~Hz})$ and $(B)$ decreases in the alpha-band $(8-13 \mathrm{~Hz})$, localised primarily in the ventral occipital cortex. Power maps were thresholded at a value which allowed prominent patterns of power changes to be determined, indicated by the white dotted line. Time-courses were extracted from bilateral visual area V1, defined using the atlas region shown in (C) from the HCP-MMP 1.0 parcellation (Glasser et al., 2016). (D) These V1 responses showed reductions in alpha/beta power and increases in gamma-band $(40-70 \mathrm{~Hz})$ power. 
Results are shown in Figure 2.4A. Using the MVL-MI-Canolty algorithm, there was a significant increase in alpha-gamma PAC over a large proportion of the comodulogram, between $40-100 \mathrm{~Hz}$ and $7-13 \mathrm{~Hz}$, with a peak at $50-70 \mathrm{~Hz}$ amplitude and $9-10 \mathrm{~Hz}$ phase. This large area of significantly increased PAC is likely to reflect, in part, power increases in the gamma-band (Canolty et al., 2006). The alternative MVL-MI- Özkurt algorithm, which normalises MI values by the high-frequency oscillatory power, displayed a smaller area of significant coupling, with increased PAC between an amplitude of $50-70 \mathrm{~Hz}$ and phase of $10 \mathrm{~Hz}$. There was also a similar cluster of significantly increased PAC between 9$11 \mathrm{~Hz}$ and $50-70 \mathrm{~Hz}$ using the PLV-MI-Cohen approach. The KL-MI-Tort results showed clusters of increased PAC between amplitudes of $50-100 \mathrm{~Hz}$ and phases of $9-10 \mathrm{~Hz}$, but decreased PAC between amplitudes of $60-90 \mathrm{~Hz}$ and phases of $12-13 \mathrm{~Hz}$. However, none of these clusters passed a significance threshold of $p<0.05$ (two-tailed). Similar results were obtained after normalising MI values with surrogate data, Figure 2.4B.

\subsubsection{Non-Sinusoidal Oscillations}

To determine whether our alpha-gamma PAC results were driven by differences in the sinusoidal properties of oscillations between baseline and grating periods, the ratio between oscillatory rise-time and decay-time was calculated. For the alpha phase frequencies $(7-13 \mathrm{~Hz})$, there was no difference in this ratio (all frequencies $p>05$ ), suggesting that our results are unlikely to be caused by increased non-sinusoidal sawtooth-like properties of alpha oscillations during stimulus period compared to baseline.

\subsubsection{Simulated PAC}

To further validate our PAC results, we generated simulated data with known alphagamma coupling $(10-11 \mathrm{~Hz}$ phase, $50-70 \mathrm{~Hz}$ amplitude). Using the same MATLAB code as for the MEG data, we were able to successfully detect this alpha-gamma PAC using the MVL-MI-Canolty, MVL-MI-Özkurt, PLV-MI-Cohen and KL-MI-Tort algorithms, Figure 2.5A. By varying the trial length of the simulated data, we found that PAC values were affected by trial length, with data segments under 1 second producing artificially inflated PAC, Figure 2.5B. 
A

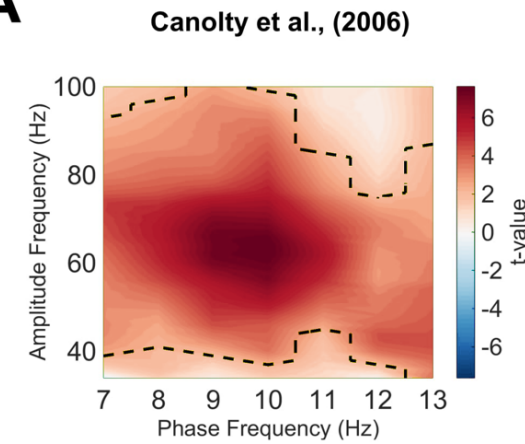

PLV-MI

Cohen et al., (2008)

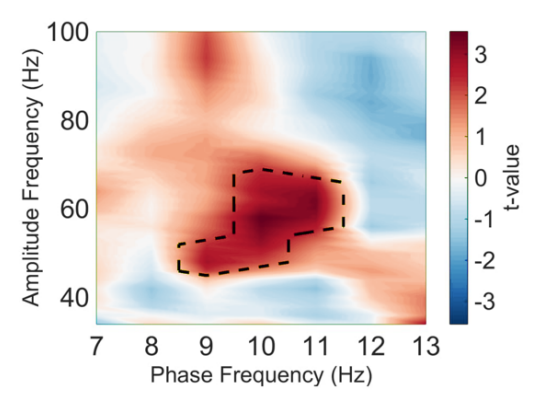

MVL-MI

Özkurt \& Schnitzler (2010)

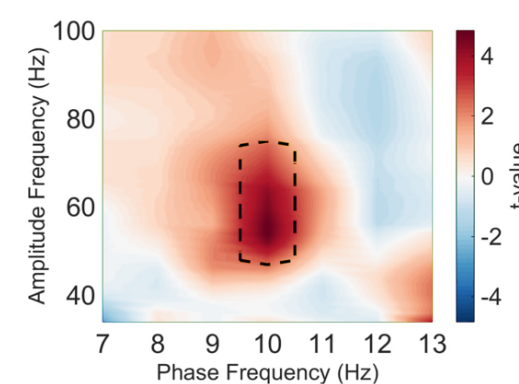

KL-MI

Tort et al., (2010a)

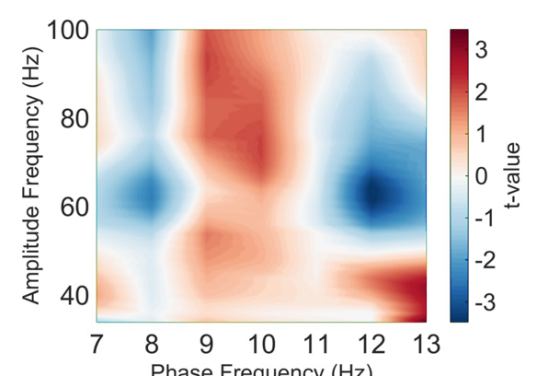

MVL-MI

Canolty et al., (2006)

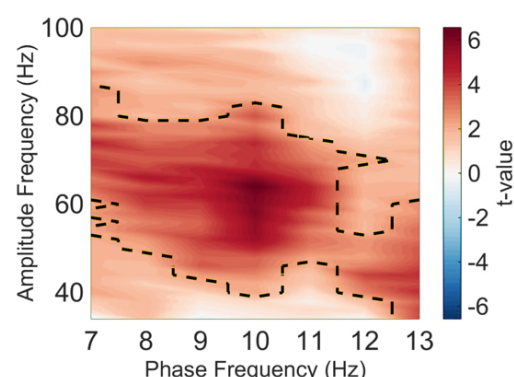

PLV-MI

Cohen et al., (2008)

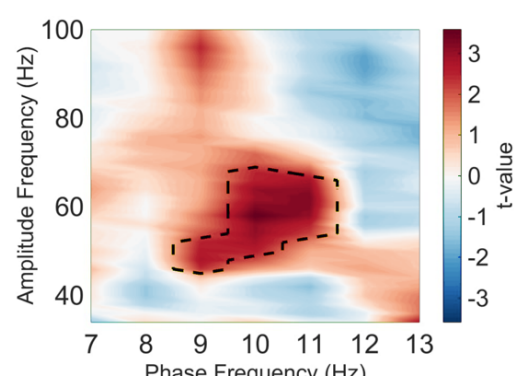

MVL-MI

Özkurt \& Schnitzler (2010)

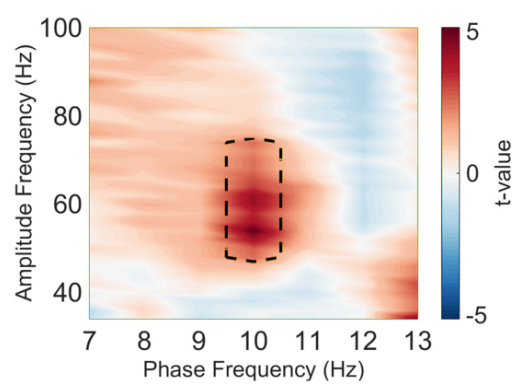

KL-MI

Tort et al., (2010a)

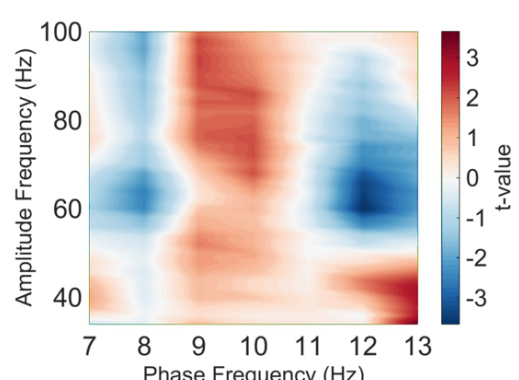

Figure 2.4: Phase-amplitude comodulograms produced by statistically comparing modulation index (MI) values from 0.3-1.5s post-grating onset to a $1.2 \mathrm{~s}$ baseline period, using four separate approaches. Comodulograms for $(A)$ raw MI values and $(B)$ MI values normalised by surrogate data are shown separately. The black dotted line represents significantly different phase-amplitude coupling frequencies ( $p<.05$; for details of non-parametric cluster-based statistics see Methods). 
A

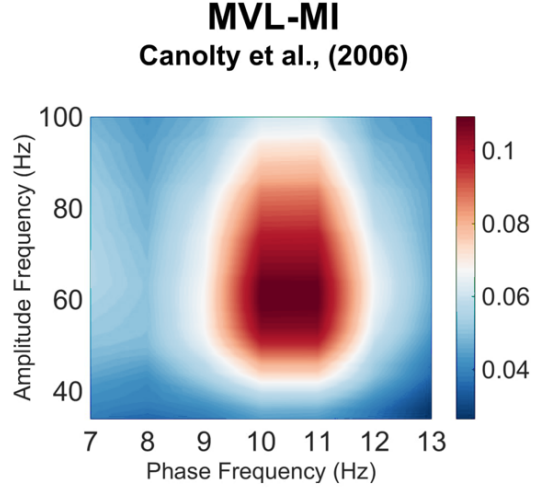

PLV-MI

Cohen et al., (2008)

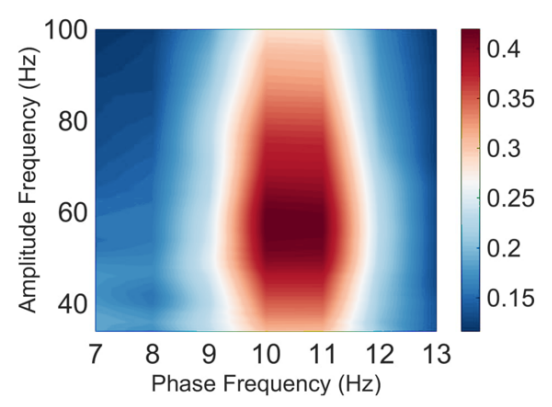

MVL-MI

Özkurt \& Schnitzler (2010)

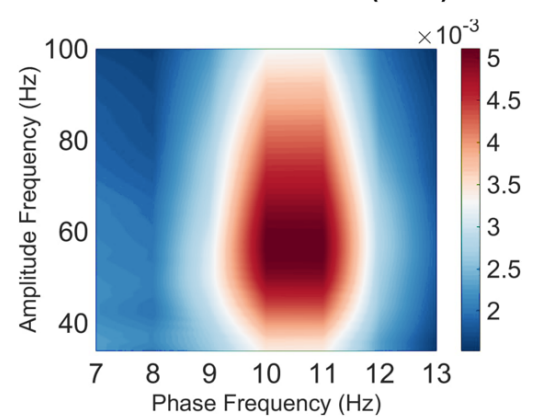

KL-MI

Tort et al., (2010a)

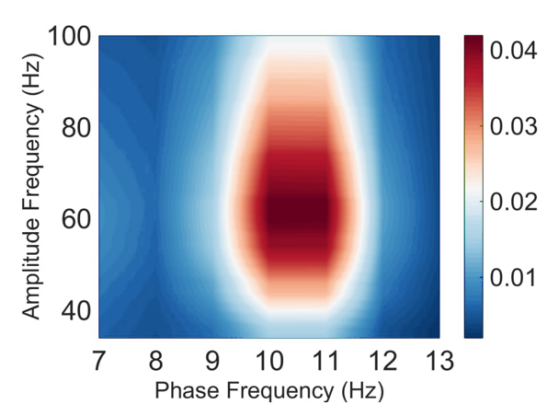

B

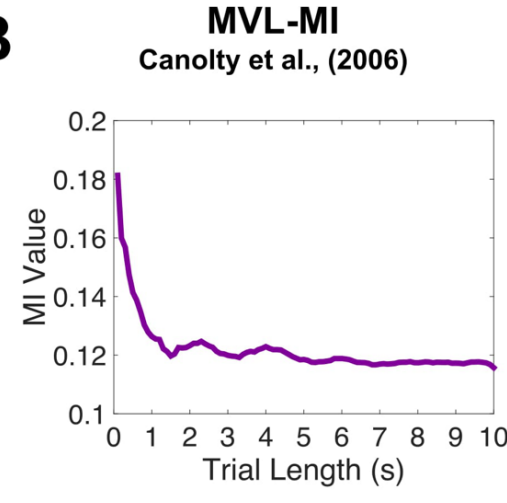

PLV-MI

Cohen et al., (2008)

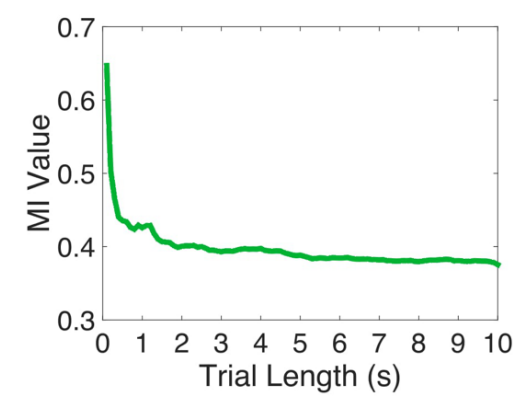

MVL-MI

Özkurt \& Schnitzler (2010)

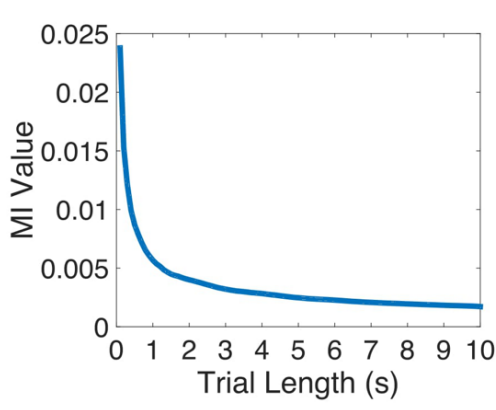

KL-MI

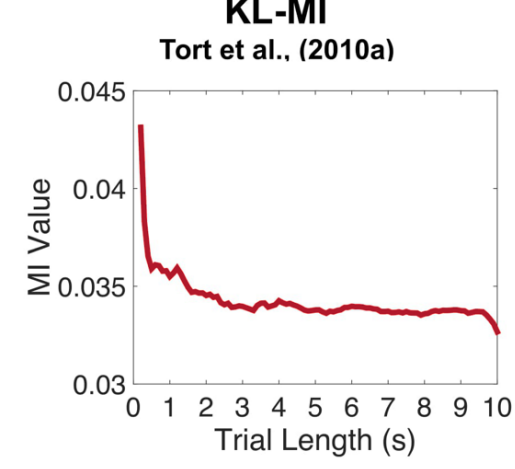

Figure 2.5: Results of the simulated PAC analysis. (A) Phase-amplitude comodulograms produced using the MVL-MI-Canolty, MVL-MI-Özkurt, PLVMI-Cohen and KL-MI-Tort algorithms were able to successfully detect the 1.2 seconds of simulated coupling between 10Hz phase and $50-70 \mathrm{~Hz}$ amplitude. (B) The coupling between $10 \mathrm{~Hz}$ phase and $60 \mathrm{~Hz}$ amplitude was calculated as a function of simulated data trial length. For trial data under 1 second, all four algorithms produced artificially inflated PAC. 


\subsection{Discussion}

This chapter has outlined various steps involved in the detection and validation of phase amplitude coupling (PAC) in a visual MEG dataset (data shared at:

https://doi.org/10.6084/m9.figshare.c.3819106.v1), utilising the open-source Fieldtrip toolbox (Oostenveld et al., 2010) and customised Matlab scripts, see

https://github.com/neurofractal/sensory PAC. We first confirmed that presentation of the visual grating was accompanied by decreases in alpha power $(8-13 \mathrm{~Hz})$ and increases in gamma power $(>40 \mathrm{~Hz})$ within visual area $\mathrm{V} 1$. Although this may seem redundant given the wealth of evidence for alpha and gamma oscillations in visual processing (Bonnefond \& Jensen, 2015; Hoogenboom et al., 2006; Michalareas et al., 2016), it is crucial to establish clear increases/decreases in the power spectrum at two distinct frequencies as a first step in MEG-PAC analysis (Aru et al., 2015; Hyafil et al., 2015). Using four PAC algorithms, we showed that visual responses obtained from area $\mathrm{V} 1$ displayed a general increase in alpha-gamma PAC as expected (Bonnefond \& Jensen, 2015; Spaak et al., 2012; Voytek et al., 2010). However, it is important to note that specific patterns of coupling depended on the algorithm selected. The MVL-MI-Canolty algorithm showed large increases in PAC during the grating period, covering almost the entire alpha \& gamma frequency ranges, most likely as a result of $\mathrm{Ml}$ values being biased by increases in high-frequency power following presentation of the visual grating (Canolty et al., 2006). This approach is therefore less suitable for detecting PAC between separate periods of data and/or trials. The MVL-MI-Özkurt algorithm, which normalises the MI value by high amplitude power, along with the PLV-MI-Cohen algorithm produced a much more constrained pattern of significant alpha-gamma PAC, with peaks between $9-11 \mathrm{~Hz}$ phase and $50-70 \mathrm{~Hz}$ amplitude. Whilst the KL-MI-Tort approach also showed a general increase in alpha-gamma PAC around $9-11 \mathrm{~Hz}$, none of the phase-amplitude clusters reached significance. This may be due to the relatively low number of trials used in the experiment, variations in the peak alpha and gamma oscillatory frequencies (Muthukumaraswamy, Edden, Jones, Swettenham, \& Singh, 2009), combined with the fact that the KL-MI-Tort approach is relatively conservative (van Driel, Cox, \& Cohen, 2015). More generally, it is important to emphasise that all four PAC metrics are highly sensitive to a range of factors (Aru et al., 2015; Dvorak \& Fenton, 2014), which are often hard to control (Berman et al., 2012), resulting in both type I and type II statistical errors.

One such issue is the presence of non-sinusoidal sawtooth-like oscillations in electrophysiological data, which can result in spurious PAC (Lozano-Soldevilla et al., 2016), especially when phase is obtained with wide band-pass filters. By computing the ratio between rise-time and decay-time of alpha oscillations within area $\mathrm{V} 1$, we showed 
that non-sinusoidal oscillations did not differ between baseline and grating periods, and are unlikely to account for our results. Another issue in trial-based PAC analysis is data length, with some previous reports suggesting that 10 seconds or more is required for detecting theta-gamma coupling (Aru et al., 2015; Dvorak \& Fenton, 2014). However, using simulated alpha-gamma PAC we determined that 1 second of data was sufficient to obtain stable estimates to quantify PAC. This can be seen in Figure 2.5B where the MI value / trial length curve stabilises around 1s, meaning that increasing the length of the data beyond $1 \mathrm{~s}$ results in the same PAC value.

\subsubsection{Practical Considerations for PAC analysis}

Cross-frequency coupling is gaining significant interest within the electrophysiological community (Aru et al., 2015; Canolty \& Knight, 2010; Dvorak \& Fenton, 2014; Hyafil et al., 2015), and therefore it is important for researchers to consider the methodological pitfalls and caveats which commonly arise during PAC analysis. Firstly, due to the presence of edge artefacts at the start and end of time-series created by bandpass filtering, which can result in artefactual PAC (Kramer et al., 2008), sufficient padding should be included around trials. Concatenating data from separate trials to create longer data segments results in similar edge artefacts (Kramer et al., 2008), and should be avoided. Secondly, if the bandwidth of the filter used to extract the amplitude does not contain the side-bands of the modulating phase frequency, PAC cannot be detected even if present (Dvorak \& Fenton, 2014). The use of a variable band-pass filter which scales with amplitude frequency, can alleviate this issue and improve the sensitivity of detecting PAC (Berman et al., 2012; Voloh et al., 2015). Thirdly, periods which contain non-stationary periods should be avoided. This includes sensory evoked potentials which induce correlations between frequency bands via phase reset (Sauseng et al., 2007), and can be misinterpreted as PAC (Aru et al., 2015). For this reason, we did not analyse the first $0.3 \mathrm{~s}$ following visual grating presentation, due to the presence of visual evoked potentials (Di Russo, Martínez, Sereno, Pitzalis, \& Hillyard, 2002). Fourth, given that PAC algorithms produce values ranging from 0 to 1 , data are commonly not normally distributed, and therefore the use of non-parametric statistics is paramount. Whilst surrogate data are often employed (Aru et al., 2015; Tort et al., 2010a), this may not be possible where data are organised into short trials and temporal correlations between surrogate and true timeseries are high (Dvorak \& Fenton, 2014). Therefore, to assess changes in PAC, using a baseline period or contrasting between conditions, combined with non-parametric statistics may prove to be a useful alternative for sensory neurocognitive research.

\subsubsection{Limitations}


This study has compared four PAC algorithms (Canolty et al., 2006; Cohen, 2008; Özkurt \& Schnitzler, 2011; Tort et al., 2010a), which are among the most commonly used approaches in sensory EEG/MEG research (Bonnefond \& Jensen, 2015; Cho et al., 2015; Khan et al., 2013; Mathewson et al., 2011). However these only comprise a small subset of the available algorithms designed to quantify PAC (Canolty \& Knight, 2010; Hyafil et al., 2015). There have also been advances in measuring transient changes in PAC (Dvorak \& Fenton, 2014), directed PAC (Jiang, Bahramisharif, van Gerven, \& Jensen, 2015) and algorithms designed for spontaneous neural activity (Florin \& Baillet, 2015; Weaver et al., 2016). A more comprehensive evaluation of algorithms and their application to real-world electrophysiological data is beyond the scope of this chapter, but would nevertheless benefit the field of cross-frequency coupling. Secondly, in order to detect alpha-gamma PAC within visual area $V 1$, we used a broad filter bandwidth, defined as \pm 0.4 times the amplitude centre-frequency. Consequently, the alpha-gamma comodulograms will be unable to differentiate between adjacent gamma sub-bands, which have been proposed to fulfil differing neurocognitive roles (Bosman, Lansink, \& Pennartz, 2014; Buzsáki \& Wang, 2012), and patterns of PAC (Vaz et al., 2017). However, for the visual MEG data presented here, there was only an increase in gamma power within one band $(40-70 \mathrm{~Hz})$, and therefore the smearing of adjacent sub-bands is unlikely. Finally, we have focussed on PAC within the visual cortex, which is known to display sinusoidal alpha oscillations (Tort et al., 2010b). However, there are many examples of non-sinusoidal brain oscillations caused by physiological neuronal spiking patterns (Fontanini \& Katz, 2005), including hippocampal theta $(4-8 \mathrm{~Hz})$ and sensorimotor $\mathrm{mu}(9-11 \mathrm{~Hz})$ rhythms (LozanoSoldevilla et al., 2016; Scheffer-Teixeira \& Tort, 2016), which are indicative of behaviour and disease states (Cole \& Voytek, 2017). Therefore, whilst non-sinusoidal oscillations generate spurious PAC, this does not mean that these oscillations are uninteresting, but simply that common PAC algorithms, such as the ones employed in this chapter, are illsuited for these scenarios. Where non-sinusoidal oscillations are present, PAC analysis could proceed by correcting for non-uniform phase distributions (e.g van Driel, Cox, \& Cohen, 2015) in order to disentangle nested oscillations from neural spiking (Vaz et al., 2017).

\subsubsection{Conclusion}

In conclusion, we have outlined the key analysis steps for detecting changes in alphagamma PAC during sensory processing, using an example visual MEG dataset. While alpha-gamma PAC was shown to increase, relative to baseline, the specific patterns of alpha-gamma coupling depended upon the specific algorithm employed. Follow-up analyses showed that these results were not driven by non-sinusoidal oscillations or 
insufficient data. In future, we hope that a variety of PAC algorithms will be implemented alongside existing open-source MEG toolboxes (Gramfort et al., 2014; Oostenveld et al., 2010; Tadel, Baillet, Mosher, Pantazis, \& Leahy, 2011), with detailed guidance and advice, so that PAC can form a natural analysis step in electrophysiological research.

\section{Author Contributions}

RS, KK \& GR co-designed the study. RS collected the data, carried out the analysis, organised the data and code for sharing and was the primary author of this chapter.

\section{Conflict of Interests Statement}

The authors wish to declare the research was conducted in the absence of any commercial or financial relationships that could be construed as a potential conflict of interest.

\section{Acknowledgments}

We wish to thank: the volunteers who gave their time to participate in this study; JanMathijs Schoffelen for providing MATLAB code; Gerard Gooding-Williams and Shu Yau for help with MRI data acquisition; and the Wellcome and Dr Hadwen Trusts for supporting MEG scanning costs. Robert Seymour was supported by a cotutelle PhD studentship from Aston University and Macquarie University. 


\title{
Chapter 3: Dysregulated Oscillatory Connectivity in the Visual System in Autism Spectrum Disorder
}

\author{
Seymour, R.A ${ }^{1,2,3}$., Rippon, ${ }^{1}{ }^{1}$, Gooding-Williams, $G^{1}$,. \\ Schoffelen, J.M $\mathrm{M}^{4} \&$ Kessler, $\mathrm{K}^{1}$.
}

${ }^{1}$ Aston Brain Centre, School of Life and Health Sciences, Aston University, Birmingham, B4 7ET.

${ }^{2}$ ARC Centre of Excellence in Cognition and Its Disorders, Macquarie University, Sydney, Australia, 2109.

${ }^{3}$ Department of Cognitive Science, Macquarie University, Sydney, Australia, 2109.

${ }^{4}$ Radboud University Nijmegen, Donders Institute for Brain, Cognition and Behaviour, Center for Cognitive Neuroimaging, The Netherlands.

Please note, a version of this chapter has been uploaded to the pre-print server BioRxiv:

Seymour, R. A., Rippon, G., Gooding-Williams, G., Schoffelen, J. M., \& Kessler, K. (2018). Dysregulated Oscillatory Connectivity in the Visual System in Autism Spectrum Disorder. bioRxiv, 440586.

\subsection{Abstract}

Autism Spectrum Disorder is increasingly associated with atypical perceptual and sensory symptoms. Here we explore the hypothesis that aberrant sensory processing in Autism Spectrum Disorder could be linked to atypical intra- (local) and inter-regional (global) brain connectivity. To elucidate oscillatory dynamics and connectivity in the visual domain we used magnetoencephalography and a simple visual grating paradigm with a group of 18 adolescent autistic participants and 18 typically developing controls. Both groups showed 
similar increases in gamma $(40-80 \mathrm{~Hz})$ and decreases in alpha $(8-13 \mathrm{~Hz})$ frequency power in occipital cortex. However, systematic group differences emerged when analysing local and global connectivity in detail. Firstly, directed connectivity was estimated using nonparametric Granger causality between visual areas V1 and V4. Feedforward V1-to-V4 connectivity, mediated by gamma oscillations, was equivalent between Autism Spectrum Disorder and control groups, but importantly, feedback V4-to-V1 connectivity, mediated by alpha $(8-14 \mathrm{~Hz})$ oscillations, was significantly reduced in the Autism Spectrum Disorder group. This reduction was positively correlated with autistic quotient scores, consistent with an atypical visual hierarchy in autism, characterised by reduced top-down modulation of visual input via alpha-band oscillations. Secondly, at the local level in V1, coupling of alpha-phase to gamma amplitude (alpha-gamma phase amplitude coupling) was reduced in the Autism Spectrum Disorder group. This implies dysregulated local visual processing, with gamma oscillations decoupled from patterns of wider alpha-band phase synchrony, possibly due to an excitation-inhibition imbalance. More generally, these results are in agreement with predictive coding accounts of neurotypical perception and indicate that visual processes in autism are less modulated by contextual feedback information.

\subsection{Introduction}

As reviewed in Chapter 1, the current literature regarding oscillatory power and phasesynchrony in autism are very heterogenous, with both increases and decreases reported (for more discussion, see Kessler et al., (2016); Simon \& Wallace (2016)). Our group and others have recently suggested that these inconsistencies might be reconciled by shifting the notion from oscillatory power towards considering the oscillation-mediated functional connectivity at the global and local scales (see Chapter 1 Section 1.5; Kessler et al., 2016; Simon \& Wallace, 2016).

Functional connectivity has been proposed as a unifying framework for autism, with the predominant theory emerging from $\mathrm{fMRI}$ data being a global reduction but local increase in connectivity (Courchesne \& Pierce, 2005; Hughes, 2007). Recent M/EEG research has supported the first of these claims with reductions in global connectivity during set-shifting, slit-viewing, emotional face processing and whole-brain resting state studies (Doesburg, Vidal, \& Taylor, 2013; Khan et al., 2013; Kitzbichler et al., 2015; Peiker et al., 2015). These reductions in connectivity are generally tied to feedback processes, located within the frontal lobes, and mediated by oscillations in theta $(3-6 \mathrm{~Hz})$, alpha $(8-13 \mathrm{~Hz})$ and betabands $(13-30 \mathrm{~Hz})$. Interestingly, a recent study showed that during somatosensory stimulation, feedforward connectivity from primary to secondary somatosensory cortex is increased in ASD (Khan et al., 2015). This suggests that feedforward pathways in the 
autistic brain may be over-compensating for the lack of feedback connectivity. At the local level, M/EEG studies have not supported the local increase in connectivity reported using fMRI (Keown et al., 2013). An emerging biologically-relevant proxy for local connectivity is the coupling of oscillations from different frequency-bands, measured from within the same region, termed cross-frequency coupling (Canolty \& Knight, 2010; Kessler et al., 2016; Khan et al., 2013; Seymour, Rippon, \& Kessler, 2017). In particular, phaseamplitude coupling (PAC) has been proposed to act as a mechanism for the dynamic coordination of brain activity over multiple spatial scales, with the amplitude of highfrequency gamma-band activity within local ensembles coupled to large-scale patterns of low-frequency phase synchrony (Bonnefond, Kastner, \& Jensen, 2017). Alpha-gamma PAC is also closely tied to the balance between excitatory and inhibitory (E-I) populations of neurons (Mejias, Murray, Kennedy, \& Wang, 2016), which is affected in autism (Rubenstein \& Merzenich, 2003). One previous study has reported dysregulated alphagamma PAC in the fusiform face area during emotional face processing in a group of autistic adolescents (Khan et al., 2013). Interestingly, local PAC was related to patterns of long-range alpha hypoconnectivity in autism, suggesting that local and long-range connectivity are concurrently affected. Altogether, oscillation-based functional connectivity in autism could be characterised by local dysregulation and global hypoconnectivity, see Chapter 1 and Kessler et al., (2016) for more discussion.

Within the context of visual processing, this view leads to several hypotheses. Invasive electrocorticography (ECoG) recordings in macaques and non-invasive MEG in humans have shown that visual oscillations in different frequency bands have distinct cortical communication profiles. Gamma-band oscillations pass information up the visual hierarchy, in a feedforward manner, whereas alpha-band and beta-band oscillations mediate feedback connectivity, down the cortical hierarchy (Bastos, Vezoli, et al., 2015; Kerkoerle et al., 2014; Michalareas, Vezoli, van Pelt, et al., 2016). Long-range alpha/beta connectivity has also been linked with top-down attentional processes during visual perception via the regulation of local gamma oscillations (Klimesch, 2012; Richter, Thompson, Bosman, \& Fries, 2017) and of local alpha-gamma PAC (Chacko et al., 2018). If autism is associated with alterations to directed functional connectivity (Khan et al., 2015), we hypothesised reduced feedback connectivity within the visual system, mediated by oscillations in the alpha band, but potentially increased feedforward connectivity in the gamma band (Kessler et al., 2016). At the local level, neurotypical visual processing is accompanied by increases in alpha-gamma PAC, thought to arise through the coupling of excitatory and inhibitory neurons in the infragranular and supragranular layers of visual cortex (Spaak, Bonnefond, Maier, Leopold, \& Jensen, 2012). Given an E-I imbalance in 
autism and reported local dysregulation of cortical activity, we hypothesised reduced alpha-gamma PAC within primary visual cortex (Kessler, Seymour, \& Rippon, 2016; Khan et al., 2013). Finally, if top-down alpha connectivity has a modulatory effect on local activity within the visual system, then alpha-gamma PAC, in V1, should reveal a systematic relationship with top-down alpha connectivity, from V4.

We tested these hypotheses using MEG, which combines excellent temporal resolution with sophisticated source localisation techniques (Hillebrand \& Barnes, 2005). A group of 18 adolescent ASD participants and 18 typically developing controls performed an engaging visual paradigm with embedded visual grating, to robustly induce alpha $(8-13 \mathrm{~Hz})$ and gamma $(40-80 \mathrm{~Hz})$ oscillations. We characterised changes in power and connectivity between visual areas V1 and V4: two regions which show strong hierarchical connectivity (Bastos et al., 2015; Michalareas, Vezoli, Van Pelt, et al., 2016). Additionally, we quantified local alpha-gamma PAC for area V1 (Özkurt \& Schnitzler, 2011; Seymour, Rippon, \& Kessler, 2017; Tort, Komorowski, Eichenbaum, \& Kopell, 2010a).

\subsection{Materials and Methods}

\subsubsection{Participants}

Data were collected from 18 participants diagnosed with autism spectrum disorder and 18 age-matched typically developing controls, see Table 1.1 and Section 1.7.

\subsubsection{Experimental Procedures}

All experimental procedures complied with the Declaration of Helsinki and were approved by the Aston University, Department of Life \& Health Sciences ethics committee. Written consent was obtained from participants aged 18 or over, or from a parent/guardian for participants aged under 18.

\subsubsection{Behavioural Assessments}

General non-verbal intelligence was assessed using the Raven's Matrices Task (Raven \& Court, 1998). The severity of autistic traits was assessed using the Autism Quotient, $A Q$, and sensory traits using the Glasgow Sensory Questionnaire, GSQ, (Robertson \& Simmons, 2013). Both AQ and GSQ scores were higher in the ASD group (Table 1). Participants also completed the Mind in the Eyes test (Baron-Cohen, Wheelwright, Hill, Raste, \& Plumb, 2001), however, there were no group differences in scores from this assessment, see Table 3.1. The Mind in the Eyes test has been recently criticised for measuring emotion recognition rather than an autism-specific deficit in mental state 
attribution (Oakley, Brewer, Bird, \& Catmur, 2016), and therefore these scores were not used to investigate correlations between brain patterns and questionnaire measures.

\subsubsection{Paradigm}

Whilst undergoing MEG, participants performed an engaging sensory task, identical to that used in Chapter 2. Prior to MEG acquisition, the nature of the task was fully explained to participants and several practice trials were performed. Accuracy rates were above $95 \%$ for all participants indicating that the task was engaging and successfully understood.

\subsubsection{MEG Acquisition}

MEG data were acquired using a 306-channel Neuromag MEG scanner (Vectorview, Elekta, Finland) made up of 102 triplets of two orthogonal planar gradiometers and one magnetometer. All recordings were performed inside a magnetically shielded room at a sampling rate of $1000 \mathrm{~Hz}$. Five head position indicator (HPI) coils were applied for continuous head position tracking, and visualised post-acquisition using an in-house Matlab script. For MEG-MRI coregistration purposes three fiducial points, the locations of the HPI coils and 300-500 points from the head surface were acquired using the integrated Polhemus Fastrak digitizer. Visual stimuli were presented on a screen located $86 \mathrm{~cm}$ from participants (resulting in 2 cycles/degree for the visual grating), and auditory feedback through MEG-compatible earphones.

\subsubsection{Structural MRI}

A structural T1 brain scan was acquired for source reconstruction using a Siemens MAGNETOM Trio 3T scanner with a 32-channel head coil $(T E=2.18 \mathrm{~ms}$, TR=2300ms, $\mathrm{TI}=1100 \mathrm{~ms}$, flip angle $=9^{\circ}, 192$ or 208 slices depending on head size, voxel-size $=$ $0.8 \times 0.8 \times 0.8 \mathrm{~cm})$.

\subsubsection{MEG-MRI Coregistration and Cortical Mesh Construction}

MEG data were co-registered with participants MRI structural scan by matching the digitised head-shape data with surface data from the structural scan (Jenkinson \& Smith, 2001). Two control participants did not complete a T1 structural MRI and therefore a pseudo-MRI was used (see Gohel, Lim, Kim, Kwon, \& Kim (2017) for full procedure). The aligned MRI-MEG images were used to create a forward model based on a single-shell description of the inner surface of the skull (Nolte, 2003), using the segmentation function in SPM8 (Litvak et al., 2011). The cortical mantle was then extracted to create a cortical mesh, using Freesurfer v5.3 (Fischl, 2012), and registered to a standard fs_LR mesh, 
based on the Conte69 brain (Van Essen 2012), using an interpolation algorithm from the Human Connectome Project (Van Essen et al., 2012; instructions here:

https://goo.gl/3HYA3L). Finally, the mesh was downsampled to 4002 vertices per hemisphere.

\subsubsection{MEG Pre-Processing}

MEG data were pre-processed using Maxfilter (temporal signal space separation, .9 correlation), which supresses external sources of noise from outside the head (Taulu \& Simola, 2006). Further pre-processing steps were performed in Matlab 2014b using the Fieldtrip toolbox v20161024 (Oostenveld, Fries, Maris, \& Schoffelen, 2010). Firstly, for each participant the entire recording was band-pass filtered between $0.5-250 \mathrm{~Hz}$ (Butterworth filter, low-pass order 4, high-pass order 3) and band-stop filtered (49.5$50.5 \mathrm{~Hz} ; 99.5-100.5 \mathrm{~Hz}$ ) to remove residual $50 \mathrm{~Hz}$ power-line contamination and its harmonics. Data were epoched into segments of $4 \mathrm{~s}(1.5 \mathrm{~s}$ pre, $1.5 \mathrm{~s}$ post stimulus onset, with $0.5 \mathrm{~s}$ of padding either side) and each trial was demeaned and detrended. Trials containing artefacts (SQUID jumps, eye-blinks, head movement, muscle) were removed if the trial-by-channel (magnetomer) variance exceeded $8 \times 10^{-23}$, resulting in an average of 3.1 trials per participant. Four MEG channels containing large amounts of nonphysiological noise were removed from all analyses.

\subsubsection{Source-Level Gamma and Alpha Power}

Source analysis was conducted using a linearly constrained minimum variance beamformer (Van Veen et al., 1997), which applies a spatial filter to the MEG data at each vertex of the cortical mesh. Due to differences in noise between sensor-types, covariance matrix terms resulting from multiplying magnetomer and gradiometer data were removed. Beamformer weights were calculated by combining this covariance matrix with leadfield information, with data pooled across baseline and grating periods. Following tSSS, sensor-level data had a rank 64 or below, and therefore a regularisation parameter of lambda $5 \%$ was applied. Data were band-pass filtered between $40-80 \mathrm{~Hz}$ (gamma) and 8$13 \mathrm{~Hz}$ (alpha), and source analysis was performed separately. To capture induced rather than evoked visual power, a period of $0.3-1.5 \mathrm{~s}$ following stimulus onset was compared with a $1.2 \mathrm{~s}$ baseline period (1.5-0.3s before grating onset).

\subsubsection{ROI definition}


In order to quantify directed connectivity within the visual system, we selected two regions of interest (ROI): visual area 1 (V1) and visual area 4 (V4). Both regions show changes in oscillatory power following presentation of the visual grating (Figure 3.1E-F) and demonstrate reliable patterns of hierarchical connectivity: V1-to V4 connectivity is feedforward; whereas V4-to-V1 connectivity is feedback (Bastos et al., 2015; Bastos et al., 2015; Felleman \& Van, 1991; Michalareas, Vezoli, Van Pelt, et al., 2016). The ROls were defined using a multi-modal parcellation from the Human Connectome Project, which combined retinotopic mapping, T1/T2 structural MRI and diffusion-weighted MRI to accurately define the boundaries between cortical areas (Glasser et al., 2016; Figure 3.1D). 12 vertices from posterior $\mathrm{V} 1$ were excluded to ensure clear anatomical separation between area V1 and V4. To obtain a single spatial filter for each ROI, we performed a principle components analysis (PCA) on the concatenated filters encompassing $\mathrm{V} 1$ and V4, multiplied by the sensor-level covariance matrix, and extracted the first component. The sensor-level data was then multiplied by this spatial filter to obtain bilateral V1 and V4 specific "virtual electrodes". Finally, the change in oscillatory power between grating and baseline periods was calculated using multi-taper approach (Hoogenboom, Schoffelen, Oostenveld, Parkes, \& Fries, 2006) from $1-140 \mathrm{~Hz}$, using a 0.5 s time window, sliding in steps of $0.02 \mathrm{~s}$ and $\pm 8 \mathrm{~Hz}$ frequency smoothing.

\subsubsection{V1-V4 Directed Connectivity}

To quantify the directed functional connectivity between V1 and V4, we employed Granger causality (GC) - a statistical technique which measures the extent to which one time series can predict another (Granger, 1969). Following the approaches outlined by Bastos et al., (2015) and Michalareas, Vezoli, Van Pelt, et al., (2016), we employed a spectrally resolved non-parametric version of GC as implemented in the Fieldtrip toolbox (Dhamala, Rangarajan, \& Ding, 2008; Oostenveld, Fries, Maris, \& Schoffelen, 2011). Data from bilateral V1 and V4 ROIs (0.3-0.1.5s post stimulus onset) were split into $0.4 \mathrm{~s}$ epochs to enhance the accuracy of results, fouier transformed (Hanning taper; $2 \mathrm{~Hz}$ spectral smoothing), and entered into a non-parametric spectral matrix factorisation procedure. GC was then estimated between $1-140 \mathrm{~Hz}$ for each ROI pair and collapsed across hemispheres post-hoc. V1-to-V4 GC values were classified as 'feedforward', and V4-to-V1 as 'feedback' (Bastos, Vezoli, et al., 2015; Michalareas, Vezoli, Van Pelt, et al., 2016). Scrambled time-series with the same spectral properties as V1/V4 were created for comparison, modelled using the first autoregressive coefficient (Colclough et al., 2015), and the above steps were repeated. 
Asymmetries in GC values between V1 and V4 were quantified using the Directed Asymmetry Index (DAI) using the following formula from Bastos et al., (2015) and Michalareas, Vezoli, Van Pelt, et al., (2016).

$$
D A I=\frac{G C(V 1 \rightarrow V 4)-G C(V 4 \rightarrow V 1)}{G C(V 1 \rightarrow V 4)+G C(V 4 \rightarrow V 1)}
$$

This results in normalised values between -1 and 1 for every frequency of the granger spectrum $(1-140 \mathrm{~Hz})$, with values above 0 indicating feedforward granger-causal influence and values below 0 indicating feedback influence. DAI values from ASD and typically developing groups were then statistically compared.

\subsubsection{V1 Phase-Amplitude Coupling (PAC)}

Time-courses from bilateral V1 were examined for changes in alpha-gamma phase amplitude coupling (PAC). Detailed discussion around PAC computation and methodological issues can be found in Chapter 2 and Seymour, Rippon, \& Kessler (2017). Briefly, we calculated PAC values between phases $7-13 \mathrm{~Hz}$ (in $1 \mathrm{~Hz}$ steps) and amplitudes $34-100 \mathrm{~Hz}$ (in $2 \mathrm{~Hz}$ steps), for the time-period $0.3-1.5$ s following grating presentation. PAC values were corrected using 1.2 of data from the baseline period. This resulted in a $33^{\star} 7$ amplitude-phase comodulogram for ASD and control groups, which were statistically compared using a cluster-based permutation test (Maris \& Oostenveld, 2007).

To calculate PAC values, we used the mean vector length (MVL) approach from Özkurt \& Schnitzler (2011) and the phase-locking value (PLV) approach from Cohen (2008), based on the results of Chapter 2. Code used for PAC computation can be found openly at https://github.com/neurofractal/sensory_PAC.

\subsubsection{Statistical Analysis}

Statistical analysis was performed using cluster-based permutation tests (Maris and Oostenveld, 2007), which consist of two parts: first an independent-samples t-test is performed, and values exceeding an uncorrected $5 \%$ significance threshold are grouped into clusters. The maximum t-value within each cluster is carried forward. Second, a null distribution is obtained by randomising the condition label (e.g. ASD/control) 1000 times and calculating the largest cluster-level t-value for each permutation. The maximum tvalue within each original cluster is then compared against this null distribution, and the null hypothesis is rejected if the test statistic exceeds a threshold of $p<.05$. 


\subsection{Results}

\subsubsection{Gamma and Alpha Power}

The change in oscillatory power following presentation of the visual grating was calculated on a cortical mesh for the alpha $(8-13 \mathrm{~Hz})$ and gamma $(40-80 \mathrm{~Hz})$ bands. For both ASD and control groups there was a statistically significant increase in gamma-band power from $40-80 \mathrm{~Hz}$ (Figure 3.1B) and a decrease in alpha-band power from $8-13 \mathrm{~Hz}$ (Figure 3.1C), localised to the ventral occipital cortex. This replicates many previous MEG/EEG studies using visual grating stimuli (Hoogenboom et al., 2006; Michalareas, Vezoli, Van Pelt, et al., 2016; Muthukumaraswamy, 2013). Interestingly, there were no significant differences in either gamma or alpha power between groups ( $p>.33$ gamma and $p>.14$ alpha, Figure 3.2).

Two regions of interest (ROI) were defined in V1 and V4 (Figure 3.1D). Changes in oscillatory power from V1 (Figure 3.1E) and V4 (Figure 3.1F) showed characteristic increases in gamma-band power $(40-80 \mathrm{~Hz})$ and decreases in alpha/beta power $(8-20 \mathrm{~Hz})$. Between groups, there were minor differences between the power spectrums, including a larger alpha/beta peak for the ASD group (Fig 2E, 2F, purple line), however none of these differences reached significance (both $p>05$ ). 

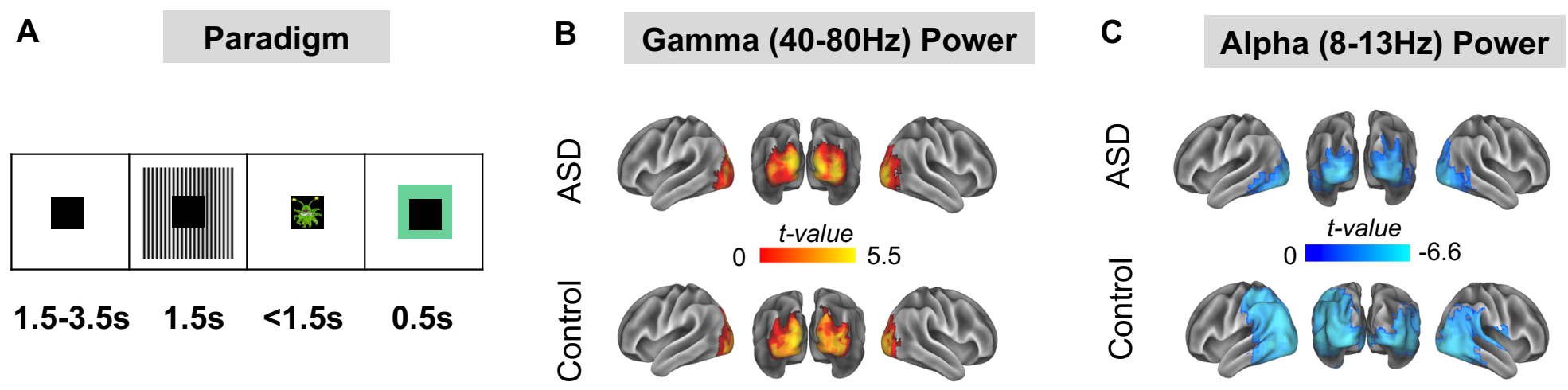

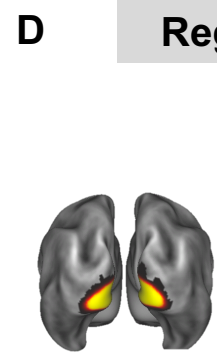

V1

\section{Regions of Interest}

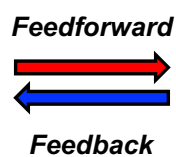

Feedback

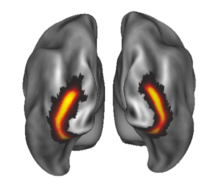

V4

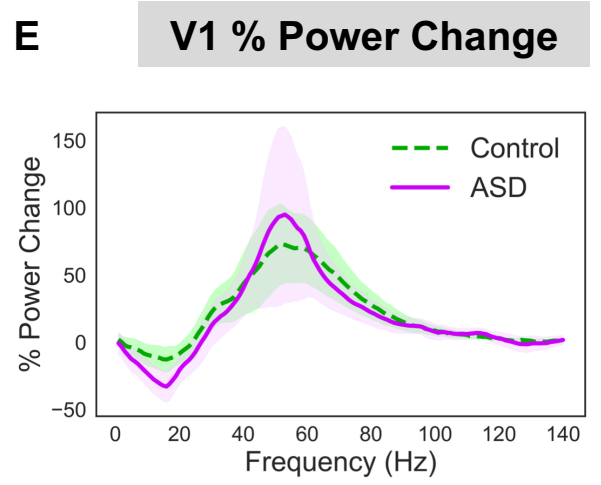

\section{F $\quad$ V4 \% Power Change}

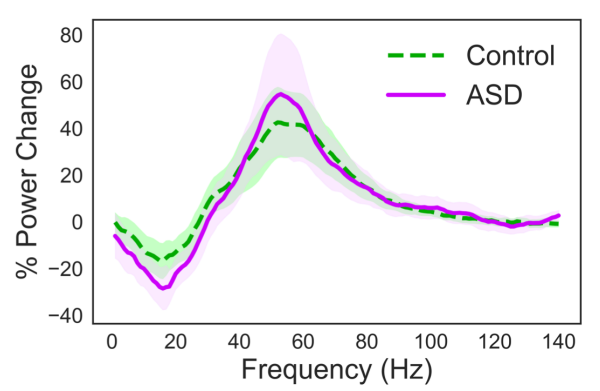

Figure 3.1. (A) Trial progression for the engaging visual task with embedded grating, see Section 3.3.4 for more information. The alien/astronaut stimuli were to maintain attention and do not form part of the analysis. (B-C) The change in oscillatory power between grating and baseline periods was localised on a cortical mesh, and masked to show only statistically significant ( $p<.05$, corrected) stimulus induced increases in gamma (40-80Hz) and deceases in alpha (8-13Hz) power. There were no statistically significant differences in gamma or alpha power between groups. (D) Regions of interest in V1 and V4 were defined using HCP-MMP atlas. (E-F) The change in power between grating and baseline periods was calculated for V1 and V4 from 1-140Hz. Results show characteristic reductions in alpha/beta power and increases in gamma-band power (40-80Hz) for V1 and V4. There were no statistically significant differences in power between groups. The shaded area around each curve indicates $95 \%$ confidence intervals. 


\section{Control>ASD Power}

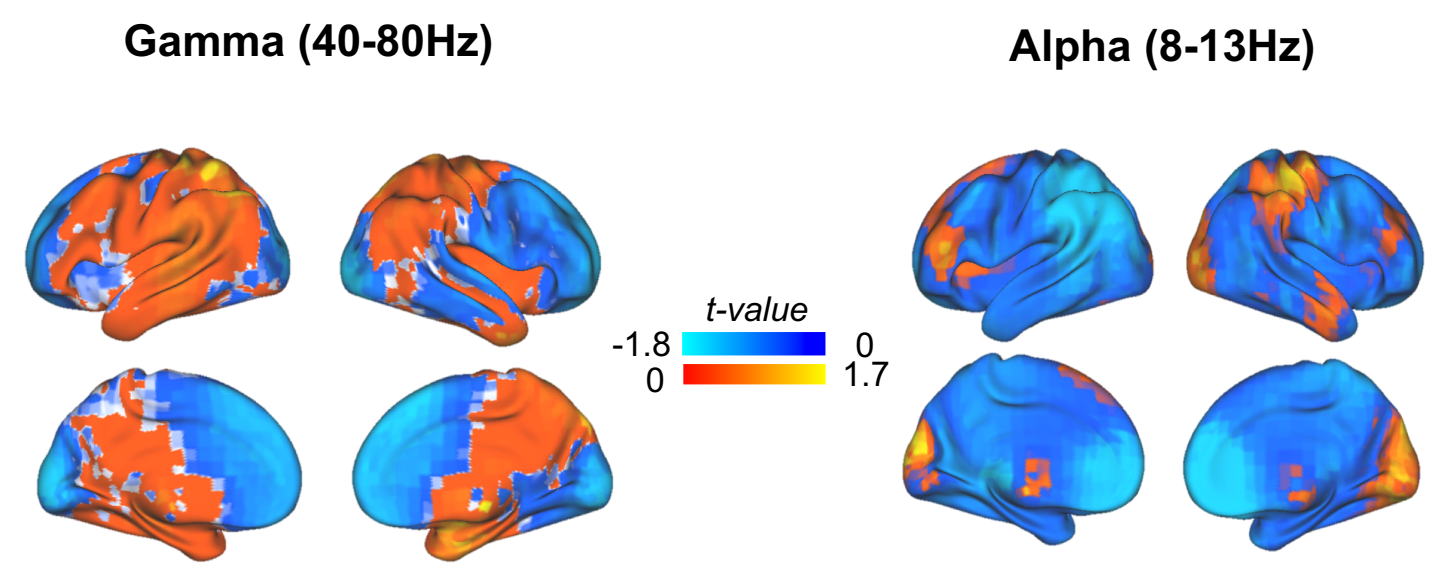

Figure 3.2. Statistical comparison of control>ASD source-space oscillatory power for gamma $(40-80 \mathrm{~Hz})$ and alpha $(8-13 \mathrm{~Hz})$. There were no significant differences in either alpha or gamma power between groups (all clusters $p>.05$, corrected for multiple comparisons).

\subsubsection{Feedforward / Feedback Connectivity}

The directed functional connectivity between V1 and V4 was quantified using Granger causality (GC). Across both groups, all reported increases in bidirectional V1-V4 GC were significantly greater than for scrambled data $(p<.05$, see Figure 3.3$)$. For the control group (Figure 3.4A), V1-to-V4 (henceforth termed feedforward) connectivity showed a prominent increase from $40-80 \mathrm{~Hz}$ in the gamma band. In contrast, V4-to-V1 (henceforth termed feedback) connectivity showed a prominent increase from $8-13 \mathrm{~Hz}$ in the alpha band (Figure 3.4A). This dissociation between feedforward gamma and feedback alpha, replicates findings from macaque visual cortex using ECOG (Bastos et al., 2015; Kerkoerle et al., 2014) and human visual cortex using MEG (Michalareas, Vezoli, Van Pelt, et al., 2016). The feedforward gamma-band peak $(40-80 \mathrm{~Hz})$ was also evident in the ASD granger spectra (Figure 3.4B, red line). However, there was a reduction in the alphaband feedback peak in the ASD group compared with controls (Figure 3.4B, blue line). 


\section{V1-V4 GC Scrambled Data vs Intact Data}

Control

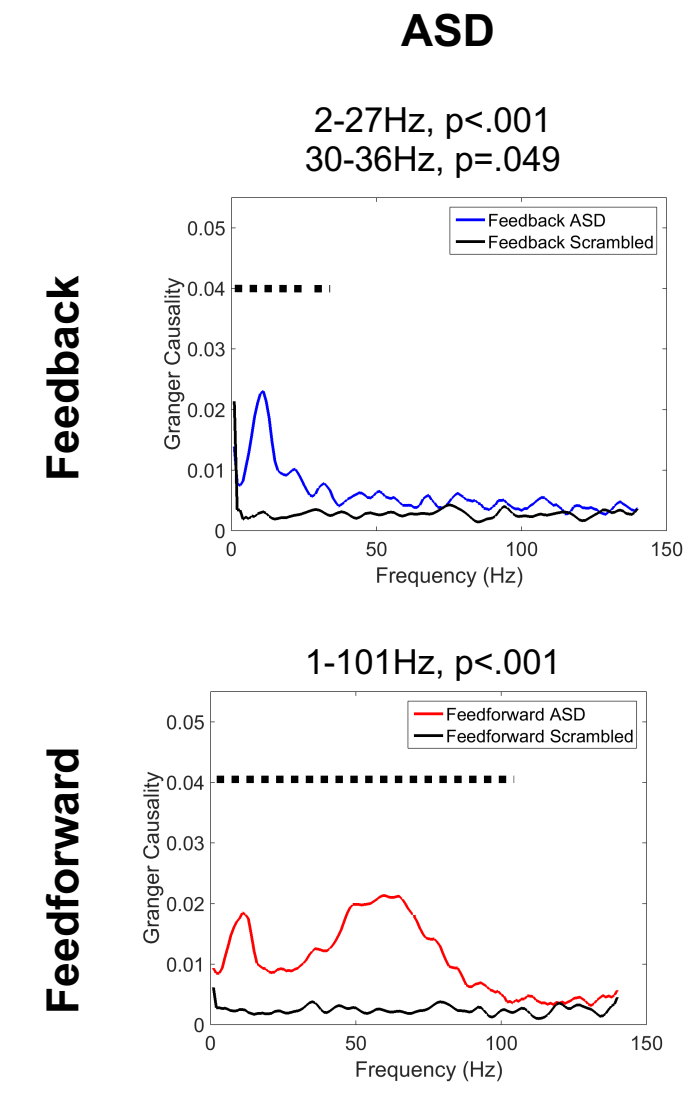

$2-27 \mathrm{~Hz}, \mathrm{p}<.001$

$30-36 \mathrm{~Hz}, p=.049$

$1-101 \mathrm{~Hz}, \mathrm{p}<.001$
$2-27 \mathrm{~Hz}, \mathrm{p}<.001$

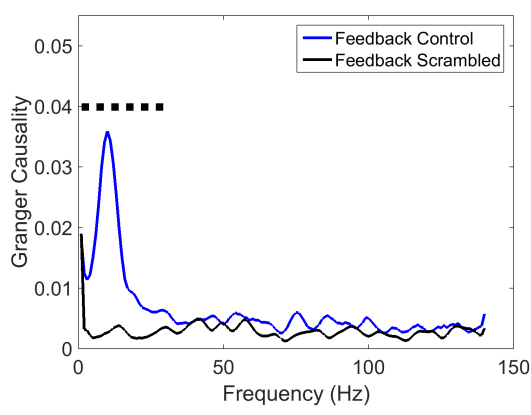

$1-83 \mathrm{~Hz}, p<.001$

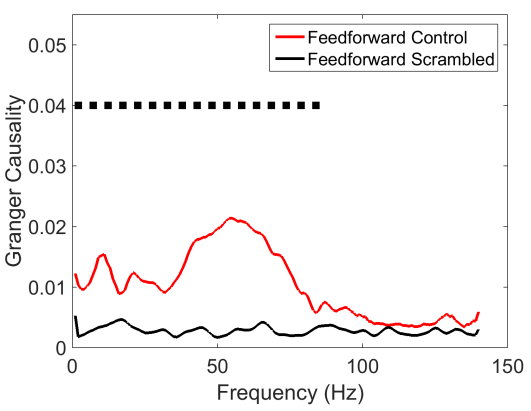

Figure 3.3. V1-V4 feedforward and V4-V1 Granger Causality (GC) values were statistically compared with GC values computed using scrambled V1/V4 data with same spectral properties as the intact data. On each sub-Figure 3.the black dotted line signifies intact $G C$ values significantly greater than scrambled $G C$ values $(p<.05)$. The exact frequency range and $p$-values are listed at the top of each plot.

To quantify asymmetries in feedforward and feedback connectivity between groups, we calculated the directed asymmetry index (DAI, see Section 3.3.11). The control group displayed a characteristic feedback peak from $1-20 \mathrm{~Hz}$ (indicated by negative DAl values) and feedforward peak (indicated by positive DAI values) from $40-80 \mathrm{~Hz}$. By statistically comparing DAI between groups and it was found that values from $8-14 \mathrm{~Hz}$ were significantly lower $(p=.032)$ for the control group than the ASD group. All other frequencies, including gamma $(40-80 \mathrm{~Hz})$ showed similar DAl values between groups. This suggests that the ASD group display reduced feedback connectivity within the visual system, mediated by low-frequency oscillations, predominantly in the alpha-band (8$14 \mathrm{~Hz}$ ), but typical feedforward connectivity mediated by oscillations in the gamma-band $(40-80 \mathrm{~Hz})$. 
Interestingly there was no feedforward granger-causality peak in the theta-band $(4-8 \mathrm{~Hz})$ for either the control or ASD group, as previously reported using invasive ECoG (Spyropoulos, Bosman, \& Fries, 2018). This could be due to lower sensitivity of MEG recordings compared with ECoG, also see Michalareas, Vezoli, Van Pelt, et al., (2016), as well as the use of a centrally-masked visual grating (Figure 3.1A).

\subsubsection{Alpha-Gamma Phase Amplitude (PAC) in V1}

Activity from visual area V1 was examined for changes in PAC, between alpha-band phase and gamma-band amplitude. Two algorithms were used to quantify PAC: the Mean-Vector Length approach from Özkurt \& Schnitzler (2011), MVL-Ozkurt, and the Kullbeck-Leiber approach from Tort et al., (2010a), KL-Tort. Frequency-by-frequency comodulograms showed PAC increases in the control group, peaking at $8-10 \mathrm{~Hz}$ phase frequencies and $50-70 \mathrm{~Hz}$ amplitude frequencies (Figure 3.5A,B). These results replicate the results from Chapter 2 using the same paradigm. The comodulograms for the ASD group display lower PAC values, with no clear positive peak (Figure 3.5B,C). Comparing control vs. ASD groups using the MVL-Ozkurt approach, there was a single positive cluster of greater PAC between $8-9 \mathrm{~Hz}$ phase and 52-74Hz amplitude, $\mathrm{p}=.029$, (Figure 3.5E). Similar results were reported for the KL-Tort approach (Figure 3.5F). This suggests that the coupling between alpha and gamma oscillations in primary visual cortex during perception is reduced in autism. The similarity in PAC comodulograms between MVLOzkurt and KL-Tort approaches, indicates that the results generalise across both PAC metrics. 


\section{V1-V4 Feedforward/Feedback Connectivity}

A

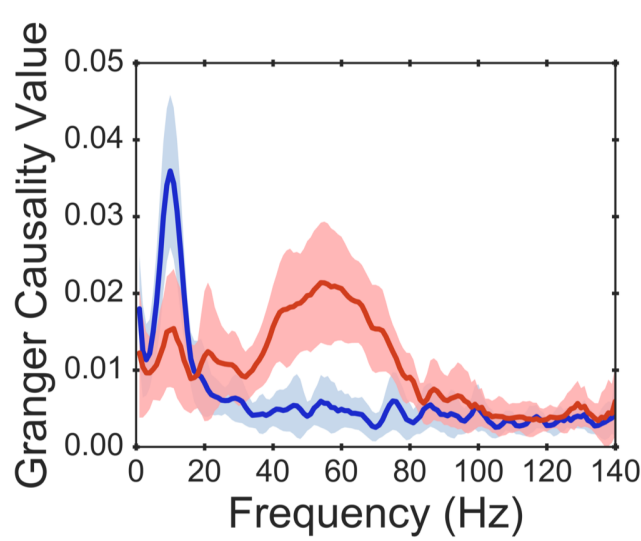

- Feedback
Control

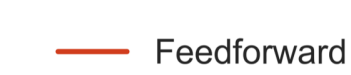

B

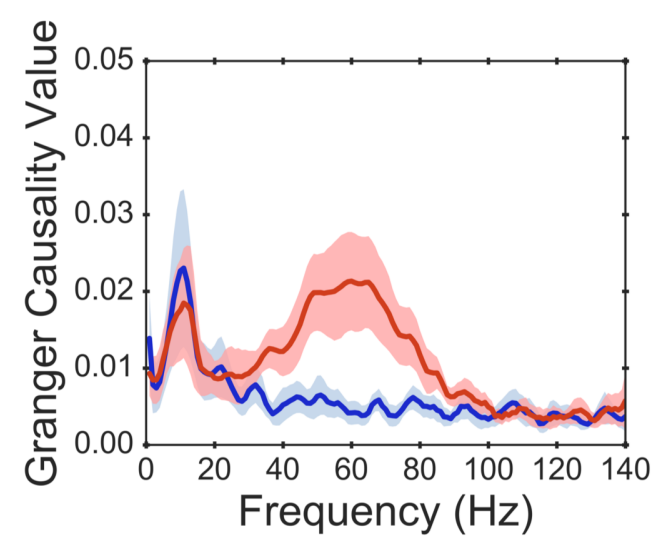

Feedback — Feedforward
C Directed Asymmetry Index

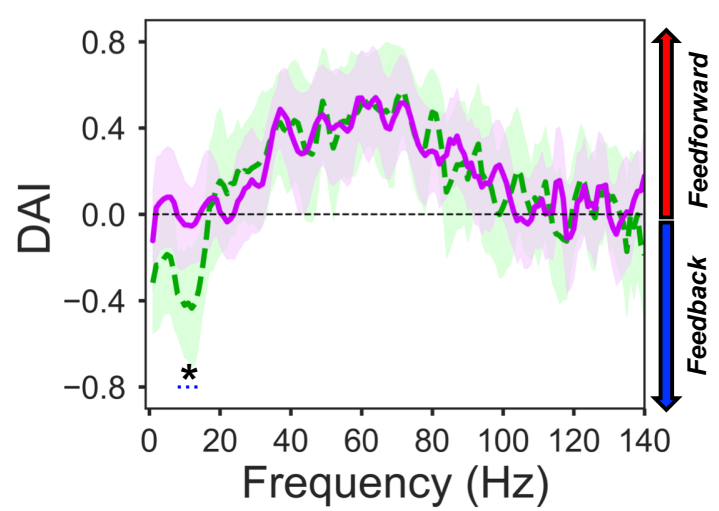

--- Control $\longrightarrow$ ASD

Figure 3.4. V1-V4 Feedforward/Feedback Connectivity. (A) For the control group there was a peak in granger causality (GC) values, in the gammaband (40-80Hz, red line) for V1-to-V4 feedforward connectivity, and a peak in GC values in the alpha band (8-14Hz, blue line) for V4-to-V1 feedback connectivity. (B) For the ASD group there was also a peak in GC values in the gamma-band for V1-to-V4 feedforward connectivity, however there was smaller peak in GC in the alpha-band for V4-to-V1 feedback connectivity. (C) The difference between feedforward and feedback connectivity was quantified as the directed asymmetry index (DAl, see Material and Methods). The difference in DAI between control (dashed, green line) and ASD (solid, purple line) was significant ( $p=.036)$, with lower DAl values $(p=.036)$ between $8-14 \mathrm{~Hz}$ for the control group, suggesting reduced V4-to-V1 feedback connectivity in autism. The shaded area around each GC line indicates $95 \%$ confidence intervals. 


\section{V1 Alpha-Gamma Phase Amplitude Coupling (PAC)}
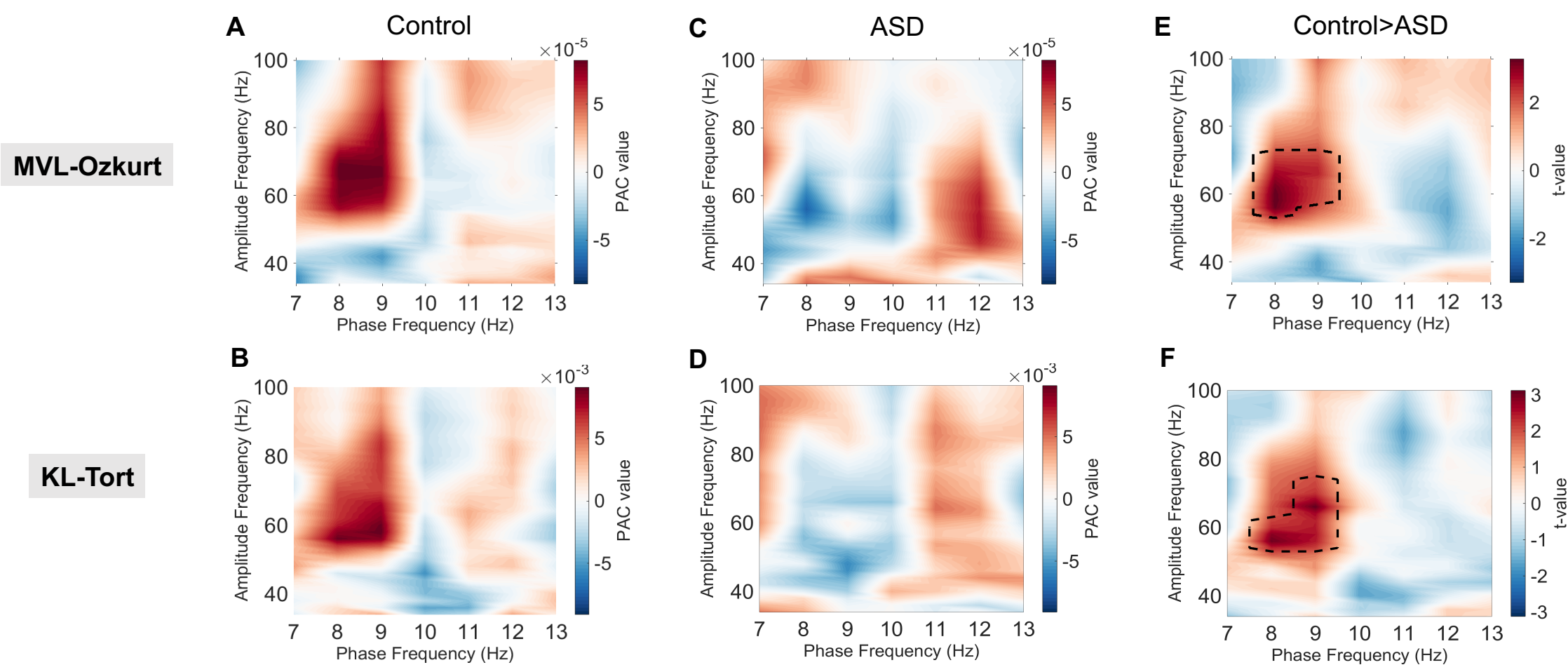

Figure 3.5. V1 alpha-gamma PAC. The top panel shows comodulograms using the MVL-Ozkurt approach, the bottom panel using the KL-Tort approach. $(\boldsymbol{A}, \boldsymbol{B})$ The control group showed increased alpha-gamma PAC, with a peak between $50-80 \mathrm{~Hz}$ amplitude and 7-9Hz phase. (C,D) The ASD group showed less prominent increases in PAC with a much smaller peak from 40-70Hz amplitude and 11-13Hz phase. (E) Statistical comparison of control>ASD (MVL-Ozkurt approach) indicated one positive cluster of increased PAC for the control group ( $p=.029)$ from 54-72Hz amplitude and 8$9 \mathrm{~Hz}$ phase. (F) Similar results were found using the KL-Tort approach, with one positive cluster of increased $P A C(p=.037)$ from $54-74 \mathrm{~Hz}$ amplitude and $8-9 \mathrm{~Hz}$ phase. 


\subsubsection{Feedback - PAC Correlation}

As described in Section 3.2, if top down connectivity influences local processing then a relationship between feedback alpha and local alpha-gamma PAC should be observed. To investigate this across groups, we ran an exploratory correlation analysis between alpha-band feedback connectivity (DAl for alpha) and V1 PAC. There was a negative correlation between 9Hz DAl and 8Hz PAC across both groups (Figure 3.6, Pearson's $r=$ $-.35, p=.034$, uncorrected for multiple comparisons across the cross-correlation matrix) suggesting that increased V4-to-V1 feedback connectivity is related to greater local PAC in $\mathrm{V} 1$.

\section{DAI + PAC Correlation}

A

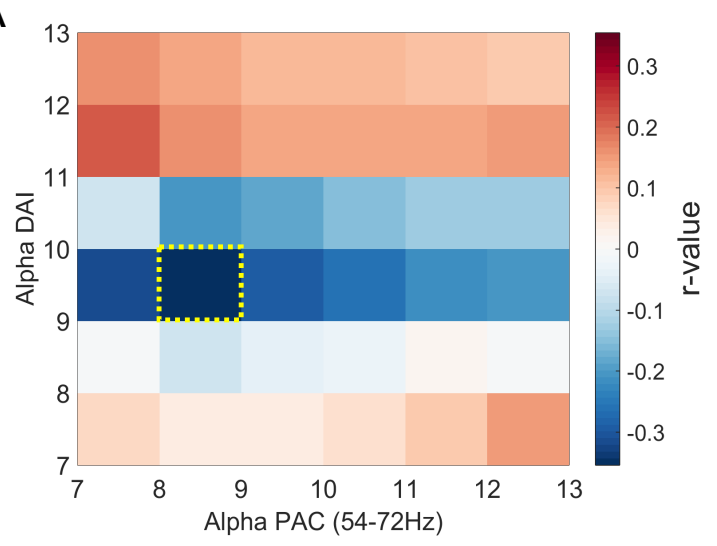

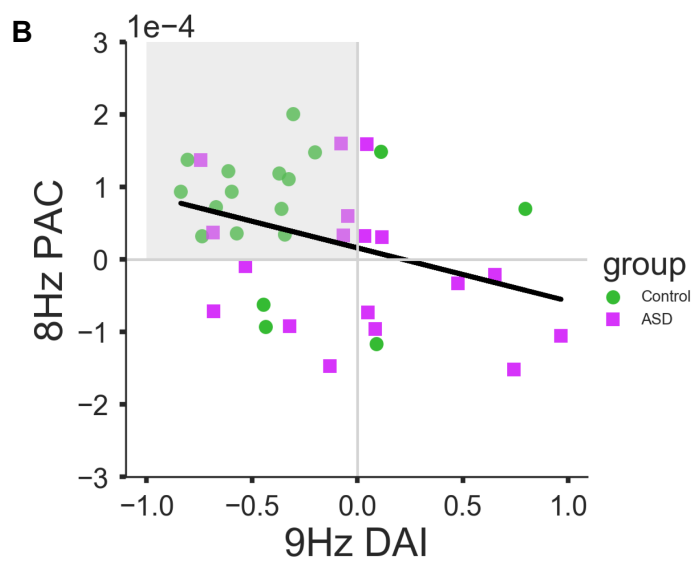

Figure 3.6. (A) To investigate the correlation between feedback connectivity and PAC, a cross correlation matrix was calculated in $1 \mathrm{~Hz}$ steps between ASD and control participant's alpha PAC, averaged between $54-72 \mathrm{~Hz}$, and $7-13 \mathrm{~Hz}$ directed asymmetry index (DAl). This produced a negative correlation peak, shown with yellow box, at $8 \mathrm{~Hz}$ PAC, 9Hz DAl. (B) The correlation between $8 \mathrm{~Hz} P A C, 9 H z$ DAl is negative across both groups (Pearson's $r=-.35, p=.034$ ). Please note that most of the control participants (green circles) are located in the top right quadrant (highlighted in grey), whereas the ASD participants (purple squares) display a more variable pattern.

\subsubsection{Connectivity - Behaviour Correlation}

Behavioural ASD data from the Autism Quotient (AQ) and Glasgow Sensory Questionnaires (GSQ) were correlated with alpha-band DAI (averaged over $8-13 \mathrm{~Hz}$ ) and alpha-gamma PAC values (averaged over $54-72 \mathrm{~Hz}$ amplitude and $8-9 \mathrm{~Hz}$ phase), identified in the previous group comparisons (see Figure 3.4C and Figure 3.5E). There was a significant positive correlation between AQ score and alpha DAI (Figure 3.7B, Pearson's $r=.526, p=.025$ ) suggesting that increased feedback connectivity between V4 
and V1 (negative DAI values) is related to lower levels of autistic traits (lower AQ scores). There were no other significant correlations for the GSQ or PAC. Due to the lower variability in control $A Q$ data (minimum $=6$; $\max =21 ; S D=5.63$ ) and $G S Q$ data ( minimum $=29$; maximum $=50 ; S D=7.14$ ), it would be statistically inappropriate to perform correlations with connectivity values for this group. Therefore only ASD connectivity - behaviour correlations are reported.

\section{Correlations with $A Q$ and $G S Q$}
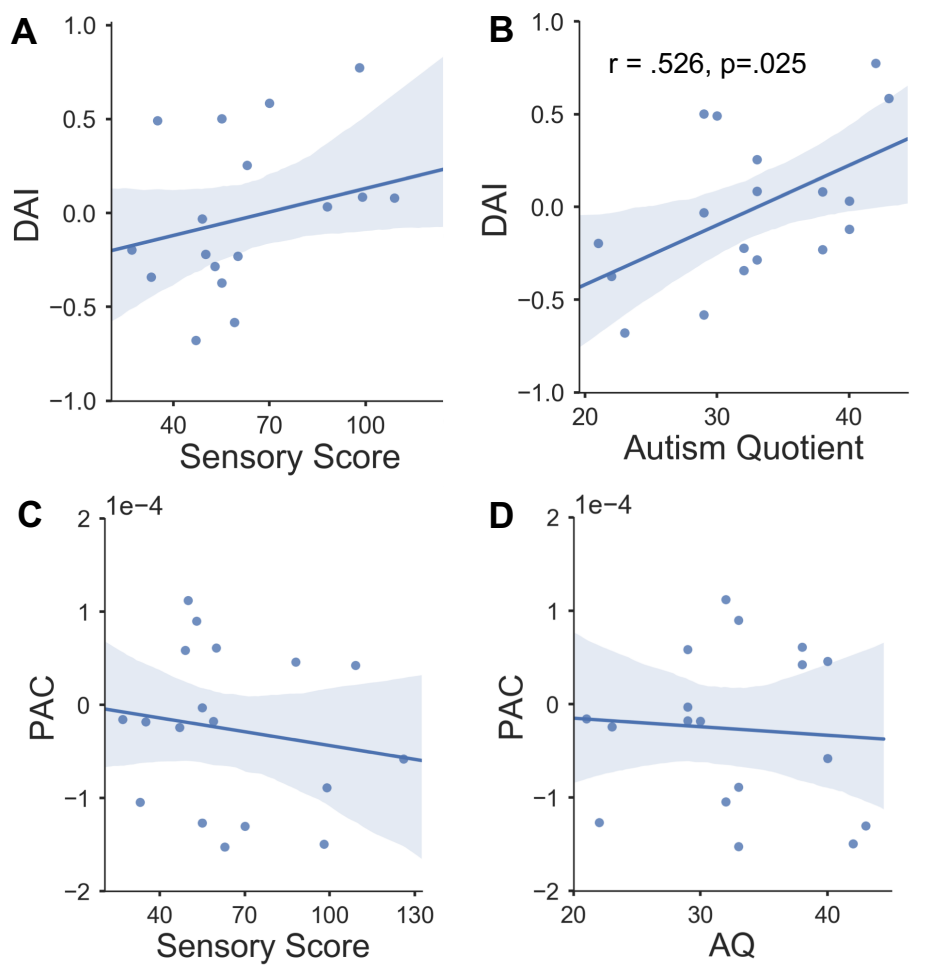

Figure 3.7. For the ASD group, the correlation between alpha-band DAI (A-B), alphagamma PAC (C-D) and Autism Quotient $(B, D)$, Glasgow Sensory Score $(A, C)$ was plotted with regression line (95\% confidence interval indicated by shaded region). (B) There was a positive correlation between $D A I$ and $A Q$ score.

\subsection{Discussion}

This study examined the oscillation-based functional connectivity within the visual system of autistic adolescents and typically developing age-matched controls. Confirming our hypotheses (Kessler et al., 2016), we found that although there were no group differences in alpha or gamma-band power, there was a reduction in alpha-band $(8-14 \mathrm{~Hz})$ feedback connectivity from V4-to-V1 in the ASD group. This reduction in feedback connectivity was positively correlated with autistic traits (less feedback connectivity was related to higher autistic traits). Additionally, there was a reduction in the coupling between alpha and 
gamma oscillations in V1, as measured via PAC, suggesting a dysregulation of local connectivity in autism.

\subsubsection{Feedback / Feedforward Connectivity}

The reduction in feedback connectivity is consistent with previous studies showing a reduction in global connectivity during cognitive tasks and at rest, measured using MEG and fMRI (Hughes, 2007; Khan et al., 2013; Kitzbichler et al., 2015). In this instance, a simple visual paradigm showed that the reduction in feedback connectivity was specific to oscillations in the alpha band from $8-14 \mathrm{~Hz}$ (Figure 3.4). Alpha-band oscillations underlie the functional inhibition and disengagement of task-irrelevant brain regions, promoting information flow through precise timing of neural activity (Klimesch, 2012). Posterior alpha-band activity is also a mechanism for top-down modulation of perceptual processes, linked with spatial attention and phase-locking with wider frontoparietal control networks (Capotosto, Babiloni, Romani, \& Corbetta, 2009; Palva \& Palva, 2011). Our data suggest that in autism, whilst overall alpha power appears to be unaffected, the feedback flow of information from higher to lower visual regions is reduced. An inability to implement topdown modulation of bottom-up visual information, may result in the atypical sensory processes reported by those on the autistic spectrum and contribute to the severity of autism symptoms more generally (Kessler et al., 2016; Simon \& Wallace, 2016). In support of this, we found a correlation between the reduction in feedback connectivity and $A Q$ score.

Interestingly, we did not find an increase in connectivity from V1-to-V4 for the ASD group mediated by oscillations in the gamma-band, suggesting typical feedforward flow of visual information in ASD. Whilst Khan et al., (2015) reported increased feedforward connectivity in autism, that study focussed on somatosensory rather than visual processing with a younger group of adolescent participants. In any case, we hypothesise that where perception can be achieved via feedforward processes (VanRullen \& Koch, 2003), autistic participants will perform on par or even outperform their typically developing peers (Mottron, Dawson, Soulieres, Hubert, \& Burack, 2006). For example, autistic participants perform faster during visual search tasks, potentially because feedforward parallel visual feature processing is likely to yield better results compared to neurotypical processing, where irrelevant, distracting feedback information interferes with feedforward feature processing (Jobs, Falck-Ytter, \& Bölte, 2018; Kaldy, Giserman, Carter, \& Blaser, 2016). 
The reported differences in feedback, but not feedforward connectivity suggest that where possible, studies examining global connectivity in autism should consider the direction of functional connectivity under investigation. Electrophysiological techniques are well suited to capture transient changes in directed functional connectivity and the oscillatory processes supporting this (Fries, 2015). In future, layer-specific functional MRI could also be used to map laminar differences in feedforward versus feedback processes in ASD (Kok, Bains, van Mourik, Norris, \& de Lange, 2016; Muckli et al., 2015).

\subsubsection{PAC}

At the local level, we found a reduction in alpha-gamma PAC within primary visual cortex for the ASD group (Figure 3.5). Reduced alpha-gamma PAC in autism has previously been reported during emotional face perception in the fusiform gyrus (Khan et al., 2013), and during rest (Berman et al., 2015). This suggests that increases in local gamma power, driven by visual input, are decoupled from wider patterns of top-down alpha-band connectivity in autism. These findings also link with neurophysiological models of ASD at the cellular level. As PAC relies heavily on local inhibitory populations of neurons (Onslow, Jones, \& Bogacz, 2014), a reduction in PAC is consistent with histological findings showing underdeveloped inhibitory interneurons (Casanova, Buxhoeveden, \& Gomez, 2003), and an E-I imbalance in autism (Rubenstein \& Merzenich, 2003). Where local inhibitory processes are affected, this would manifest as high-frequency 'noisy' activity in the brain, common in ASD (Casanova, Buxhoeveden, \& Gomez, 2003), and reduced signal-to-noise (Rubenstein \& Merzenich, 2003). Dysregulated local activity could also have concomitant effects on establishing patterns of global connectivity in autism (Voytek \& Knight, 2015). Indeed, in this study there was a correlation between feedback connectivity and the strength of PAC across both ASD and control groups (Figure 3.6), similar to findings of Khan et al., (2013). However, we did not find a relationship between $A Q$ or GSQ and PAC in the autistic group (Figure 3.7D). In contrast, Mamashli et al., (2018) reported a correlation between Autism Diagnostic Observation Schedule (ADOS) social score and local PAC in a 14-21 year old autistic sample, suggesting that PAC may be related to clinical features of autism rather than general autistic traits (see 3.5.4 Limitations).

It is important to note that the group differences in oscillatory connectivity arose despite similar changes in gamma and alpha power between ASD and control groups (Figure 3.1). Findings in relation to differences in gamma/alpha power and connectivity in ASD, are very mixed, see Chapter 1 Section 1.4. This may reflect the heterogeneity of autism as a disorder, as well as the mixture of hypo and hyper-sensitivities reported by autistic 
people (Hazen, Stornelli, O’Rourke, Koesterer, \& McDougle, 2014; Leekam, Nieto, Libby, Wing, \& Gould, 2007). Interestingly, one previous ASD study, reported reduced global connectivity and local PAC despite similar event-related activity and oscillatory power between groups (Khan et al., 2013). Our data also suggest that local gamma power can be dysregulated without a discernible change in power. Future autism studies should therefore explore the precise regulation of gamma oscillations via cross-frequency coupling, rather than relying on measures of power alone.

\subsubsection{Neurocognitive Models of Perception in ASD}

More generally, the results of this study link with emerging theories of perception in autism. Predictive-coding accounts of cortical activity describe the passage of top-down predictions from higher to lower areas via feedback pathways, with prediction errors computed at each level of the hierarchy being passed forward via feedforward pathways (Friston, 2005). Predictive-coding accounts of autism suggest that differences in perception emerge from fewer or hyper-precise top-down predictions, such that perception is less influenced by prior knowledge and contextual cues (Palmer, Lawson, \& Hohwy, 2017; Pellicano \& Burr, 2012). Our data clearly support this proposal by showing reduced feedback connectivity in the visual cortex in autism. We also show that local gamma-band activity in primary visual cortex is decoupled from wider patterns of alpha-band phase coupling. This suggests that autistic perception can be characterised by differences in the hierarchical passage of information flow within the visual system (Palmer et al., 2017), captured through measures of oscillatory coupling. Where top-down information flow is reduced, this would force the perceptual system from predictive to reactive, with increased prediction error signalling and concomitant impacts on autistic symptoms (Kessler et al., 2016). This is supported by the observed correlations between feedback connectivity and PAC (Figure 3.6) and feedback connectivity and AQ score (Figure 3.7B).

\subsubsection{Clinical Implications, Limitations, and Future Work}

We note two limitations to this study. First, we did not collect a formal clinical assessment of autism, e.g. the ADOS. We therefore implemented strict participant exclusion criteria, only including autistic participants with a confirmed clinical diagnosis of ASD or Asperger's syndrome. Between groups, there were significant differences in autistic and sensory traits (Table 3.1). However, upon closer inspection of GSQ data (see Chapter 7, Figure 3.7.2), the ASD group showed a mixture of hyper- and hypo-sensitive traits between different sensory modalities making precise brain-behavioural correlations problematic. This may explain the lack of relationship between oscillatory connectivity and GSQ scores in autism (Figure 3.7A, C). For more discussion on this point see Chapter 7, Section 7.2. 
Brain-behaviour relationships might be better assessed using psychophysical tests of visual perception (Ashwin et al., 2009), combined with formal clinical assessments. Second, we constrained our connectivity analyses to two regions of interest (V1, V4) located early in the visual system, due to their hierarchical connectivity, and the low-level nature of the visual grating stimulus. However, we may have missed the opportunity to characterise more complex feedforward-feedback relationships in wider visual cortex. Future work should therefore include more ROls in combination with stimuli requiring participants to explicitly engage in feedback processing to constrain visual perception. This approach could be particularly useful with high-functioning individuals, and help characterise the neurophysiological basis of autistic perception (Kessler et al., 2016; Robertson \& Baron-Cohen, 2017).

The current results indicate that measures of oscillatory connectivity within the visual system, can elucidate atypical neural mechanisms in the autistic brain. Future research should elaborate on the current work by assessing these connectivity measures for their potential as stratification biomarkers of ASD in high-powered longitudinal studies (Loth et al., 2016). Due to the simplicity of the employed stimulus, the paradigm presented here could even be used in paediatric or non-verbal populations, since passive viewing of simple grating stimuli is sufficient for extracting the presented connectivity measures.

\section{Author Contributions}

RS, KK \& GR co-designed the study. RS collected the data, carried out the analysis, and was the primary author of this chapter. GGW helped with data acquisition and storage. JMS advised on details of MEG data analysis.

\section{Conflict of Interests Statement}

The authors wish to declare the research was conducted in the absence of any commercial or financial relationships that could be construed as a potential conflict of interest.

\section{Acknowledgments}

We wish to thank: Shu Yau for help with MRI data acquisition; and the Wellcome and Dr Hadwen Trusts for supporting MEG scanning costs. Robert Seymour was supported by a cotutelle PhD studentship from Aston University and Macquarie University. 


\title{
Chapter 4: Reduced Auditory Steady State Responses in Autism Spectrum Disorder
}

\section{Seymour, R.A ${ }^{1,2,3}$., Rippon, $\mathbf{G}^{1}$., Gooding-Williams, $\mathbf{G}^{1}$,. Sowman, P.F.2,3 \& Kessler, K'}

\author{
${ }^{1}$ Aston Brain Centre, School of Life and Health Sciences, Aston University, Birmingham, \\ B4 7ET.
}

${ }^{2}$ ARC Centre of Excellence in Cognition and Its Disorders, Macquarie University, Sydney, Australia, 2109.

${ }^{3}$ Department of Cognitive Science, Macquarie University, Sydney, Australia, 2109.

\subsection{Abstract}

Auditory steady state responses (ASSRs) are elicited by clicktrains or amplitudemodulated tones, which entrain auditory cortex at a specific modulation rate. Previous research has reported reductions in ASSRs at $40 \mathrm{~Hz}$ for autistic participants and firstdegree relatives of people diagnosed with ASD (e.g. Rojas et al., (2011); Wilson, Rojas, Reite, Teale, \& Rogers, (2007)). Using a 1.5s-long auditory clicktrain stimulus, designed to elicit an ASSR at $40 \mathrm{~Hz}$, this study attempted to replicate and extend these findings. Magnetencephalography (MEG) data were collected from 18 adolescent ASD participants and 18 typically developing controls. Results at the sensor and source-level showed that the ASD group had reduced oscillatory power at $40 \mathrm{~Hz}$ from $0-3.1 .5 \mathrm{~s}$ post-stimulus onset. Additionally, the ASD group had reduced inter-trial coherence (phase consistency over trials) at $40 \mathrm{~Hz}$ from $0.5-1.0 \mathrm{~s}$, for regions of interest in right/left auditory cortex. To investigate the developmental trajectories of ASSRs, data were separated into a group of older adolescents (age: 17-20) and younger adolescents (age: 14-17). For controls, there was an increase in $40 \mathrm{~Hz}$ power for older versus younger adolescents, but this was not the case for the ASD group. Finally we quantified the transient gamma-band response (tGBR) from $0-0.3 \mathrm{~s}$, in response to the clicktrain stimulus. However no group differences in the tGBR were found in sensor-space or source-space. Overall, the results are consistent with a specific reduction in ASSRs in ASD, rather than a generalised gamma deficit. We argue 
that this could reflect a developmentally relevant reduction in non-linear neural processing.

\subsection{Introduction}

Autism Spectrum Disorder (ASD) is a prevalent neurodevelopmental condition characterised by impairments in social interaction, disrupted communication and repetitive behaviours (APA, 2013). Although these features remain the primary diagnostic markers of ASD, the presence of sensory symptoms have recently been given a more central diagnostic role. This change reflects the observation that over $90 \%$ of ASD individuals experience hyper- and/or hypo-sensitive responses during sensory perception (Hazen, Stornelli, O’Rourke, Koesterer, \& McDougle, 2014; Leekam, Nieto, Libby, Wing, \& Gould, 2007). It has also been suggested that differences in low-level sensory processing contribute to the atypical developmental trajectories of higher-level cognitive functions in autism (Robertson \& Baron-Cohen, 2017). An understanding of the neural circuits involved will therefore prove fruitful for ASD research, and could even provide early diagnostic markers (Kessler, Seymour, \& Rippon, 2016; Roberts et al., 2010).

Dysregulated neural oscillations are a promising neural correlate of atypical sensory processing in autism. In particular, differences in high frequency gamma-band oscillations $(30-80 \mathrm{~Hz})$ have been reported in ASD across visual, auditory and somatosensory domains, see Chapter 1 and (Kessler et al., 2016; Simon \& Wallace, 2016). Gamma oscillations are generated through excitatory-inhibitory (E-I) neuronal coupling (Buzsáki \& Wang, 2012), which facilitates periods of pre and post-synaptic excitability alignment, thereby promoting efficient neural communication (Fries, 2015). Findings of atypical gamma oscillations in ASD may therefore reflect disrupted E-I interactions within cortical micro-circuits (Rubenstein \& Merzenich, 2003), and concomitant effects on local and global brain connectivity (Khan et al., 2013).

Within the context of auditory processing, findings of dysregulated gamma-band oscillations in ASD have been previously reported, reviewed in Chapter 1 Section 1.4.1 and Kessler et al., (2016). One prevalent approach to study auditory gamma-band activity non-invasively, is through amplitude modulated tones called "clicktrains" (Hari, Hämäläinen, \& Joutsiniemi, 1989). Such stimuli produce two distinct gamma-band responses. First, a transient gamma-band response (tGBR) is generated within the first 0.3 seconds after stimulus onset (Pantev, 1995). This tGBR is broadband $(30-60 \mathrm{~Hz})$, and is generated in primary and secondary auditory cortices. Second, clicktrain stimuli produce an auditory steady-state response (ASSR), in which neural populations in primary 
auditory regions are entrained to the modulation frequency for the duration of the clicktrain (Hari et al., 1989). In adults, the entrainment in primary auditory cortex is greatest for clicktrains modulated at $40 \mathrm{~Hz}$ (Pantev, Roberts, Elbert, Roß, \& Wienbruch, 1996). Measures of inter-trial coherence (ITC) can also be used to measure the ASSR, quantifying the degree of phase consistency across trials (Busch, Dubois, \& VanRullen, 2009). One advantage of ASSRs is their high test re-test reliability which approaches an intraclass correlation of 0.96 , even with a relatively small number of trials (McFadden et al., 2014; Tan, Gross, \& Uhlhaas, 2015). Furthermore, ASSRs are modulated by cognitive development, increasingly in power by approximately 0.01 ITC value per year, until early adulthood (Cho et al., 2015; Edgar et al., 2016). This makes the ASSR an ideal tool for studying auditory function in developmental conditions like ASD.

Two studies to date have measured ASSRs in ASD. Wilson, Rojas, Reite, Teale, \& Rogers, (2007) reported a reduction in left-hemisphere auditory ASSR power in a group of 10 autistic adolescents, using an early 37-channel MEG system. Another study reported reduced ITC in first-degree relatives of people diagnosed with autism (i.e. parents who had a child with an autism spectrum disorder), with maximal reductions at $40 \mathrm{~Hz}$ across both hemispheres (Rojas et al., 2011). Reductions to the ASSR could therefore be an ASD-relevant endophenotype. Additionally, the finding of reduced ITC suggests that dysregulated phase dynamics in bilateral primary auditory cortex could underlie reductions to the ASSR in ASD. However, measures of ITC have not been applied to study the ASSR in a group of autistic participants. Additionally it remains unclear whether reductions in ASSRs are bilateral (Rojas et al., 2011) or unilateral (Wilson et al., 2007) in nature.

This study attempted to replicate and extend previous findings showing a reduction in ASSR responses in autism (Rojas et al., 2011; Wilson et al., 2007). Data were collected from a group of 18 adolescent ASD participants and 18 typically developing controls using a 306-channel MEG system (Elekta Neuromag). An auditory clicktrain stimulus was presented binaurally to participants, made up of square wave clicks, delivered every $25 \mathrm{~ms}$, to elicit bilateral ASSRs at $40 \mathrm{~Hz}$. To investigate prolonged neural entrainment, clicktrain stimuli were presented for a total of 1.5 , rather than $0.5 \mathrm{~s}$ like previous studies (e.g. Rojas et al., 2011; Wilson, Rojas, Reite, Teale, \& Rogers, 2007). ASSRs were analysed over frequency and time, in order to investigate transient changes in $40 \mathrm{~Hz}$ power and inter-trial coherence. In addition, developmental changes in ASSRs were characterised by comparing a group of early adolescents (age: 13-17) to older adolescents (age: 17-20). As discussed above, clicktrain stimuli also elicit a broadband, transient gamma-band response (tGBR) within the first 0.3 s post-stimulus onset (Pantev, 
1995). However it remains unclear how tGBR is related to ASSRs in ASD (Rojas et al., 2011). Therefore in this study we compared both tGBR $(40-80 \mathrm{~Hz})$ and ASSRs at $(40 \mathrm{~Hz})$ across ASD and control groups.

\subsection{Materials and Methods}

\subsubsection{Participants}

Data were collected from 18 participants diagnosed with autism spectrum disorder and 18 age-matched typically developing controls (TDC), see Table 1.1 and Section 1.7.

\subsubsection{Experimental Procedures}

All experimental procedures complied with the Declaration of Helsinki and were approved by the Aston University, Department of Life \& Health Sciences ethics committee.

Participants and a parent/guardian gave written informed consent before participating in the study.

\subsubsection{Behavioural Assessments}

General non-verbal intelligence was assessed using the Raven's Matrices Task (Raven \& Court, 1998). The severity of autistic traits was assessed using the Autism Quotient (AQ) and sensory traits using the Glasgow Sensory Questionnaire (GSQ) (Robertson \& Simmons, 2013; responses further analysed in S2). Both AQ and GSQ scores were higher in the ASD group (Table 1). Participants also completed the Mind in the Eyes test (Baron-Cohen, Wheelwright, Hill, Raste, \& Plumb, 2001). There were no between group differences for this test. The Mind in the Eyes test has been recently criticised for measuring emotion recognition rather than an autism-specific deficit in mental state attribution (Oakley, Brewer, Bird, \& Catmur, 2016), and therefore these scores were not used to investigate correlations between brain patterns and questionnaire measures.

\subsubsection{Paradigm}

MEG was recorded whilst participants performed the same engaging sensory task as presented in Chapters $2 \& 3$, but here we analyse trials in which an auditory binaural clicktrain stimulus was presented. The auditory clicktrain was created from auditory square wave clicks, each of $2 \mathrm{~ms}$ duration delivered every $25 \mathrm{~ms}$ for a total of $1.5 \mathrm{~s}$. Clicktrains were presented at $80 \mathrm{~dB}$ binaurally through Etymotic MEG-compatible ear tubes. To keep participants engaged with the task, cartoon pictures of aliens or astronauts were presented after the auditory clicktrain, for a maximum of $0.5 \mathrm{~s}$. Prior to MEG acquisition, the nature of the task was fully explained to participants and several practice 
trials were performed. Accuracy of picture classification was above $95 \%$ for all participants.

\subsubsection{MEG Acquisition}

MEG data were acquired using a 306-channel Neuromag MEG scanner (Vectorview, Elekta, Finland) made up of 102 triplets of two orthogonal planar gradiometers and one magnetometer. All recordings were performed inside a magnetically shielded room at a sampling rate of $1000 \mathrm{~Hz}$. Five head position indicator (HPI) coils were applied for continuous head position tracking, and visualised post-acquisition using an in-house Matlab script. For MEG-MRI coregistration purposes three fiducial points, the locations of the HPI coils and 300-500 points from the head surface were acquired using a Polhemus Fastrak digitizer.

\subsubsection{Structural MRI}

A structural T1 brain scan was acquired for source reconstruction using a Siemens MAGNETOM Trio 3T scanner with a 32-channel head coil $(T E=2.18 \mathrm{~ms}$, TR=2300ms, $\mathrm{TI}=1100 \mathrm{~ms}$, flip angle $=9^{\circ}, 192$ or 208 slices depending on head size, voxel-size $=$ $0.8 \times 0.8 \times 0.8 \mathrm{~cm})$.

\subsubsection{MEG-MRI Coregistration and Cortical Mesh Construction}

MEG data were co-registered with participants' structural MRIs by matching the digitised head-shape data with surface data from the structural scan (Jenkinson \& Smith, 2001). Two control participants did not complete a T1 structural MRI and therefore a pseudo-MRI was used (see (Gohel, Lim, Kim, Kwon, \& Kim, 2017) for full procedure). The aligned MRIMEG images were used to create a forward model based on a single-shell description of the inner surface of the skull (Nolte, 2003), using the segmentation function in SPM8 (Litvak et al., 2011). The cortical mantle was then extracted to create a cortical mesh, using Freesurfer v5.3 (Fischl, 2012), and registered to a standard fs_LR mesh, based on the Conte69 brain (Van Essen 2012), using an interpolation algorithm from the Human Connectome Project (Van Essen et al., 2012; instructions here: https://goo.gl/3HYA3L). Finally, the mesh was downsampled to 4002 vertices per hemisphere.

\subsubsection{MEG Pre-Processing}

MEG data were pre-processed using Maxfilter (temporal signal space separation, .9 correlation), which supresses external sources of noise from outside the head (Taulu \& Simola, 2006). Further pre-processing steps were performed in Matlab 2014b using the Fieldtrip toolbox v20161024 (Oostenveld, Fries, Maris, \& Schoffelen, 2010). Firstly, for 
each participant the entire recording was band-pass filtered between $0.5-250 \mathrm{~Hz}$ (Butterworth filter, low-pass order 4, high-pass order 3) and band-stop filtered (49.5$50.5 \mathrm{~Hz} ; 99.5-100.5 \mathrm{~Hz}$ ) to remove residual $50 \mathrm{~Hz}$ power-line contamination and its harmonic. Data were epoched into segments of $4000 \mathrm{~ms}$ (1.5s pre, $1.5 \mathrm{~s}$ post stimulus onset, with $0.5 \mathrm{~s}$ of padding either side) and each trial was demeaned and detrended. Trials containing artefacts (SQUID jumps, eye-blinks, head movement, muscle) were removed if the trial-by-channel (magnetomer) variance exceeded $8 \times 10^{-23}$, resulting in the rejection, on average, of 3.4 trials per participant. Four MEG channels containing large amounts of non-physiological noise were removed from all analyses.

\subsubsection{Sensor-Level Spectral Power}

As the Neuromag MEG scanner contains two different sensor-types, with different scales and noise sensitivities, we opted to perform sensor-level analysis using only magnetomer data to improve the visualisation of results, and reduce issues with multiple comparisons (204 gradiometers vs 102 magnetometers). However for beamforming (see Section 4.3.10), both sensor-types were used to improve source localisation accuracy. M100 responses were calculated by averaging data across trials and comparing the evoked response $0.05-0.15 \mathrm{~s}$ post-clicktrain onset, to a $0.1 \mathrm{~s}$ baseline period. Sensor-level gamma power was analysed using the multi-taper method, as implemented in the Fieldtrip toolbox (Oostenveld et al., 2010). This has been shown to offer an optimal trade-off between time and frequency resolution, and is preferred to Morlet wavelets for high-frequency gammaband activity (Hoogenboom, Schoffelen, Oostenveld, Parkes, \& Fries, 2006; Muthukumaraswamy, 2013). Oscillatory power was calculated from $30-60 \mathrm{~Hz}$ using a $0.5 \mathrm{~s}$ sliding window (step size $0.02 \mathrm{~s}$ ) with $\pm 8 \mathrm{~Hz}$ frequency smoothing.

\subsubsection{Source-Level Spectral Power}

Source analysis was conducted using a linearly constrained minimum variance beamformer (Van Veen, van Drongelen, Yuchtman, \& Suzuki, 1997), which applies a spatial filter to the MEG data at each vertex of the cortical mesh. Both sensor types (magnetometers and gradiometers) were used for beamforming. Due to differences in noise between sensor-types, covariance matrix terms resulting from multiplying magnetomer and gradiometer data were removed (for more discussion on this, see this post on the Fieldtrip mailing list: https://bit.ly/2TVEILd). Beamformer weights were calculated by combining this covariance matrix with leadfield information, with data pooled across baseline and grating periods (see Figure 4.1). Following tSSS, sensor-level data had a rank of 64 or less, and therefore a regularisation parameter of lambda $5 \%$ was applied. 
Whilst the tGBR and ASSR originate from primary auditory cortex, both responses have different frequency ranges and underlying neural generators (Edgar et al., 2016).

Therefore we opted to use separate spatial filters, rather than single spatial filter based on the M100 as used in previous studies (Rojas et al., 2006, 2011; Wilson et al., 2007). This was based on recent work suggesting that beamformer weights should be optimised for specific data of interest (Barratt, Francis, Morris, \& Brookes, 2018) and effect topographies shown in Figure 4.2 indeed seem to indicate distinct generators. For more information see Figure 4.1.

\section{ASSR}

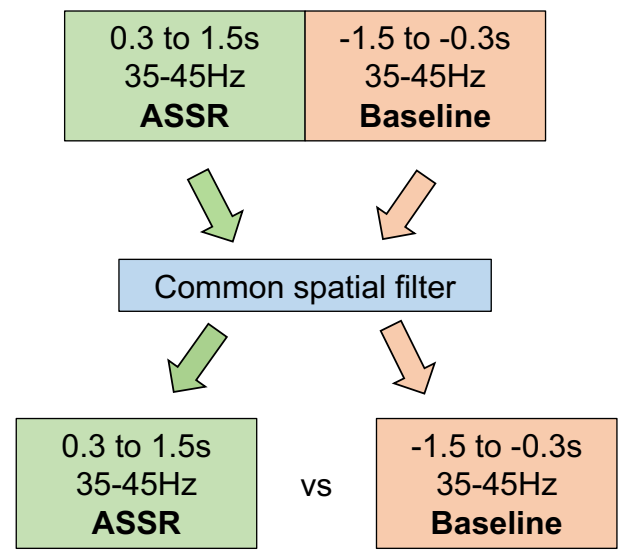

\section{tGBR}

\begin{tabular}{|c|c|}
\hline 0.05 to $0.3 \mathrm{~s}$ & -0.25 to $0 \mathrm{~s}$ \\
$30-60 \mathrm{~Hz}$ & $30-60 \mathrm{~Hz}$ \\
tGBR & Baseline \\
\hline
\end{tabular}

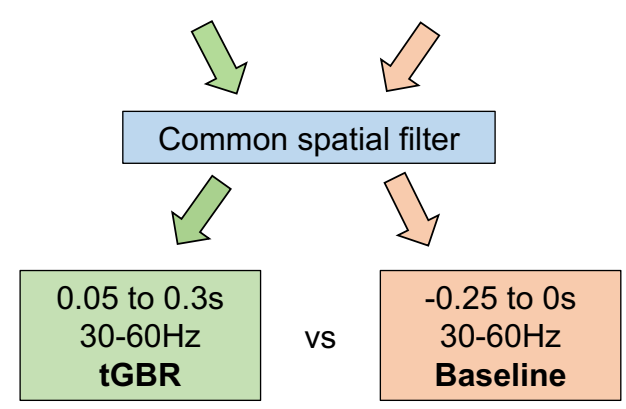

Figure 4.1 Procedure for Source Analysis. For ASSR beamforming, a common spatial filter was computed using data pooled across ASSR and baseline data. This common filter was then used to localise ASSR/baseline data separately. Crucially, this process was repeated for $t G B R$ data, but the common spatial filter was computed using a different time and frequency band of interest.

To localise the ASSR, data were band-pass filtered (Butterworth filter) between $35-45 \mathrm{~Hz}$. To capture induced rather than evoked visual activity, a period of 0.3-1.5s following stimulus onset was compared with a $1.2 \mathrm{~s}$ baseline period $(1.5-0.3 \mathrm{~s}$ before clicktrain onset). This avoids high-amplitude early-onset event-related fields such as the N100, occurring at $100 \mathrm{~ms}$ post-clicktrain onset, which could bias source localisation (see below). To localise the tGBR, data were band-pass filtered between $30-60 \mathrm{~Hz}$, and a period of $0.05-0.3$ s following clicktrain onset was compared with a 0.25 s baseline period (see Figure 4.1). 
Beamformers have been shown to be affected by correlated neural sources (e.g. bilateral auditory responses). Therefore, we opted to compute the covariance matrix on individual trials, rather than trial-averaged data (sensor-level data will be made more 'correlated' by averaging over trials). This procedure has been shown to produce sensible bilateral auditory localisations (see Fieldtrip 'Salzburg' tutorial: https://bit.ly/2GrB1mi). Results of the source analysis (see Figure 4.3) closely resembled the sensor-level data (see Figure $4.2 \mathrm{~B})$, and it is therefore unlikely that group differences were driven by differences in correlated sources.

\subsubsection{ROI definition}

To investigate time-frequency group differences, regions of interest (ROI) were selected in bilateral primary auditory (A1) cortices, defined using a multi-modal parcellation from the Human Connectome Project (Figure 4.32C, Glasser et al., 2016). To obtain a single spatial filter for each ROI (right A1 and left A1 separately), we performed a principal components analysis on the concatenated filters of each ROI, multiplied by the sensorlevel covariance matrix, and extracted the first component (see Schoffelen et al., 2017 for more details). Broadband $(0.5-250 \mathrm{~Hz})$ sensor-level data were multiplied by this spatial filter to obtain "virtual electrodes".

\subsubsection{A1 Spectral Power}

A1 gamma power (ASSR, tGBR) was analysed with the multi-taper method, using the same parameters as were used at the sensor-level. Statistical comparisons active>baseline, and control>ASD were performed using cluster-permutation tests (Maris \& Oostenveld, 2007).

\subsubsection{A1 Inter-trial Coherence}

Inter-trial coherence (ITC) is a measure of band-limited phase consistency across trials. An ITC value of 0 , indicates complete absence of phase consistency, whereas a value of 1 indicates perfect phase consistency across trials. At each time $t$ and frequency $f$, and for each trial $k$, ITC is calculated (Busch et al., 2009) as:

$$
I T C_{t, f}=\frac{1}{k} \sum_{n=1}^{k} e^{-i \varphi k(t, f)}
$$

Statistical comparison of active>baseline, and control>ASD was performed using clusterpermutation tests (Maris \& Oostenveld, 2007). 


\subsubsection{Statistical Analysis}

For MEG data, statistical analysis was performed using cluster-based permutation tests as implemented in the Fieldtrip toolbox, which have been shown to adequately control the type-I error rate for electrophysiological data (Maris \& Oostenveld, 2007). Cluster permutation tests consist of two parts: first an uncorrected independent t-test is performed, and all values exceeding a 5\% significance threshold are grouped into clusters. The maximum t-value within each cluster is carried forward. Second, a null distribution is obtained by randomising the condition label (e.g. ASD/TDC) 1000 times and calculating the largest cluster-level t-value for each permutation. The maximum $t$-value within each original cluster is then compared against this null distribution, with values exceeding a threshold of $\mathrm{p}<.05$ deemed significant.

\subsection{Results}

\subsubsection{Sensor-Level Results}

MEG data were analysed in three separate ways at the sensor-level (Figure 4.2A-C), based on the previous ASSR literature (Edgar et al., 2016; Rojas et al., 2006, 2008, 2011). Firstly the evoked M100 component was calculated by averaging trials from $0.05-$ $0.15 \mathrm{~s}$ (broadband frequency range). Second, the ASSR was analysed by calculating oscillatory power $(35-45 \mathrm{~Hz})$ between $0.3-1.5$ s post-stimulus onset. We avoided the first $0.3 \mathrm{~s}$ in order to separate the ASSR response from the low and high-frequency auditory evoked response. Thirdly the tGBR response was analysed by calculating oscillatory power between $0.05-0.3$ s post-stimulus onset, and from $30-60 \mathrm{~Hz}$. Each measure was baseline corrected using an equivalent time period. Results showed bilateral increases in amplitude (M100) and power (ASSR, tGBR), in a dipolar fashion, over frontal-middle sensors, as would be expected from an auditory stimulus. By statistically comparing control>ASD groups, it was found that the ASSR was larger in the control group, for left/right middle-frontal sensors, $\mathrm{p}<.034$ (Figure 4.2B, significant sensors highlighted). Both the evoked M100 and tGBR displayed no significant differences in amplitude (M100) or power (tGBR) between groups ( $p>.05$ ). 
A

\section{Auditory M100}
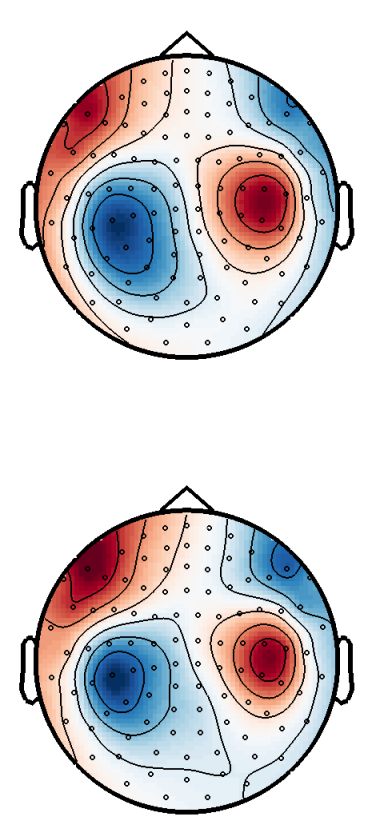
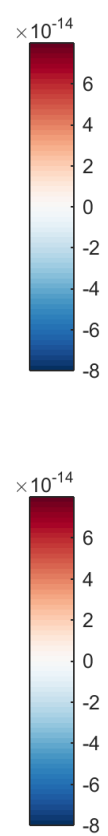

B 40Hz ASSR
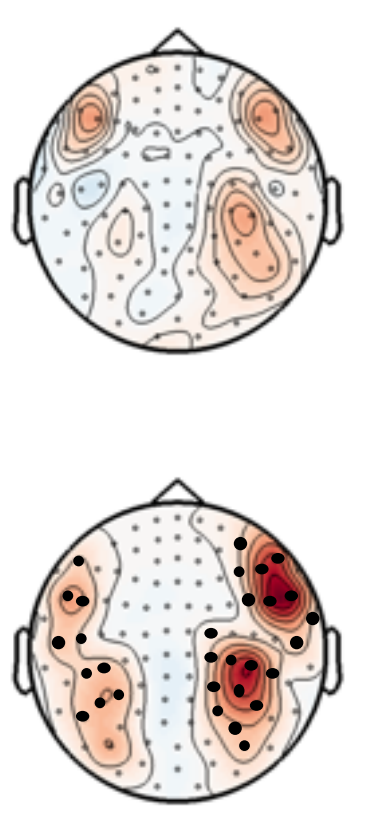
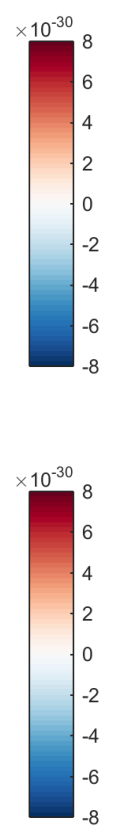

C

\section{0-60Hz tGBR}
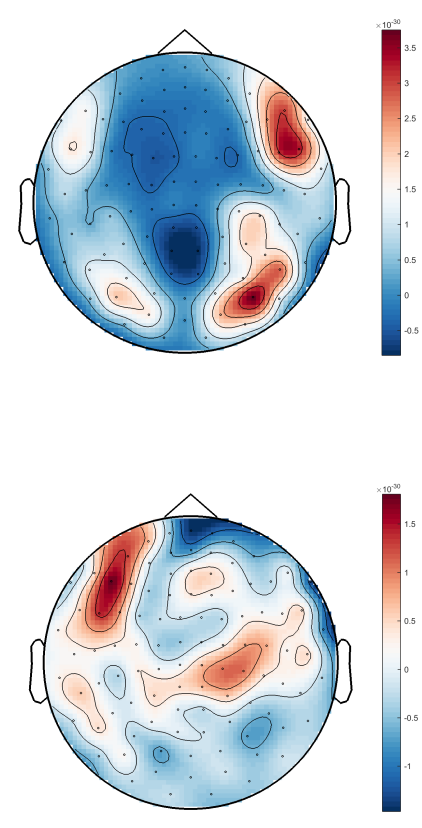

Figure 4.2. Sensor-level Analysis. (A) Group average topo-plot for the auditory M100 event-related field, magnetometers shown. (B) Group average topo-plot for auditory steady state responses (ASSR) at $40 \mathrm{~Hz}$. Sensors displaying a significant $(p<.05)$ group difference (control>ASD) in $40 \mathrm{~Hz}$ power are highlighted in bold. (C) Group average topo-plot of the transient gamma-band response (tGBR), 30-60Hz, 0.05-0.3s. Scales represent MEG field strength, baseline-corrected, with units of Tesla/ $\mathrm{cm}^{2}$. 
A

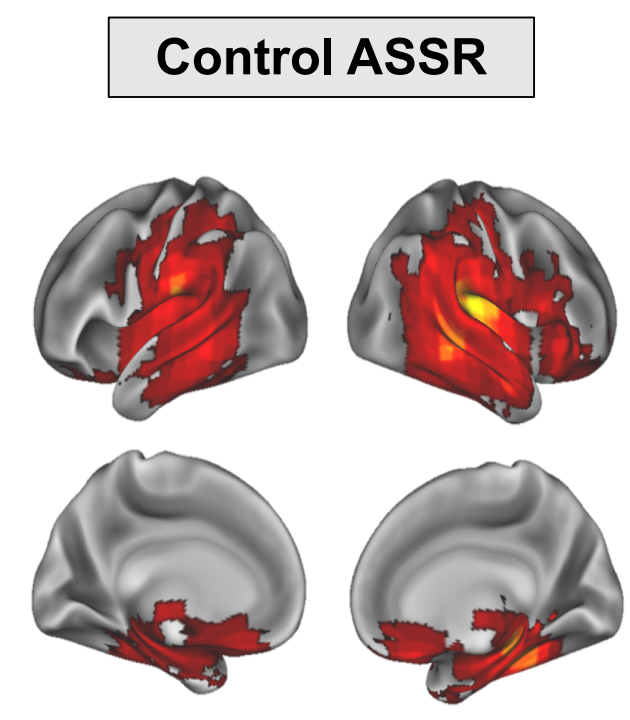

t-value
B

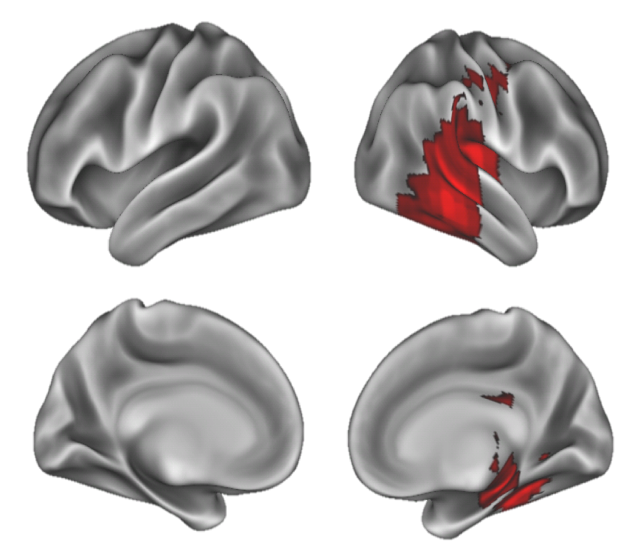

t-value

\section{ASD ASSR}

0.0

8.0

\section{Regions of Interest}

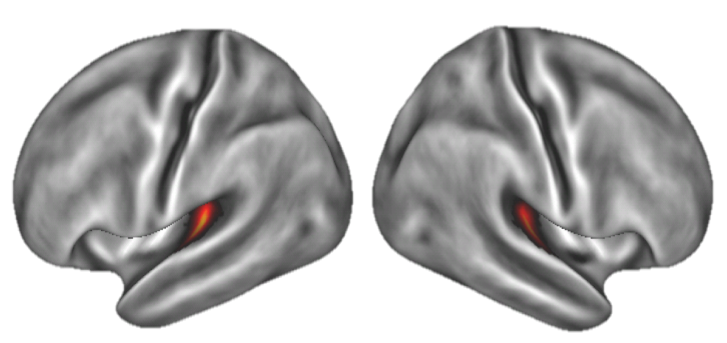

Figure 4.3. ASSR whole-brain source localisation. (A) The control group showed significant ( $p<.05)$ increases in $35-45 \mathrm{~Hz}$ power bilaterally in regions overlapping with auditory cortex. (B) The ASD group showed significant ( $p<.05)$ increases in $35-45 \mathrm{~Hz}$ power compared with baseline only in right hemisphere regions overlapping with auditory cortex. (C) Regions of interest for further analysis were defined in left and right primary auditory cortex (A1). 


\subsubsection{ASSR - Source-Level}

Cortical responses to the ASSR $(35-45 \mathrm{~Hz})$ were localised on a cortical mesh, using an LCMV beamformer, see Section 4.3 Methods \& Materials. We statistically compared 35$45 \mathrm{~Hz}$ power from $0.3-1.5 \mathrm{~s}$ post-clicktrain onset, to a $1.2 \mathrm{~s}$ baseline period, using clusterbased permutation testing to control for multiple comparisons (Maris \& Oostenveld, 2007). The control group showed significant, $p<.05$, increases in power for regions associated with auditory processing, including primary auditory cortex (Figure 4.3A). The same statistical comparison for the ASD group (clicktrain>baseline), also showed increases in power for right auditory regions, albeit with lower t-values than controls (Figure 4.3B). However, there were no significant increases in power for left-hemisphere auditory regions ( $p>.05)$ for the ASD group. For an alternative visualisation of results featuring whole-brain statistical maps uncorrected for multiple comparisons, see Appendix 2.

To investigate cortical ASSR time-frequency responses in greater detail, ROls were defined in bilateral auditory cortex (A1; Figure 4.3C). Oscillatory power was calculated in steps of $0.02 \mathrm{~s}$ using the multitaper method, and post-stimulus periods $(0-1.5 \mathrm{~s})$ were statistically compared to baseline periods (-1.5-0s). Control participants showed bilateral increases in power from $0.1-1.5 \mathrm{~s}, 35-45 \mathrm{~Hz}$ (Figure 4.4A), whereas the ASD group only showed increased power in right $\mathrm{A} 1$ (Figure 4.4B). Statistically comparing groups, it was found that the control group had higher $35-45 \mathrm{~Hz}$ power in both right $\mathrm{A} 1$ (Figure $4.4 \mathrm{C}$, $\mathrm{p}_{\text {cluster }}=.011$ ) and left $\mathrm{A} 1$ (Figure $4.4 \mathrm{D}, \mathrm{p}_{\text {cluster }}=.024$ ) than the ASD group. 
A

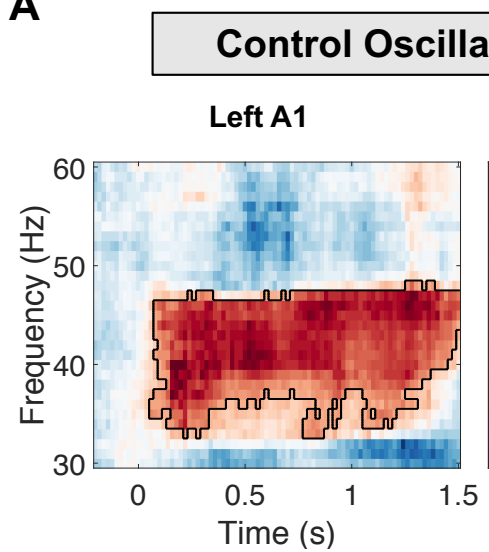

B

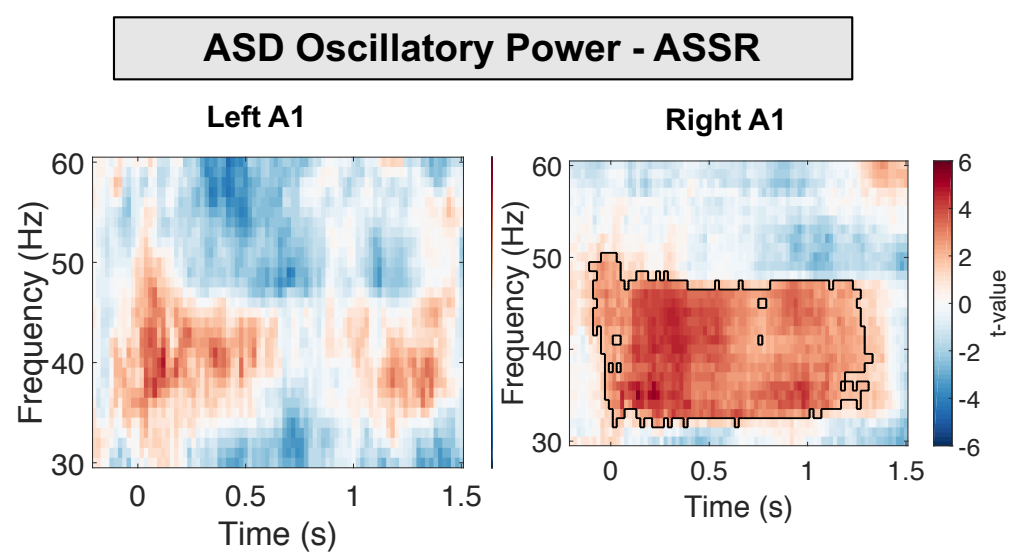

C

\section{Control > ASD Right A1}

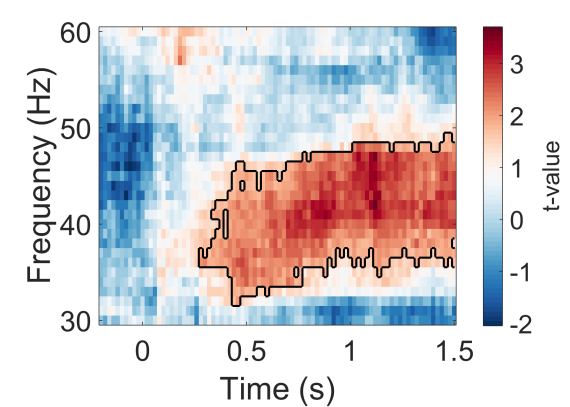

D

\section{Control > ASD Left A1}

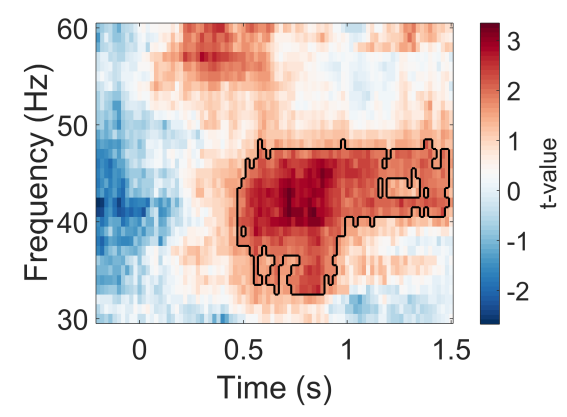

Figure 4.4. ASSR oscillatory power was analysed using a time-frequency approach in left and right primary auditory cortex, A1. (A) The control group showed significantly increased ASSR power $(p<.05)$ compared to pre-stimulus baseline between $35-45 H z$ in left and right $A 1$. (B) In contrast, the ASD group only showed significant increased ASSR power compared to baseline for right A1. (C-D) The control group had greater ASSR power from 0.41.5 s right $A 1, p=.011$, and $0.5-1.5$ s left $A 1, p=.024$, than the $A S D$ group. 
Next, we ran an exploratory post-hoc analysis to investigate hemispheric differences in the ASSR. For each ROI and participant, we calculated the percentage change in ASSR power from $35-45 \mathrm{~Hz}$, between $0.5-1.5 \mathrm{~s}$ post-clicktrain onset and a $1.0 \mathrm{~s}$ baseline period. These values were entered into a 2x2 ANOVA, with group (ASD, control) and hemisphere (left, right) as factors. Results showed a significant main effect of group, $F(1,68)=21.22$, $p<.001, \eta^{2}=.12$, and hemisphere $F(1,68)=15.63, p<.001, \eta^{2}=0.15$, but not a group*hemisphere interaction, $F(1,68)=1.780, p=.19, \eta^{2}=.017$. This suggests that the reduced ASSR power for the ASD group was not a function of hemisphere (Figure 4.5).

\section{Right and Left A1 $40 \mathrm{~Hz}$ power}

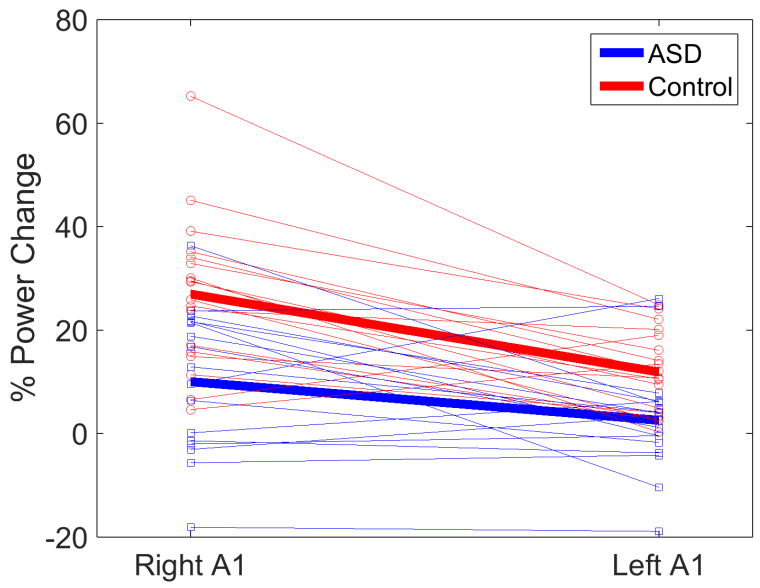

Figure 4.5. For left and right auditory cortex, the percentage change in ASSR power was calculated for $0.5-1.5$ s post-clicktrain onset versus a 1.0s baseline period. Data from each hemisphere and group is plotted separately (ASD: blue line; controls: red line). Thick lines represent the group mean whereas thinner lines represent individual data-points.

Next, inter-trial coherence (ITC) was calculated for the A1 ROls, using the same timefrequency approach as for power. We statistically compared post-clicktrain time-periods (0-1.5s) to baseline time-periods (-1.5-0s) which results in ITC values being converted to t-values. Both groups participants showed statistically significant, $p<.05$, increases in ITC from $0.1-1.5 \mathrm{~s}, 38-42 \mathrm{~Hz}$, across both left and right $\mathrm{A} 1$ (Figure 4.6A-B). Statistical comparison of ITC between groups showed that the control group had higher ITC in both right $\mathrm{A} 1$ (Figure 4.6C, $\mathrm{p}=.038$ ) and left $\mathrm{A} 1$ (Figure 4.6D, $\mathrm{p}=.020$ ), but only within a timewindow ranging from $0.5-1.0$ s post-stimulus onset. 
A

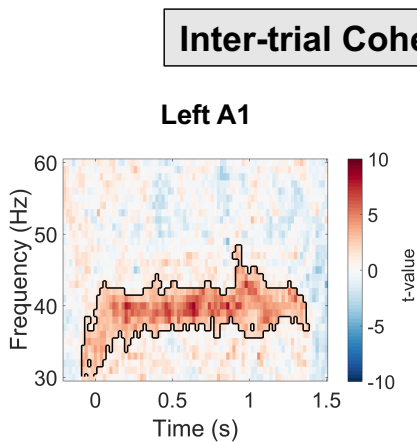

B

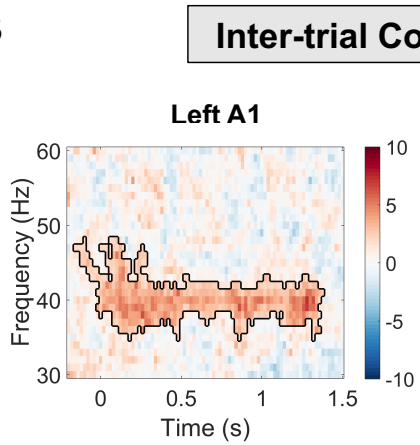

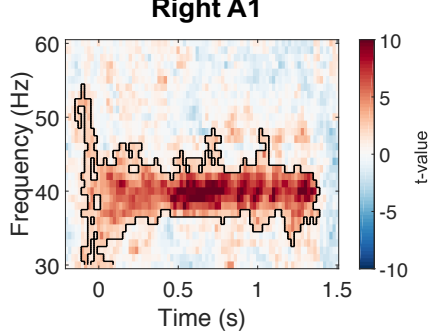

D
Control > ASD Right A1

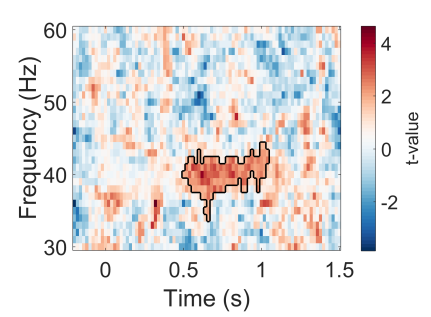

Control > ASD Left A1

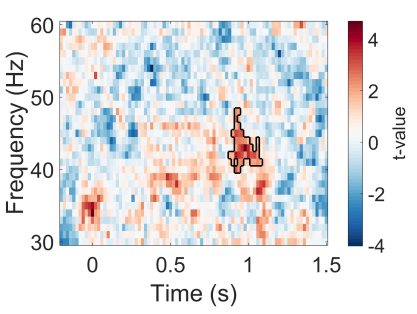

Figure 4.6. ASSR inter-trial coherence (ITC) was analysed across time (0-1.5s) and frequency $(30-60 \mathrm{~Hz})$ in left and right primary auditory cortex, A1. (A-B) Both groups showed increased ASSR ITC between $37-48 \mathrm{~Hz}$ in left and right A1. (C-D) Statistical comparison across groups revealed that the control group had significantly higher ASSR ITC from 0.4-1.0s right $A 1, p_{\text {cluster }}=.038$, and 0.9-1.11s left $A 1, p_{\text {cluster }}=.020$.

\subsubsection{ASSR - Behavioural Data}

To investigate brain-behaviour relationships we correlated ASSR power in right and left A1 with Autism Quotient (AQ) and Glasgow Sensory Questionnaire (GSQ) data. However, there were no significant correlations for either $A Q$ (Figure 4.7A, $r=.14, p=.586$ ) or $G S Q$ (Figure 4.7B, $r=-.22, p=.381$ ).

To investigate the developmental trajectory of ASSRs in our data (Rojas et al., 2006), we performed a median split by age (14-17 vs. 17-20, 9 participants in each mini-group). These data were entered into an ANOVA with group (ASD, control) and age (young, old) as factors. Results showed a main effect of group, $F(1,32)=16.44, p<.001, \eta^{2}=0.30$. However there was only a trend for an effect of age, $F(1,32)=2.856, p=.101, \eta^{2}=0.053$, and group*age interaction, $F(1,32)=2.760, p=.106, \eta^{2}=0.051$. Performing a simple main effects by age, it was found that for the control group, older adolescents had significantly higher ASSR power compared with younger adolescents, $F(1,32)=5.615, p=.024$. However this was not the case for the ASD group $F(1,32)=4.09 \times 10^{-4}, p=.984$. 
A

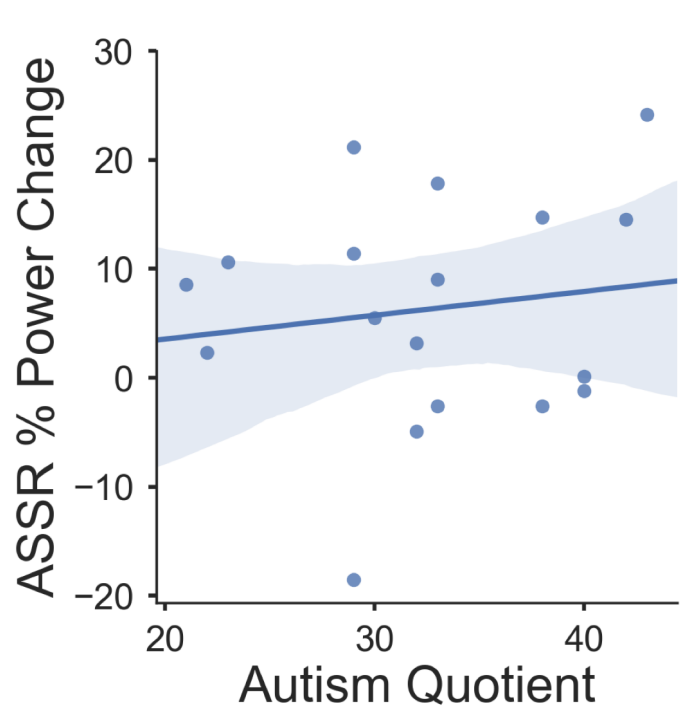

B

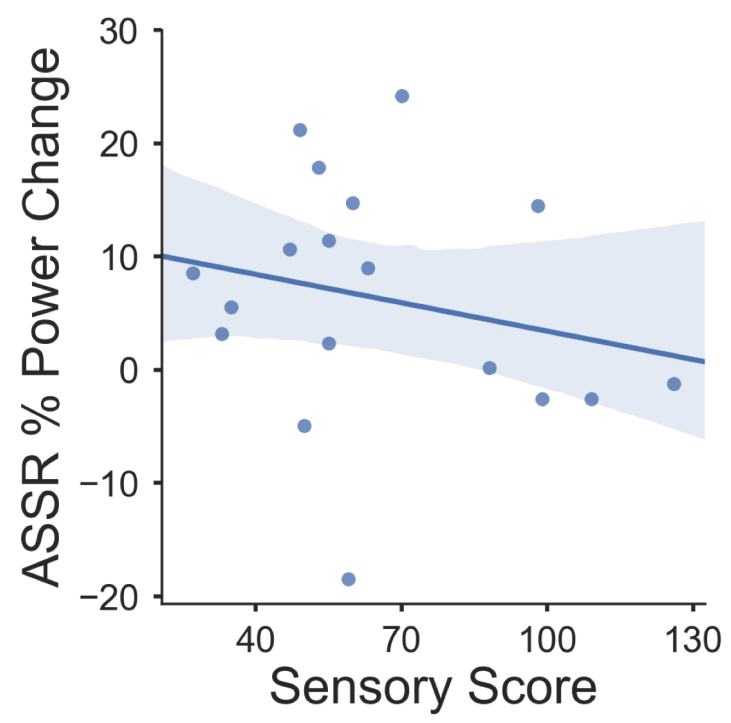

C

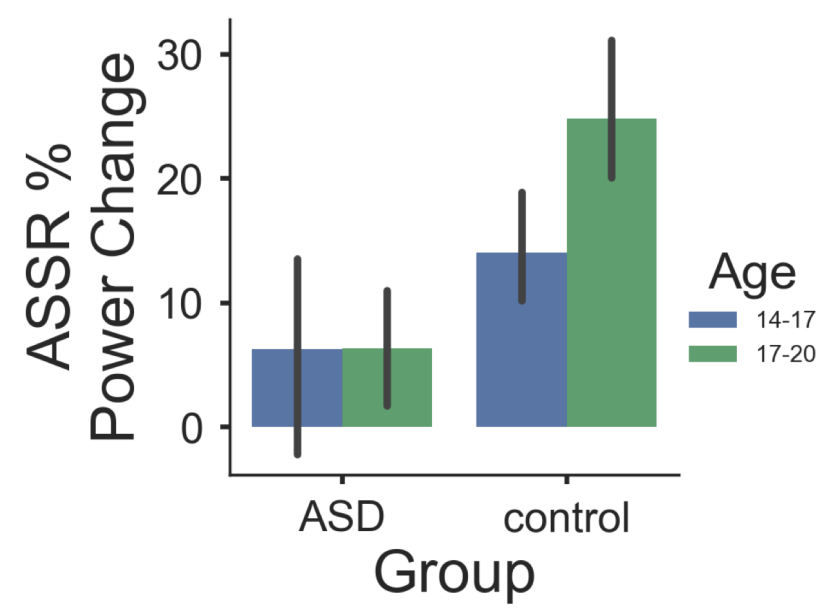

Figure 4.7. (A-B) Scatter plots for ASSR power, averaged across left and right A1, and Autism Quotient / Sensory Scores. The shaded region indicates $95 \%$ confidence intervals. (C) Bar plot for ASSR power in young adolescents (age: 13-17) compared with older adolescents ( $N=9$, age: 1720). There were 9 participants in each sub-group (ASD/control and young/old adolescents). Error lines indicate $95 \%$ confidence intervals. 


\subsection{4 tGBR - Source-Level}

Transient gamma-band responses to the auditory clicktrain were localised using a beamforming approach, see Section 4.3. We statistically compared whole-brain power maps $(30-60 \mathrm{~Hz})$ from $0.05-0.3$ s post-clicktrain onset to a 0.25 baseline period (Maris \& Oostenveld, 2007). Results showed significant, $p<.05$, increases in power for both groups, in brain regions that overlapped the bilateral auditory regions (Figure 4.8A-B).

Paralleling the ASSR analysis, ROls were defined in bilateral A1. For each ROI and participant, we calculated the percentage change in tGBR power from $30-60 \mathrm{~Hz}$, between $0.0-0.3$ s post-clicktrain onset and a $0.3 \mathrm{~s}$ baseline period. These values were entered into a 2x2 ANOVA, with group and hemisphere as factors (Figure 4.8C). Results showed no significant main effect of: group, $F(1,68)=0.681, p=.41, \eta^{2}=.010$; hemisphere, $F(1,68)=$ $0.252, p=.62, \eta^{2}=.004$; and no significant group*hemisphere interaction, $F(1,68)=0.651$, $p=.42, \eta^{2}=.009$, i.e. tGBRs are not significantly different across groups or hemispheres. 
A

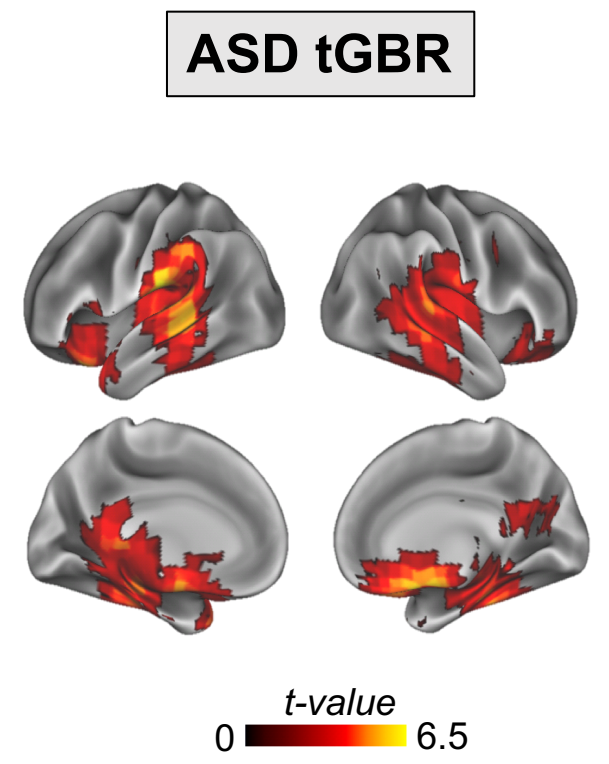

B

\section{Control tGBR}

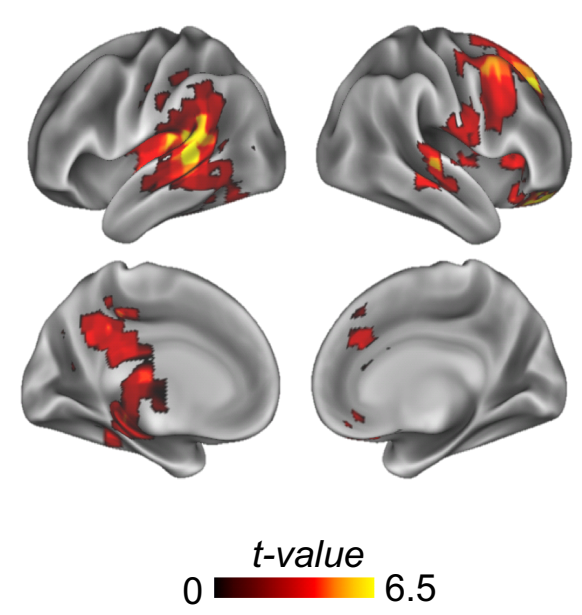

\section{A1 tGBR}

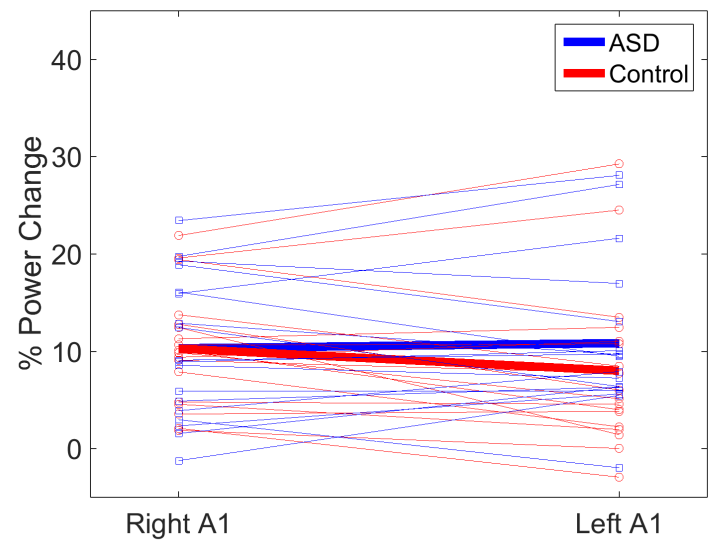

Figure 4.8. Source-space transient gamma-band responses (0-0.3s post-stimulus onset; $30-60 \mathrm{~Hz})$. (A-B) Both groups show statistically significant $(p<.05)$ increases in $t G B R$ power versus baseline in regions overlapping the auditory cortices $(C)$ tGBR was plotted separately across hemispheres and group (ASD: blue line; controls: red line). Thick lines represent the group mean whereas thinner lines represent individual data-points. 


\subsection{Discussion}

This study examined the oscillatory basis of auditory steady state responses (ASSRs) and transient gamma-band responses (tGBR) in a group of autistic adolescents. We utilised robust source-localisation methods and analysed auditory responses across both frequency and time. Compared to the ASSR in the control group, we found reduced $\sim 40 \mathrm{~Hz}$ power at both the sensor and source level for the ASD group, for both left and right primary auditory cortices. A developmental increase in ASSR power during adolescence was found in controls, but not the autistic group. Furthermore, there was reduced inter-trial coherence for the autistic group at $40 \mathrm{~Hz}$, suggesting that phase dynamics in A1 were less consistent over time. Our results are consistent with the view that auditory brain responses in autism are locally dysregulated (Kessler et al., 2016), especially during sustained gamma-band entrainment $(<0.4 \mathrm{~s})$.

\subsubsection{Auditory Steady State Responses (ASSR) in Autism}

Our results partially replicate two previous studies showing reduced ASSRs in autistic adolescents (Wilson et al., 2007) and first-degree relatives of people diagnosed with autism (Rojas et al., 2011). Whilst our study shows reductions in $40 \mathrm{~Hz}$ power across both hemispheres (Figure 4.5), Wilson et al., (2007) observed a selective left-hemisphere reduction in power. This might be due to monaural stimulation, used in Wilson et al., (2007), producing larger hemispheric asymmetries as compared to binaural auditory stimulation (Ross, Herdman, \& Pantev, 2005). Future work is clearly needed to clarify hemispheric asymmetries in ASSR power for ASD populations (Ross et al., 2005).

Our results build on the previous literature in several ways. Firstly, by examining sustained ASSRs from $0-1.5 \mathrm{~s}$ we found that group differences emerged after $0.4 \mathrm{~s}$ post-stimulus onset (Figure 4.4), suggesting that, when driven at gamma frequencies, $\mathrm{A} 1$ becomes increasingly dysregulated in ASD compared to controls in a time dependent manner. This raises the intriguing possibility that sustained, rather than transient, oscillatory activity at gamma-frequencies is affected in autism, perhaps reflecting synaptic dysfunction and an imbalance between excitatory and inhibitory populations of neurons (Rubenstein \& Merzenich, 2003). To investigate this further, future work could parametrically modulate clicktrain duration and intensity. Secondly, our results were complimented by measures of ITC, which showed reduced phase consistency in the autistic group from $0.5-1.0$ s poststimulus onset (Figure 4.6). Reduced phase consistency may reflect more variable or idiosyncratic neural responses in autism (Hahamy, Behrmann, \& Malach, 2015), as previously reported for evoked data (Dinstein et al., 2011). Importantly, measures of ITC are normalised by amplitude and more robust to noisy data (Tan et al., 2015). Therefore, 
the correspondence between power and ITC, albeit in a smaller time-window for ITC, strengthens the claim of reduced ASSRs in autism. Thirdly, by examining age-related changes in ASSRs, we found that $40 \mathrm{~Hz}$ power increased from early to late adolescence for control participants (Figure 4.7C). This developmental increase in power is well characterised (Cho et al., 2015; Edgar et al., 2016; Rojas et al., 2006), and may reflect the continuing development of superficial layers of cortex where gamma-band oscillations originate (Moore \& Linthicum Jr, 2007). However, this developmental effect was absent in the ASD group (simple main effects analysis, see Section 4.4.3). This could potentially indicate atypical trajectories of gamma-band maturation in autism, in line with developmental disconnection theories of autism (Geschwind \& Levitt, 2007). Widespread brain maturation continues into adolescence (Blakemore, 2012), and therefore divergent ASSR trajectories in this age range could act as important autism-relevant markers of intervention efficacy (Mamashli et al., 2018). However given the very small sample sizes after further segregation by age ( $\mathrm{N}=9$ in each sub-group), it is not surprising that the age ${ }^{*}$ group interaction did not reach significance $(p=.106)$, thus, calling for a cautious interpretation of the data and strongly emphasising future research with larger sample sizes.

\subsubsection{Transient Gamma-Band Responses in Autism}

Unlike ASSRs, transient gamma-band $(30-60 \mathrm{~Hz})$ responses to the clicktrain stimuli were not different between groups (Figure 4.8). Whilst one previous study using sinusoidal tones reported decreased tGBRs for the first-degree relatives of autistic people, a later study using auditory clicktrains, found no group differences in either power or ITC (Rojas et al., 2011). More generally, findings of transient/evoked gamma-band power across sensory domains are very mixed, with both increases and decreases reported (reviewed in Kessler et al., 2016). The divergence between steady-state and transient gamma in this study has implications for potential oscillopathies in ASD, as differences in gamma power may depend on the time-period under investigation as well as the underlying neural circuits generating gamma oscillations (Edgar et al., 2016).

\subsubsection{ASSRs as Markers of Dysregulated Local Activity}

There has been recent interest in characterising atypical patterns of gamma-band oscillations in autism, due to their link with local cortical function and connectivity (Kessler et al., 2016). The precise E-I mechanisms underlying gamma generation are well characterised, for a review see Buzsáki \& Wang (2012). Of particular importance is the functional inhibition of pyramidal neurons by fast-spiking interneurons (Buzsáki \& Wang, 2012) via binding of the neurotransmitter gamma-aminobutyric acid (GABA). Interestingly, 
there is emerging evidence showing GABA dysfunction in autism (Coghlan et al., 2012). Reduced gamma-band steady-state responses in autism may therefore reflect dysregulated neuronal inhibition, resulting in E-I imbalance (Rubenstein \& Merzenich, 2003). To quantify the precise mechanisms underlying reduced gamma-band ASSRs, future studies could utilise dynamic causal modelling of A1 neuronal circuits (Moran et al., 2009), combined with parametric modulations of ASSRs (e.g. duration, frequency) and participant attention (Ross, Picton, Herdman, \& Pantev, 2004).

It should also be noted that ASSRs are not simply generated via the linear accumulation of transient evoked responses (Azzena et al., 1995; Pantev et al., 1996; Santarelli et al., 1995). Instead, the ASSR may reflect a sustained non-linear neural response at the input stimulation frequency and its harmonics, peaking at the system's preferred modulation rate (Pantev et al., 1996). In support of this, Edgar et al., (2016) report that in children, ASSRs are difficult to detect, despite measurable auditory evoked responses. Similarly, our data show intact auditory evoked fields and transient gamma-band responses in autism, despite a reduced ASSR. Rather than a generalised gamma-band dysfunction in autism, our data suggest a more nuanced reduction in the non-linear dynamics underlying steady-state auditory gamma (Rojas et al., 2011). Interestingly, an MEG study examining somatosensory processing in autism showed reduced frequency harmonics at $50 \mathrm{~Hz}$, suggesting that non-linear aspects of local cortical processing could be dysregulated across sensory domains in ASD (Khan et al., 2015).

\subsubsection{Limitations}

We did not collect a formal clinical assessment of autism from our participants, e.g. the ADOS. We therefore implemented strict participant exclusion criteria, only including autistic participants with a confirmed clinical diagnosis of ASD or Asperger's syndrome. Between groups, there were significant differences in autistic and sensory traits, measured using two self-report questionnaires (Table 1). However, upon closer inspection of behavioural data, the ASD group showed a mixture of hyper- and hypo-sensitive traits between different sensory modalities making precise brain-behavioural correlations problematic. This may explain the lack of relationship between oscillatory connectivity and $A Q / G S Q$ scores in autism (Figure 4.7A-B). Brain-behaviour relationships might be better quantified using psychophysical tests of auditory perception and formal clinical assessments. 


\section{Author Contributions}

RS, KK \& GR co-designed the study. PFS helped with data interpretation and gave feedback on a draft of this chapter. GGW helped with data collection and analysis and provided feedback on a draft. RS collected the data, carried out the analysis, and was the primary author of this chapter.

\section{Conflict of Interests Statement}

The authors wish to declare the research was conducted in the absence of any commercial or financial relationships that could be construed as a potential conflict of interest.

\section{Acknowledgments}

We wish to thank: the volunteers who gave their time to participate in this study; $\mathrm{Dr}$ Shu Yau for help with MRI data acquisition; Dr Jon Brock for intellectual contributions to experimental design; and the Wellcome Trust, Dr Hadwen Trust and Tommy's Fund for supporting research costs. Robert Seymour was supported by a cotutelle PhD studentship from Aston University and Macquarie University. 


\title{
Chapter 5: Testing Predictive Coding Theories of Autism Spectrum Disorder Using the Auditory Oddball Paradigm - An MEG Study
}

\author{
Seymour, R.A ${ }^{1,2,3}$., Kessler, $K^{1}$. He, $W^{2,3}$., \& Sowman, \\ P.F ${ }^{2,3}$.
}

${ }^{1}$ Aston Brain Centre, School of Life and Health Sciences, Aston University, Birmingham, B4 7ET.

${ }^{2}$ ARC Centre of Excellence in Cognition and Its Disorders, Macquarie University, Sydney, Australia, 2109.

${ }^{3}$ Department of Cognitive Science, Macquarie University, Sydney, Australia, 2109.

\subsection{Abstract}

There is increasing interest in understanding the perceptual and sensory symptoms of Autism Spectrum Disorder (ASD) from a predictive-coding perspective. One theory argues that sensory differences result from reduced top-down predictions ('hypo-prior' accounts); whilst another theory suggests that ASD is characterised by the atypical the weighting of prediction errors ('hyper-precision' accounts). To test the neural basis of these theories, we used magnetoencephalography (MEG) in combination with an auditory oddball paradigm. Evoked 'mismatch' field (MMF) responses were calculated by comparing brain activity to 'standard' tone-sequences with unexpected 'deviant' tone-sequences. We investigated MMF responses to the omission of an expected tone (omiMMF); and the unexpected presentation of a deviant tone half the duration of standard tone (durMMF). Data were collected from 16 adolescent participants diagnosed with ASD, and 16 agematched controls. Results showed that compared with age-matched controls, ASD participants had reduced omiMMF responses, within bilateral superior-temporal gyrus. However, no group differences in durMMF responses were found. The oscillatory basis of omission responses were investigated using inter-trial coherence (ITC). However, no 
group differences in ITC were found. To test precision-weighting theories of ASD, the auditory tones were presented in babble-noise, however this resulted in extinction of MMF effects across both groups. Overall, the finding of reduced omiMMF responses provides evidence for reduced top-down predictions in ASD, supporting hypo-prior accounts of ASD. However more research is required to understand the oscillation-based functional connectivity underlying these findings.

\subsection{Introduction}

Autism Spectrum Disorder (ASD) is a neurodevelopmental condition, characterised by impairments in social interaction and communication, as well as the presence of repetitive behaviours and stereotyped interests (APA, 2013). Although these features remain the primary diagnostic markers of $A S D$, co-occurring sensory symptoms have recently been weighted more heavily. Over $90 \%$ of ASD individuals experience hyper- and/or hyposensitive responses to certain stimuli (Leekam, Nieto, Libby, Wing, \& Gould, 2007), which can significantly interfere with activities of daily living (Jones, Quigney, \& Huws, 2003). Additionally, behavioural research has shown low-level differences in sensory and perceptual processes in ASD, reviewed in (Robertson \& Baron-Cohen, 2017). By beginning to understand the neural circuitry that gives rise to these sensory difficulties, a clearer picture of the neurobiological basis of ASD might emerge. Furthermore, sensory anomalies and their neural underpinnings could even provide early diagnostic markers of ASD (Kessler, Seymour, \& Rippon, 2016; Roberts et al., 2010).

Two influential theoretic accounts which posit a mechanistic basis for the sensory symptoms in ASD are: 1) The "weak central coherence account", which suggests that autism is characterised by a perceptual style biased against global and holistic processing (Happé, 2005), and; 2) the "enhanced perceptual functioning" account, which suggests that autistic perception is biased towards local detail, due to enhanced "perceptual operations" (Mottron, Dawson, Soulieres, Hubert, \& Burack, 2006). However, the focus of these theories is an imbalance in the distinction between local and global perception, and hence neither attempts to explain the breadth of sensory symptoms, reported by autistic people, including both hypo- and hyper-sensitivities (Leekam et al., 2007; Robertson \& Baron-Cohen, 2017). More recently, it has been proposed that ASD sensory differences can be explained through Bayesian or predictive coding models of perception (Pellicano \& Burr, 2012; Rao \& Ballard, 1999). Such models describe the cognitive processes undertaken by the brain in terms of Bayesian inference; essentially the brain can be thought of as a Bayesian machine which seeks to minimise the difference between prior expectations or "predictions" and incoming sensory information. This process is achieved 
through the construction of generative, hierarchical models, in which feedback pathways carry predictions, and feedforward pathways carry the residual error (the difference) between the neural representation of predictions and that of the sensory consequences of events. This difference is termed the "prediction error" (Friston, 2008). Prediction errors, weighted according to their expected precision, can then be used to update future predictions, and refine internal representations of how events and actions map onto sensations. In this way, complex perceptual processes can be implemented through the learning of statistical regularities in the world (Friston, 2005). Applying a predictivecoding/Bayesian inference framework to ASD, (Pellicano \& Burr, 2012), and later (Van Boxtel \& Lu, 2013), suggested that differences in perception in ASD may be the result of weaker top-down prediction, a hypothetical mechanism termed "hypo-prior". Alternatively, it has been suggested that ASD is characterised by the atypical context-sensitive weighting of prediction-errors, i.e. precision (Lawson, Rees, \& Friston, 2014; Van de Cruys et al., 2014). Where an environment is "noisy", i.e. the signal to noise ratio is lower, the theory states that autistic people will struggle to use top-down predictions to adequately constrain bottom-up sensory information processing. This would result in excessive prediction error signals, and potentially increased feedforward connectivity in ASD (Kessler et al., 2016; Khan et al., 2015). There is emerging behavioural evidence to support this "hyper-precision" view of ASD (Goris et al., 2018; Lawson, Aylward, Roiser, \& Rees, 2017; Lawson, Mathys, \& Rees, 2017), with relevance for understanding both the sensory and social symptoms of ASD, see (Palmer, Seth, \& Hohwy, 2015)), as well as for the design of interventions (Friston, 2017). However, direct neural evidence to support a predictive-coding basis for ASD is sparse.

One of the dominant paradigms in predictive coding research is the auditory oddball paradigm, in which repetitive 'standard' sounds are interspersed with infrequent and unexpected 'deviant' sounds. Compared with standards, deviant sounds produce a larger event-related potential/field, termed the mismatch negativity (MMN, as measured with EEG) or mismatch field (MMF, as measured with MEG). The auditory MMN/F can be elicited by a number of acoustic properties, including unexpected changes in intensity, duration and frequency (Näätänen, 1995; Näätänen, Paavilainen, Rinne, \& Alho, 2007). The underlying neural generators of the MMN/F have been well characterised, and consist of an interconnected network of bilateral primary and secondary auditory cortices and extensions into inferior frontal regions (Garrido et al., 2008; Garrido, Kilner, Stephan, \& Friston, 2009). As well as oddball responses to unexpected deviant sounds, the brain also responds to the unexpected omission of sounds (Bendixen, SanMiguel, \& Schröger, 2012; Raij, McEvoy, Mäkelä, \& Hari, 1997). The omission MMN/MMF is unlikely to be driven by 
neural adaptation, and therefore represents a more direct measure of prediction violations (Sauer et al., 2017). Compared with deviant responses, the omission MMN/MMF has similar neural generators, but a slightly different connectivity pattern (Chennu et al., 2016). In this study we examined both "omission" and "deviant" oddball responses to investigate predictive processes in ASD.

Research utilising auditory oddball paradigms in ASD populations has produced inconsistent results. These inconsistencies could be due to the fact that deviant auditory MMN/F responses are driven by both violations of prediction and the rebound from neural adaptation (Garrido et al., 2009). Omission MMN/F responses, which have been argued to solely reflect prediction error (SanMiguel, Widmann, Bendixen, Trujillo-Barreto, \& Schröger, 2013), may therefore prove less complicated and hence more robust marker of predictive coding; yet, to our knowledge, omission MMN/F responses have not been studied in ASD. Most auditory oddball studies in ASD populations to date have utilised EEG, with the data subsequently collapsed to one electrode (usually Fz). Whole-head magnetoencephalography (MEG), in contrast, has much higher signal-to-noise compared with EEG, especially when combined with source reconstruction algorithms for specific regions of interest (Hillebrand \& Barnes, 2005). Several MEG studies have found reduced (Mamashli et al., 2017; Tecchio et al., 2003) and delayed (Cardy, Flagg, Roberts, \& Roberts, 2005; T. P. L. Roberts et al., 2011) auditory oddball responses in ASD. Furthermore, one study has reported that attention interacts with auditory oddball responses in ASD, with reduced MMN/Fs only when attention was directed away from the auditory tones (Dunn, Gomes, \& Gravel, 2008).

In this study, we investigated predictive coding in ASD using an auditory oddball paradigm (Recasens \& Uhlhaas, 2017), whilst measuring neural activity with MEG. Data from 16 adolescent participants diagnosed with ASD and 16 age-matched controls was analysed, with data from a further 4 ASD participants collected but excluded from analysis. There were two types of 'oddball' trial: a duration mismatch in which the final tone was half the length of the standard one; and an omission mismatch in which the final tone was not presented at all. This paradigm produces MMF responses which are highly reliable (interclass correlations of $0.81-0.90$ (Recasens \& Uhlhaas, 2017)), and detectable at the single-subject level. In this study, the tones were presented both against a silent background and when embedded within background noise. Using a different oddball paradigm, (Mamashli et al., 2017) reported that ASD participants show a reduced MMF and reduced fronto-temporal connectivity when tones are presented in babble-noise. The same babble-noise was used for this study, see (Dreschler, Verschuure, Ludvigsen, \& 
Westermann, 2001). This allowed us to test the precision-weighting dysfunction theory of ASD (Lawson et al., 2014; Palmer, Lawson, \& Hohwy, 2017), as tones presented in noise result in neural responses with lower signal to noise and therefore should generate less precise top-down predictions. We hypothesised that the ASD group would show reduced MMF responses to the duration and omission oddball stimuli, especially when tones were presented against background noise, in accordance with predictive coding theories of ASD (Lawson et al., 2014; Mamashli et al., 2017; Palmer et al., 2017).

Due to the focus on oscillations in this thesis (see Chapter 1), we additionally analysed the data in the frequency domain. Event-related fields are related to phase reset (i.e. consistent phase across trials), which can be quantified using inter-trial coherence (ITC, also see Chapter 4). In other words, we were interested in quantifying the extent to which the presentation of a deviant vs standard tone, resets ongoing oscillatory activity within auditory regions. Interestingly, ITC has been previously been used to investigate the differences in the MMF response between schizophrenia patients and controls (Lee et al., 2016).

Lastly, due to the focus on directed connectivity in this thesis (see Chapter 3), a directed functional connectivity analysis was conducted using Granger Causality (GC). It was hypothesised that the ASD group would show reduced top-down connectivity from frontal to primary auditory regions (Mamashli et al., 2017), reflecting connectivity patterns that suggest reduced prediction (Friston, 2011; Kessler et al., 2016).

\subsection{Materials and Methods}

\subsubsection{Participants}

Data were collected from 20 participants diagnosed with ASD and 20 age-matched typically developing controls, see Table 1 . ASD participants had a confirmed clinical diagnosis of ASD or Asperger's syndrome from a paediatric psychiatrist. In addition, participants completed an online edition of the Autism Quotient questionnaire (BaronCohen et al., 2001), which assesses the level of autistic traits. 18/20 ASD participants had a score over 25; which has been defined as the arbitrary threshold corresponding to clinically-relevant levels of autistic traits. Participants were excluded if they were taking psychiatric medication or reported epileptic symptoms. Control participants were excluded if a sibling or parent was diagnosed with ASD. Data from 4 ASD participants was collected but excluded, due to an intolerance to MEG resulting in movement over $5 \mathrm{~mm}$ (3) and a 
technical issue with marker-coil measurement (1). The corresponding age-matched control data were also excluded from the analysis.

All experimental procedures complied with the Declaration of Helsinki and were approved by the Macquarie University Human Research Ethics Committee. Written consent was obtained from participants aged 18 or over, or from a parent/guardian for participants aged under 1

\begin{tabular}{|c|c|c|c|c|c|c|}
\hline & $\underline{\mathbf{N}}$ & Age & $\underline{\text { Male/Female }}$ & $\begin{array}{c}\frac{\text { Raven }}{\text { Matrices }} \\
\text { Score } 160 \\
\end{array}$ & $\begin{array}{l}\text { Mind in the } \\
\text { Eyes Score } \\
\text { (\% Correct) }\end{array}$ & $\begin{array}{l}\text { Autism } \\
\text { Quotient } \\
\text { Score /50 } \\
\end{array}$ \\
\hline ASD & 16 & $\begin{array}{l}15.28 \\
(3.04)\end{array}$ & $\begin{array}{c}13 \text { male; } 3 \\
\text { female }\end{array}$ & $44.19(7.80)$ & $72.13(10.89)$ & $35.8(7.8)$ \\
\hline Control & 16 & $\begin{array}{l}15.23 \\
(3.10)\end{array}$ & $\begin{array}{c}13 \text { male; } 3 \\
\text { female }\end{array}$ & $47.63(8.08)$ & - & - \\
\hline
\end{tabular}

Table 5.1: Participant demographic and behavioural data.

\subsubsection{Experimental Paradigm and Design}

The auditory oddball paradigm was adapted from Recasens \& Uhlhaas (2017), and programmed in Psychopy v1.85 (Peirce, 2009). Stimuli were auditory tones consisting of superimposed sine waves $(440$ and $880 \mathrm{~Hz}$ ) synthesized with a $5 \mathrm{~ms}$ rise and fall time. Tones were presented at $\sim 70 \mathrm{~dB}$ via an MEG compatible $60^{*} 60 \mathrm{~cm}^{2}$ high quality speaker (Panphonics SSH sound shower, Panphonics), situated at the end of the participant bed. There were three trial types: "standard" trials in which 5 identical $80 \mathrm{~ms}$ tones were presented; "deviant" trials in which four identical $80 \mathrm{~ms}$ tones were presented and followed by a shorter $40 \mathrm{~ms}$ tone; and "omission" trials in which only four $80-\mathrm{ms}$ tones were presented, see Figure 5.1. Within each trial, tones were separated by a $0.15 \mathrm{~s}$ stimulus 
onset asynchrony (SOA) period. Between each trial, there was a silent interval which was presented with a randomised duration of between of 0.7 and $1.0 \mathrm{~s}$. The standard sequence was presented $60 \%$ (total 360 trials), the deviant sequence $20 \%$ (total 120 trials) and the omission sequence $20 \%$ (total 120 trials) of the time. Trials were presented in a pseudorandom order such that at least one standard sequence was presented after each deviant/omission trial. Furthermore, 3 standard sequences were presented at trial numbers 1-3, 201-203 and 401-403. As attention has been shown to affect auditory oddball response in ASD (Dunn et al., 2008), participants were instructed to ignore the auditory stimuli while watching a silent movie of their choice.

The experiment consisted of two blocks: one with no background noise; and the other with added background noise. Block presentation order was counter-balanced across participants. The background noise consisted of six-speaker babble noise, retaining the long-term spectrum and broadband modulations of human speech, see Table 1, Track 7 in (Dreschler et al., 2001). Such stimuli are perceived as white noise with spectrotemporal dips, and have previously been shown to affect auditory processing and MMF responses in ASD (Mamashli et al., 2017).

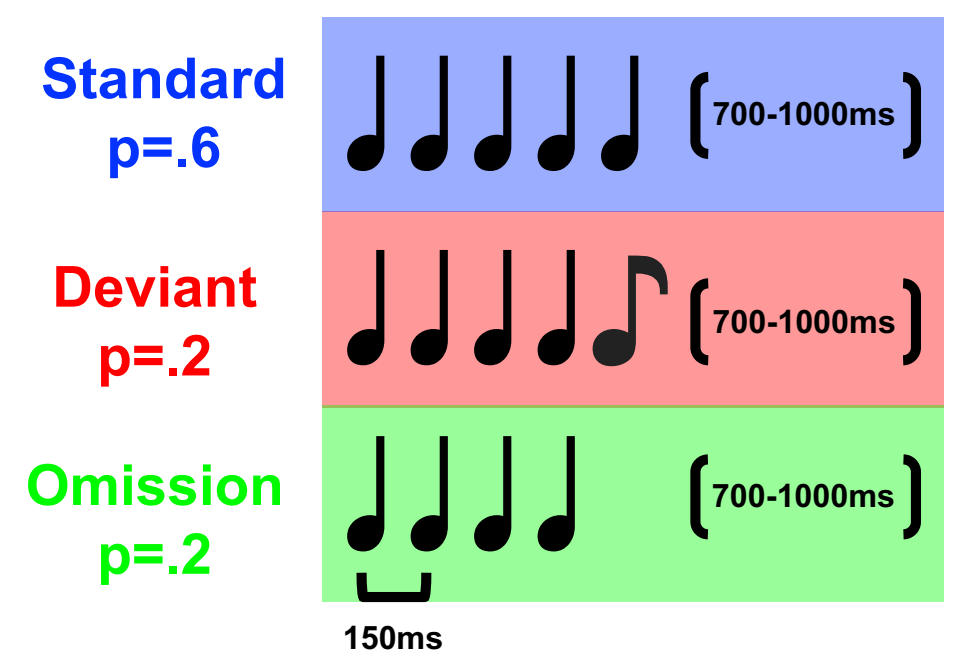

Figure 5.1: The auditory oddball paradigm used in this study, adapted from Recasens \& Uhlhaas (2017).

\subsubsection{MEG and Structural MRI Acquisition}

MEG data were acquired using the KIT-Macquarie MEG160 scanner (Model PQ1160RN2, KIT, Kanazawa, Japan), at a sampling rate of $1000 \mathrm{~Hz}$ and with an online bandpass filter of $0.03-200 \mathrm{~Hz}$. The recordings consisted of 160 coaxial first-order gradiometers with a $50 \mathrm{~mm}$ baseline (Kado et al., 1999). MEG data were acquired in a magnetically shielded 
room (Fujihara Co. Ltd., Tokyo, Japan) with participants lying in a supine position. Five head position indicator or "marker" coils were applied for head position tracking, with measurements taken before and after each experimental block (no-noise and noise). For MEG-MRI coregistration purposes, three anatomical landmarks (nasion, left pre-auricular, right pre-auricular), the locations of the marker coils and 1000-5000 points from the head surface were acquired using the integrated Polhemus Fastrak digitizer. Visual stimuli were presented on a projection screen located approximately $120 \mathrm{~cm}$ from participants. Data acquisition was divided into two separate blocks (tones presented with, or without background noise), each of which lasted approximately 15 minutes.

A structural T1 MPRAGE whole-head scan was acquired for the purposes of source reconstruction using a Siemens MAGNETOM Trio 3T scanner with a 32-channel head coil ( $T E=3.61 \mathrm{~ms}, T R=2000 \mathrm{~ms}, \mathrm{Tl}=900 \mathrm{~ms}$, flip angle $=9^{\circ}$, voxel-size $=1.0 \times 1.0 \times 1.0 \mathrm{~cm}$ ).

\subsubsection{MEG Preprocessing}

Data pre-processing was performed using the Fieldtrip toolbox v20170501 (Oostenveld, Fries, Maris, \& Schoffelen, 2010). For each participant, the entire recording was bandpass filtered between $0.5-250 \mathrm{~Hz}$ (Butterworth filter) and band-stop filtered to remove residual $50 \mathrm{~Hz}$ power-line contamination and its harmonics. So that the analysis was based on trials where a robust prediction of the regularity of auditory tones could be assumed to have been established, the first 100 trials were excluded. Data were then epoched, based on the onset of the first tone of a sequence, into segments of $1.6 \mathrm{~s}(0.3 \mathrm{~s}$ pre, $1.3 \mathrm{~s}$ post stimulus onset), and each trial was demeaned and detrended. Trials containing artefacts (SQUID jumps, eye-blinks, head movement) were removed by visual inspection. Per participant, there was an average of 98.9 omission trials and 99.0 deviant trials. To ensure there was a similar number of standard trials compared with deviant/omission trials, 99 standard trials were randomly selected from the cleaned data. Seven MEG channels containing large amounts of non-physiological noise were removed from all source-level analyses (channels: 024,081,085,089,095,149,150).

\subsubsection{MEG-MRI Coregistration}

MEG data were co-registered with participants' T1 MRI structural scan by matching the digitised head shape data with surface data from the structural scan (Jenkinson \& Smith, 2001). Subsequently, the aligned MRI-MEG image was used to create (i) a forward model based on a single-shell description of the inner surface of the skull (Nolte, 2003), using the segmentation function in SPM8 and (ii) spatial normalisation parameters to create individual volumetric grids. To facilitate group analysis, each individual volumetric grid was 
warped to a template based on the MNI brain ( $8 \mathrm{~mm}$ resolution). Subsequently the inverse of the normalisation parameters were applied to the template grid, for source analysis.

\subsubsection{Sensor Level Analysis}

As per Recasens \& Uhlhaas (2017), trials were bandpass filtered between 1-30 Hz (twopass Butterworth) and baseline corrected using $0.3 \mathrm{~s}$ before the onset of the first sound in the sequence. Sensor-level event-related fields (ERFs) were then calculated using the Fieldtrip toolbox (Oostenveld et al., 2010). Planar gradient transformations of the axialgradiometer data were calculated for each MEG sensor, by calculating the average of the absolute values of the first spatial derivatives in two orthogonal directions (Bastiaansen \& Knösche, 2000). This reduces the dimensionality of the data, making interpretation at the sensor-level easier. The duration MMF, durMMF, was calculated by subtracting deviant from standard ERFs; and omission MMF, omiMMF, by subtracting omission from standard ERFs. Latencies of durMMF and omiMMF are expressed relative to the onset of the duration mismatch $(0.65 \mathrm{~s})$ and tone omission $(0.6 \mathrm{~s})$, rather than to the onset of the sound sequence.

For each participant, ERFs were averaged from 17 sensors of interest, see Figure 5.3F. The latency of durMMF and omiMMF responses identified on a subject-by-subject basis (between 0-0.25s durMMF and 0-0.3s omiMMF), using the MATLAB function findpeaks to quantify the maximum ERF peak. Individual durMMF and omiMMF peaks are plotted in Appendix 3.

\subsubsection{MEG Source-Level}

Source analysis was conducted using a linearly constrained minimum variance beamformer (Van Veen, van Drongelen, Yuchtman, \& Suzuki, 1997), which applies a spatial filter to the MEG data at each vertex of the cortical mesh. To create a 'common' filter, covariance matrices were constructed using data from $0-0.3$ s following the onset of the final tone. Matrices from each of the three conditions were then averaged.

Beamformer weights were calculated by combining this matrix with leadfield information. Due to rank reduction, a regularisation parameter of lambda $5 \%$ was applied during beamforming. For durMMF and omiMMF responses, source localisation was performed using $0.1 \mathrm{~s}$ of data, centred on the peak MMF response, defined on a subject-by-subject basis at the sensor level, see above.

Six regions of interest (bilateral primary auditory cortex, A1, superior temporal gyrus, STG, and inferior frontal gyrus, IFG) were defined based on the underlying sources of MMN/F 
responses, according to previously published results (Chennu et al., 2016; Garrido et al., 2008, 2009). Specific MNI coordinates are shown in Table 5.2. The whole-brain results, see Figure 5.4, showed spatial MMF responses which overlapping with bilateral $A 1$ and STG.

\section{Source Location (MNI)}

\begin{tabular}{ccc} 
Source Name & Left Hemisphere & Right Hemisphere \\
\hline A1 & $-42-227$ & $46-148$ \\
STG & $-61-328$ & $59-258$ \\
IFG & -46208 & 46208 \\
\hline
\end{tabular}

Table 5.2 MNI coordinates of the regions of interest.

\subsubsection{Intertrial Coherence}

For omission trials, inter-trial coherence (ITC) was calculated for each ROI, from 0-0.3s following the omission of the fifth tone. ITC is a measure of band-limited phase consistency across trials: a value of 0 , indicates complete absence of phase consistency; whereas a value of 1 indicates perfect phase consistency across trials. At each time $t$ and

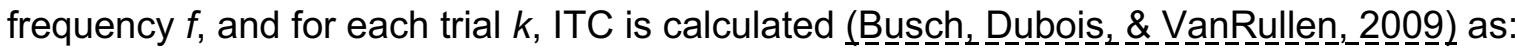

$$
I T C_{t, f}=\frac{1}{k} \sum_{n=1}^{k} e^{-i \varphi k(t, f),}
$$

Phase information was quantified using a 0.25 s sliding window (step size $0.02 \mathrm{~s}$ ), from 1$30 \mathrm{~Hz}$. Statistical comparison between groups was performed using cluster-based permutation testing, see Statistical Analysis.

\subsubsection{Granger Causality Analysis}

Directed functional connectivity between the ROls was quantified using spectrallyresolved non-parametric Granger causality (Dhamala, Rangarajan, \& Ding, 2008) as implemented in the Fieldtrip toolbox (Oostenveld et al., 2010). Data from 0-0.3s following the onset of the final tone was Fourier transformed (Hanning taper; $2 \mathrm{~Hz}$ spectral smoothing; 4s zero-padding), and entered into a non-parametric spectral matrix 
factorisation procedure. Granger causality was then estimated between each ROI pair and each ROI-scrambled time-series. Across hemispheres, corresponding ROI were averaged pairs (e.g. left STG-IFG and right STG-IFG). Statistical comparison between conditions was performed using cluster-based permutation testing, see 5.3.10 Statistical Analysis.

\subsubsection{Statistical Analysis}

For MEG data, statistical analysis was performed using cluster-based permutation tests (Maris \& Oostenveld, 2007), which consist of two parts: first an independent-samples ttest is performed, and values exceeding an uncorrected significance threshold of $p<.05$ are grouped into clusters. The maximum t-value within each cluster is carried forward. Second, a null distribution is obtained by randomising the condition label (e.g. ASD/control; deviant/standard; omission/standard) 4000 times and calculating the largest cluster-level t-value for each permutation. The maximum $t$-value within each original cluster is then compared against this null distribution, and the null hypothesis is rejected if the test statistic exceeds a threshold of $p<.05$.

Additionally, p-values were corrected for multiple comparisons across the six ROls using false-discovery rate correction (Benjamini \& Hochberg, 1995).

\subsection{Results}

\subsubsection{Sensor-Level Results}

To investigate the data at the sensor-level, event-related fields (ERFs) were computed for a group of right and left hemisphere sensors overlapping with the approximate location of auditory cortex, see Figure 5.2F. Following the final tone, presented at $0.6 \mathrm{~s}$, differences emerge for both control and ASD groups. The durMMF, plotted in black Figure 5.2A-B, increased from $0.75-0.9 \mathrm{~s}$ for the control group, and 0.8-1.0s for the ASD group. The omiMMF, plotted in yellow Figure 5.2A-B, showed a small peak around $0.65 \mathrm{~s}$ for controls, before decreasing from $0.7-0.75 \mathrm{~s}$ across both groups, and then increasing from 0.75 $0.85 \mathrm{~s}$. The results from (Recasens \& Uhlhaas, 2017), Figure 5.2C, show a very similar pattern of ERF activity, with a slightly more pronounced durMMF (black line), potentially due to the use of headphones rather than speakers. Henceforth, latencies of durMMF and omiMMF responses will be expressed relative to the onset of the duration mismatch $(0.65 \mathrm{~s})$ and tone omission (0.6s), see Figure 5.2D-E. MMF responses were defined on a subject-by-subject basis, see Methods \& Materials and Appendix Figure 3. 
The sensor-level analysis was repeated for the noise block, in which tones were presented in babble-noise (Dreschler et al., 2001). However both groups failed to show reliable durMMF or omiMMF responses, see Figure 5.3A-B. Subsequent analyses were therefore constrained to the no-noise block. 
A

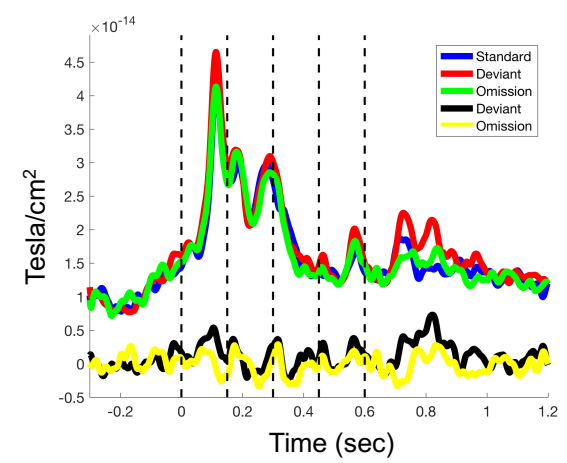

D

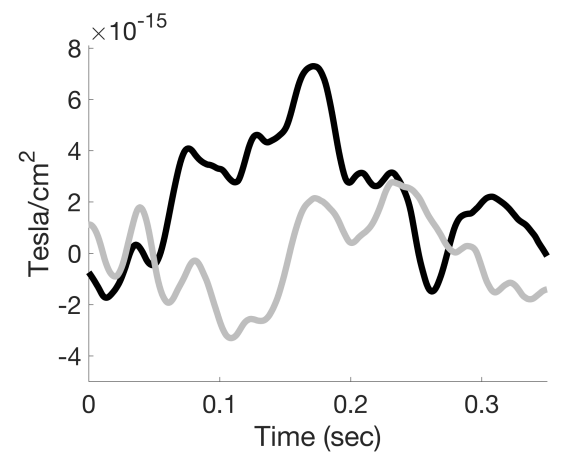

B

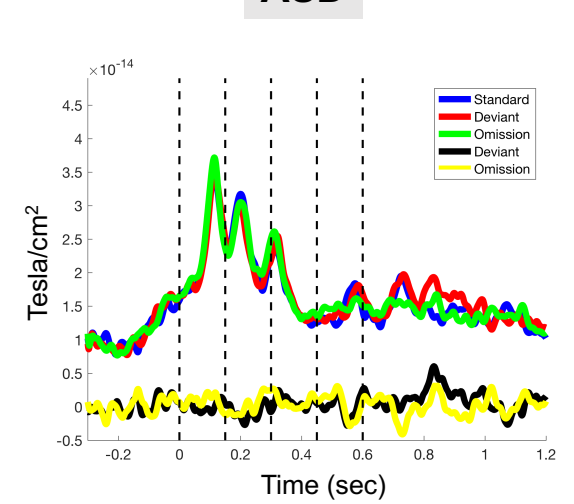

E

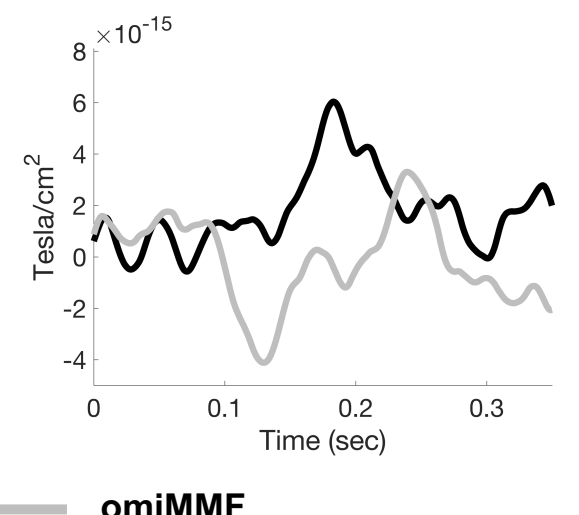

C

Recasens \& Uhlhaas, 2017

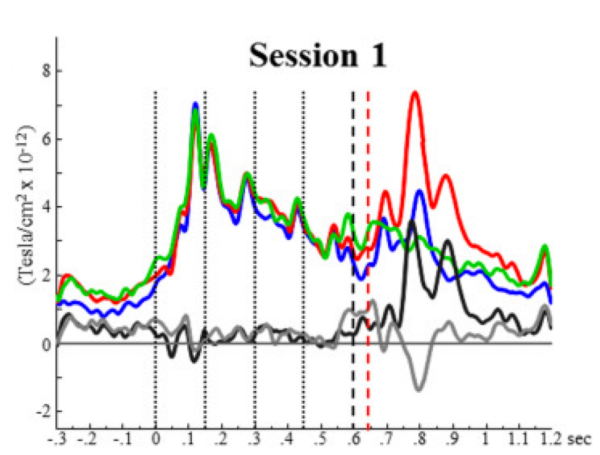

$\mathbf{F}$

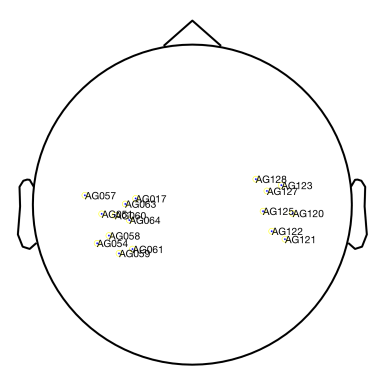

Figure 5.2 Sensor-Level Results (No noise block). (A-B) Evoked Fields were averaged over 17 MEG sensors. Dotted lines represent tone presentations. (C) Results from Recasens \& Uhlhaas (2017). (D-E) durMMF and omiMMF responses expressed relative to deviant onset.

(F) Location of the sensors of interest. 
A

\section{Control}

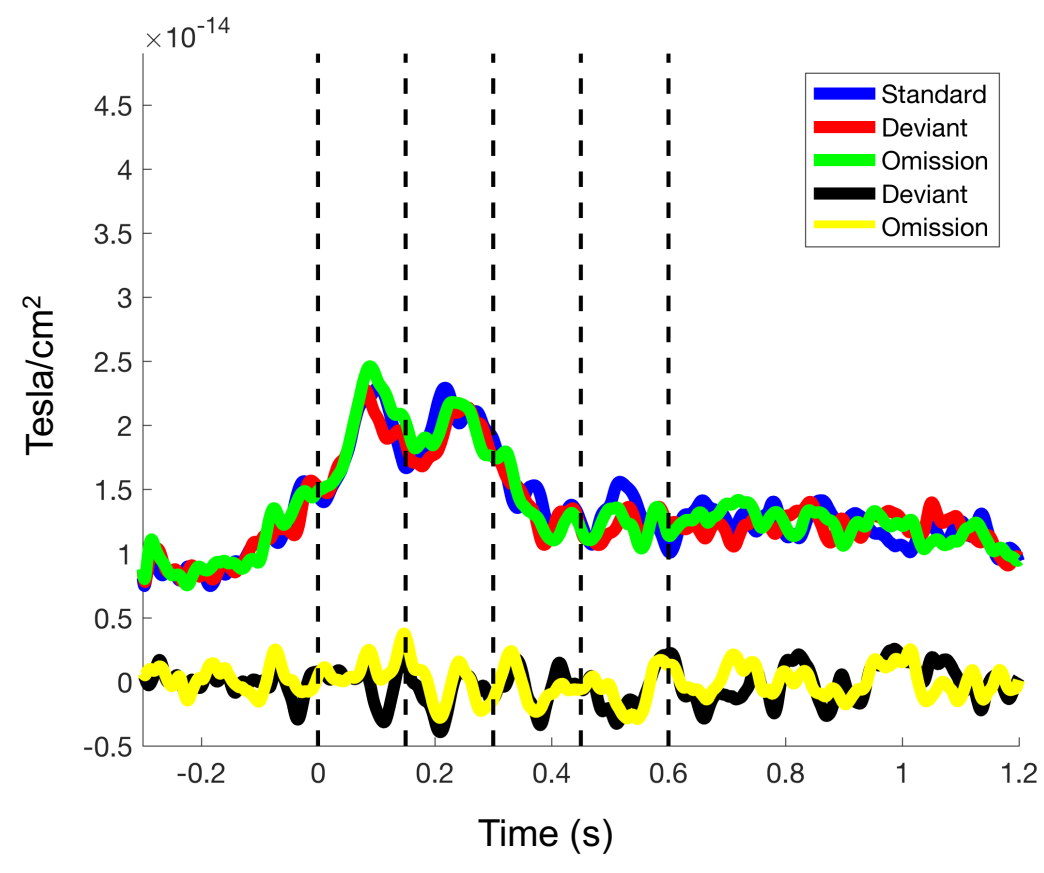

B

\section{ASD}

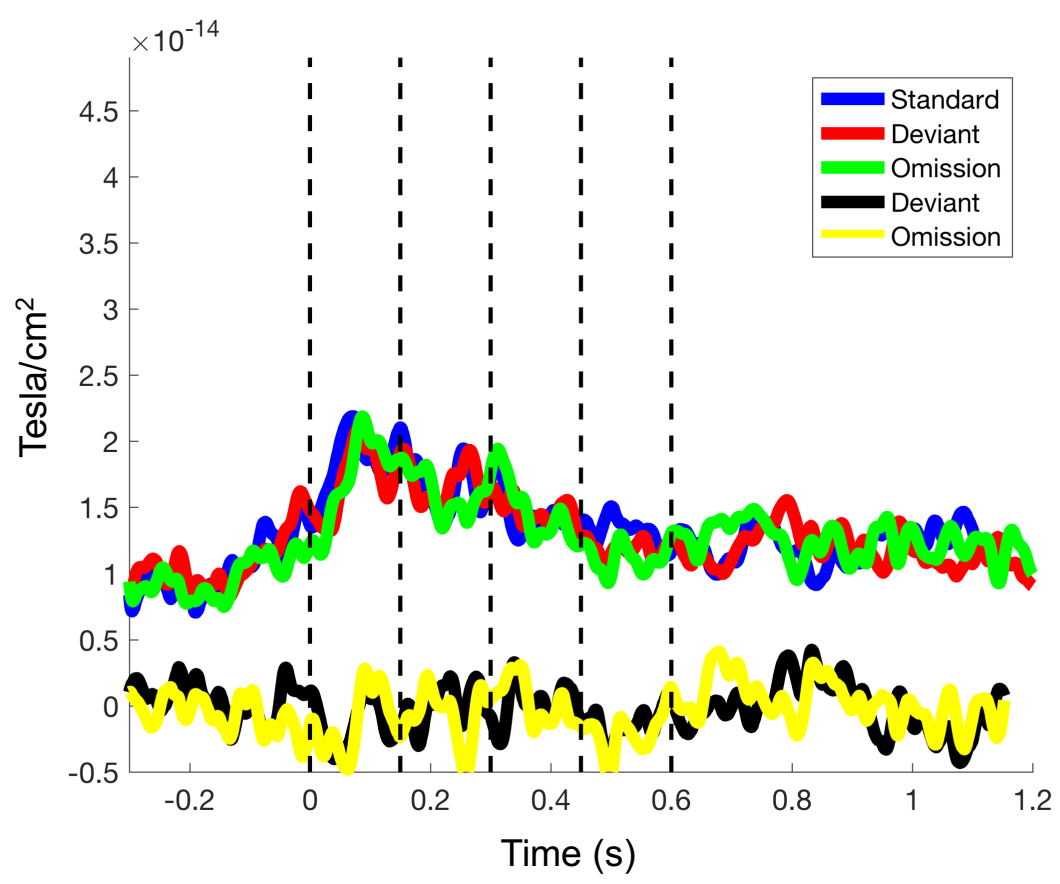

Figure 5.3 Sensor-Level Results (Noise block). (A-B) Evoked Fields were averaged over 17 MEG sensors. Dotted lines represent tone presentations. 


\subsubsection{Source-Level Results}

MMF responses were localised at the source level using an LCMV beamformer, see Methods and Materials. For both groups, the changes in power $(1-30 \mathrm{~Hz})$ was statistically compared between standard and deviant conditions (durMMF) and omission and standard conditions (omiMMF). Whole-brain maps were thresholded at $p<.05$, corrected for multiple comparison using cluster-permutation statistics (Maris \& Oostenveld, 2007). For the control group, the durMMF and omiMMF responses were accompanied by a significant, $p<.05$, increase in power for right parieto-temporal cortex overlapping with the superiortemporal gyrus and Heschl's gyrus (primary auditory cortex), Figure 5.4A. These regions are implicated in early auditory processing. For the ASD group, the durMMF was accompanied by a significant, $p<.05$, increase in power for right as well as left temporoparietal cortex, overlapping with the superior-temporal gyrus and Heschl's gyrus (primary auditory cortex). The omiMMF for the ASD group localised to a more extended set of frontal and temporal regions, Figure 5.4B.

In order to investigate MMF responses and group differences further, regions of interest were defined in bilateral primary auditory cortex, A1, superior temporal gyrus, STG, and inferior frontal gyrus, IFG). Co-ordinates for ROls were defined based on the underlying sources of MMN/F responses, according to the previous literature (Chennu et al., 2016; Garrido et al., 2008, 2009), see Table 5.2. 

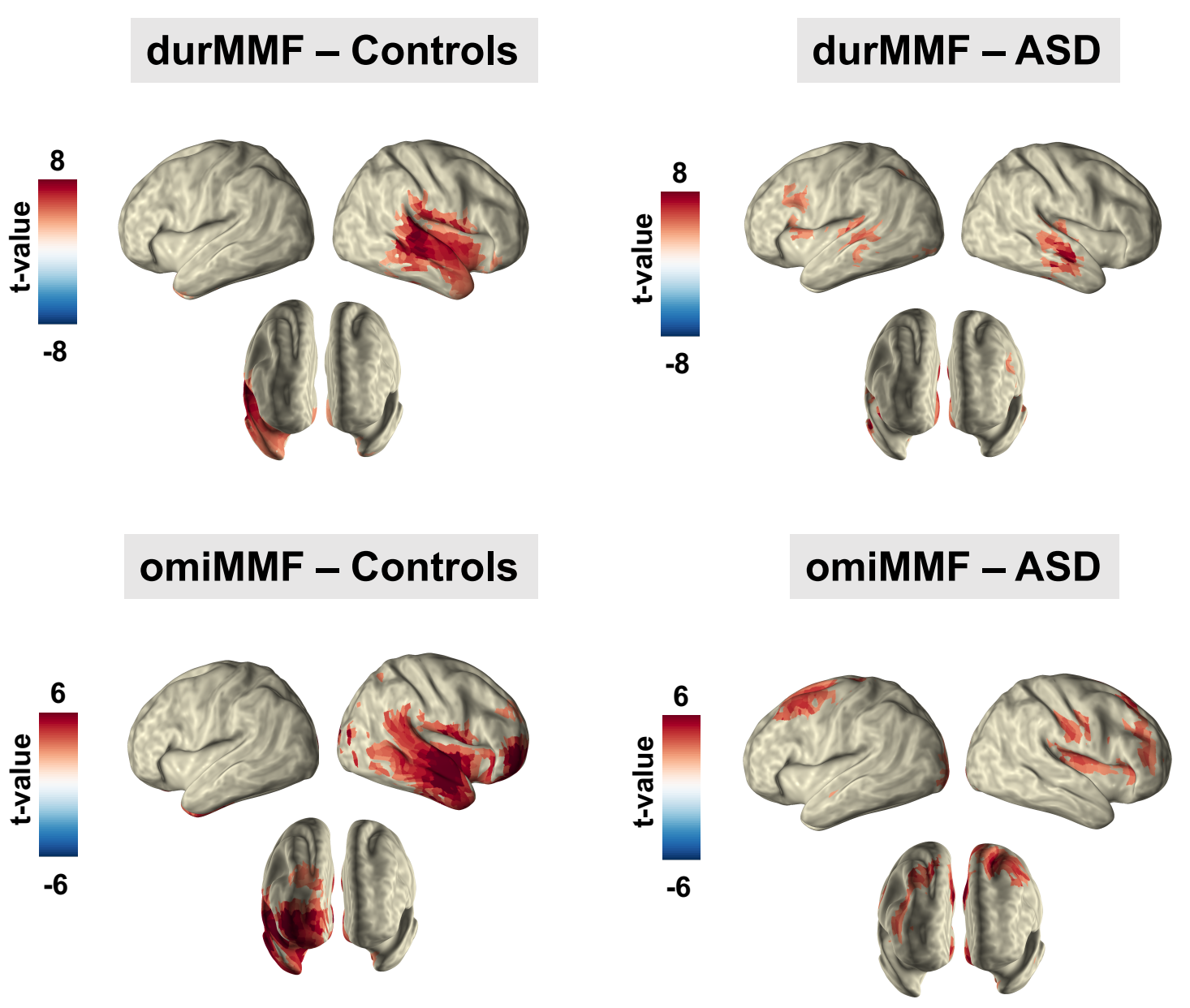

Figure 5.4 Source-Level Results. Whole-brain power maps were projected onto an inflated cortical surface (Caret), and masked at p<.05, corrected for multiple comparison. 


\subsubsection{Region of Interest ERF Results}

For each ROI, durMMF and omiMMF responses were computed, Figure 5.5A-B, ASD ERFs plotted in green, controls in purple. There were large durMMF and omiMMF responses in right STG and right $A 1$, as well as left STG for the omiMMF. Statistical comparison of the groups showed that controls had larger omiMMF responses in left STG, 0.088-0.107s, $p=.044$; and right STG, 0.065-0.098s, $p=.018$, Figure 5.5C. No significant group differences were found for the durMMF, $p>05$.

\subsubsection{Region of Interest Inter-trial Coherence Results}

In order to estimate the frequency band(s) underlying the ERF group differences, inter-trial coherence (ITC) was calculated for each ROI, from $1-30 \mathrm{~Hz}$. We concentrated specifically on omission trials for a time-period $0-0.3$ following the omitted tone. Across groups, results show increases in ITC primarily for the delta $(2-4 \mathrm{~Hz})$, theta $(4-8 \mathrm{~Hz})$ and low-alpha $(8-10 \mathrm{~Hz})$ bands, within left/right A1 and STG, Figure 5.6A-B. Whilst ITC values were generally higher for the controls, statistical comparisons between groups revealed no effects, $p>.05$, corrected for multiple comparisons across time, frequency or ROI, Figure $5.6 \mathrm{C}$.

\subsubsection{Granger Causality}

Finally, directed functional connectivity was computed between each of the ROIs using non-parametric spectrally resolved granger causality (GC). Corresponding ROI pairs were averaged across hemispheres. Across groups, there were no GC spectral differences between conditions, $p<.05$, corrected for multiple comparisons, Figure 5.7. 
A

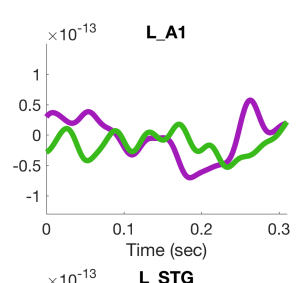

$\times 10^{-13} \quad$ Time $(\mathrm{sec})$

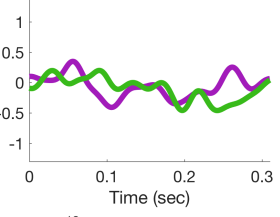

$\times 10^{-13} \quad$ Time (sec)

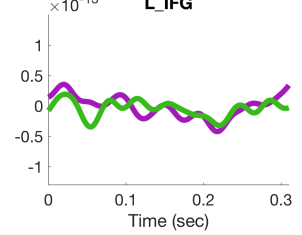

B
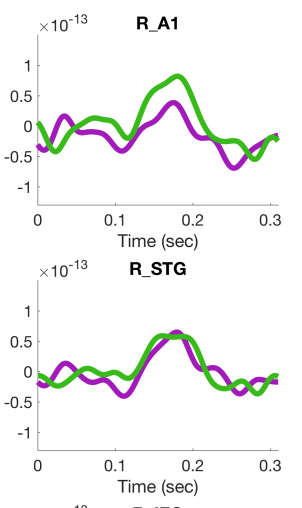

$\times 10^{-13} \quad$ R IFG

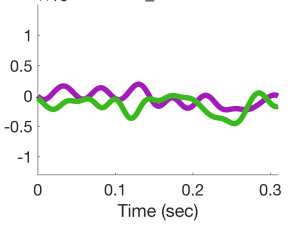

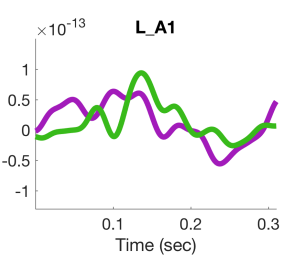

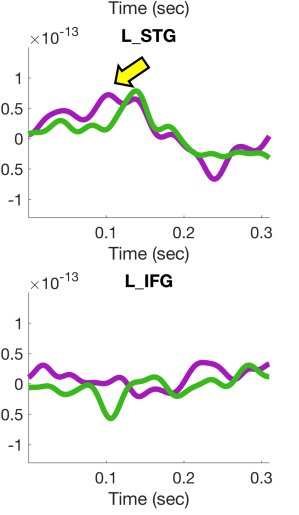

Control

\section{omiMMF}
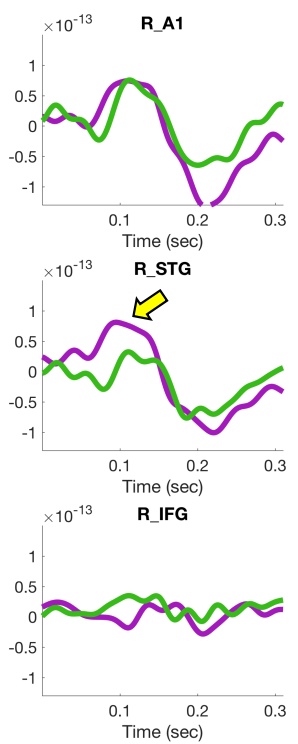

0.2
$(\sec )$
C Controls>ASD

omiMMF: Right STG

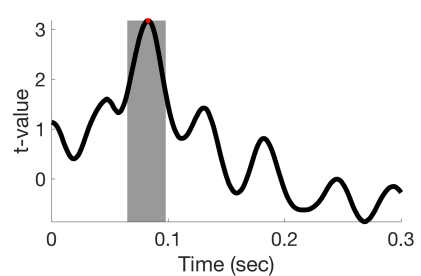

omiMMF: Left STG

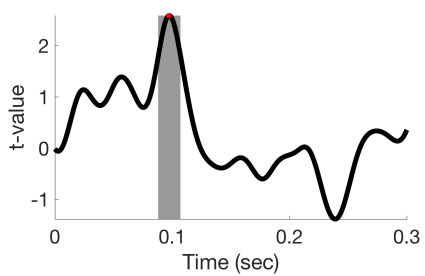

No other significant clusters $(p<.05)$

Figure 5.5 Region of Interest Event-Related Field (ERF) Results. (A) durMMN and (B) omiMMF ERFs are shown for each of the six regions of interest. Controls are plotted in purple, whereas ASD data is plotted in green. Yellow arrows indicate regions of interest with significant group differences, $p<.05$. (C) Significant group differences in the omiMMF are expressed in terms of $t$-values with significant clusters shaded in grey. 
A

\section{Omission Controls}
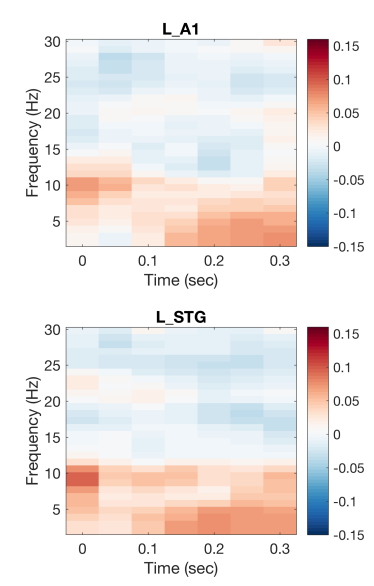

LIFG
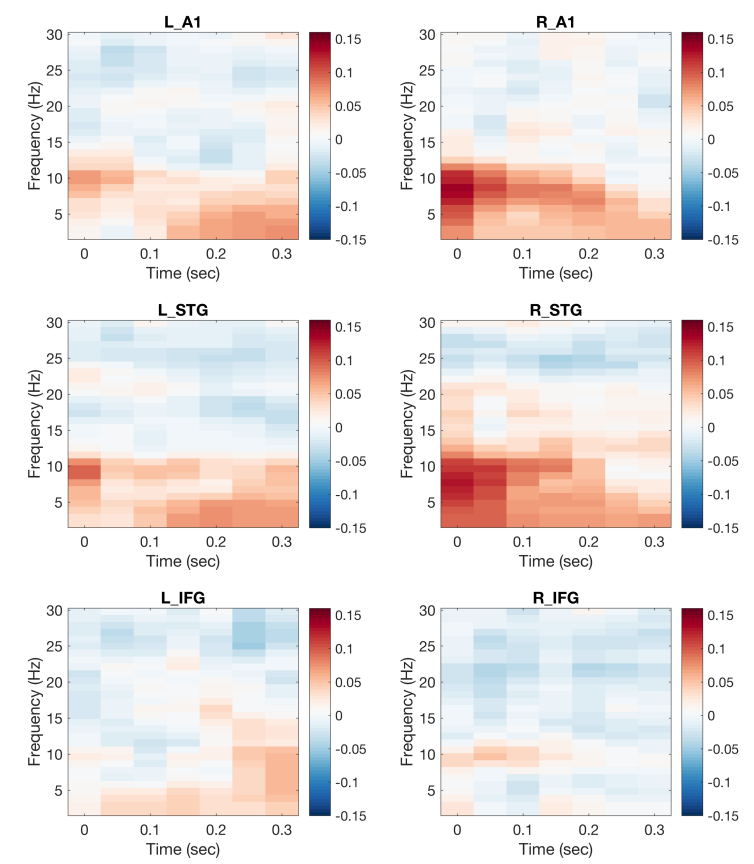

B
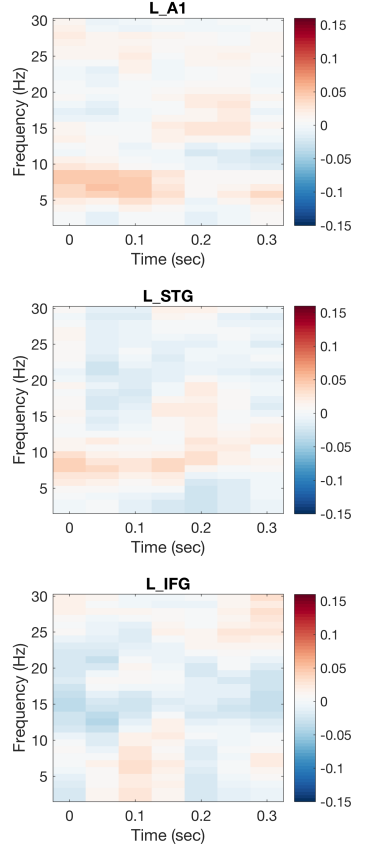

Omission ASD
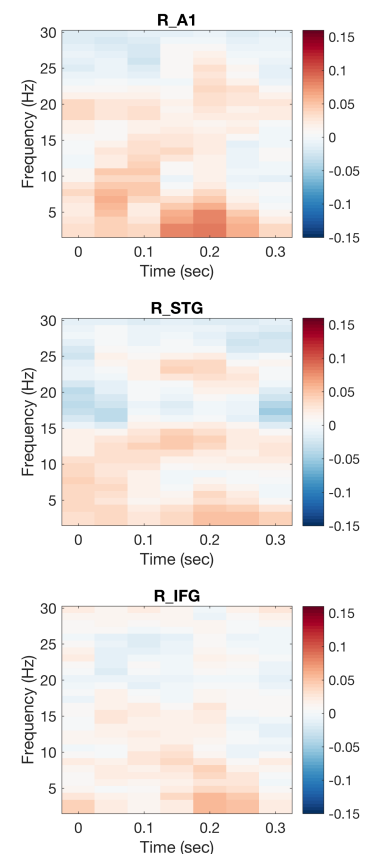

C

\section{Omission Controls> ASD}
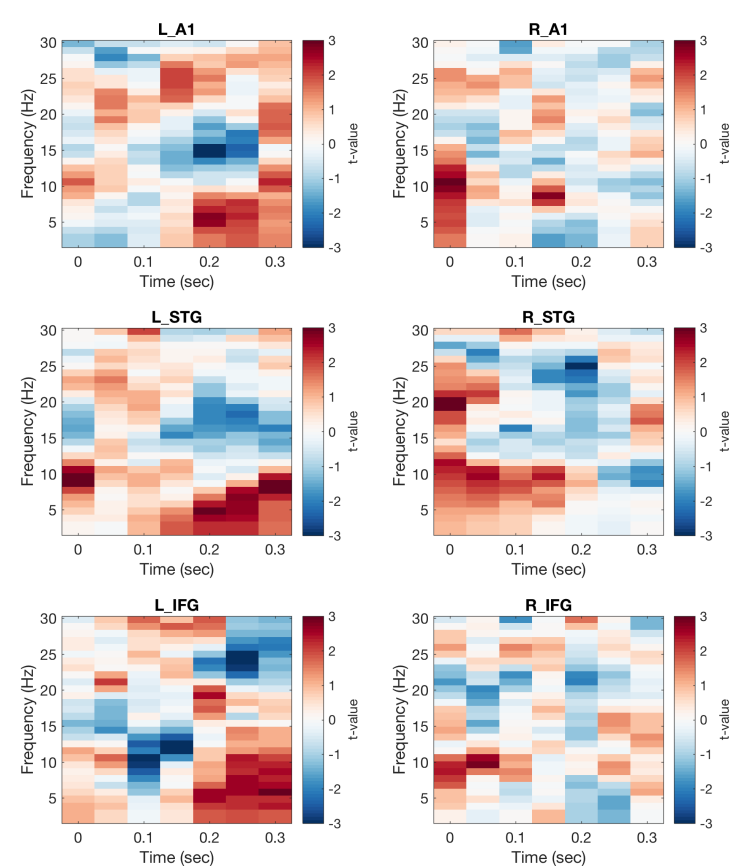

No significant clusters $(p<.05)$

Figure 5.6 Region of Interest Intertrial Coherence (ITC) Results. ITC was calculated for omission trials from 0-0.3s following the omission of the tone. Data from (A) Controls and (B) ASD groups are shown separately. (C) Statistically comparing ITC values across groups, there were no significant differences, $p<.05$, over frequency and time. 
A
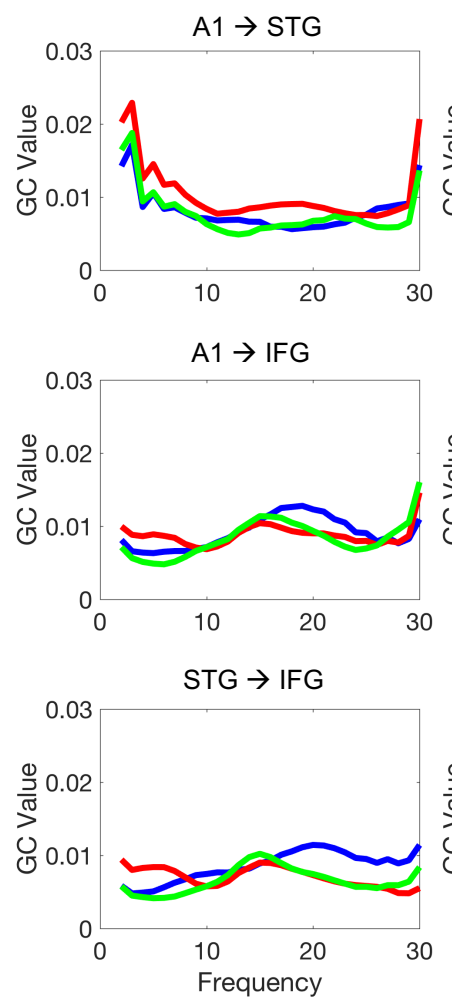

\section{Controls}
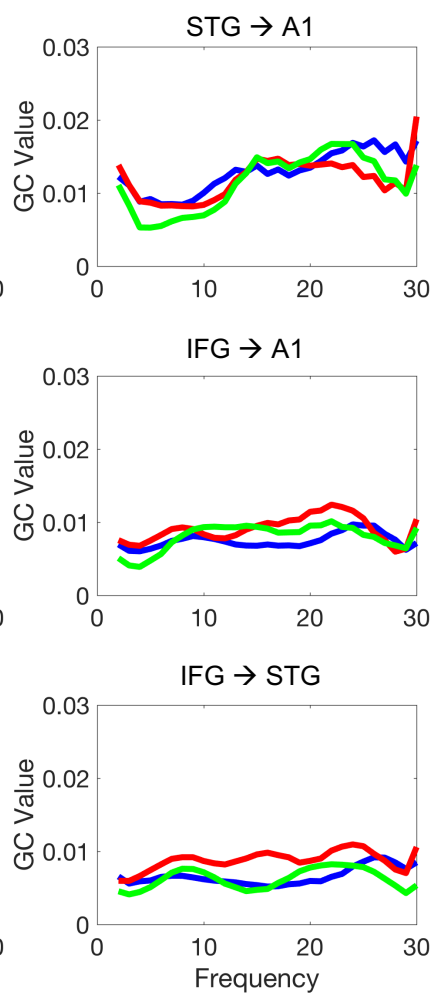

B
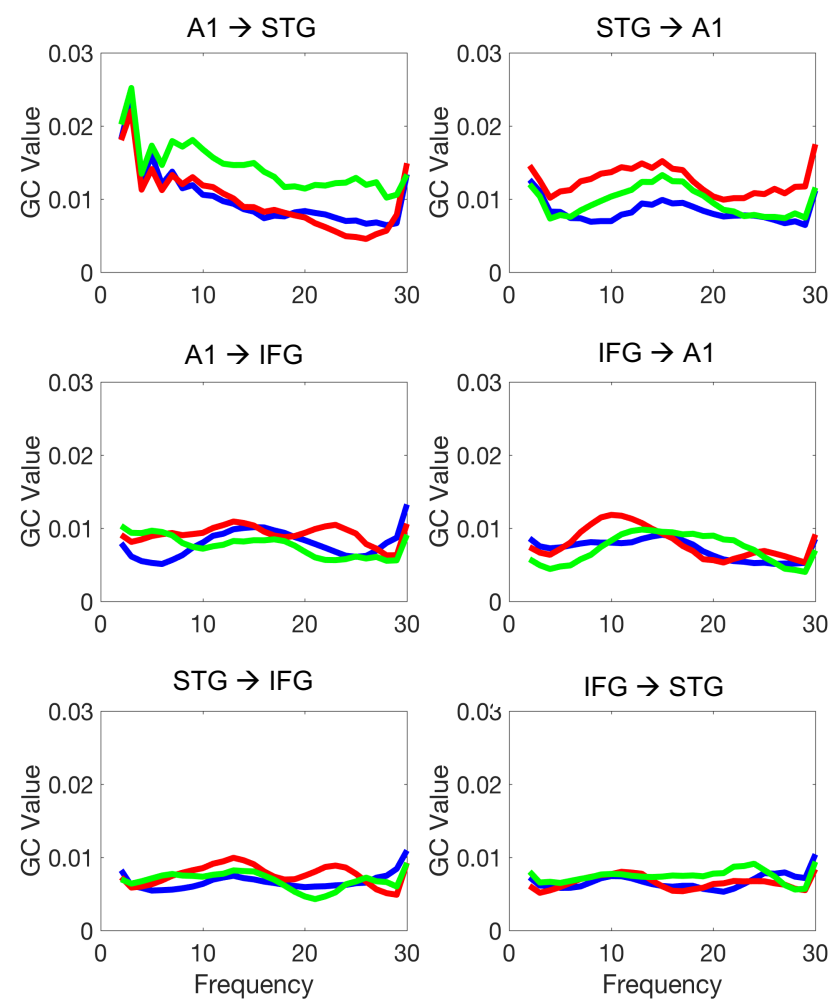

\section{ASD}

$$
\text { ( }
$$




\subsection{Discussion}

This study investigated predictive-coding in ASD using a passive auditory oddball paradigm. When an expected tone was omitted, the ASD group showed a reduced response in bilateral superior temporal gyrus (STG) compared to controls. When an unexpected duration deviant was presented, no group differences in the MMF response, durMMF, were found. We also presented auditory tones in babble noise, with the goal of investigating mechanisms of precision-weighting in ASD and control groups. However, this manipulation removed both omiMMF and durMMF responses across groups.

Our results support predictive coding accounts of ASD, which focus on "hypo-priors" or "reduced top-down predictions" (Pellicano \& Burr, 2012; Van Boxtel \& Lu, 2013). The reduced omiMMF within STG implies that the ASD group generated weaker top-down predictions about the final tone in the sequence, resulting in a reduced oddball response. The effect occurred at very early latencies after tone omission, around $0.1 \mathrm{~s}$. This suggests that ASD-related differences occur at the level of low-level prediction violations, i.e. MMN/F responses, rather than higher-level prediction-violations, reflected in the P300 evoked potential/field (Wacongne et al., 2011). Very early omission-related responses are also reported by other studies (Hughes et al., 2001; Recasens \& Uhlhaas, 2017; SanMiguel, Widmann, Bendixen, Trujillo-Barreto, \& Schröger, 2013; Yabe, Tervaniemi, Reinikainen, \& Näätänen, 1997). By analysing inter-trial coherence in six regions of interest, this chapter has reported that following tone omission, phase synchrony increased within the $\mathrm{A} 1$ and STG for low frequencies, from $2-10 \mathrm{~Hz}$. This is consistent with previous studies which have investigated the oscillatory basis of oddball responses (Recasens, Gross, \& Uhlhaas, 2018; Stothart \& Kazanina, 2013). However, we did not find any group differences in ITC values, perhaps due to the stringent statistical correction needed for correcting $p$-values over frequency, time and ROI .

Unfortunately, the noise manipulation used in this study resulted in the extinction of MMF responses in both control and ASD groups, and we were therefore unable to investigate precision-weighting theories of ASD (Lawson et al., 2014; Palmer et al., 2017). Using the same babble noise, but different oddball paradigm, (Mamashli et al., 2017) showed that MMF responses were still detectable in controls. This discrepancy could be due to differences between the oddball paradigms, as well as the MEG-compatible speakers used in this study, which may have produced neural responses with lower signal-to-noise compared with headphones used in other studies ${ }^{*}$. In any case, precision-weighting

"Having said this, MEG-compatible speakers are more comfortable than headphones and increase participant compliance. This is especially important for MEG-ASD studies. 
theories of ASD would hypothesise that reductions in the MMF response are even greater in noisy environments for autistic participants (Lawson et al., 2014; Palmer et al., 2017). When it is harder to extract signal from noise, predictions from higher areas would be relied upon more heavily to resolve prediction errors lower in the hierachy (Friston, 2008). Therefore in noisy environments, ASD-related group differences in the MMF might extend to frontal regions, as reported by (Mamashli et al., 2017). To disambiguate different predictive-coding theories of ASD, future work using the auditory oddball paradigm should incorporate precision weighting into experimental design, perhaps by parametrically modulating the predictability of auditory sequences (Barascud, Pearce, Griffiths, Friston, \& Chait, 2016), and/or psychophysically adjusting the level of background noise (Hirsh \& Watson, 1996).

Interestingly, this study found reduced MMF in ASD for omitted tones, but not for MMF responses to an unexpected deviant tone (i.e. duration deviant). To our knowledge, this is the first study to report reduced omission responses in ASD. As explained earlier, deviant MMN/F response are partly driven by neural adaptation: repeated presentations of the standard tone result in adaptation effects within auditory cortex, with the presentation of a deviant tone resulting in greater neural activity by comparison (May \& Tiitinen, 2010). Whilst oddball responses also reflect prediction error (Farley, Quirk, Doherty, \& Christian, 2010; Wacongne, Changeux, \& Dehaene, 2012; Wacongne et al., 2011), disentangling that from adaptation in traditional paradigms remains problematic (Garrido et al., 2009). For omission responses, because no tone is presented during the omission period, MMFs are unlikely to reflect the rebound from adaptation in auditory cortex, and therefore constitute a more reliable marker of prediction error (Recasens \& Uhlhaas, 2017; Sauer et al., 2017; Wacongne et al., 2011). Our results indicate that it is this neural process, rather than a simple adaptation, which is reduced in ASD. This may account for the variability within the auditory oddball ASD literature, as previous studies have focussed solely on deviant oddball stimuli rather than omissions.

Having said this, the precise relevance of omission responses to predictive-coding is still unresolved (Heilbron \& Chait, 2017). Some argue that omission responses purely reflect prediction error (SanMiguel et al., 2013) - that neural activity to unexpected silences, not driven by bottom-up sensory differences, must signal surprise. However, if prediction errors are calculated by subtracting predictions from sensory input (Friston, 2005), how can ommissions produce an increase in neural activity without negative firing rates (Wacongne et al., 2012)? Put another way, how can the brain generate something from nothing? Perhaps, omission responses, represent a secondary response, in which the 
brain transforms the prediction 'error' term into a neural signal which can be fed upwards through the hierarchy. In any case, omission responses represent a promising future research avenue for the study of predictive coding, especially as it relates to the neurobiology of ASD.

This study attempted to quantify the directed functional connectivity between key ROIs underlying the MMF, using non-parametric spectrally-resolved Granger causality (GC). However, no significant differences between conditions were found, for either ASD or control groups. This might be due to the fact that GC was calculated using data from 0$0.3 \mathrm{~s}$ following presentation/omission of the fifth auditory tone, in order to obtain enough data for spectral matrix factorisation (Dhamala et al., 2008). However, omission and deviant MMF effects were exceedingly transient, typically lasting only $0.1 \mathrm{~s}$. Therefore, by assuming stationarity, measures of GC may not be able to characterise short-lived patterns of connectivity during oddball responses. Furthermore, it has been shown that evoked neural responses can heavily bias measures of GC (Wang, Chen, \& Ding, 2008). Dynamic causal modelling may be a more appropriate approach in this context, having already been successfully used in the context of auditory oddball paradigms (Chennu et al., 2016; Garrido et al., 2008).

Finally, it is important to note that reductions in the auditory oddball response have been reported for other psychiatric conditions, most notably schizophrenia (Javitt, Lee, Kantrowitz, \& Martinez, 2018; Michie, 2001; Sauer et al., 2017), and Parkinson's Disease (Pekkonen, Jousmäki, Reinikainen, \& Partanen, 1995). Whilst still heavily debated, there is emerging evidence that predictive-coding represents a ubiquitous and fundamental computational mechanism of brain function (Bastos et al., 2012; Friston, 2005; Friston, 2017; Friston, Bastos, Pinotsis, \& Litvak, 2015). Therefore, it is not surprising that other psychiatric conditions show reduced oddball response: in theory, differences in brain connectivity, neurodegeneration, synaptic plasticity etc., could all result in alterations to predictive-coding mechanism(s). Finding the specific nature of ASD-related predictive coding deficits, perhaps from a developmental perspective, should be the focus of future research.

\section{Author Contributions}

RS, PFS, WH \& KK co-designed the study. RS collected the data, carried out the analysis and was the primary author of this chapter. 


\section{Conflict of Interests Statement}

The authors wish to declare the research was conducted in the absence of any commercial or financial relationships that could be construed as a potential conflict of interest.

\section{Acknowledgments}

We wish to thank: the volunteers who gave their time to participate in this study; Macquarie Medical Imaging for performing MRI scans; and Marc Recasens and Wouter Dreschler for providing auditory stimuli used for in the experimental paradigm. Robert Seymour was supported by a cotutelle PhD studentship from Aston University and Macquarie University. 


\section{Chapter 6: The Neural Basis of Perspective-Taking in Neurotypical and Autistic Populations}

\section{Seymour, R.A ${ }^{1,2,3}$., Rippon, $\mathbf{G}^{1}$., Gooding-Williams ${ }^{1}$, G,. Wang, $\mathrm{H}^{1}$., \& Kessler, $\mathrm{K}^{1}$.}

${ }^{1}$ Aston Brain Centre, School of Life and Health Sciences, Aston University, Birmingham, B4 7ET.

${ }^{2}$ Department of Cognitive Science, Macquarie University, Sydney, Australia, 2109.

${ }^{3}$ ARC Centre of Excellence in Cognition and Its Disorders, Macquarie University, Sydney, Australia, 2109.

Please note, Experiment 1 in this chapter has been published in a peer-reviewed journal:

Seymour, R. A., Wang, H., Rippon, G., \& Kessler, K. (2018). Oscillatory networks of highlevel mental alignment: A perspective-taking MEG study. Neurolmage, 177, 98-107.

\subsection{Abstract}

In this chapter, we investigated the neural basis of perspective-taking using magnetoencephalography. Recently Wang et al. (2016) showed that theta-band (3-7 Hz) brain oscillations within the right temporo-parietal junction (rTPJ) and brain regions coding for motor/body schema contribute to the process of perspective-taking. In Experiment 1, we set out to unravel the extended functional brain network in detail, collecting data from 18 neurotypical adults (i.e. healthy controls, no family history of ASD). Increasing the angle between self and other perspective was accompanied by longer reaction times and increases in theta power within rTPJ, right lateral prefrontal cortex (PFC) and right anterior cingulate cortex (ACC). Using Granger-causality, we showed that lateral PFC and ACC exert top-down influence over rTPJ, indicative of executive control processes required for 
managing conflicts between self and other perspectives. We also show that during perspective-taking, the rTPJ increases its theta-band phase synchrony with brain regions involved in mentalising and regions coding for motor/body schema. In Experiment 2, the same paradigm was used to investigate the neural basis of perspective-taking in a group of 18 adolescents diagnosed with ASD and 17 age-matched controls. Results showed that increasing the angle between self and other perspective was accompanied by longer reaction times for the ASD group, as well as reduced theta power in ventral visual cortex and lateral PFC. Additionally, using Granger causality we showed that, compared with controls, the ASD group had reduced theta-band connectivity from lateral PFC to visual area V5, as well as V1 to V5. On the other hand, the ASD group showed greater alpha desynchronisation over visual cortex compared with controls. These divergent theta/alpha effects were interpreted as evidence that ASD participants favour a mental rotation strategy, rather than an embodied strategy when asked to imagine the world from another's perspective.

\subsection{Introduction}

Humans possess highly developed social skills that allow us to imagine what others might be experiencing, thinking or feeling to an extent not shared by other species (Tomasello, Carpenter, Call, Behne, \& Moll, 2005). One important aspect of this is the ability to understand another's visuospatial experience of the world - a skill termed visual perspective taking (VPT) (Flavell, Everett, Croft, \& Flavell, 1981). In this study, we conducted two MEG experiments, which set out to: i) understand the patterns of oscillatory cortical connectivity underlying perspective-taking; and ii) investigate individual differences in the neurocognitive basis of perspective-taking between adolescents diagnosed with autism spectrum and age-matched control participants .

\subsubsection{Perspective-Taking versus Perspective Tracking}

Taking another's perspective can take one of two forms, see Figure 6.1. First, one can track another's view of the world ("can the other person see the object?"), sometimes termed level 1 perspective-taking, VPT-1. Second, one can understand how the world looks from another's point of view ("what does the object look like to the other person?"), also termed level 2 perspective-taking, VPT-2. These two processes have been related to different developmental stages, with perspective-tracking emerging around age 2, and perspective-taking emerging around ages 4-5 (Flavell et al., 1981; Gzesh \& Surber, 1985; Moll \& Tomasello, 2006). Furthermore perspective-tracking has been observed in other species such as apes and corvids (Bugnyar et al. 2004; Bräuer et al. 2007), whereas level 2 perspective-taking seems to be uniquely human (Surtees, Butterfill, \& Apperly, 2012). 


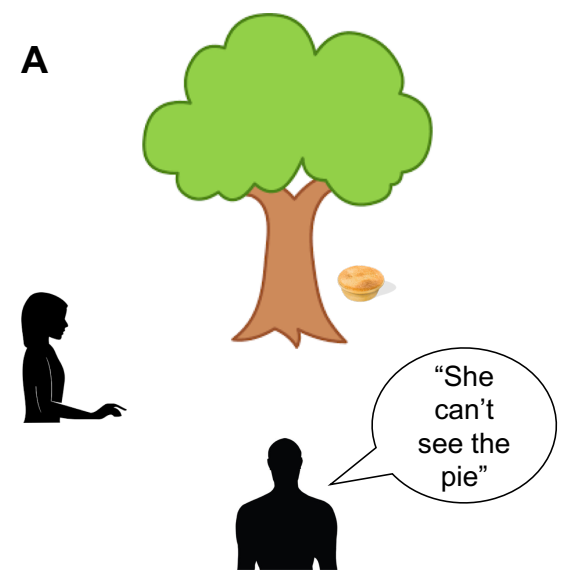

Perspective-Tracking

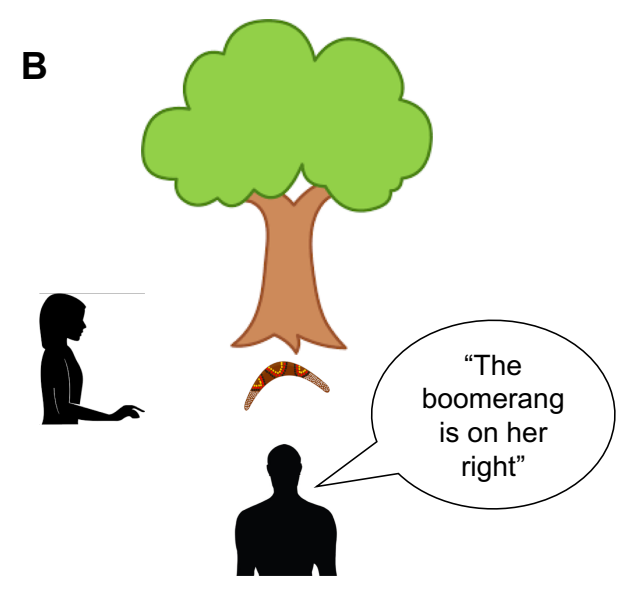

Perspective-Taking

Figure 6.1. Two real-life examples to demonstrate the difference between: $(A)$ perspective-tracking and $(B)$ perspective taking.

\subsubsection{Embodied Perspective-Taking}

There is growing evidence that perspective-taking is an embodied cognitive process, grounded in the internal bodily and action representations of the observer. Using posture manipulations, several studies (e.g. Gooding-Williams, Wang, \& Kessler, 2017; Klaus Kessler \& Rutherford, 2010; Klaus Kessler \& Thomson, 2010; Surtees, Apperly, \& Samson, 2013; Wang, Callaghan, Gooding-Williams, McAllister, \& Kessler, 2016) have shown that perspective-taking engages large parts of the neuronal bases of the body schema, i.e. the cortical correlates of the internal representation of the body (Coslett, Buxbaum, \& Schwoebel, 2008; Medina, Jax, \& Coslett, 2009). This suggests that highlevel perspective-taking involves the simulated rotation of the embodied self into another's orientation and perspective (Kessler \& Thomson, 2010; Surtees et al., 2013; Wang et al., 2016). In other words, humans literally rotate their own perspective to understand another's viewpoint. Interestingly, there are individual differences in the efficiency of this strategy, with East-Asian participants faster than Western participants, potentially reflecting an egocentric bias in Western cultures (Kessler, Cao, O'Shea, \& Wang, 2014).

\subsubsection{The Neural Correlates of Embodied Perspective-Taking}

There has been recent progress in characterising the neural correlates of embodied perspective-taking. In particular, the posterior division of the temporo-parietal junction (TPJ) (Igelström, Webb and Graziano 2015; Bzdok et al. 2013), seems to play a key role 
in perspective-taking, see meta-analyses from (Van Overwalle, 2009, 2011), and more generally for "mentalising" (representing other's mental states) (Schurz, Aichhorn, Martin, \& Perner, 2013; Van Overwalle, 2011). Using MEG, Wang et al. (2016) investigated embodied simulation during perspective-taking. Increases in low-frequency theta-band oscillations $(3-7 \mathrm{~Hz})$ were found for both the cognitive effort of perspective-taking (amount of angular disparity between self vs. other's viewpoint) and for embodied processing (posture congruence) during perspective-taking. These effects localised to the right pTPJ. Wang et al., (2016) also showed that disruptive dual-pulse TMS administered over pTPJ decreased RT during perspective-taking. In a follow-up study, Gooding-Williams et al. (2017) used repetitive TMS entrainment over pTPJ to show that TMS pulses administered at theta frequency $(6 \mathrm{~Hz})$ accelerated perspective-taking, while alpha $(10 \mathrm{~Hz})$ entrainment slowed perspective-taking down. Theta may therefore be the crucial neural frequency to facilitate brain connectivity between the TPJ and other brain regions crucial for perspective-taking.

More generally, the TPJ is implicated in a range of cognitive operations, including spatial attention, social cognition and self/other distinctions (Eddy, 2016; Geng \& Vossel, 2013). It has been suggested that the region acts as a major hub for information integration (Igelström and Graziano 2017.; Eddy 2016), especially during higher-level cognitive processes relying upon internal representations, such as perspective-taking (Igelström and Graziano 2017.; Eddy 2016; Wang et al. 2016; Gooding-Williams et al. 2017). Indeed, the TPJ has extensive functional connectivity to many networks of the brain, including the fronto-parietal control (Vincent, Kahn, Snyder, Raichle, \& Buckner, 2008), default mode (Mars et al., 2012), and ventral attention networks (Bzdok et al., 2013). We therefore hypothesised that the TPJ contributes to the process of embodied transformation through changes in patterns of whole-brain functional connectivity, via theta-band synchrony, as would be predicted from the region's role as a network hub (Igelström and Graziano 2017.; Carter and Huettel 2013; Eddy 2016). However, investigations of perspectivetaking using connectivity analysis, e.g. in form of frequency-specific phase-coupling, are scarce. To our knowledge, only one study to date has reported enhanced theta phasecoherence between right TPJ and ventromedial prefrontal cortex (VMPFC) in a condition that required participants to imagine another's visual experience (Bögels, Barr, Garrod, \& Kessler, 2015). The major aim of Experiment 1, see Section 6.2.5 Hypotheses, was therefore to consolidate the crucial role of pTPJ theta oscillations in perspective-taking by means of advanced network analyses. 
In addition to the TPJ, Wang et al., (2016) reported increases in theta-band power for the lateral PFC during the cognitive effort of perspective-taking. Activity within this region during social cognition has been argued to reflect high-level reasoning and working memory processes recruited more generally during complex perspective-taking and mentalizing tasks (Van Overwalle, 2011). However, there is emerging evidence that frontal activity in lateral PFC but also in the anterior cingulate cortex (ACC) could play a more nuanced role in perspective-taking by managing the conflict between self and other perspectives (Bögels et al., 2015; Hartwright, Apperly, \& Hansen, 2015; Samson, Apperly, Kathirgamanathan, \& Humphreys, 2005). Indeed, McCleery et al. (2011) found a frontal evoked response from $800-1000$ ms during perspective-taking, which was dependent on whether self-perspective was consistent with an avatar's perspective. In terms of thetaoscillations, this could potentially manifest as a direct connection between lateral PFC and the core mentalizing network (Bögels et al., 2015) in TPJ and vmPFC (Van Overwalle, 2009, 2011), see Section 6.2.5 Hypotheses.

\subsubsection{Perspective-Taking in Autism Spectrum Disorder}

Recent research has shown that participants diagnosed with Autism Spectrum Disorder (ASD) have difficulties with embodied perspective-taking. ASD is a complex neurodevelopmental condition characterised by difficulties in social interaction, language, as well as repetitive behaviours (APA, 2013). Reviewing the literature, Pearson, Ropar, \& Hamilton (2013) suggest that ASD participants show a selective impairment for embodied level-2 perspective-taking, but not level-1 perspective tracking, though see Tan \& Harris (1991). It has also been argued that impaired embodied perspective-taking could underlie the theory of mind deficits more commonly associated with ASD (Hamilton, Brindley, \& Frith, 2009; Pearson, Marsh, Ropar, \& Hamilton, 2016; Surtees et al., 2013). One recent study has suggested rather than adopting an embodied egocentric strategy, ASD participants tend to use a mental rotation (MR) strategy during perspective-taking (Pearson et al., 2016). In other words, ASD participants mentally rotate the world towards their own perspective. Both approaches lead to the correct answer, but a MR strategy is more cognitively demanding, resulting in longer reaction times and decreased accuracy. Interestingly, Kessler \& Wang (2012) showed that participants with high levels of autistic traits, but not diagnosed with ASD, showed reduced embodiment effects, compared to participants with low levels of autistic traits. Whilst impaired embodied perspective-taking in ASD has been reported behaviourally (Pearson et al., 2013), no study to date has investigated the neural correlates of perspective-taking in ASD. 


\subsubsection{Hypotheses}

The major aim of Experiment 1 was to elucidate the specific patterns of oscillatory connectivity underlying perspective-taking in the neurotypical brain, using MEG. Participants performed a modified paradigm from Kessler \& Rutherford (2010), which modulates the angle of disparity between self and other perspectives, whilst also separating perspective-taking from perspective-tracking. Based on the literature reviewed, we expected the TPJ and (v)mPFC to form a mentalizing network synchronised via theta oscillations, related to generating the abstract representation of another's perspective, while activation in parietal body-schema areas and sensorimotor cortex would reflect the required embodied transformation to generate this representation via rotation of the egocentric perspective (Kessler \& Rutherford, 2010; Klaus Kessler \& Thomson, 2010; Surtees et al., 2013). In addition, pACC and IPFC might play key roles in top-down executive control of the underlying embodied transformation and in managing the conflict between physical self and transformed self at the representational level.

Experiment 2 aimed to investigate ASD-related individual differences in the neurocognitive mechanisms underlying perspective-taking, using MEG. We collected data from a group of 18 adolescent participants diagnosed with ASD, and 17 age-matched controls, using the same perspective-taking paradigm, and data analysis pipeline as for Experiment 1. During perspective-taking, it was hypothesised that the control group would adopt an embodied egocentric strategy, resulting in increased theta-band power $(3-7 \mathrm{~Hz})$ within the TPJ, motor and executive control regions, replicating Wang et al., (2016). However, for the ASD group it was hypothesised that a mental rotation strategy would be adopted (Conson et al., 2015; Pearson et al., 2016), resulting in longer reaction times, and oscillatory responses located in visual cortex. Additionally, directed functional connectivity analyses between key regions of interest, should show reduced feedback connectivity for the ASD group, consistent with reports of global brain hypoconnectivity in ASD (Hughes, 2007) and reduced top-down modulation of cortical activity (Kessler, Seymour, \& Rippon, 2016; Palmer, Lawson, \& Hohwy, 2017).

\subsection{Materials and Methods}

\subsubsection{Participants}

For Experiment 1, data were collected from 18 participants (4 male, 14 female, mean age $=27.55, S D=5.86$ ). All participants had normal or corrected to normal vision and no history of neurological or psychiatric illness. Two participants had excessive head movement $(>5 \mathrm{~mm})$, and were excluded from data analysis. 
For Experiment 2, data were collected from 18 participants diagnosed with ASD and 17 age-matched typically developing controls, see Table 6.1. MEG data from 4 of these participants (2 ASD, 2 control) were excluded based on RT results (see Section 6.3.3 and Appendix 6). ASD participants had a confirmed clinical diagnosis of ASD or Asperger's syndrome from a paediatric psychiatrist. Participants were excluded if they were taking psychiatric medication or reported epileptic symptoms. Control participants were excluded if a sibling or parent was diagnosed with ASD.

All experimental procedures complied with the Declaration of Helsinki and were approved by the Aston University, Department of Life \& Health Sciences ethics committee. Written consent was obtained from participants aged 18 or over, or a parent/guardian for participants aged under 18.

\begin{tabular}{|c|c|c|c|c|c|c|c|}
\hline & $\underline{\mathbf{N}}$ & Age & Male/Female & $\frac{\frac{\text { Autism }}{\text { Quotient }}}{\underline{\text { Adult) }}}$ & $\frac{\begin{array}{c}\text { Raven } \\
\text { Matrices }\end{array}}{\underline{\text { Score }}}$ & $\begin{array}{c}\text { Glasgow } \\
\underline{\text { Sensory }} \\
\underline{\text { Score }}\end{array}$ & $\begin{array}{l}\text { Mind } \\
\text { in the } \\
\text { Eyes } \\
\text { Score }\end{array}$ \\
\hline ASD & 18 & 16.67 & $\begin{array}{c}14 \text { male; } 4 \\
\text { female }\end{array}$ & $32.6^{*}$ & 43.8 & $65.3^{*}$ & 21.8 \\
\hline Control & 17 & 16.41 & $\begin{array}{c}14 \text { male; } 3 \\
\text { female }\end{array}$ & 10.71 & 48.8 & 38.4 & 25.5 \\
\hline
\end{tabular}

Table 6.1. Participant demographic and behavioural data for Experiment 2. * $=$ behavioural scores significantly greater in ASD>control group, $t$-test, $p<.05$.

\subsubsection{Experimental Paradigm and Design}

The paradigm was adopted from a behavioural study by Kessler and Rutherford (2010). The stimuli were coloured photographs (resolution of $1024 \times 768$ pixels), showing an avatar seated at a round table shown from one of four possible angular disparities. In each trial, one of the grey spheres on the table turned red indicating this sphere as the target. From the avatar's viewpoint, the target could be either visible or occluded (VO) by a centrally resented black screen, Figure $6.2 \mathrm{C} / \mathrm{D}$; or to the left or to the right (LR), Figure $6.2 \mathrm{~A} / \mathrm{B}$, inducing perspective-tracking or perspective-taking, respectively. Stimuli were 
presented in 12 mini-blocks of 32 trials, alternating between LR and VO conditions. On each trial, participants were asked to make a target location judgement according to the avatar's perspective by pressing the instructed key on an MEG-compatible response pad: the left key for "left" or "visible" targets from the avatar's viewpoint and the right key for "right" or "occluded" targets. Accuracy feedback was provided after each trial in the form of a short tone. As in Kessler and Rutherford (2010), we collapsed across clockwise and anticlockwise disparities, and separately collapsed correct responses for left and right and visible and occluded, respectively. This resulted in four separate experimental conditions (for examples see Figure 6.2): left/right judgements where the avatar is $160^{\circ}$ from own perspective (LR-160); left-/right judgements where the avatar is $60^{\circ}$ from own perspective (LR-60); visible/occluded judgments where the avatar is $160^{\circ}$ from own perspective (VO160 ); visible/occluded judgments where the avatar is $60^{\circ}$ from own perspective (VO-60). This $2 \times 2$ design allowed us to disentangle perspective-taking from perspective-tracking and investigate the effect of an increased angle of disparity, which has been shown to lengthen reaction times during perspective-taking (Kessler \& Rutherford, 2010; Surtees et al., 2013). We chose to use $160^{\circ}$ vs. $60^{\circ}$ based on the results of Gooding-Williams et al., (2017) and Wang et al., (2016).

"Is the target to the left or the right of the other person's perspective?"

A

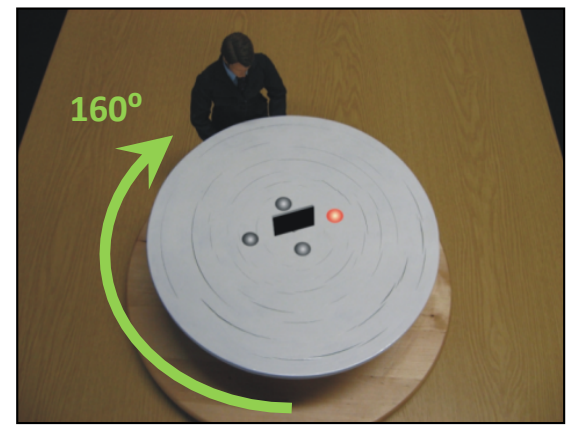

B

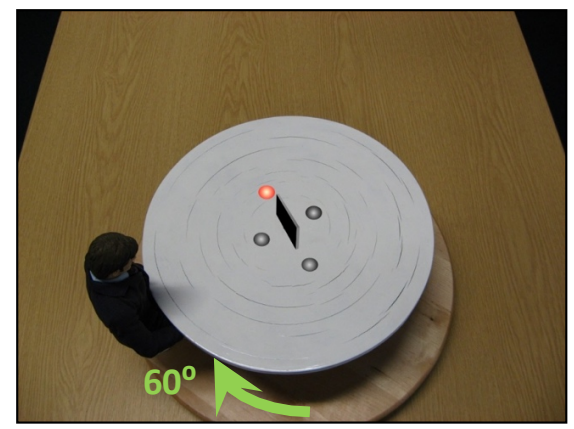

"Is the target visible or hidden from the other person's perspective?"

C

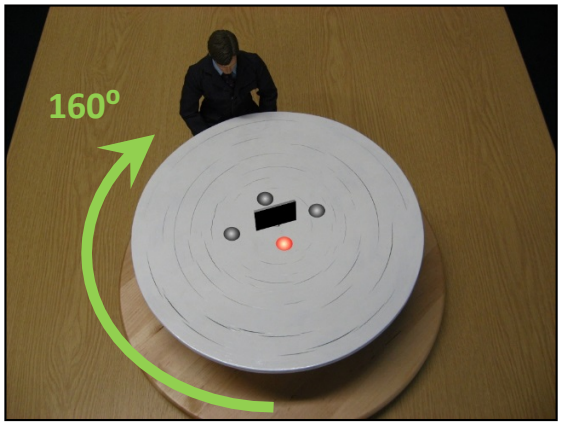

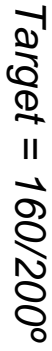

D

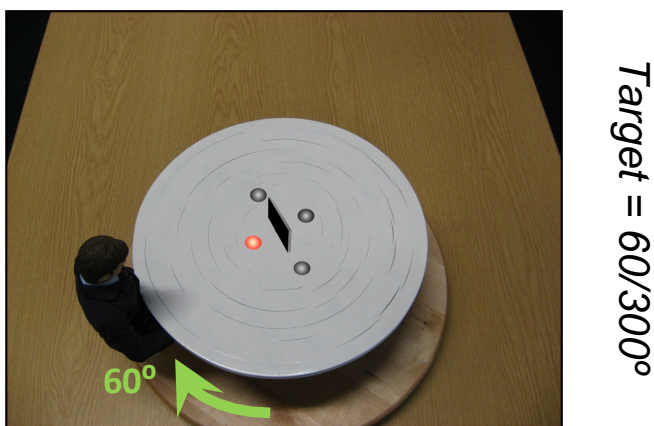


Figure 6.2. Example stimuli from the perspective taking paradigm, for each of the four experimental conditions: (A) LR-160; (B) LR-60; (C) VO-160; (D) VO-60. Arrows are for illustrative purposes and weren't presented to participants.

\subsubsection{Behavioural Data Analysis}

Behavioural reaction times (RT) from the experimental paradigm were extracted from EPrime ${ }^{\circledR}$ data files and converted to .csv format. All trials containing incorrect answers or response times greater than 2 standard deviations from the participant's individual median reaction times (across all experimental conditions) were excluded from subsequent analyses. For the four experimental conditions (LR-160; LR-60; VO-160; VO-60), median RT from each participant were entered into a one-way ANOVA using the JASP statistics package. In Experiment 2, data from 4 participants (2 ASD, 2 control) were discarded due to a median RT greater than 2 standard deviations from the group median (see Appendix $6)$.

\subsubsection{MEG and Structural MRI Acquisition}

MEG data were acquired using a 306-channel Neuromag MEG scanner (Vectorview, Elekta, Finland) made up of 102 triplets of two orthogonal planar gradiometers and one magnetometer. All recordings were performed inside a magnetically shielded room at a sampling rate of $1000 \mathrm{~Hz}$. Five head position indicator (HPI) coils were applied for continuous head position tracking, and visualised post-acquisition using an in-house Matlab script. For MEG-MRI coregistration purposes three fiducial points, the locations of the HPI coils and 300-500 points from the head surface were acquired using the integrated Polhemus Fastrak digitizer. Visual stimuli were presented on a projection screen located $86 \mathrm{~cm}$ from participants, and auditory feedback through MEG-compatible headphones. Data acquisition was broken down into three sequential runs, each lasting 810 minutes.

A structural T1 brain scan was acquired for source reconstruction using a Siemens MAGNETOM Trio 3T scanner with a 32-channel head coil $(T E=2.18 \mathrm{~ms}, T R=2300 \mathrm{~ms}$, $\mathrm{TI}=1100 \mathrm{~ms}$, flip angle $=9^{\circ}, 192$ or 208 slices depending on head size, voxel-size $=$ $0.8 \times 0.8 \times 0.8 \mathrm{~cm})$.

\subsubsection{MEG Preprocessing}

All MEG data were pre-processed using Maxfilter (temporal signal space separation, .9 correlation), which supresses external sources of noise from outside the head (Taulu and Simola 2006). To compensate for head movement between runs, data from runs 2 and 3 
were transformed to participant's head position at the start of the first block using the trans option of Maxfilter. For each participant, the entire recording was band-pass filtered between $0.5-250 \mathrm{~Hz}$ (Butterworth filter) and band-stop filtered to remove residual $50 \mathrm{~Hz}$ power-line contamination and its harmonics. Data were then epoched into segments of $2.5 \mathrm{~s}$ (1s pre, $1.5 \mathrm{~s}$ post stimulus onset) and each trial was demeaned and detrended. Trials containing artefacts (SQUID jumps, eye-blinks, head movement) were removed by visual inspection, resulting in removal of an average of 86.5 trials per condition, per participant. Four MEG channels containing large amounts of non-physiological noise were removed from all source-level analyses. The pre-processed data were then separated into the four experimental conditions and downsampled to $200 \mathrm{~Hz}$ to aid computation time.

\subsubsection{MEG-MRI Coregistration}

MEG data were co-registered with participants' T1 MRI structural scan by matching the digitised head shape data with surface data from the structural scan (Jenkinson \& Smith, 2001). Subsequently, the aligned MRI-MEG image was used to create (1) a forward model based on a single-shell description of the inner surface of the skull (Nolte, 2003), using the segmentation function in SPM8 and (2) spatial normalisation parameters to create individual volumetric grids. To facilitate group analysis, each individual volumetric grid was warped to a template based on the MNI brain, $8 \mathrm{~mm}$ resolution. Subsequently the inverse of the normalisation parameters were applied to the template grid, for source analysis.

\subsubsection{Sensor Level Analysis}

Sensor-level time-frequency representations (TFRs) were calculated using a single Hanning taper between frequencies of $2-30 \mathrm{~Hz}$ in steps of $1 \mathrm{~Hz}$. The entire $2.5 \mathrm{~s}$ epoch was used, with a sliding window of $0.5 \mathrm{~s}$, but the first $0.25 \mathrm{~s}$ and last $0.5 \mathrm{~s}$ of each trial were discarded to avoid edge artefacts. Due to different scales between the two MEG sensortypes, only data from the gradiometers were used, with TFR power averaged across each pair post-hoc. All analyses were computed on single trials and subsequently averaged, and therefore TFRs contain both phase-locked (evoked) and non phase-locked (induced) information.

Based on the results of Wang et al., (2016), for statistical testing, we compared thetaband $(3-7 \mathrm{~Hz})$ and alpha $(8-12 \mathrm{~Hz})$ power during trials in which the avatar was $160^{\circ}$ versus $60^{\circ}$ from the participant's own perspective (clockwise or anticlockwise), in both left/right judgements (i.e. perspective-taking), and visible/occluded judgements (i.e. perspective- 
tracking). We corrected for multiple comparisons across time, frequency and space via cluster-based non-parametric permutation testing (Maris \& Oostenveld, 2007).

\subsubsection{MEG Source-Level}

Source localisation was conducted using Dynamical Imaging of Coherent Sources (DICS; Gross et al. 2001) which applies a spatial filter to the MEG data at every voxel of a canonical $0.8 \mathrm{~cm}$ brain-grid, in order to maximise signal from that location whilst attenuating signals elsewhere. The spatial filter was calculated from the cross-spectral densities for a time-frequency tile centred on the effects found at sensor level, see Results for Experiment 1 and 2. For all analyses, a common filter across baseline and active periods was used (see Appendix 4 and the Fieldtrip tutorial on common filters: https://bit.ly/2t5P9cS). Due to rank reduction following Maxfilter, a regularisation parameter of lambda $5 \%$ was applied to the covariance matrix ( $\mathrm{ft}$ _sourceanalysis option: $\mathrm{cfg} .1 \mathrm{cmv} \cdot 1 \mathrm{ambda}={ }^{\prime} 5 \%$ '). For a graphical illustration of the procedure used for source localisation see Appendix 4.

For statistical testing of the LR-160>LR-60 contrast, cluster-based non-parametric permutation testing was used to correct for multiple comparisons across voxels (Maris \& Oostenveld, 2007). The resulting whole-brain statistical maps were presented on a cortical mesh using the Connectome Workbench software (Van Essen et al., 2012).

Using the common spatial filter computed during source analysis, we extracted trial-bytrial time-courses from regions of interest (see Table 6.2 for more details and specific MNI co-ordinates).

\begin{tabular}{ccc}
\hline Region & MNI Coordinates & Method for Defining \\
\hline $\begin{array}{c}\text { Right temporoparietal Junction } \\
\text { (rTPJ) }\end{array}$ & $40-5836$ & $\begin{array}{c}\text { Maximum t-value within } \\
\text { significant cluster, LR-160>LR- } \\
60 \text { (Experiment 1) }\end{array}$ \\
& & \\
$\begin{array}{c}\text { Right Anterior Cingulate Cortex } \\
\text { (rACC) }\end{array}$ & 123628 & $\begin{array}{c}\text { Maximum t-value within } \\
\text { significant cluster, LR-160>LR- } \\
60 \text { (Experiment 1) }\end{array}$
\end{tabular}


Lateral Pre-frontal Cortex (PFC), Experiment 1

421216

$14-900$

$44-708$
Maximum t-value within significant cluster, LR-160>LR60 (Experiment 1)

Maximum t-value within significant cluster, control>ASD (Experiment 2)

Middle of the Calcarine_R parcel in the AAL Atlas

Maximum t-value within significant cluster (alpha band), control>ASD (Experiment 2)

Table 6.2. Regions of Interest (ROI) used in this chapter with associated MNI coordinates, and how the coordinates were defined.

\subsubsection{Granger Causality Analysis}

For LR-160 trials, the directed functional connectivity between ROIs was estimated using spectrally-resolved non-parametric Granger causality (Dhamala, Rangarajan, \& Ding, 2008) as implemented in the Fieldtrip toolbox (Oostenveld, Fries, Maris, \& Schoffelen, 2010). Intact and scrambled time-series were split into 0.3s epochs to enhance the accuracy of the results (LR-160 trials, 0.0-0.6 post stimulus onset), followed by Fourier transformation (Hanning taper; $2 \mathrm{~Hz}$ spectral smoothing; 4s zero-padding), before being entered into a non-parametric spectral matrix factorisation procedure. Granger causality was then estimated between each ROI pair and each ROI-scrambled time-series. Statistical analysis was performed using cluster-based permutation testing, to compare real GC values with surrogate GC values. $p$-values were Bonferroni-corrected across ROIpairs (.05/6 ROI pairs for Experiment 1; .05/12 ROI pairs for Experiment 2).

\subsubsection{Statistical Analysis}

For MEG data, statistical analysis was performed using cluster-based permutation tests (Maris \& Oostenveld, 2007), which consist of two parts: first an independent-samples ttest is performed, and values exceeding an uncorrected $5 \%$ significance threshold are grouped into clusters. The maximum t-value within each cluster is carried forward. Second, a null distribution is obtained by randomising the condition label (e.g. 
ASD/control) 1000 times and calculating the largest cluster-level t-value for each permutation. The maximum t-value within each original cluster is then compared against this null distribution, and the null hypothesis is rejected if the test statistic exceeds a threshold of $p<.05$. For tables outlining significant clusters and $\mathrm{MNI}$ co-ordinates from whole-brain statistical maps, please refer to Appendix 5.

\subsection{Results - Experiment 1}

Please note, these results have been published in a peer-reviewed journal (Seymour, Wang, Rippon, \& Kessler, 2018).

\subsubsection{Behavioural Results}

For the four experimental conditions, median reaction times (RT) from each participant were entered into a one-way ANOVA. Results showed a main effect of experimental condition on $\mathrm{RT}, \mathrm{F}(3,60)=4.43, \mathrm{p}=.007, \eta^{2}=0.181$. Post-hoc tests revealed this was due to significantly longer RT for the LR-160 conditions compared with all other conditions $\left(\right.$ LR-60, $\left.p_{\text {tukey }}=.013 ; \mathrm{VO}-160, \mathrm{p}_{\text {tukey }}=.029 ; \mathrm{VO}-60, \mathrm{p}_{\text {tukey }}=.026\right)$, replicating Kessler and Rutherford (2010).

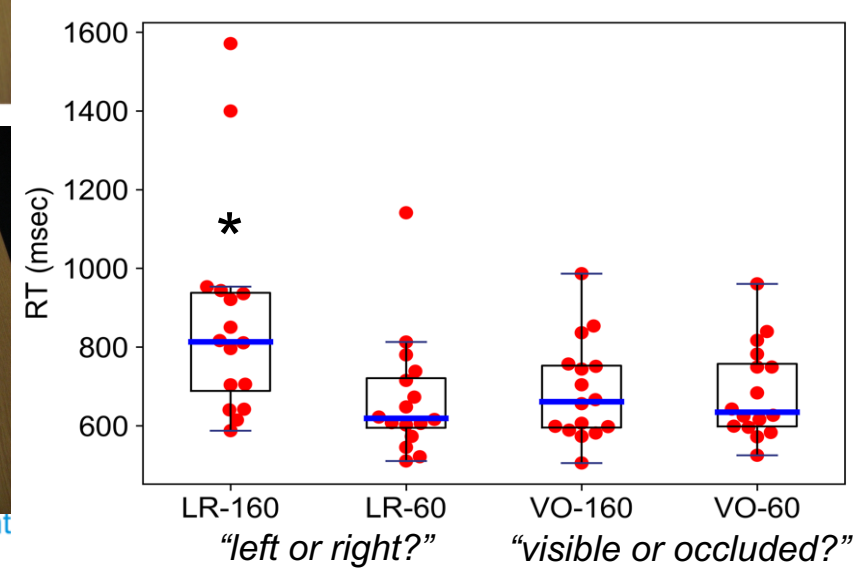

Figure 6.3 Behavioural results, Experiment 1. Boxplots showing reaction time (RT) data were produced for each of the four experimental conditions. Red dots correspond to individual participant median RTs. ${ }^{*}=L R-160$ is significantly different from all other conditions $(p<.05)$.

\subsubsection{Task-Related Changes in Theta Power}

Using a data-driven approach from $2-30 \mathrm{~Hz}$, time-frequency results at the sensor-level, Figure 6.4, replicated the crucial role of theta oscillations in perspective-taking (GoodingWilliams et al., 2017; Wang et al., 2016). A significant positive cluster $(p=0.03)$ was found at $3-6 \mathrm{~Hz}, 0-0.65 \mathrm{~s}$, when comparing angular disparities of $160^{\circ}$ and $60^{\circ}$ degrees for $L / R$ 
perspective-taking trials (Figure 6.4 left panel). No significant effects were found for any other frequencies ( $p>.05$ ). In addition, no significant clusters were found for the V/O condition, i.e. when comparing angular disparities of $160^{\circ}$ and $60^{\circ}$ degrees for $\mathrm{V} / \mathrm{O}$ perspective-tracking trials (Figure 6.4 right panel).

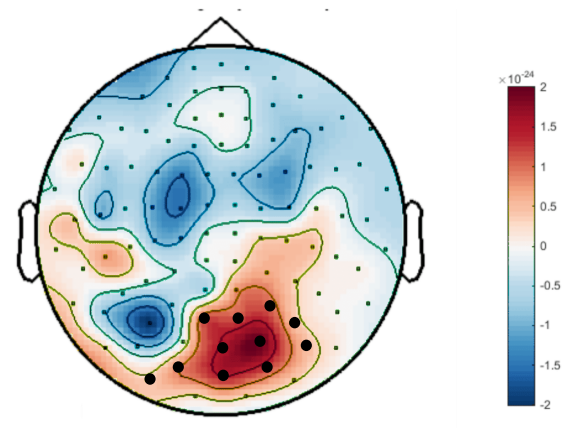

LR-160 > LR-60

$$
\begin{gathered}
\text { 3-6 } \mathrm{Hz} ; 0-650 \mathrm{~ms} \\
\text { post-stimulus onset } \\
\begin{array}{c}
\text { Perspective-Taking } \\
\text { (Level 2) }
\end{array}
\end{gathered}
$$

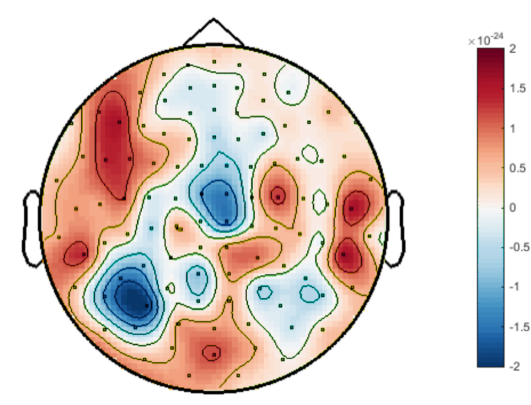

VO-160 > VO-60

$$
3-6 \mathrm{~Hz} ; 0-650 \mathrm{~ms}
$$

post-stimulus onset

\section{Perspective-Tracking (Level 1)}

Figure 6.4. Topoplots of oscillatory power between 3-6Hz, 0-0.65s post-stimulus onset, for LR-160 > LR-60 (left panel) and VO-160 > VO-60 (right) contrasts. Sensors showing a significant, $p<.05$, difference between $L R-160$ and LR-60 trials are highlighted with a boldened black dot. Scales represent MEG field strength, baseline-corrected, with units of Tesla/ $\mathrm{cm}^{2}$.

Based on these sensor-level data, we decided to concentrate on further characterising theta-band power and connectivity underlying perspective-taking. Whilst a wider frequency range, that also included higher delta frequencies, has been used to define theta-band power in previous studies (Wang et al., 2016), we opted to use 3-6Hz, based on the statistical analysis of the sensor-level data in order to achieve the best signal-tonoise ratio for subsequent beamforming analyses.

To investigate the cortical sources underlying this effect of angular disparity, theta-band $(3-6 \mathrm{~Hz})$ power was localised from $0-0.65 \mathrm{~s}$ post-stimulus onset separately for $160^{\circ}$ and $60^{\circ}$ trials, using the Dynamic Imaging of Coherent Sources (DICS) approach, see Section 6.3.8 Materials and Methods. Baseline-corrected theta $(3-6 \mathrm{~Hz})$ power was compared between LR-160 and LR-60 trials, across a $0.8 \mathrm{~cm}$ cortical grid. Results showed a 
significant, $p<.05$, increase in theta power during LR-160 trials compared with LR-60 trials for right posterior temporo-parietal junction (pTPJ) spreading into the inter-parietal sulcus (IPS), for right lateral pre-frontal cortex (PFC) primarily overlapping with the inferior frontal gyrus (IFG) and for right anterior cingulate cortex (ACC), see Figure 6.5A. There was also a decrease in theta power in the LR-160 versus LR-60 condition in the left frontal pole (see Appendix 5).
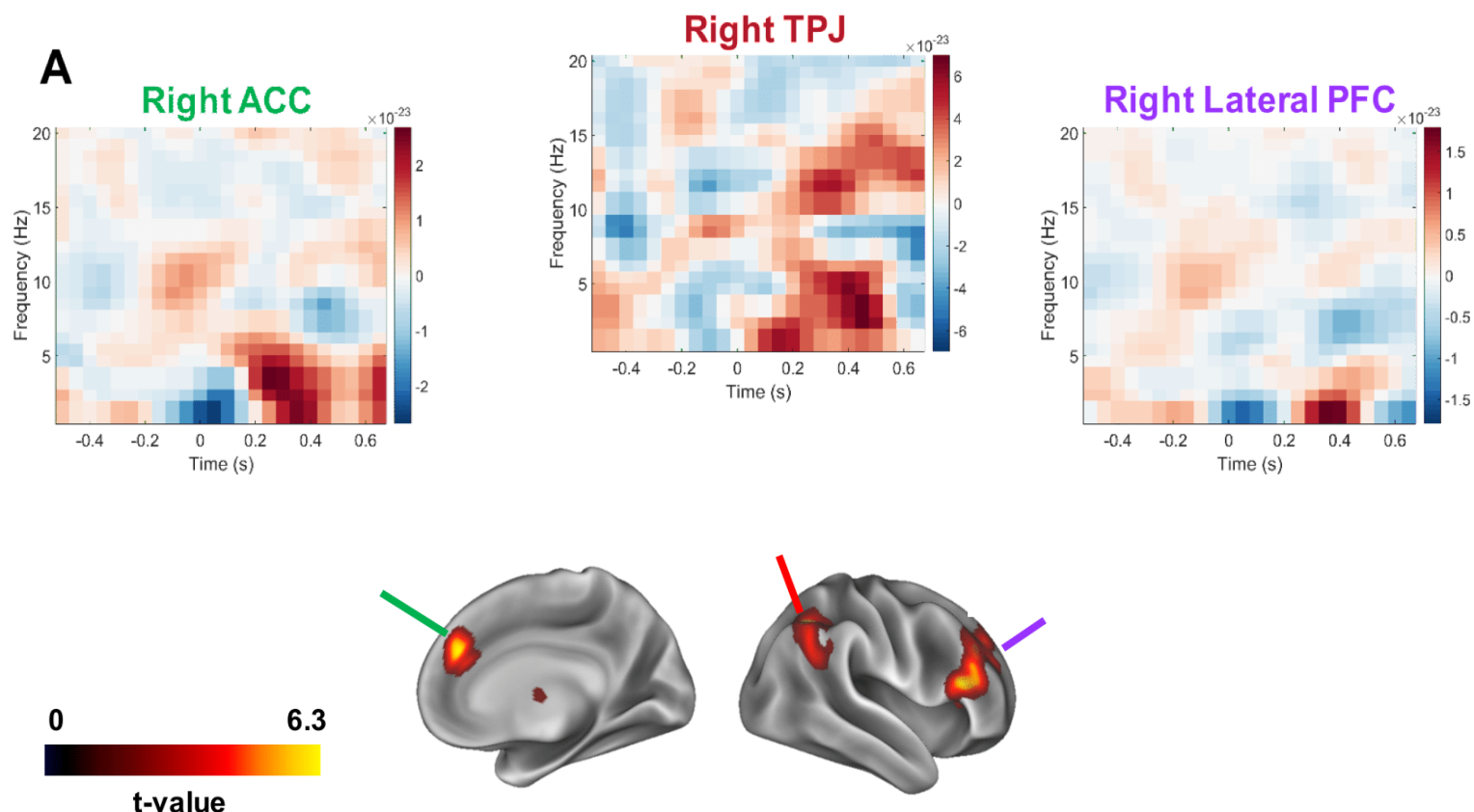

Right Hemisphere
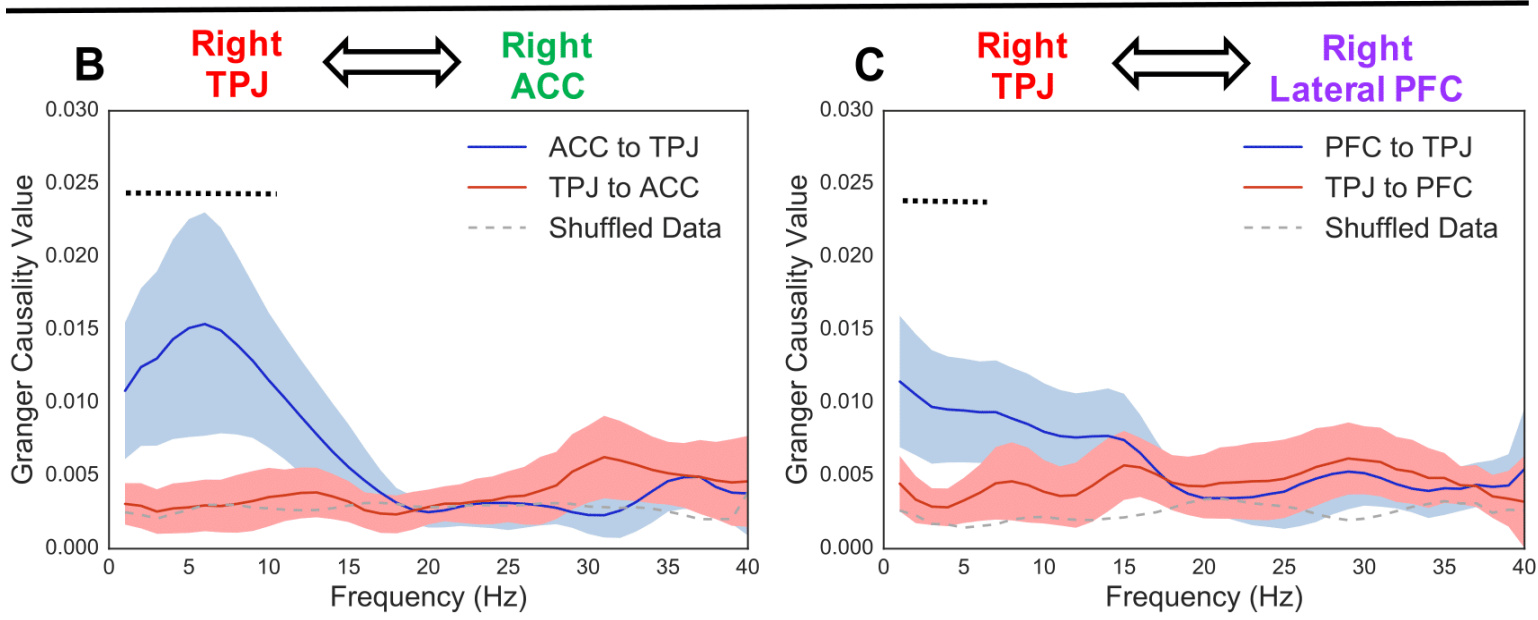

Figure 6.5: Theta power sources and directed connectivity. Panel A shows brain plots showing statistical results (clusters with $p<.05$ are shown) of a whole-brain DICS theta power (3-6Hz) analysis for $L R-160>L R-60$ contrast visualised using the Connectome Workbench software (Van Essen et al., 2012). Plots at the top show time-frequency representations ( $L R-160>L R-60$ contrast) for three regions of interest. Panel B shows spectrally resolved non-parametric Granger causality $(1-40 \mathrm{~Hz})$, computed between the right TPJ-ACC and rTPJ- right lateral PFC. Results show an increase in Granger causality 
from both the right ACC $(1-10 \mathrm{~Hz})$ and right PFC $(1-5 \mathrm{~Hz})$ to the right TPJ. Shaded regions around each line represent $95 \%$ confidence intervals. The black dotted line above the plots represents Granger causality values passing a $p<.05$ threshold, when statistically comparing each direction of an ROI-pair (i.e. red and blue lines, see Section 6.3.9-10 for details). The grey dotted line in the plots shows shuffled data for comparison. Further explanations in the text.

\subsubsection{Virtual Electrode Time-Frequency Analysis}

To further investigate the oscillatory signatures of high-level perspective-taking, timecourses for each trial were extracted from 'virtual-electrodes' identified above in right TPJ, right ACC and right lateral PFC, see Section 6.3 Materials \& Methods, Table 6.2 and Appendix 4 for details. Low-frequency oscillatory power was then estimated between 0.65 to 0.65 s post-stimulus using a Hanning taper, 0.05 s sliding window. Results show very early and sustained theta power $(3-6 \mathrm{~Hz})$ increases in the right TPJ $(0-0.5 \mathrm{~s})$ for LR160 versus LR-60 trials. Right lateral PFC delta/theta power $(1-5 \mathrm{~Hz})$ and right ACC (1$5 \mathrm{~Hz}$ ) increases are more transient and begin from $0.2-0.5$ s post-stimulus onset. This suggests that the rTPJ is engaged throughout the process of embodied perspectivetaking, whereas increases in theta power ACC and PFC occur later and more transiently.

\subsubsection{Granger Causality Analysis}

To investigate directed functional connectivity during perspective-taking between the three main regions of interest, ROIs, identified in the source power analysis (rTPJ, rACC and rPFC, also see Table 6.2), we employed spectrally resolved non-parametric Granger causality (GC) on LR trials, $0-0.65$ s post-stimulus onset (Dhamala et al., 2008). GC is a statistical concept used to estimate directed connectivity between time-series, which relies on the premise that if the time-series of region $A$ can be used to predicts the time-series of region $B$, then $A$ is said to 'granger-cause' $B$ (Ding Mingzhou et al. 2006). GC can also be extended to the frequency domain, as discussed in Bastos \& Schoffelen (2016). Spectrally-resolved GC therefore provides information about the direction of connectivity between regions of interest, as well as the frequency-band underlying the effects.

Between the three ROls, GC values showed statistically significant, $p<.05 / 6$, difference from fourier-scrambled time-series in two pairs: rTPJ-rACC and rTPJ-rPFC. To investigate these effects further, we statistically compared GC values between each direction of the ROI pair (i.e. the granger causal influence to and from the rTPJ). Results showed an asymmetric increase in granger causal influence, directed from right $\mathrm{ACC}$ between 1- 
$10 \mathrm{~Hz}$, with a peak at $6 \mathrm{~Hz},($ Fig. 6.5B, $\mathrm{p}=.009)$ and right PFC, between 1-6Hz, (Fig 6.5C, $p=.04)$ to the right TPJ.

\subsubsection{Imaginary Coherence}

Phase-synchronised neural activity has been argued to act as a mechanism for information flow between brain regions during cognitive tasks (Womelsdorf et al., 2007). Measures of phase synchrony (e.g. coherence) can therefore provide information about changes in brain connectivity within a particular frequency band. However unlike GC, coherency alone does not provide information about the direction of connectivity.

To establish patterns of whole-brain functional connectivity accompanying righthemisphere TPJ theta-band activity, we extracted source-level theta-band $(5 \pm 2 \mathrm{~Hz})$ phase relationships from the sensor-level cross-spectral density matrix (see Section 6.3 Materials and Methods). A measure of phase synchrony between a right-TPJ seed and every other voxel was calculated by projecting complex-valued coherency onto the imaginary axis (Nolte et al., 2004). The resulting coherency maps from the LR-160 and LR-60 conditions were first baseline-corrected, and then compared using cluster-based non-parametric permutation testing (Maris \& Oostenveld, 2007).

Results, see Figure 6.6, show a complex pattern of both increased and decreased thetaband phase synchrony during embodied perspective-taking. The main areas of decreased synchrony are located in the ventral occipitotemporal cortex (VOTC), overlapping with key regions of the ventral visual stream. There were also reductions in phase synchrony to the bilateral anterior temporal lobes (ATL). Increased phase synchrony was observed in bilateral medial PFC regions, posterior cingulate cortex (PCC), intra-parietal sulcus (IPS), supplementary motor area (SMA), posterior parietal cortex (PPC), and right supramarginal gyrus/sensorimotor cortex (SMC). These patterns of phase synchrony are unlikely to be driven by spurious connectivity from MEG field spread (Brookes et al., 2011), as we opted to measure imaginary coherence (Nolte et al., 2004), thereby removing effects in relation to instantaneous phase. 


\section{Left Hemisphere Right Hemisphere}

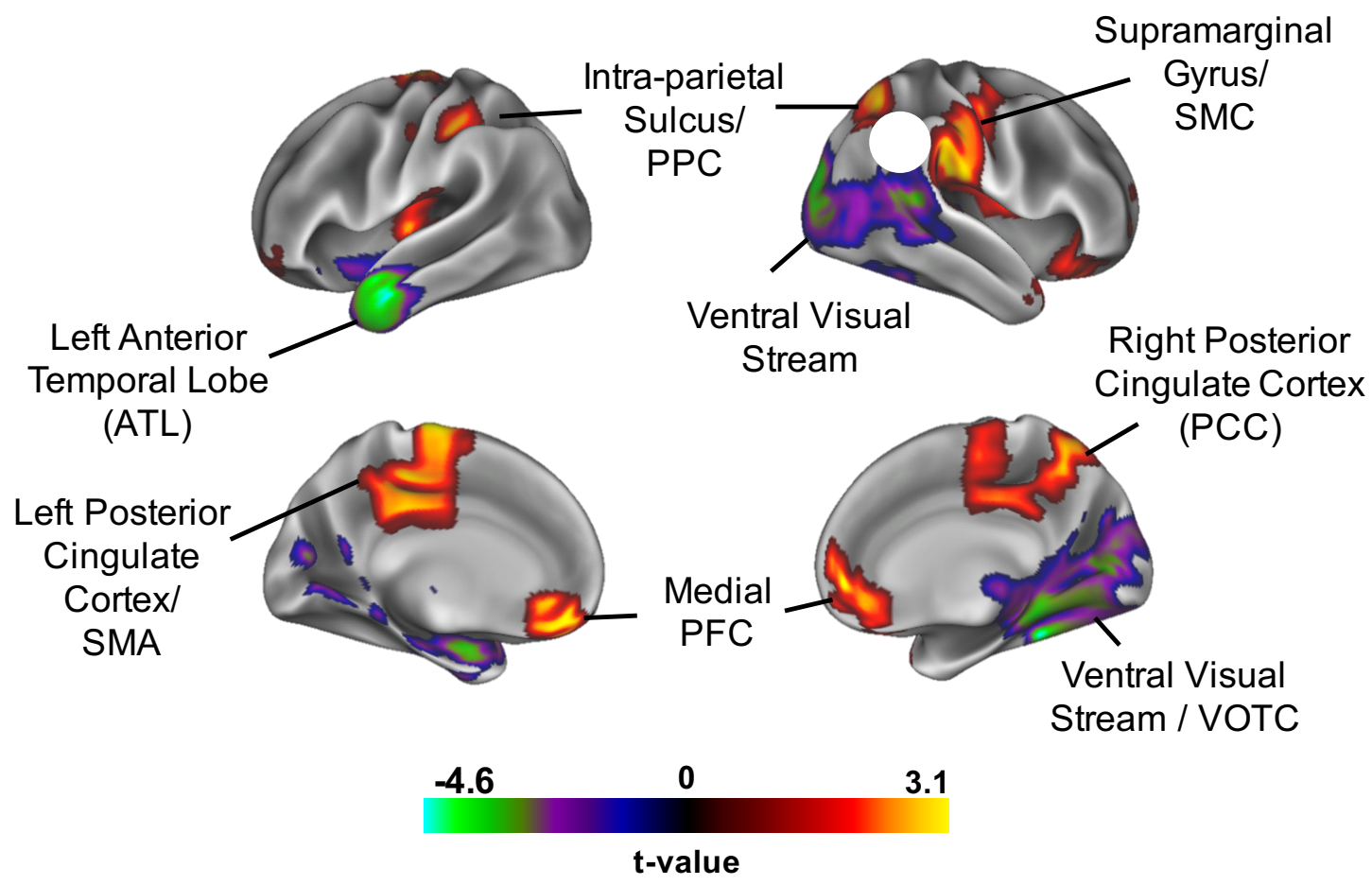

Figure 6.6: Results of a whole-brain imaginary coherence analysis in relation to a right TPJ seed (white circle) and for a LR-160 > LR-60 contrast, visualised using the Connectome Workbench software (Van Essen et al., 2012). Clusters of coherency increase/decrease passing a $p<.05$ threshold are shown, see Section 6.3 Material and Methods. $P P C=$ posterior parietal cortex; $S M C=$ sensorimotor cortex; SMA = supplementary motor area; PFC = prefrontal cortex; VOTC = ventral occipitotemporal cortex.

\subsection{Results - Experiment 2}

\subsubsection{Behavioural Results}

Median reaction times (RT) from each participant were entered into an ANOVA, with condition (LR-160, LR-60, VO-160, VO-60) and group (ASD, control) as factors. Results showed a main effect of experimental condition on $\mathrm{RT}, \mathrm{F}(3,116)=8.715, \mathrm{p}<.001, \eta^{2}=$ 0.170, see Figure 6.7. Post-hoc tests revealed this was due to significantly longer RT for the LR-160 conditions compared with all other conditions ( $p_{\text {tukey }}<.001$ ), replicating Experiment 1, Wang et al., (2016), and Kessler and Rutherford (2010). There was also a main effect of group on $R T, F(1,116)=9.400, p=.003, \eta^{2}=0.061$. A simple main effects analysis showed that this was driven by longer RT for ASD participants, specifically in the LR-160 condition ( $p=.005$, all other conditions were $p>.05$ ). 
As Kessler \& Wang (2012) showed that embodiment was related to Autism Quotient (AQ) scores, we performed a correlation between the angle of disparity (RT difference between LR-160 vs LR-60 trials) and AQ score across both groups. However, this correlation was not significant, Pearson's $r=0.057, p=.762$.

A

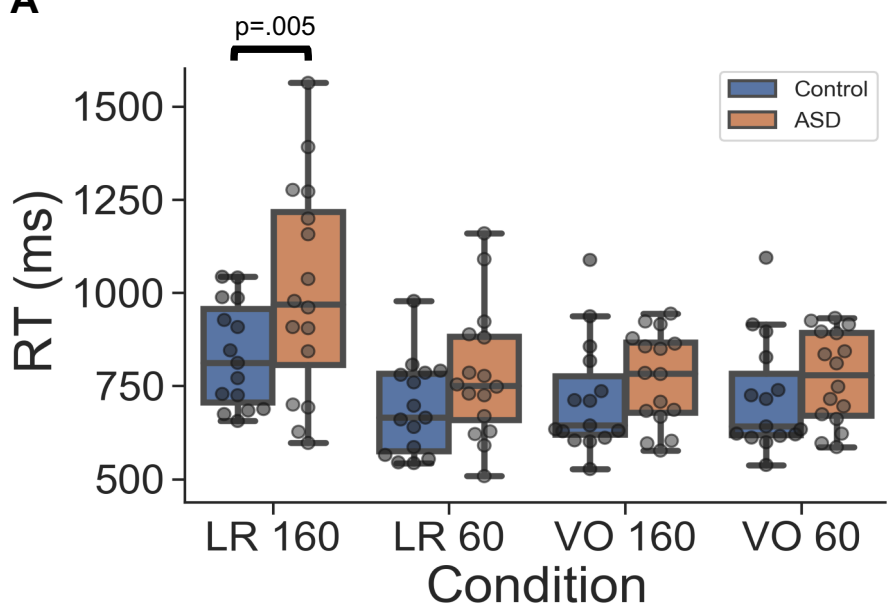

B

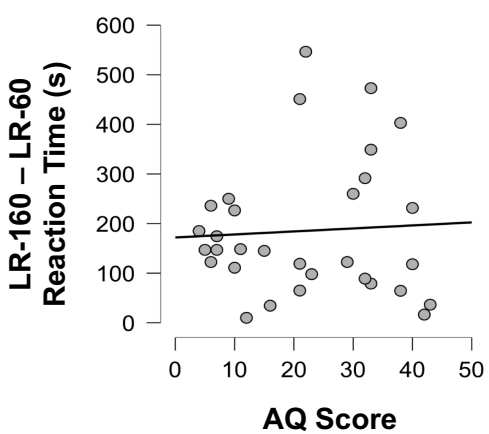

Figure 6.7 Behavioural results, Experiment 2. (A) Boxplots showing reaction time (RT) data are shown for each of the four experimental conditions. Grey dots indicate individual participant RT medians per condition. Significant differences between groups are shown. (B) Scatter plot with regression line to show the relationship between angle of disparity and $A Q$ score across both $A S D$ and control groups.

\subsubsection{Task-Related Changes in Oscillatory Power}

For the control group at the sensor-level, statistically comparing LR-160 versus LR-60 trials $(2-30 \mathrm{~Hz})$, there was one significant cluster of greater $(3-6 \mathrm{~Hz})$ theta-band power at 0 $0.6 \mathrm{~s}$ in the LR-160 versus LR-60 condition, $p=.037$, Figure $6.8 \mathrm{~A}$ left subpanel. For the same comparison in the ASD group, there was a significant cluster of decreased alphaband power $(8-12 \mathrm{~Hz})$ at $0.2-0.85 \mathrm{~s}, \mathrm{p}=.019$, Figure $6.8 \mathrm{~A}$ right subpanel. For both groups there were no significant differences in time-frequency power between VO-160 and VO-60 trials (i.e. perspective tracking), suggesting that theta and alpha effects are specific for embodied perspective-taking (level 2) rather than perspective-tracking (level 1), Figure 6.8B. 


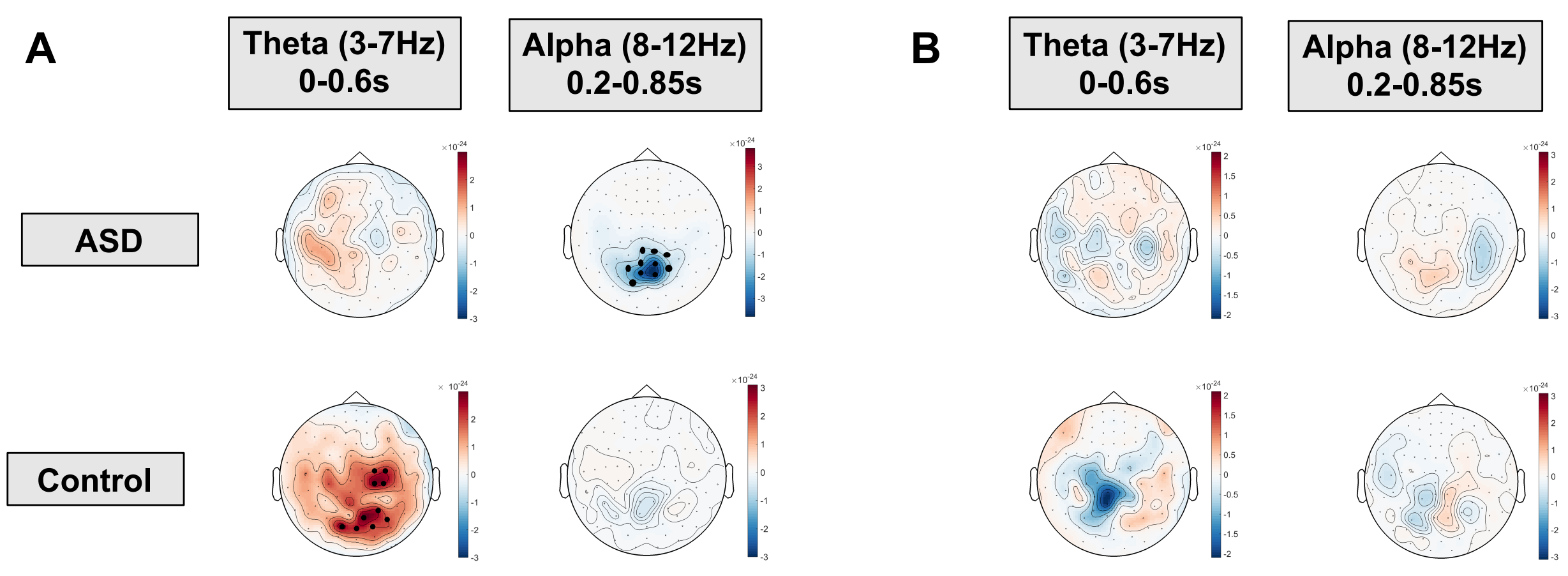

Figure 6.8 Sensor-Level topoplots (gradiometers only) are shown for the difference in oscillatory power between (A) LR-160 > LR-60 trials (i.e. perspective-taking) and (B) VO-160 > VO-60 trials (i.e. perspective-tracking). Theta (3-7Hz) and alpha (8-12Hz) power are plotted separately for 0$0.6 s$ and 0.2-0.85 respectively. Sensors showing a significant difference, $p<.05$, between conditions are highlighted with a boldened black dot. Scales represent MEG field strength, baseline-corrected, with units of Tes/a/ $\mathrm{cm}^{2}$. 
To investigate the cortical sources underlying this effect of angular disparity for L/R trials, theta-band $(3-6 \mathrm{~Hz})$ power was localised from $0-0.6$ s post-stimulus onset, and alpha power $(8-12 \mathrm{~Hz})$ from 0.2-0.85s, using a Dynamic Imaging of Coherent Sources (DICS) approach, see Section 6.3.8 in Materials and Methods. Statistically comparing power between LR160 versus LR-60 trials in source-space, there were significant, $p<.05$, increases in power for controls, over ventral visual cortex, right lateral pre-frontal cortex (PFC) and the left anterior temporal lobe (Figure 6.9, left panel). There were also increases in theta-band power over the right TPJ, but these failed to reach a significance level of .05 , corrected for multiple comparisons (Maris \& Oostenveld, 2007). The ASD group showed no significant increases in theta-power, Figure 6.9 middle panel. When comparing LR-160 trials versus LR-60 trials between groups (i.e. the difference of differences), controls showed significantly increased theta-power over right ventral occipital cortex, right inferior temporal cortex, and lateral PFC, see Figure 6.9 right panel.
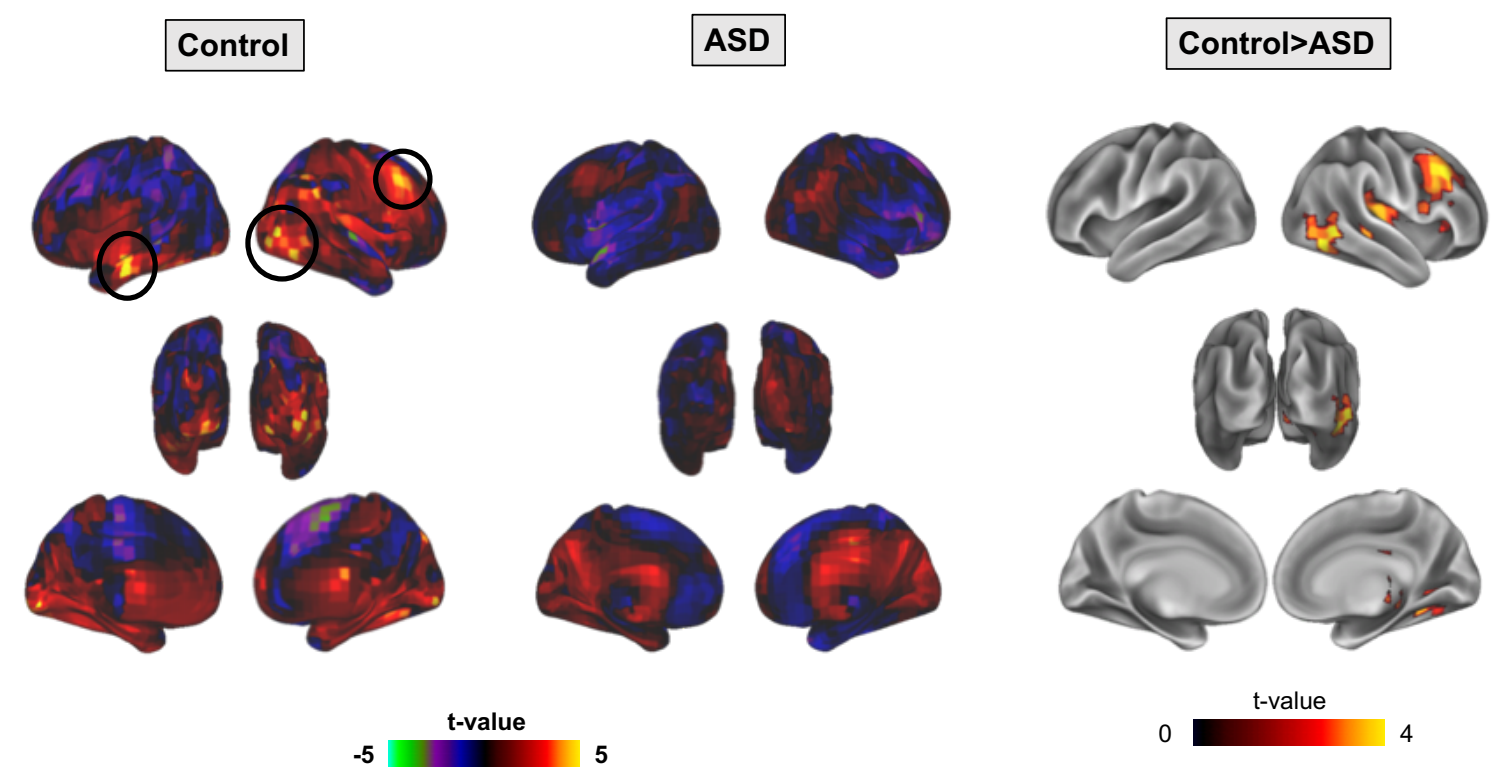

Figure 6.9 Source Localisation of theta-band effects between $L R-160$ and $L R-60$ conditions. (A-B) ASD and Control data is plotted separately, with black circles indicating significant, $p<.05$, positive clusters. (C) For the statistical comparison of Control $>A S D$ groups, whole-brain maps were thresholded at $p<.05$.

Comparing LR-160 versus LR-60 trials in source-space, for the alpha-band, there was a significant, $p<.05$, decrease in power for the ASD group, i.e. alpha desynchronisation, over bilateral occipital cortex, extending into ventral visual areas, Figure 6.10 middle panel. One cluster of activity (peak = [44 -70 8]) overlapped with right V5 - a region implicated in motion processing and object rotation. The control group showed no significant changes in alpha-power, Figure 6.10 left panel. Between groups, there was 
significantly reduced alpha power, i.e. greater alpha desynchronisation, in the ASD compared with control group, overlapping with bilateral visual cortex, Figure 6.10, right panel.

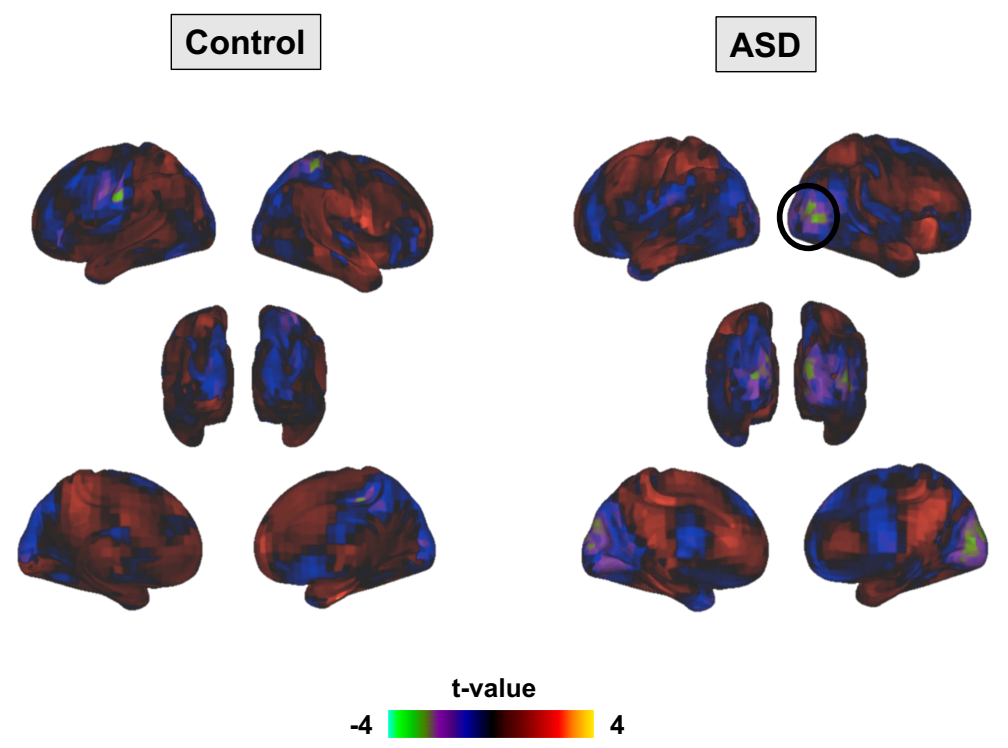

Figure 6.10 Source Localisation of alpha-band effects between LR-160 and LR-60 conditions. (A-B) ASD and Control data is plotted separately, with black circles indicating the approximate location of significant, $p<.05$, positive clusters. (C) For the statistical comparison of Control and ASD groups, whole-brain maps were thresholded at $p<.05$.

Based on these results and our previous results, we defined four regions of interest (ROIs): V1, right V5, right TPJ and right lateral PFC (also see Table 6.2 for more information). V1 showed decreased alpha power for the ASD group, and was included as a ROI in order to investigate how early visual cortex interacts with higher-order regions. Changes in power over ventral visual regions, overlapping with $\mathrm{V} 5$, were reported for both the alpha-band (ASD) and theta-band (Control). Significant changes in rTPJ power were not observed in this experiment after correction for multiple comparisons, but was included as a ROI based on the results of Experiment 1, and Wang et al., (2016). Finally, the right lateral PFC showed differences in theta-power between groups and was also reported for Experiment 1.

\subsubsection{Virtual Electrode Time-Frequency Analysis}

For each ROI, oscillatory power was estimated between -0.65 to 1.0 s post-stimulus, 2$20 \mathrm{~Hz}$ using a Hanning taper, 0.05 s sliding window. Oscillatory power between LR-160 trials was compared with LR-60 trials. For the control group, results show early $(0-0.4 \mathrm{~s})$ increases in theta power for V1 and V5. The lateral PFC shows more sustained patterns 
of theta power (0-0.6s) from $2-5 \mathrm{~Hz}$. The ASD group shows sustained alpha desynchronisation in V1 and V5 from 0.2-1.0s, but not for rTPJ or PFC.
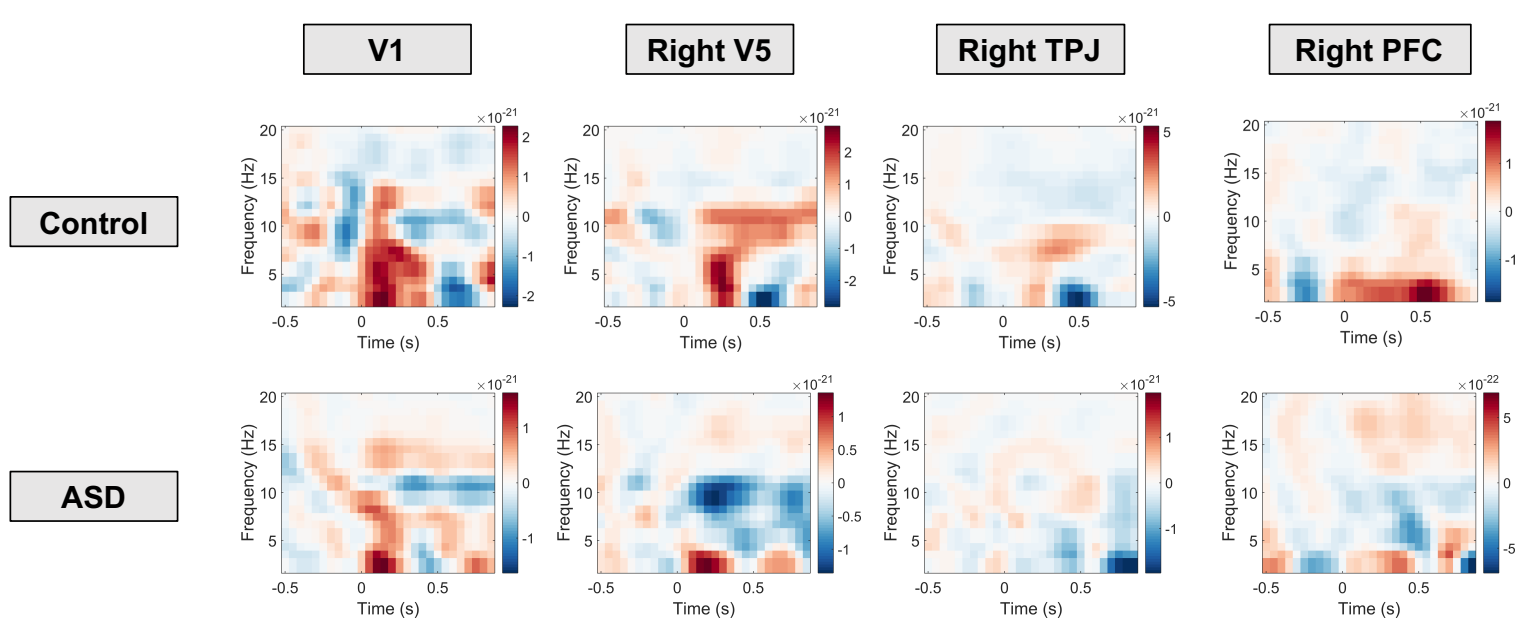

Figure 6.11 Time-Frequency Representations were computed from 2-20Hz, -0.5 to $1.0 \mathrm{~s}$, and compared between $L R-160$ and $L R-60$ trials for each of the $4 R O / s$. The control group (top row) and the ASD group (bottom row) are plotted separately. Scales represent MEG field strength, baseline-corrected, with units of Tesla/ $\mathrm{cm}^{2}$.

\subsubsection{Granger Causality Analysis}

To investigate directed functional connectivity for LR-160 trials, between the four main regions of interest (ROls) identified in the source power analysis (V1, V5, rTPJ, lateral PFC), we employed spectrally resolved non-parametric Granger causality (GC), from 00.65 s post-stimulus onset (Dhamala et al., 2008). For controls, GC values showed statistically significant, $p<.05$ Bonferroni corrected, differences from surrogate data in four ROI-pairs: V1-to-V5, V5-to-V1, rTPJ-to-V5, lateral PFC-to-V5, Figure 6.12 top row. For the ASD group, there were only differences between two ROI-pairs: V1-to-V5, V5-to-V1, Figure 6.12 bottom row. All other ROI-pairs failed to show significant differences, $p<.05$, compared with surrogate data. 


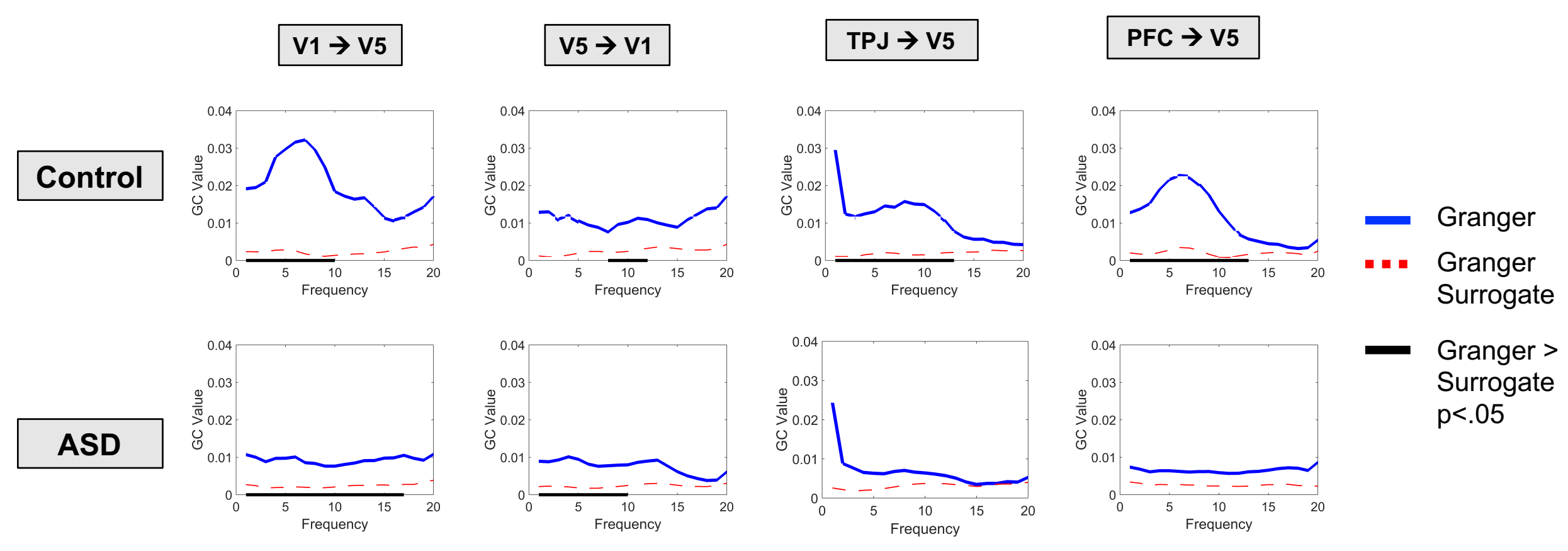

Figure 6.12 Granger Causality (GC) results for selected ROI-pairs, in the control group (top row) and ASD group (bottom row). GC values are plotted in blue, surrogate data is plotted in red. Frequencies showing a significant difference, $p<.05$, between real and surrogate data are indicated by a boldened black line on the $x$-axis. 
Focussing on the four ROI-pairs identified for controls, we compared GC values between ASD and control groups. Results showed that there were significant, $p<.05$, differences between two ROI-pairs: greater GC values for the control group between V1-to-V5 from 5$9 \mathrm{~Hz}$ (theta-band); and greater GC values for the control group between lateral PFC-to-V5 from $4-10 \mathrm{~Hz}$.

A
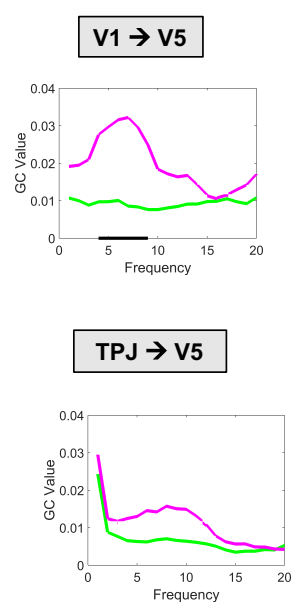

V5 $\rightarrow$ V1

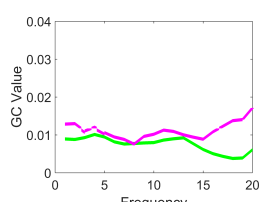

ASD

Control

PFC $\rightarrow$ V5

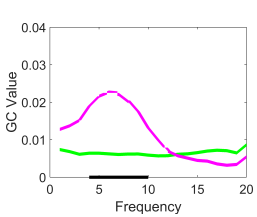

B

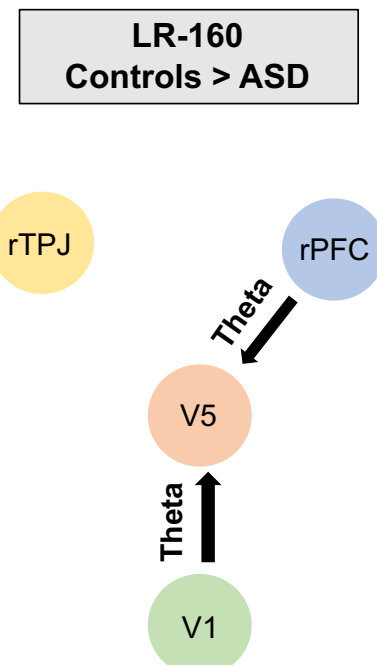

Figure $6.13(\boldsymbol{A})$. Group comparison of Granger Causality (GC) results. ASD data are plotted in green, control data are plotted in purple. Frequencies showing a significant difference, $p<.05$, between Control and ASD groups are indicated by a boldened black line on the $x$-axis. (B) Diagrammatic representation of the GC results. Group differences between the four ROIs are shown with a black line and the associated frequency band of the effect.

\subsection{Discussion}

\subsubsection{Neural Signatures of Perspective-Taking}

In Experiment 1, we investigated the neural signatures of embodied perspective-taking using MEG. Greater angular disparity for perspective-taking was accompanied by greater reaction times, as well as increased theta power $(3-6 \mathrm{~Hz})$ within the right TPJ/IPS and lateral PFC, as well as within the right ACC. Importantly, this increase in theta-power for angular disparity was specific to perspective-taking and not perspective-tracking, see Figure 6.4. We focused on network connectivity between these regions of interest, and showed that there was an increase in Granger causal influence (Dhamala et al., 2008) from lateral PFC and right ACC to right TPJ, but not vice-versa, mediated by low frequency brain rhythms $(1-10 \mathrm{~Hz})$. Finally, whole-brain patterns of theta-band $(5 \pm 2 \mathrm{~Hz})$ phase synchrony were quantified using imaginary coherence (Nolte et al., 2004), in relation to a right TPJ seed region. Results suggest that with increasing angular disparity 
$\left(160^{\circ}\right.$ versus $\left.60^{\circ}\right)$, the right TPJ increases its phase coupling to regions involved in theory of mind (Carrington \& Bailey, 2009) (medial PFC, PCC) and body schema (Coslett et al., 2008; Medina et al., 2009) (SMC, PPC, SMA), but decreases its phase coupling to visual regions (VOTC) and to bilateral anterior temporal lobe (ATL).

Results from Experiment 1 suggest that the right TPJ (rTPJ) becomes increasingly engaged with the need for embodied mental alignment during perspective-taking i.e. as the disparity grows between our own and other's perspectives (Gooding-Williams et al., 2017; Wang et al., 2016). This is consistent with the TPJ's role in establishing a sense of self (Blanke et al., 2005), and crucially in differentiating conflicts between the self and other (Eddy, 2016; Santiesteban, Banissy, Catmur, \& Bird, 2012; Sowden \& Catmur, 2015). As previous research has implicated the TPJ as a major network hub (Igelström and Graziano 2017.; Bzdok et al. 2013), we hypothesised that the region would coordinate shifts in functional connectivity to other brain regions, via phase synchrony (Engel, Fries, \& Singer, 2001; Varela, Lachaux, Rodriguez, \& Martinerie, 2001). Indeed, we found that the rTPJ increased its phase-coupling to the medial PFC and posterior PCC - two regions also involved more generally in understanding the mental states of others (Carrington \& Bailey, 2009; Lieberman, 2007; Van Overwalle, 2009) (i.e. mentalizing). Importantly, phase-coupling at theta frequency between rTPJ and medial PFC had been previously reported by Bögels et al (2015) during a high-level mentalizing task. Thus, TPJmPFC coupling could be part of a basic mechanism involved in simpler as well as in more sophisticated forms of social mental alignment.

Increased phase synchrony was also found between the rTPJ and SMC, SMA, and PPC (see Figure 6.5), which are regions previously implicated in coding for the body schema, i.e. cortical correlates of the internal representation of the body and its postures and actions (Coslett et al., 2008; Medina et al., 2009). This functional link may underlie the simulated rotation of the embodied self into another's orientation and perspective (Arzy, Thut, Mohr, Michel, \& Blanke, 2006; Cazzato, Mian, Serino, Mele, \& Urgesi, 2015). The rTPJ also showed decreased phase synchrony with visual regions (VOTC), primarily the ventral stream of the right visual cortex, during high-level perspective-taking. Altogether, these findings can be interpreted as an active shift from externally-driven processing (i.e. bottom-up sensory information) to internal representations (i.e. self, body-schema rotation). This switch from processing external events to updating internal states and information has been previously linked with TPJ function (Bzdok et al., 2013; Wu et al., 2015). We therefore propose that the rTPJ acts as a hub for high-level perspective taking by routing visual information to internal representations of the self, the body and its action 
and posture repertoire, via theta-band phase synchronisation, see Figure 6.14. This allows humans to actively project their own sense of self into another's viewpoint, resulting in rapid and accurate perspective-taking responses (Gooding-Williams et al., 2017; Kessler \& Rutherford, 2010; Surtees et al., 2013; Wang et al., 2016).

Along with the rTPJ, two additional regions showed significantly increased theta power with increasing angular disparity during perspective-taking: the lateral PFC, primarily overlapping with the right inferior frontal gyrus; and the right ACC (see Figure 6.5). This theta-band activity was found during a slightly later period than the rTPJ, from $0.2-0.5 \mathrm{~s}$ post-stimulus onset, suggesting that the $\mathrm{ACC}$ and lateral PFC contribute later to the process of perspective-taking. We also found that these two regions displayed directed functional connectivity to the rTPJ, mediated by low frequency brain rhythms $(1-10 \mathrm{~Hz})$, indicative of top-down processing (Von Stein, Chiang, \& König, 2000).

Activity within the ACC and lateral PFC is typically associated with cognitive control (Aron, Robbins, \& Poldrack, 2014) and conflict monitoring (Botvinick, Cohen, \& Carter, 2004). However recent work has shown the regions to be also implicated in a number of theory of mind studies (Hartwright, Apperly, \& Hansen, 2012, 2015; Samson, Houthuys, \& Humphreys, 2015; Vogeley et al., 2001). Activity within this context has been argued to reflect the detection (Amodio \& Frith, 2006; Lieberman, 2007) (ACC) and resolution (Hartwright et al., 2012; Samson, Apperly, Kathirgamanathan, \& Humphreys, 2005) (lateral PFC) of conflict between self and other perspectives (Hartwright et al. 2016a). We therefore propose, see Figure 6.14, that ACC-TPJ and PFC-TPJ connectivity during later stages of perspective-taking, reflects domain-general "top-down" executive control processes (Duncan \& Owen, 2000) required for suppressing the self-perspective, in favour of taking the other's perspective (Hartwright et al., 2015; Samson et al., 2005; Van der Meer, Groenewold, Nolen, Pijnenborg, \& Aleman, 2011), and/or for controlling the conflict between the physical self and the transformed self (the "other") (May, 2004; Santiesteban et al., 2012; Wang et al., 2016). Whilst the involvement of executive-control processes in perspective-taking is based on substantial empirical research (Aron et al. 2014; Hartwright et al. 2016a), to avoid reverse-inference, future work could vary executive demands during perspective-taking (Bradford, Jentzsch, \& Gomez, 2015), in combination with brain stimulation (Wang et al., 2016) to establish the causal role of the lateral PFC and rACC. 


\section{Executive Network:} Top-down Cognitive Control

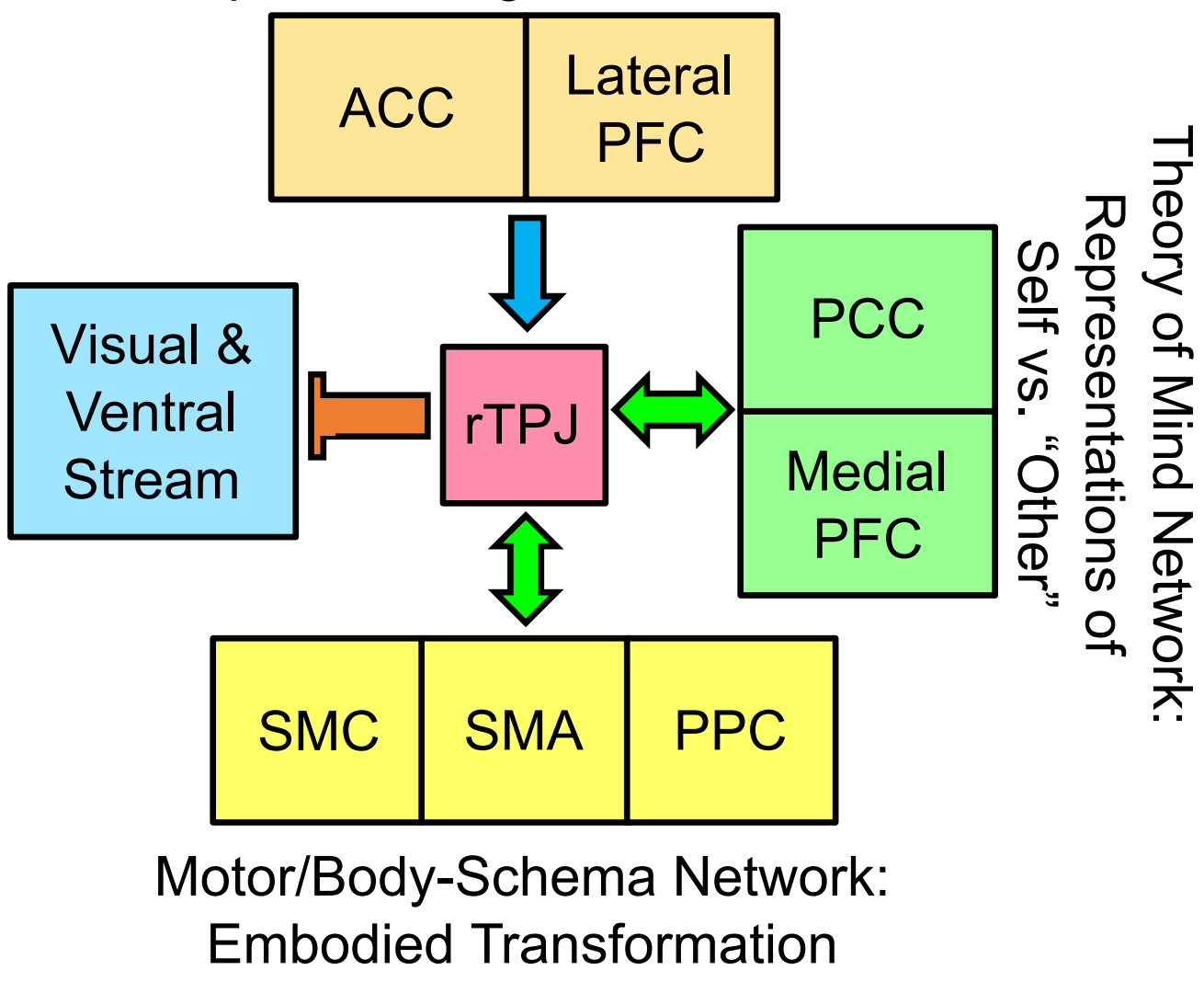

Figure 6.14. Proposed network underlying embodied perspective-taking in the neurotypical brain, linked by power and phase in the theta-band (3-7Hz). During initiation of perspective-taking behaviour, early rTPJ activity co-ordinates connectivity decreases with visual regions, whilst increasing connectivity with regions involved in Theory of Mind, and Motor/Body-Schema. Increases in low frequency (primarily theta) power within the lateral PFC and ACC reflect domain-general cognitive control processes for detecting and managing top-down the conflict between self and other perspectives.

\subsubsection{Neural Basis of Perspective-Taking in Autism Spectrum Disorder (ASD)}

In Experiment 2 we investigated ASD-related individual differences in embodied perspective-taking. Behaviourally, the ASD group had longer reaction times compared with age-matched controls, specifically in the LR-160 condition (Figure 6.7). This supports the idea of a selective impairment in embodied perspective-taking in ASD, i.e. level 2 , rather than perspective-tracking, i.e. level 1 (Pearson et al., 2013). Having said this, we did not find a significant correlation between the angle of disparity (LR-160 vs LR-60 reaction time) and $A Q$ score. Autistic symptoms might be better assessed using a clinical assessment (e.g. the ADOS), rather than a self-report questionnaire. Using MEG, we showed that when increasing the angle of disparity between self and other perspectives, 
controls had increased theta-band $(4-7 \mathrm{~Hz})$ power compared with the ASD group, within ventral visual cortex, right temporal cortex and lateral PFC. However when quantifying the same angle of disparity for the alpha-band, the ASD group showed greater desynchronisation over bilateral occipital cortex spreading into the ventral visual stream. Finally, using Granger causality between four regions of interest during LR-160 trials, it was found that the control group had increased feedforward connectivity from V1-to-V5 and decreased feedback connectivity from PFC-to-V5, mediated by theta-band oscillations.

The difference in oscillatory signatures reported between groups, i.e. theta versus alpha, may reflect the differences in cognitive strategies employed during the task (Pearson et al., 2016, 2013). For controls, the increase in theta-band oscillations was also reported in Wang et al., (2016) and Experiment 1. Theta localised to ventral occipital, temporal cortex and lateral PFC. Surprisingly, there was no significant increase in theta-power over rTPJ. This might due to differences in the age between participants in Experiments $1 \& 2$, reduced numbers of trials in Experiment 2, as well as the predominantly male sample, which may have reduced effect sizes and group differences. Replicating Experiment 1, there was an increase theta-band power for lateral PFC, suggesting that controls use executive control processes during embodied rotation of the self. In support of this, we found evidence for top-down connectivity from PFC-to-rTPJ in controls (Experiment 1), mediated by theta-band oscillations. As argued above, this may reflect the management of the conflict between self and other perspectives, which has been shown to be affected in autism (Conson et al., 2015).

The ASD group showed a very different pattern of brain activity during perspective-taking, with increasing angular disparity associated with alpha-band desynchronisation, within visual cortex. We suggest this may reflect a mental rotation (MR) strategy rather than an embodied egocentric transformation strategy, previously reported behaviourally (Conson et al., 2015; Pearson et al., 2016). Posterior alpha power desynchronisation is thought to reflect reduced functional inhibition during visual processing, associated with the emergence of high-frequency gamma-band oscillations and neural spiking (Jensen \& Mazaheri, 2010). This suggests that, unlike controls, the ASD group relied on brain regions in the early and ventral visual cortex rather than the extended network of temporoparietal and pre-frontal regions required for embodied transformation (Samson et al., 2012). Interestingly, alpha-band oscillations have been causally linked with mental rotation abilities. Klimesch, Sauseng, \& Gerloff (2003) showed that repetitive TMS administered at alpha-frequencies, improved performance during a mental rotation task. A follow-up 
neurofeedback study showed that participants who were able to modulate alpha-band power, improved their performance on a mental rotation task (Hanslmayr, Sauseng, Doppelmayr, Schabus, \& Klimesch, 2005). During perspective-taking, alpha desynchronisation may therefore be a marker of an effortful and slower MR strategy, whereas theta is associated with the faster and more automatic embodied strategy in controls (Wang et al., 2016). Future research could investigate this further by characterising the neural dynamics of embodied processing and mental rotation within the same participants. Repetitive TMS entrainment could also be used to investigate the causal role of alpha vs theta oscillations as a function of neurocognitive strategy during perspective taking (Gooding-Williams, Wang, \& Kessler, 2017).

Quantifying differences between four regions of interest, it was found that there were decreases in both feedforward and feedback connectivity for the ASD group. The decrease in connectivity from lateral PFC to V5, mediated by theta-band oscillations, suggests reduced top-down, executive control over embodied processes in ASD (Conson et al., 2015). This would be consistent with the role of frontal theta oscillations in cognitive control processes (Cavanagh \& Frank, 2014). Our results also support previous MEG research, showing global hypoconnectivity in ASD, mediated by low-frequency oscillations (Doesburg, Vidal, \& Taylor, 2013; Khan et al., 2013), see Chapter 1 and Kessler, Seymour, \& Rippon (2016). Interestingly, we found reduced feedforward connectivity for the ASD group from V1-to-V5, again mediated by theta-band oscillations. One previous study reported increased feedforward connectivity in autism during somatosensory task, but these effects were confined to the gamma-band (Khan et al., 2015). Recently it has been shown that theta oscillations in visual cortex are predominantly feedforward in nature, and reduced by selective attention (Spyropoulos, Bosman, \& Fries, 2018). Therefore the reduction in theta GC values may result from ASD participants increasing selective attention to the stimuli during difficult LR-160 trials in order to facilitate a mental rotation strategy, to a greater extent than controls. Clearly, additional research is required to investigate how low-level visual processes interact with higher-level perspective-taking and social processes in autistic populations (Palmer, Seth, \& Hohwy, 2015).

More generally, our results show that when instructed to imagine the world from another's perspective, ASD participants activate visual regions (Samson, 2012), rather than the extended network of regions supporting embodied transformation, centring on the areas around the right TPJ and in PFC. A bias towards prioritising bottom-up sensory information, at the expense of top-down prior information, has been described by predictive-coding accounts of autism (Kessler, Seymour, \& Rippon, 2016; Palmer, 
Lawson, \& Hohwy, 2017). These generally focus on sensory aspects of ASD, but can be easily extended for social and embodied processes. Just as one can represent an object for perceptual inference, one can also represent another's perspective, or mental state, if it helps to predict the causes of sensory input and minimise prediction error (Palmer, Seth, \& Hohwy, 2015). Embodied processing may be particularly affected in ASD, as another's perspective is causally inferred and richly context-sensitive, sitting towards the top of the cortical hierarchy. Our findings therefore hint at the intriguing possibility of a common mechanism underlying both sensory and social symptoms in ASD. During development, impaired embodied abilities may result in cascading deficits in social cognitive skills more generally (e.g. Theory of Mind) (Kessler, Seymour, \& Rippon, 2016; Pearson, Ropar, \& Hamilton, 2013). Whilst the paradigm in this study required a relatively simple non-social judgement (left/right), there is evidence to suggest that visual perspective-taking performance can predict theory of mind in children (Hamilton, Brindley, \& Frith, 2009). For perspective-taking, ASD participants can adopt a compensatory mental rotation (MR) strategy, potentially supported by increased alpha desynchronisation over visual cortex. However, as tasks become more abstract, for example false belief tasks requiring representing what others are thinking, this strategy becomes inefficient, if not impossible.

\subsubsection{Limitations}

We note two limitations. First, whilst sensor-level analysis across Experiments 1 and 2 revealed effects in the theta $(3-7 \mathrm{~Hz})$ and alpha $(8-12 \mathrm{~Hz})$ bands, inspection of timefrequency responses at the source level suggests that these effects might spread into lowfrequency delta frequency ranges $(1-3 \mathrm{~Hz})$. Furthermore, granger causality results from lateral PFC to right TPJ across Experiments 1 and 2, show effects in the delta and theta bands. It should be noted that the fine-grained definition of frequency ranges is confounded by spectral smoothing applied during the frequency decomposition process. Future work should therefore attempt to clarify whether there might be additional independent or multiplexed oscillatory networks (e.g. Seymour et al. 2017) at different frequency ranges, including those between delta/theta and gamma $(>30 \mathrm{~Hz})$ bands (CaO et al., 2018). Nevertheless, this chapter suggests that theta $(3-6 \mathrm{~Hz})$, for neurotypicals, and alpha $(8-12 \mathrm{~Hz})$ for the ASD group, appear to be the most dominant oscillatory frequency bands, underlying perspective-taking. Second, in Experiment 2, we did not collect a formal clinical assessment of autism, e.g. the Autism Diagnostic Observation Schedule. Strict participant exclusion criteria were therefore implemented, and only autistic participants with a confirmed clinical diagnosis of ASD or Asperger's syndrome were included in the study. Between groups, there were significant differences in autistic and sensory traits, see Table 6.1. 


\section{Author Contributions}

RS, KK \& GR co-designed the study. RS collected the data, carried out the analysis, organised the data and code for sharing and was the primary author for this chapter. GGW helped with data collection. HW gave data analysis guidance.

\section{Conflict of Interests Statement}

The authors wish to declare the research was conducted in the absence of any commercial or financial relationships that could be construed as a potential conflict of interest.

\section{Acknowledgements}

We wish to thank: the volunteers who gave their time to participate in this study; and the Wellcome Trust, Dr Hadwen Trust and Tommy's Fund for supporting research costs.

Robert Seymour was supported by a cotutelle PhD studentship from Aston University and Macquarie University. 


\section{Chapter 7: General Discussion}

In this chapter, the main results of the thesis are summarised, and limitations are considered. I conclude by discussing the implications of my findings within a broader theoretical context.

\subsection{Summary of Main Findings}

\subsubsection{A Robust Pipeline for Phase Amplitude Coupling (PAC)}

In Chapter 2, we investigated the various algorithms and analysis steps involved in detecting PAC for neurophysiological data, and presented an openly available analysis pipeline. We compared four different algorithms using this pipeline to analyse an MEG dataset from 16 adult participants performing an interactive task, with embedded visual grating. PAC values were statistically compared with a baseline period (no visual grating). It was found that the MVL-MI approach from Canolty et al., (2006) was biased by the amplitude (power) of high-frequency oscillations. This was not the case for the MVL-MI approach from Özkurt \& Schnitzler (2011) and PLV-MI approach from Cohen (2008), both of which showed significant increases in alpha-gamma PAC. However, the KL-MI approach from Tort, Komorowski, Eichenbaum, \& Kopell (2010a) did not show increases in alpha-gamma PAC, potentially due to this approach being more conservative. Finally, we validated our PAC analysis pipeline using simulated data and ruled out the possibility that our findings were driven by non-sinusoidal oscillations. These results informed our PAC analysis in Chapter 3, where we opted to use the MVL-MI approach from Özkurt \& Schnitzler (2011) and PLV-MI approach from Cohen (2008).

Whilst Chapter 2 focussed on four of the most common PAC algorithms, there has been recent progress in the development of new PAC metrics (e.g. Cheng, Li, Wang, Wang, \& Zhang, 2018; La Tour et al., 2017; Martínez-Cancino et al., 2019; Nadalin et al., 2019). Once these new methods have been empirically verified, they could potentially provide new insights into cross-frequency coupling in the human brain, and inform future research in relation to PAC in ASD.

\subsubsection{Reduced Phase Amplitude Coupling in ASD}

In Chapter 3, we investigated alpha-gamma PAC during visual processing in ASD using the pipeline developed in Chapter 2. MEG data were collected from a group of 18 adolescents diagnosed with ASD and 18 age-matched controls whilst participants performed an interactive task, with embedded visual grating. Compared with baseline, 
PAC values for the control group showed increases in alpha-gamma PAC for primary visual cortex, $\mathrm{V} 1$, between $7-9 \mathrm{~Hz}$ phase and $50-80 \mathrm{~Hz}$ amplitude. In comparison, the ASD group showed significantly reduced alpha-gamma PAC for $\mathrm{V} 1$ between $8-9 \mathrm{~Hz}$ phase and 52-74Hz amplitude.

\subsubsection{Reduced Steady-State Responses in ASD}

In Chapter 4, we investigated auditory steady-state responses (ASSRs) in ASD, using a clicktrain stimulus to entrain neural activity in the auditory system at $40 \mathrm{~Hz}$. Previous research has found reductions in ASSRs for autistic participants and the first-person relatives of people with ASD (Rojas et al., 2011; Wilson, Rojas, Reite, Teale, \& Rogers, 2007). Using MEG, we attempted to replicate and extend these findings in a group of 18 adolescent ASD participants and 18 age-matched controls. The ASD group showed reductions in oscillatory power and inter-trial coherence at $40 \mathrm{~Hz}$, within bilateral auditory cortex. Interestingly, differences in inter-trial coherence emerged around $0.5-1.0$ s postclicktrain onset, suggesting that disruptions to gamma-band entrainment in ASD are more pronounced during sustained auditory stimulation. To investigate the developmental trajectories of ASSRs we performed a median split by age, separating the data into a group of older adolescents (age: 17-20) and younger adolescents (age: 14-17). For controls, there was an increase in $40 \mathrm{~Hz}$ gamma power for older versus younger adolescents. This was not the case for the ASD group. Finally, we found that unlike ASSRs, evoked gamma responses (tGBR) to the auditory clicktrain were not different between ASD and control groups.

\subsubsection{Feedforward vs Feedback Connectivity in ASD}

Returning to the results of Chapter 3, we investigated inter-regional (V1-V4) feedforward and feedback connectivity. Recent work using ECOG in macaques and MEG in humans has established that the visual system operates via distinct oscillatory communication channels, with gamma oscillations mediating feedforward connectivity and alpha oscillations mediating feedback connectivity (Bastos et al., 2015; Michalareas et al., 2016). Using the visual-grating MEG data from 18 ASD and 18 controls, directed functional connectivity was quantified between visual areas $\mathrm{V} 1$ and $\mathrm{V} 4$, using nonparametric spectrally resolved Granger causality (Dhamala, Rangarajan, \& Ding, 2008). For the control group, we found that V1-to-V4 feedforward connectivity was accompanied by increased gamma-band GC; whereas V4-to-V1 feedback connectivity was accompanied by increased alpha-band GC. The ASD group showed comparable increases in gamma feedforward GC, but significantly reduced V4-to-V1 alpha-band connectivity, compared with controls. This decrease in feedback connectivity was positively associated with the level of autistic traits reported by ASD participants. 


\subsubsection{Predictive-Coding in ASD}

In Chapter 5, we investigated the neural basis of predictive-coding in ASD using an auditory oddball paradigm (Recasens \& Uhlhaas, 2017). MEG data was collected from 16 adolescents diagnosed with ASD and 16 age-matched controls. It was found that evoked responses to the unexpected omission of an auditory tone were reduced in the ASD group, around $0.1 \mathrm{~s}$, in bilateral superior-temporal gyrus. In contrast, evoked responses to an unexpected duration deviant tone were equivalent between groups. This provides evidence for reduced top-down prediction in ASD, rather than reduced neural adaptation, consistent with "hypo-prior" accounts of ASD (Friston, Lawson, \& Frith, 2013; Pellicano \& Burr, 2012). We also tested precision-weighting theories of ASD, by presenting auditory tones in silence versus babble-noise (Dreschler, Verschuure, Ludvigsen, \& Westermann, 2001). However, for the babble-noise condition, no evoked mismatch responses were found for either group. It therefore remains unclear whether ASD is associated with reductions in top-down predictions or rather the atypical weighting of predictions (Palmer, Lawson, \& Hohwy, 2017).

\subsubsection{Embodied Perspective-Taking in ASD}

In Chapter 6, we investigated the neural basis of perspective-taking, using a paradigm which dissociates perspective-taking from perspective-tracking (Kessler \& Rutherford, 2010; Surtees, Apperly, \& Samson, 2013). In Experiment 1 we collected MEG data from 18 adults aged 23-45. During perspective-taking, increasing the angle of disparity between self and other perspectives was accompanied by increased theta-band $(4-7 \mathrm{~Hz})$ power for the right temporoparietal junction (rTPJ), pre-frontal cortex and anterior cingulate cortex. Similar increases in theta power have been reported using the same paradigm (Wang, Callaghan, Gooding-Williams, McAllister, \& Kessler, 2016). We expanded on these findings by quantifying theta-band phase synchrony between the rTPJ, and the rest of the cortex during perspective-taking. It was found that the rTPJ increased its coupling with regions involved in theory of mind and regions coding for motor/body schema, providing evidence for the embodied nature of perspective-taking. In addition, we estimated directed functional connectivity between regions of interest, using Granger causality. There was significantly increased GC between the pre-frontal cortex $(1-5 \mathrm{~Hz})$ and anterior cingulate cortex $(1-10 \mathrm{~Hz})$ to the rTPJ. These connections were argued to reflect domain-general cognitive control processes for detecting and managing, in a top-down manner, the conflict between self and other perspectives (Hartwright, Hansen, \& Apperly, 2016; Ramsey, Hansen, Apperly, \& Samson, 2013). 
In Experiment 2, the same paradigm and analysis pipeline was used to compare a group of 18 ASD participants and 17 typically developing controls. Behaviourally, the ASD group were slower during perspective-taking trials, where an avatar was rotated 160 away from the egocentric perspective. This increased angle of disparity between self and other perspectives was accompanied by different changes in oscillatory power between groups. As for experiment 1, the control group showed increases in theta-power $(4-7 \mathrm{~Hz})$ which were localised in source space to higher visual and pre-frontal regions. In contrast, the ASD group showed greater alpha-power $(8-12 \mathrm{~Hz})$ desynchronisation which localised to visual cortex. This result was interpreted as evidence that, unlike controls, ASD participants did not use an embodied egocentric transformation to achieve perspectivetaking. Instead, we suggest that ASD participants might prefer a mental rotation strategy, i.e. rotating the avatar towards their own perspective, which results in increased visual activity compared to control subjects. Finally, directed functional connectivity was measured between regions of interest using Granger causality. It was found that the ASD group displayed reduced feedback connectivity from pre-frontal cortex to visual area V5, compared with controls, as well as reduced feedforward connectivity between visual areas V1 and V5.

\subsubsection{ASD and MEG}

It is important to note that the data presented in this thesis were acquired using MEG, which offers certain advantages over other neuroimaging techniques. MEG has greater temporal resolution than $\mathrm{fMRI}$, allowing the measurement of oscillatory power and phase synchrony. Compared with EEG recordings, MEG offers more accurate source localisation and higher signal to noise ratios. This thesis clearly demonstrates that by utilising the excellent spatiotemporal properties of MEG recordings, combined with novel analysis techniques (Chapter 2), neuroimaging research can provide insights into the neural basis of ASD (Chapters 3-6).

\subsection{Limitations}

We note several limitations to the research presented in this thesis. First, we did not collect a formal clinical assessment of autism, e.g. the ADOS. However, strict participant exclusion criteria were applied: autistic participants were only included with a confirmed clinical diagnosis of ASD or Asperger's syndrome (APA, 2013). For data presented in Chapters 3-5, there were significant differences in autistic and sensory traits between ASD and control groups, measured using two self-report questionnaires. Future work should collect a wider range of clinical assessments to investigate in depth how oscillatory connectivity relates to a wider range of clinical ASD symptoms. 
On a related note, in Chapters 3 and 4, we could not find an association between the oscillatory connectivity in ASD participants and their sensory symptoms as reported via the Glasgow Sensory Questionnaire, GSQ (Robertson \& Simmons, 2013). This goes against the ideas outlined in Chapter 1 , where local dysregulation was argued to underlie these sensory symptoms. Upon closer inspection of GSQ data, the ASD participants reported a mixture of hyper- and hypo-sensitive traits between different sensory modalities, see Figure 7.1. When aggregating this complex, multisensory data into a "total" GSQ score, precise brain-behavioural correlations will be problematic. In addition, with only 18 useable ASD datasets, our GSQ-connectivity correlation analyses could well be underpowered (Rousselet \& Pernet, 2012). Brain-behaviour relationships might be better assessed using psychophysical tests of visual/auditory perception (Ashwin et al., 2009), combined with formal clinical assessments, rather than self-report questionnaires with multiple dependent variables.

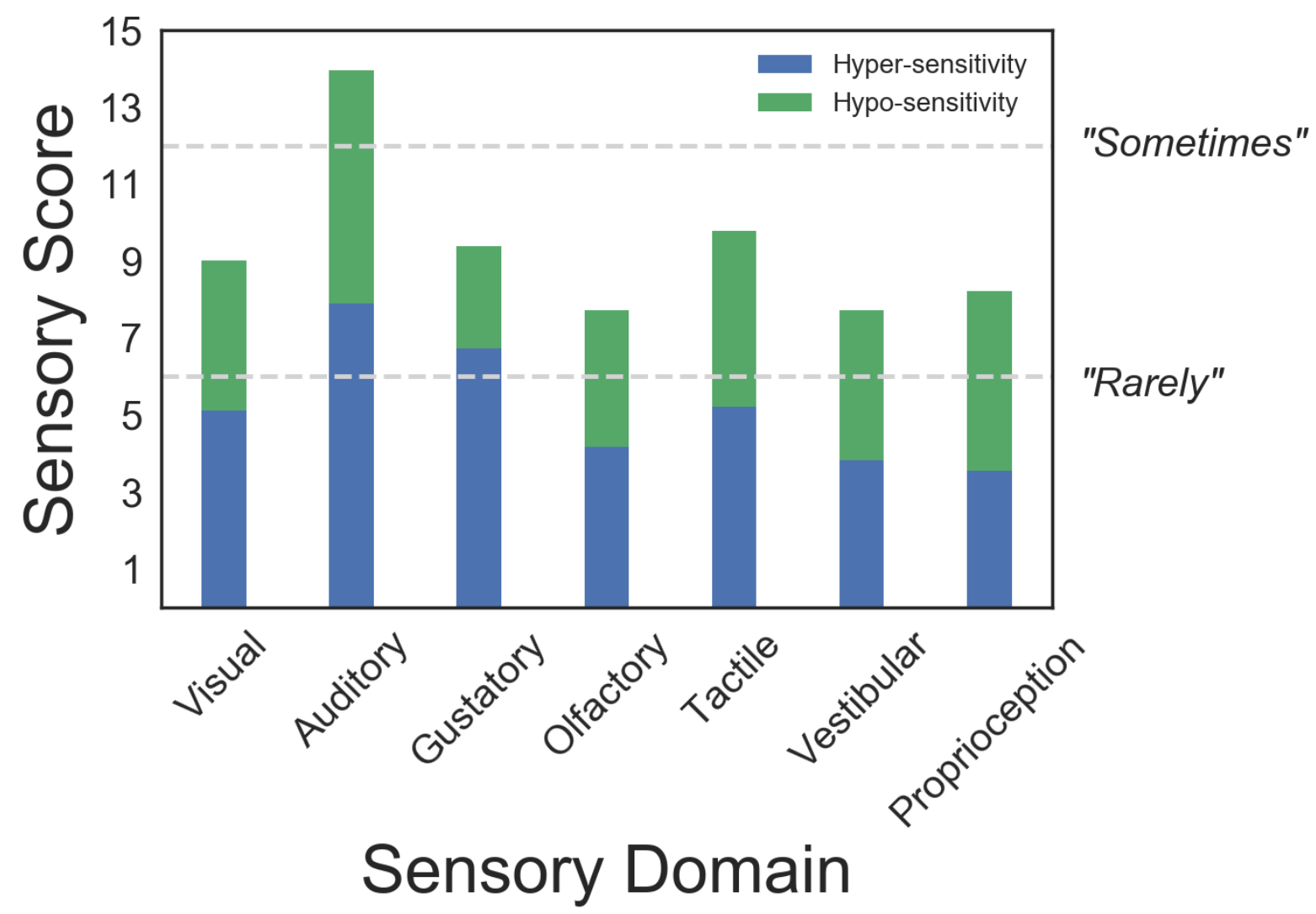

Figure 7.1: Responses to the Glasgow Sensory Questionnaire were grouped by sensory domain (maximum score $=20$ ) and hypo- / hyper-sensitivity (green and blue bars respectively). Our data show a heterogeneous pattern of sensory symptoms, with mixture of hypo- and hyper-sensitivities. Visual symptoms scored 9.0/20 corresponding to questionnaire answers between "Rarely" and "Sometimes". Auditory sensory symptoms were higher than for other modalities. 
Thirdly, it should be noted that ASD is a lifelong neurodevelopmental disorder, with sensory and social symptoms emerging during early childhood, and persisting into adolescence and adulthood (APA, 2013; Nomi \& Uddin, 2015). This thesis has primarily focussed on characterising oscillopathies in adolescent participants: ASD data was collected from participants aged 10-20. One advantage of studying adolescents over younger children, is that high-quality neuroimaging data can be acquired more easily, and a greater number of experimental trials can be included before participants become fatigued. This is especially important for connectivity metrics which are sensitive to differences in signal-to-noise ratio between groups (Bastos \& Schoffelen, 2016). We acknowledge that additional research is needed to investigate whether the findings reported in this thesis (differences in phase-amplitude coupling, feedforward/feedback connectivity and predictive coding) extend across a wider age-range of autistic participants. The paradigms used in Chapters 3-5 could easily be used for younger participants (e.g. aged upwards of 3 ) in combination with custom child-sized MEG systems (Johnson, Crain, Thornton, Tesan, \& Reid, 2010; Roberts et al., 2014). Additionally, longitudinal studies are crucial for establishing the developmental trajectories of oscillopathies in ASD (e.g. Loth et al., 2017).

Fourth, to study the directed functional connectivity between regions of interest (Chapters 3,5 and 6), this thesis utilised non-parametric Granger causality, GC (Dhamala et al., 2008). GC can be operationalised in the frequency domain, thereby providing information about the direction of connectivity, and the frequency band(s) underlying this (Mingzhou, Chen Yonghong, \& Bressler 2006). Furthermore, it has been argued that GC is not biased by issues associated with volume conduction (Michalareas et al., 2016). However, measures of GC are susceptible to the common input problem: where increased $G C$ is observed between regions $A$ and $B$, this could be driven by a common input from another region $\mathrm{C}$, see Figure 7.2. In Chapter 3 , the reported V1-V4 connectivity could conceivably be driven by a common input to visual areas, for example from the thalamus (Horincz, Kékesi, Juhász, Crunelli, \& Hughes, 2009). Whilst, we cannot entirely rule out this possibility, it should be noted that direct laminar-dependent V1-V4 cortical connectivity has been observed using retrograde tracing in macaques (Barone, Batardiere, Knoblauch, \& Kennedy, 2000; Markov et al., 2014). Furthermore, Kerkoerle et al., (2014) report that in macaques, electrical stimulation of V4 results in increased alpha-activity within V1, whereas stimulation of V1 results in increased gamma within V4. Finally, it is hard to conceive of how a common input alternative could fully explain the observed asymmetries between feedforward and feedback GC connectivity across frequencies. 


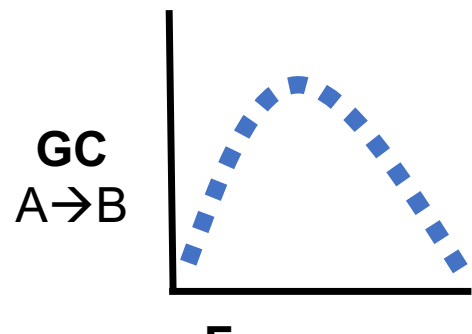

Frequency

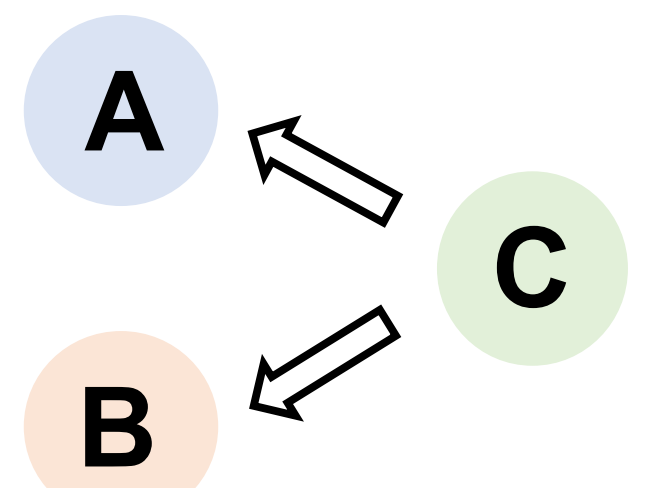

Figure 7.2 The common input problem. Increased Granger causality (GC) values are observed between regions $A$ and $B$ due to common input from region $C$, rather than a direct connection between regions $A$ and $B$.

Finally, the findings reported in this thesis come from a relatively low number of participants diagnosed with ASD (18 participants in Chapters 3,4,6; and 16 participants in Chapter 5). Our findings should therefore be treated with caution and may not generalise to the entire ASD spectrum. The relatively low participant numbers may have contributed to inadequate statistical power in some instances, for example the non-significant brainbehaviour correlations reported in Chapters 3,4 and 6. Additionally, the developmental analysis presented in Chapter 4 Figure 4.7 (where sub-groups contained only 9 participants) needs to be replicated and extended using a larger sample. Nonetheless, the novel analysis approaches presented throughout this thesis have identified interesting differences between ASD and control participants, many of which were hypothesied based on previous findings (see Chapter 1 and Kessler et al., 2016). This thesis therefore provides important pilot findings for future research, which should include greater numbers of participants and detailed clinical assessments.

\subsection{Implications and future directions}

Overall, our findings provide evidence for atypical cortical activity in ASD, as measured by magnetoencephalography (MEG). Results are consistent with the view that brain oscillations in ASD are characterised by local dysregulation and global hypoconnectivity.

\subsubsection{Gamma-band Oscillopathies in ASD}

In Chapter 1, previous findings in relation to gamma oscillations in ASD were reviewed. Based on the heterogeneity of the literature reviewed, we argued that findings were consistent with the view of dysregulated local gamma activity, driven by an underlying excitation-inhibition imbalance (Kessler, Seymour, \& Rippon, 2016; Simon \& Wallace, 
2016). One oscillatory mechanism associated with the regulation of gamma-band activity is phase-amplitude coupling (PAC) - a form of cross-frequency coupling in which the phase of a low-frequency oscillation is coupled to the amplitude of a high-frequency oscillation, usually gamma (Canolty et al., 2006; Canolty \& Knight, 2010; Jensen \& Colgin, 2007). PAC has been proposed to act as a mechanism for the dynamic coordination of brain activity over multiple spatial scales, with the amplitude of high-frequency gammaband activity within local ensembles coupled to large-scale patterns of low-frequency phase synchrony (Bonnefond, Kastner, \& Jensen, 2017).

Our finding of reduced alpha-gamma phase amplitude coupling in ASD (Chapter 3) supports the notion that local gamma activity is dysregulated in ASD. Similar findings of reduced alpha-gamma PAC have previously been reported in ASD populations for emotional face stimuli (Khan et al., 2013) and at rest (Berman et al., 2015). PAC is thought to depend on interactions between excitatory and inhibitory populations of neurons, as well as coupling between superficial and supragranular layers of cortex (Mejias, Murray, Kennedy, \& Wang, 2016; Onslow, Jones, \& Bogacz, 2014; Spaak, Bonnefond, Maier, Leopold, \& Jensen, 2012). Therefore, findings of reduced PAC link with neurophysiological models of an E-I imbalance in ASD at the cellular level (Rubenstein \& Merzenich, 2003).

It is also important to note that reductions in PAC arose despite similar changes in gamma and alpha power between ASD and control groups - a finding similar to that of Khan et al., (2013). This suggest that local gamma activity can be dysregulated without a discernible change in power. Future ASD-MEG research should therefore explore the precise regulation of gamma oscillations via cross-frequency coupling, rather than relying on measures of power alone. Previously published datasets could also be reanalysed, using the PAC pipeline outlined in Chapter 2.

Where local connectivity is affected, it has been argued that this would have knock-on consequences for the development of inter-regional, and potentially global, connectivity in ASD (Kessler et al., 2016; Rubenstein \& Merzenich, 2003). In Chapter 3, we found that local PAC in V1 was correlated with inter-regional feedback connectivity (however please note that this correlation was uncorrected for multiple comparisons). This hints at the intriguing possibility that where local activity in primary visual cortex is dysregulated, global patterns of feedback connectivity are concurrently affected in ASD (Khan et al., 2013). As mentioned in Chapter 1 , this effect could be bi-directional: reduced top-down connectivity may be causally related to dysregulated local activity and vice versa (Kessler et al., 2016; Khan et al., 2013). From a predictive-coding perspective, reduced local-global 
connectivity would result in disruptions to the integration of top-down predictions with bottom-up sensory information during sensory processing (Kessler et al., 2016; Pellicano \& Burr, 2012). This integration of information is particularly important where top-down predictions help to resolve ambiguous bottom-up information, for example where it is hard to separate signal from noise (Mamashli et al., 2017; Van de Cruys, Van der Hallen, \& Wagemans, 2017). To investigate links between local and inter-regional connectivity in ASD, future work could utilise the methods outlined in Chapter 3 , in combination with carefully designed paradigms which vary bottom-up versus top-down processing during visual perception. Potential ideas are discussed further in Box 7.1.

In Chapter 4, a reduction in auditory steady state responses (ASSRs) at $40 \mathrm{~Hz}$ was found for the ASD compared with the control group, in terms of power and inter-trial coherence. Steady-state responses originate from primary auditory cortex, are sustained for the duration of the stimulus, and are generated through non-linear neural dynamics within local circuits (Pantev, Roberts, Elbert, Ro $\beta$, \& Wienbruch, 1996; Rippon, Brock, Brown, \& Boucher, 2007; Rojas \& Wilson, 2014). Recent findings suggest that the ASSR power increases with age, up to 14-16 years (Cho et al., 2015; Edgar et al., 2016), in line with the developmental role of neural pruning during adolescence and the late development of inhibitory GABAergic interneurons (Fuhrmann, Knoll, \& Blakemore, 2015; Gao, Newman, Wormington, \& Pallas, 1999). Auditory processing in ASD could therefore be affected by delays to the maturation of these neurobiological processes, resulting in dysfunctional mechanisms for the generation of non-linear local gamma-band responses. This idea is paralleled by Khan et al., (2015), who, based on somatosensory data, suggested that local activity in ASD is characterised by specific disruptions to non-linear aspects of cortical processing. Again, this could conceivably reflect an underlying imbalance between excitatory and inhibitory populations of neurons (Casanova, Buxhoeveden, \& Gomez, 2003). In Chapter 4 , we also found that there were no differences between ASD and control groups in terms of broadband evoked gamma power and synchrony. This highlights the importance of measuring early evoked gamma alongside sustained and induced gamma power in ASD populations (Rojas et al., 2011). Potential gamma oscillopathies in ASD are likely to be dependent upon time-period under investigation and the type of gamma generated by the stimulus (evoked, induced, entrained etc.). It should be noted that some studies using auditory tones have reported decreases in evoked gamma for ASD populations (Edgar et al., 2015; Gandal et al., 2010; McFadden, Hepburn, Winterrowd, Schmidt, \& Rojas, 2012; Port et al., 2016). Future work should therefore seek to systematically investigate which types of auditory stimuli (e.g. tones, clicktrains, speech, natural sounds) lead to ASD-related oscillopathies in evoked versus induced/entrained gamma power. 
More generally, whilst this thesis has focussed on gamma-band oscillations measured via M/EEG, our findings would be complimented by additional measures relating to neuronal excitation-inhibition interactions (Dickinson et al., 2016). The power of gamma oscillations is linked, either directly or indirectly, to the concentration of the neurotransmitter GABA (Muthukumaraswamy et al., 2009), which plays a major inhibitory role in canonical microcircuits (Markram et al., 2004). The concentration of GABA, and other neurotransmitters in the brain, can be estimated non-invasively using a technique called magnetic resonance spectroscopy (Ross \& Bluml, 2001; MRS). Interestingly, a recent MRS study showed a reduction in the concentration of GABA for ASD participants relative to control participants, implying a disruption to the E-I balance (Gaetz et al., 2014). It would be particularly interesting to link GABA concentration, measured through MRS, with gamma band power and PAC in a cohort of ASD and control participants.

Pharmacological interventions designed at modulating cortical inhibition (e.g. GABA agonists) could also be used to provide causal evidence for an E-I imbalance in ASD, as well as act as a potential therapeutic intervention (Politte, Charles \& McDougle, 2014). Additionally, neuronal excitability in ASD could be investigated through concurrent TMSEEG, which aims to stimulate the brain using TMS and measure the resulting evoked potential(s) or changes to oscillatory connectivity using EEG (Chung et al., 2015). These TMS-evoked potentials are argued to be markers of cortical E-I processes. For example the N100 component of motor-evoked potential has been reliably linked with cortical inhibitory processes (Farzan et al., 2013). Interestingly, one study used concurrent TMSEEG to stimulate the dorsolateral prefrontal cortex, primary motor cortex, and temporoparietal junction in a group of ASD and controls participants (Kirkovski et al., 2016). However, there were no group differences in oscillatory power or connectivity as measured with EEG, potentially due to the older age-group under investigation. TMS can also be used to entrain certain neuronal oscillations, i.e. by stimulating at a certain rhythmical frequency. This technique therefore has great potential in providing causal evidence for the role of E-I related oscillopathies in ASD symptomatology (Kessler et al., 2016).

\subsubsection{Feedforward vs Feedback Connectivity in ASD}

Previous M/EEG research has found reductions in low-frequency phase-synchrony for autistic populations across a range of cognitive paradigms, and in the resting-state (Doesburg, Vidal, \& Taylor, 2013; Kitzbichler et al., 2015), reviewed in Section 1.4.3. However, as argued in Chapter 1, to fully understand low-frequency oscillopathies in ASD, it is important to establish the direction of connectivity under investigation (Khan et al., 2015). 
In Chapter 3, building on previous ECOG/MEG research (Bastos et al., 2015; Michalareas et al., 2016), we used non-parametric Granger causality to investigate the direction of visual inter-regional (V1-V4) connectivity in ASD and control groups (Dhamala et al., 2008). For controls, V1-to-V4 feedforward connectivity was mediated by gamma oscillations; whereas V4-to-V1 feedback connectivity was mediated by alpha oscillations. This dissociation between feedforward-gamma and feedback alpha oscillations replicates recent work in macaques and humans (Bastos et al., 2015; Kerkoerle et al., 2014; Michalareas et al., 2016). Compared with controls, the ASD group showed reductions in feedback-alpha connectivity but no difference in feedforward-gamma connectivity, as hypothesised in Chapter 1. Our results therefore suggest that the frequency-specific, hierarchical organisation of the visual system is affected in ASD.

These findings link with emerging theories of typical perception. Predictive-coding accounts of cortical activity describe the passage of top-down predictions from higher to lower areas via feedback pathways, with prediction errors computed at each level of the hierarchy being passed forward via feedforward pathways (Friston, 2005). Predictivecoding accounts of autism suggest that differences in perception emerge from fewer or hyper-precise top-down predictions, such that perception is less influenced by prior knowledge and contextual cues (Palmer et al., 2017; Pellicano \& Burr, 2012). Our data clearly support this proposal by showing reduced feedback connectivity in the visual cortex in autism. See Section 7.3.3 for more discussion on this.

Reductions to feedback connectivity and dysfunctional mechanisms of predictive coding, would have knock-on consequences for perceptual processes in ASD (Van Boxtel \& Lu, 2013). Feedback connectivity within the visual system is thought to provide a modulating influence, linked with processes such as attention, top-down predictions, and global processing (Engel, Fries, \& Singer, 2001; Kafaligonul, Breitmeyer, \& Öğmen, 2015; Manita et al., 2015; Zhang et al., 2014). It is precisely these aspects of visual processing, which are affected in ASD (Dakin \& Frith, 2005; Robertson \& Baron-Cohen, 2017). For example, illusory shapes have been shown to increase feedback connectivity to primary visual cortex (Kok, Bains, van Mourik, Norris, \& de Lange, 2016). This raises the intriguing possibility that reductions in alpha-band feedback connectivity could underlie why ASD participants are less susceptible to visual illusions. From a predictive-coding perspective, it has been argued that top-down predictions, carried by feedback pathways, are crucial for integrating sensory features into a coherent, context-dependent percept (Palmer et al., 2017), i.e. global processing. Could findings of reduced global processing in ASD be caused by reductions in feedback connectivity and top-down predictions (Bölte, Holtmann, 
Poustka, Scheurich, \& Schmidt, 2007; Kessler et al., 2016; Robertson \& Baron-Cohen, 2017; Van Boxtel \& Lu, 2013)? These ideas could be tested through the use of experimental paradigms which modulate the top-down nature of visual processing, rather than a simple visual grating (used in my experiments), in combination with the measures of directed functional connectivity like those outlined in Chapter 3 (Bastos et al., 2015; Michalareas et al., 2016). Potential experiments are discussed further in Box 7.1.

It is also worth noting that where perception can be achieved via only feedforward processes (VanRullen \& Koch, 2003), i.e. information flowing up through the hierarchy, autistic participants will perform on par or even outperform their typically developing peers (Mottron, Dawson, Soulieres, Hubert, \& Burack, 2006). For example, autistic participants perform faster during visual search tasks (O'Riordan, Plaisted, Driver, \& Baron-Cohen, 2001), potentially because a bias toward feedforward parallel visual feature processing in ASD is likely to yield better results in this task compared to neurotypical processing, where irrelevant, distracting feedback information interferes with feedforward feature processing (Jobs, Falck-Ytter, \& Bölte, 2018; Kaldy, Giserman, Carter, \& Blaser, 2016).

\subsubsection{Integrating Oscillopathies with Predictive Coding Theories of ASD}

As argued in Chapter 1, also see Kessler et al., (2016), the study of oscillations has the potential to bridge theories of E-I in ASD at the cellular level, and neurocognitive theories of predictive-coding of ASD at the systems level (Kessler et al., 2016). Indeed, the findings of Chapter 3 integrate surprisingly well with predictive-coding theories of ASD and the results of Chapter 5 , where we tested predictive-coding theories of autism in the auditory domain. Figure 7.4 expands on these ideas in greater detail. Evidence is gathering that predictive-coding in controls is implemented through frequency-dependent hierarchical patterns of neural activity (Bastos et al., 2012; Friston, 2008). Top-down predictions flow down the cortical hierarchy (from higher-level regions to primary sensory regions), and prediction errors up the hierarchy (from primary sensory regions to higherlevel regions). Predictions, carried by feedback pathways, are associated with lowfrequency (e.g. alpha-band) oscillations, whereas prediction errors, carried by feedforward pathways, are associated with gamma oscillations (Arnal \& Giraud, 2012; Bastos et al., 2012; Bastos et al., 2015; Chao, Takaura, Wang, Fujii, \& Dehaene, 2018; Kessler et al., 2016). Based on this balance between feedforward/feedback connectivity, gamma oscillations originating from superficial layers of cortex can couple with alpha oscillations originating from deeper layers of cortex. In this way, local gamma is organised efficiently at the local level, i.e. through PAC (Bonnefond, Kastner, \& Jensen, 2017; Spaak et al., 2012). For the ASD group, predictive-coding theories suggest that neural activity is characterised by reduced top-down predictions. This would be reflected in reductions to 
feedback connectivity mediated by low-frequency (alpha-band) oscillations (see Chapter 3). The reduction in feedback connectivity would also affect the coupling between alpha/gamma oscillations at the local level, resulting in dysregulated neural activity, i.e. reduced PAC (see Chapter 3) (Berman et al., 2015; Khan et al., 2013). Future M/EEG research should test this framework in order to explicitly link oscillatory connectivity, hierarchical predictive coding and ASD symptomatology. 


\section{Box 7.1}

In chapter 3, findings of reduced feedback connectivity and reduced PAC in ASD were reported, using a static visual grating stimulus. To build on these results, future MEG research could utilise similar analysis methods (i.e. feedforward/feedback connectivity; PAC) in combination with paradigms which modulate the "top-down" nature of visual perception. I propose three potential experiments, which are discussed below.

A

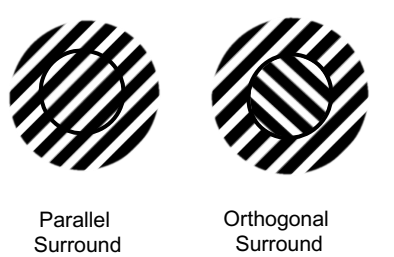

B

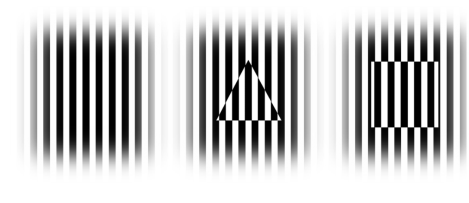

C

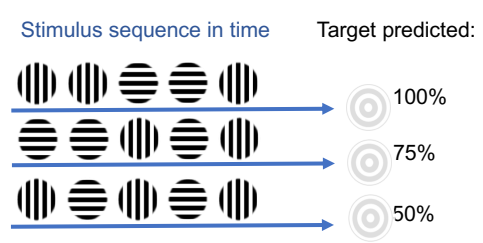

Figure 7.3 Three potential experiments to investigate oscillopathies within the visual system for ASD populations.

In order to enhance task requirements for lateral and vertical visual integration, this proposed experiment would compare visual gratings in which a central section is parallel or orthogonal to the surrounding orientation of the grating (Figure 7.3A). The typical V1 response to a visual grating is reduced for "orthogonal surround" vs "parallel surround" gratings (Zipser, Lamme, \& Schiller, 1996). This contextual modulation effect has been attributed to feedback from high-tolow visual regions (Muckli et al., 2012).

To augment the semantic and visual context of visual stimuli, one could embed visual "gestalts" (e.g. triangle or square), into basic grating stimuli by spatially phase-shifting the shape in relation to the background (Figure 7.3B). Whilst object classification in humans has been predominantly in terms of feedforward pathways (DiCarlo, Zoccolan, \& Rust, 2012), there is emerging evidence for the role of recurrent feedback processing (Wyatte, Curran \& O'Reilly, 2012). This is especially important where objects are partially occluded or degraded, and could extend to the gestalt stimuli employed here.

Converging evidence suggests that low-frequency delta and theta-band oscillations $(1-7 \mathrm{~Hz})$ from the prefrontal cortex implement context-dependent processing and anticipatory attention (Helfrich, Breska, \& Knight, 2019). For example, Helfrich, Huang, Wilson, \& Knight (2017) recently showed that predictive cues exert top-down influence over visual perception via frontal-delta / posterior-alpha PAC. In this proposed experiment, one could test the hypothesis of deficient predictive processing in autism by employing a modified version of Helfrich et al's paradigm (Figure 7.3C). Sequences of grating orientations would predict an upcoming nearthreshold target to varying degrees $(100 \%, 75 \%, 50)$. 


\section{Controls}

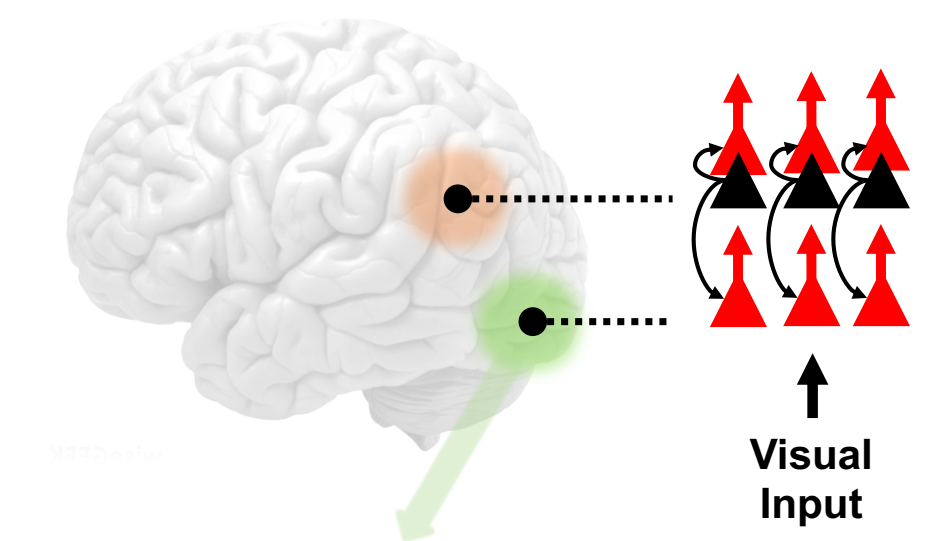

\section{ASD}

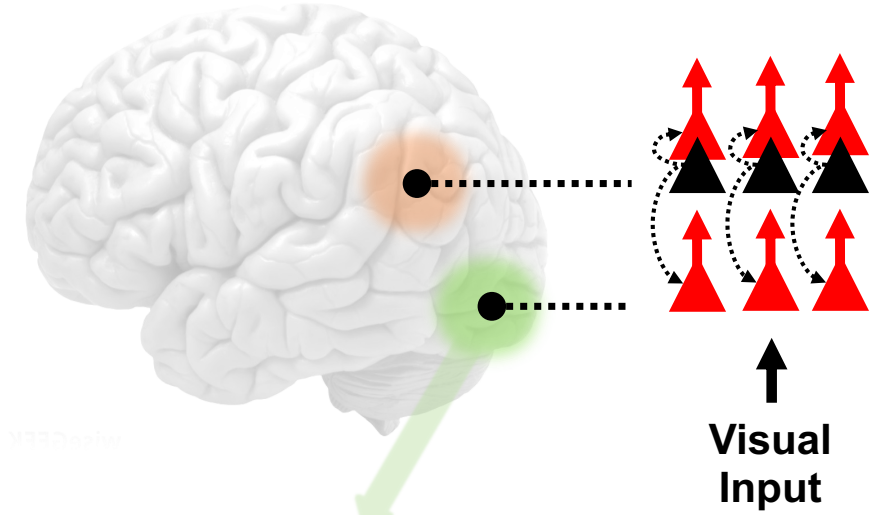

Input
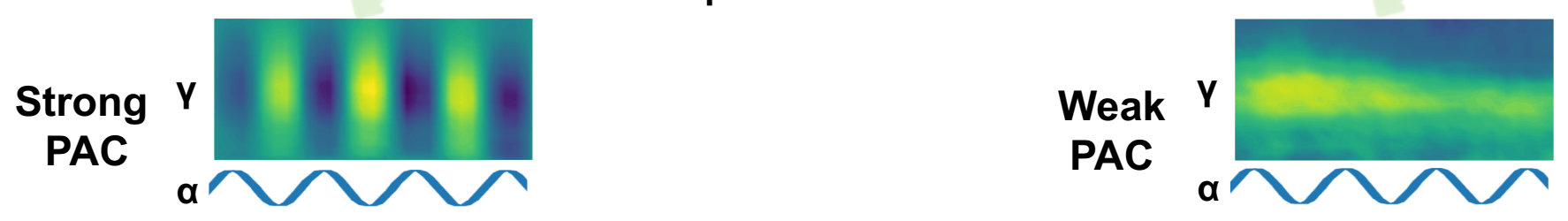

$$
\begin{gathered}
\text { Prediction error } \\
\text { (superficial } \\
\text { pyramidal cells) } \\
\text { Gamma oscillations }
\end{gathered}
$$

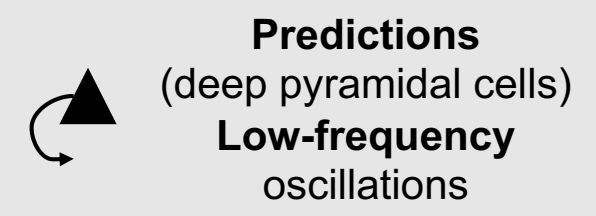
Primary
Sensory
Region

Higher-

level

Region

Figure 7.4 Proposal for how predictive-coding theories of ASD can be integrated with findings of reduced feedback connectivity and reduced PAC in ASD. Further explanations in text. 


\subsubsection{Extending the framework beyond sensory aberrations}

Whilst this thesis has primarily focussed on low-level sensory aberrations in ASD, in Chapter 1 we argued that higher-level social and cognitive impairments could also be understood through our novel framework based on neural oscillations (Kessler et al., 2016). Reductions in global phase-synchrony would especially impact higher-level cognitive processes (e.g. social cognition, perspective-taking, working memory etc.), which are dependent upon connectivity between spatially distributed cortical regions. Indeed, several studies have examined oscillatory power and connectivity in ASD, showing reductions in low-frequency power and connectivity (Bangel et al., 2014; Doesburg et al., 2013; Khan et al., 2013; Leung, Ye, Wong, Taylor, \& Doesburg, 2014; Peiker et al., 2015). However no MEG-ASD study to date has specifically investigated oscillatory power/connectivity during a task drawing on social cognition or related processes.

In Chapter 6 we therefore investigated the neural correlates of perspective-taking in control participants (Experiment 1: adults; Experiment 2: adolescents), and adolescent participants diagnosed with ASD. Perspective-taking is a high-level process which allows humans to understand how the world looks from another's point of view (Flavell, Everett, Croft, \& Flavell, 1981). Behavioural and neuroimaging research suggests that perspectivetaking depends upon embodied processes, in which people rotate their own body schema to mentally put themselves in another's perspective (Kessler \& Thomson, 2010; Surtees et al., 2013). Using MEG, we showed that for controls (adult and adolescent), increasing the angle of disparity between self and other perspectives was accompanied by increased theta power, within right lateral PFC (Experiment 1 \& 2), right TPJ (Experiment 1), right ACC (Experiment 1), ventral visual cortex (Experiment 2). Using functional connectivity analyses, we found that during perspective-taking, the rTPJ increases its theta-phase coupling with social-brain regions and regions which code for the body schema (i.e. motor cortex and supplementary motor areas). Furthermore, we showed that the lateral PFC and ACC exert top-down influence over the rTPJ. Compared with typically developing controls, ASD participants displayed reduced theta-band power during perspective-taking, as well as reductions in directed functional connectivity. Overall, these results show that lowfrequency theta-band oscillations are the crucial neural frequency for promoting connectivity between higher-level brain regions during perspective-taking (Bögels, Barr, Garrod, \& Kessler, 2015; Gooding-Williams, Wang, \& Kessler, 2017; Wang et al., 2016). Reductions in theta power and connectivity for the ASD group, therefore reflect a failure to engage higher-level brain regions, during perspective-taking. 
Interestingly, despite reductions in theta-band oscillations, increasing the angle of disparity between self and other perspectives for the ASD group was accompanied by greater alpha-band $(8-12 \mathrm{~Hz})$ desynchronisation compared with controls, within visual cortex. This decrease in power over visual regions suggests a bias towards bottom-up sensory processing in ASD (Kessler et al., 2016; Klimesch, 2012; Palmer et al., 2017), rather than top-down manipulation of internal representations supported by higher-level brain regions (e.g. rTPJ, lateral PFC, right ACC). We tentatively suggest that the different oscillatory signatures reported between ASD and control groups (i.e. theta versus alpha), could reflect differences in strategy and the underlying neurocognitive processes used to achieve perspective-taking. Unlike controls who use an embodied egocentric strategy, behavioural research has shown that children and adults with ASD are more likely to "calculate" a perspective-taking outcome (another's visuospatial viewpoint) through a mental rotation strategy, in which they rotate the world towards themselves (Pearson, Marsh, Ropar, \& Hamilton, 2016; Pearson, Ropar, \& Hamilton, 2013). The alpha desynchronisation reported over visual cortex for the ASD could reflect enhanced visual operations, required for the effortful rotation of the mental image (Chen, Bin, Daly, \& Gao, 2013). Future research could investigate this further by measuring mental rotation and perspective-taking, in terms of reaction time and brain activity, within the same participants.

Extrapolating these findings to social cognition more generally, I hypothesise that ASD would be related to reductions in connectivity between high-level brain regions, mediated by low-frequency theta-band $(3-7 \mathrm{~Hz})$ oscillations (see Figure 7.5 for more details). This would be consistent with findings from fMRI literature showing reductions in global connectivity during mentalising tasks in ASD (Gotts et al., 2012; Murdaugh et al., 2012; von dem Hagen, Stoyanova, Baron-Cohen, \& Calder, 2012). In addition, based on the results of Chapter 3 , one might expect decreased feedback connectivity from higher-level regions (e.g. the TPJ), to low-level visual cortex during social processing. This would reflect reduced top-down predictions flowing down the cortical hierarchy, consistent with predictive-coding theories of autism (Palmer et al., 2017). The results of Chapter 6 , suggest that in situations where high-level tasks can be achieved through low-level mechanisms associated with the manipulation of sensory information in a bottom-up manner (e.g. mental rotation), brain activity in ASD might be increased within sensory regions (e.g. occipital cortex). Future research should test this framework using MEG/EEG in combination with carefully designed social cognitive paradigms. 

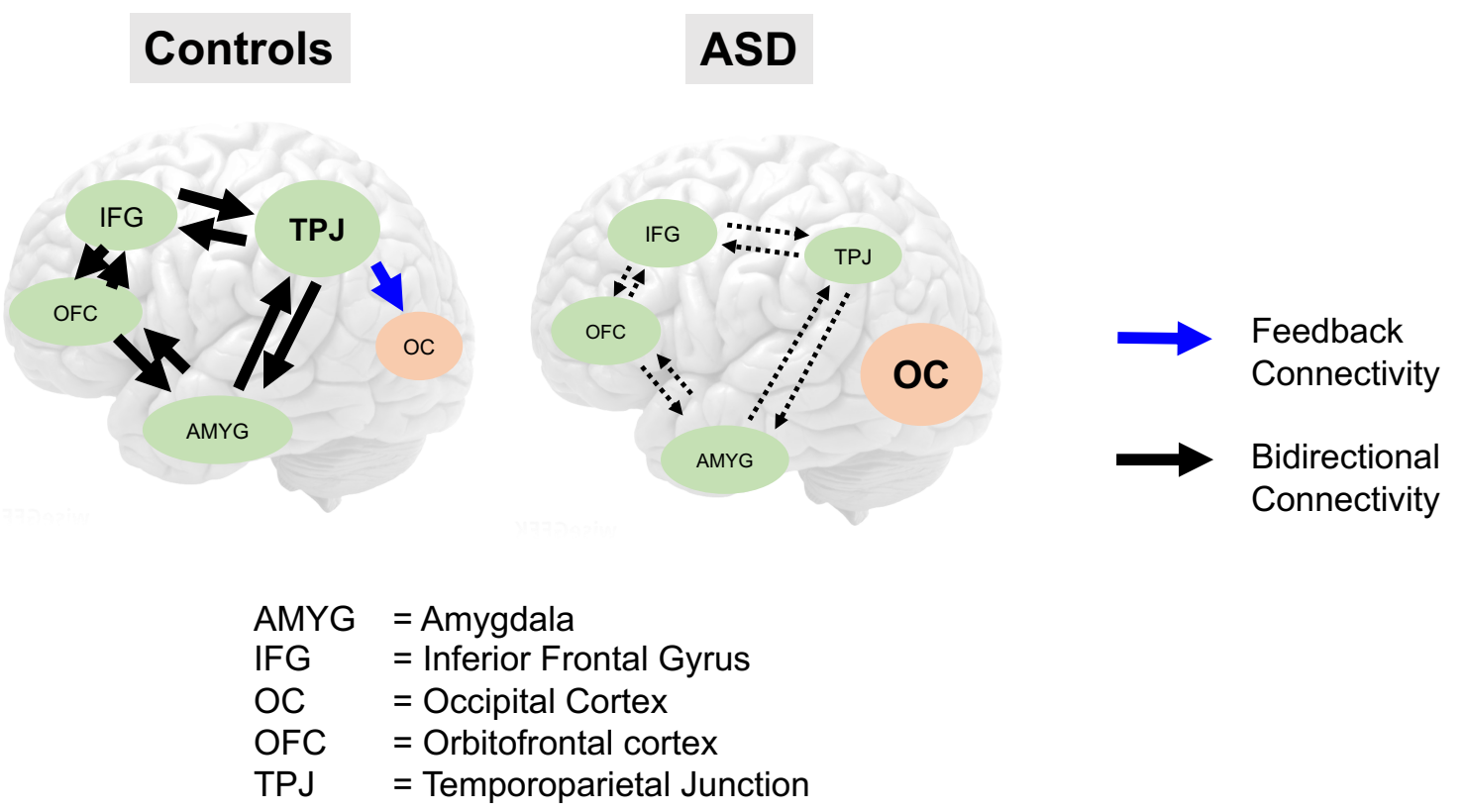

Figure 7.5 Proposed framework for the neural basis of social/cognitive symptoms of ASD. Nodes of the network have been adapted from Kessler et al., (2016) and are employed as an illustration rather than a veridical model. It is hypothesised that during social tasks/interaction, brain activity in ASD would differ from typically developing controls in 3 ways: 1) Reduced connectivity between social brain regions (mediated by low-frequency theta-band phase synchrony); 2) Reduced feedback connectivity from higher-level 'social' regions to occipital cortex; 3) Increased activity within occipital cortex for instances where social tasks can be 'solved' using a bottom-up strategy. Further explanations in text.

\subsubsection{Heterogeneity in ASD}

Whilst this thesis has primarily focussed on the heterogeneity of brain activity between ASD participants compared with controls, it should be noted that there is considerable heterogeneity within ASD cohorts in terms of symptomatology, cognitive profiles and comorbidities such as epilepsy, schizophrenia and intellectual disability (Jeste \& Geschwind, 2014; Szatmari, 1999). It has even been argued that the singular clinical condition "ASD" should be reframed as "the autisms" (Jeste \& Geschwind, 2014). In terms of neuroimaging, behavioural heterogeneity may also result in the heterogenous and sometimes counterintuitive findings reported in relation to functional connectivity, also see Gicci et al., (2016). Future neuroimaging studies should therefore attempt to collect detailed clinical information from ASD cohorts and potentially cluster the data into independent subgroups (Lenroot \& Yeung, 2013). However, this will obviously require large ASD sample sizes and appropriate statistical tools to identify genuine subgroups defined by genetics and/or symptom profiles. 
It should be noted that one advantage of our framework (see Chapter 1 ) is that it does not concentrate on one particular ASD symptom, but rather focusses on establishing which underlying neurobiological pathways might be affected (Geschwind \& Levitt, 2007). If ASD is related to local dysregulation and global hypo-connectivity, as argued in this thesis, then the resulting "symptoms" would depend on the specific developmental stage and neurocognitive pathways affected (Kessler et al., 2016). Rather than being something research should try to address, behavioural and cognitive heterogeneity should be embraced as an important aspect of ASD (Geschwind \& Levitt, 2007).

One particularly exciting research area related to this heterogeneity is that of "idiosyncratic" brain activity. Using fMRI, Hahamy, Behrmann \& Malach (2016) showed that the spatial pattern of interhemispheric connectivity was more variable (or "idiosyncratic") for a group of ASD participants compared with controls. In other words, ASD participants differed more from the group average spatial map of interhemispheric connectivity, than controls. Similar findings were also reported by Byrge et al., (2015) and more recently by Nunes et al., (2018) who showed that the somatosensory and default mode networks were more spatially variable in autism. In future, similar analysis approaches could be applied to M/EEG metrics of functional connectivity to establish whether oscillatory activity mirrors the behavioural heterogeneity of ASD.

\subsubsection{Implications for ASD Symptomatology}

More generally, the results of Chapters 3-5, show that neural activity in ASD is characterised by differences in the cortical mechanisms underlying low-level sensory perception. This is important, given the increasing interest in characterising the sensory symptoms of ASD (Kessler et al., 2016; Robertson \& Baron-Cohen, 2017; Rojas \& Wilson, 2014; Simon \& Wallace, 2016). Sensory aberrations can significantly impact the quality of life for autistic children and adults, sometimes to a greater extent than the higher-level and social aspects of ASD (Leekam, Nieto, Libby, Wing, \& Gould, 2007). From a clinical perspective, the findings of this thesis could inform translational research aimed at alleviating sensory hyper/hypo-sensitives and atypical developmental trajectories in social cognition (Kessler et al., 2016). For example, neurofeedback training has been shown to be able to modify the power of gamma oscillations during sensory processing (Keizer, Verschoor, Verment, \& Hommel, 2010). Similar neurofeedback training, in combination with carefully targeted interventions, could potentially help to modify findings of reduced feedback connectivity and PAC in ASD (Simon \& Wallace, 2016). Additionally, with the advent of techniques such as transcranial alternating current stimulation, it may be possible to physically modify cortical oscillations in ASD patients (Witkowski et al., 2016). 


\subsection{Conclusion}

In conclusion, this thesis has utilised the spatiotemporal properties of MEG, in combination with robust analysis techniques, to investigate the neural basis of ASD. Data were collected from adolescents diagnosed with ASD and age-matched controls, at Aston University and Macquarie University. Based on previous research, it was hypothesised that cortical activity in ASD participants would be associated with disruptions to oscillatory synchronisation during early sensory processing, as well as during high-level perspectivetaking. More specifically, a novel framework for the study of oscillopathies in ASD was introduced in Chapter 1, based on local gamma-band dysregulation, global hypoconnectivity and deficient predictive-coding, also see Kessler et al., (2016). Our experimental findings of Chapter 3-6 largely support this framework. In the visual domain, it was found that ASD participants had reduced PAC as well as reduced feedback connectivity (mediated by alpha-band oscillations). In the auditory domain, it was found that ASD participants had reduced steady-state responses at $40 \mathrm{~Hz}$, in terms of oscillatory power and ITC. Investigating predictive-coding theories of ASD using an auditory oddball paradigm, it was found that evoked responses to the omission of an expected tone were reduced for the ASD group. Finally, we found reductions in theta-band oscillatory power and connectivity for ASD participants, during higher-level perspective-taking. Future research should build on the findings presented in this thesis, using MEG in combination with larger and more diverse autistic samples, to determine how oscillopathies link with ASD symptomatology. 


\section{References}

Alcalá-López, D., Smallwood, J., Jefferies, E., Van Overwalle, F., Vogeley, K., Mars, R. B., ... Eickhoff, S. B. (2017). Computing the social brain connectome across systems and states. Cerebral Cortex, 28(7), 2207-2232.

Amodio, D. M., \& Frith, C. D. (2006). Meeting of minds: the medial frontal cortex and social cognition. Nature Reviews. Neuroscience, 7(4), 268.

APA, -. (2013). Diagnostic and statistical manual of mental disorders (DSM-5®). American Psychiatric Pub.

Arnal, L. H., \& Giraud, A.-L. (2012). Cortical oscillations and sensory predictions. Trends in Cognitive Sciences, 16(7), 390-398.

Aron, A. R., Robbins, T. W., \& Poldrack, R. A. (2014). Inhibition and the right inferior frontal cortex: one decade on. Trends in Cognitive Sciences, 18(4), 177-185.

Aru, J., Aru, J., Priesemann, V., Wibral, M., Lana, L., Pipa, G., ... Vicente, R. (2015). Untangling crossfrequency coupling in neuroscience. Current Opinion in Neurobiology, 31, 51-61.

Arzy, S., Thut, G., Mohr, C., Michel, C. M., \& Blanke, O. (2006). Neural Basis of Embodiment: Distinct Contributions of Temporoparietal Junction and Extrastriate Body Area. Journal of Neuroscience, 26(31), 8074-8081.

Ashwin, E., Ashwin, C., Rhydderch, D., Howells, J., \& Baron-Cohen, S. (2009). Eagle-eyed visual acuity: an experimental investigation of enhanced perception in autism. Biological Psychiatry, 65(1), 17-21.

Auksztulewicz, R., Friston, K. J., \& Nobre, A. C. (2017). Task relevance modulates the behavioural and neural effects of sensory predictions. PLOS Biology, 15(12), e2003143.

Azzena, G. B., Conti, G., Santarelli, R., Ottaviani, F., Paludetti, G., \& Maurizi, M. (1995). Generation of human auditory steadystate responses (SSRs). I: Stimulus rate effects. Hearing Research, 83(12), $1-8$.

Bangel, K. A., Batty, M., Ye, A. X., Meaux, E., Taylor, M. J., \& Doesburg, S. M. (2014). Reduced beta band connectivity during number estimation in autism. Neurolmage: Clinical, 6, 202-213.

Barascud, N., Pearce, M. T., Griffiths, T. D., Friston, K. J., \& Chait, M. (2016). Brain responses in humans reveal ideal observer-like sensitivity to complex acoustic patterns. Proceedings of the National Academy of Sciences, 113(5), E616-E625.

Baron-Cohen, S., Ashwin, E., Ashwin, C., Tavassoli, T., \& Chakrabarti, B. (2009). Talent in autism: hypersystemizing, hyper-attention to detail and sensory hypersensitivity. Philosophical Transactions of the Royal Society of London B: Biological Sciences, 364(1522), 1377-1383.

Baron-Cohen, S., Scott, F. J., Allison, C., Williams, J., Bolton, P., Matthews, F. E., \& Brayne, C. (2009). Prevalence of autism-spectrum conditions: UK school-based population study. The British Journal of Psychiatry, 194(6), 500-509.

Baron-Cohen, S., Wheelwright, S., Hill, J., Raste, Y., \& Plumb, I. (2001). The "Reading the Mind in the Eyes" test revised version: A study with normal adults, and adults with Asperger syndrome or highfunctioning autism. Journal of Child Psychology and Psychiatry, 42(2), 241-251.

Barone, P., Batardiere, A., Knoblauch, K., \& Kennedy, H. (2000). Laminar distribution of neurons in extrastriate areas projecting to visual areas V1 and V4 correlates with the hierarchical rank and indicates the operation of a distance rule. Journal of Neuroscience, 20(9), 3263-3281.

Barratt, E. L., Francis, S. T., Morris, P. G., \& Brookes, M. J. (2018). Mapping the topological organisation of beta oscillations in motor cortex using MEG. Neurolmage.

Barttfeld, P., Wicker, B., Cukier, S., Navarta, S., Lew, S., Leiguarda, R., \& Sigman, M. (2012). Statedependent changes of connectivity patterns and functional brain network topology in autism spectrum disorder. Neuropsychologia, 50(14), 3653-3662.

Bastiaansen, M. C., \& Knösche, T. R. (2000). Tangential derivative mapping of axial MEG applied to eventrelated desynchronization research. Clinical Neurophysiology, 111(7), 1300-1305.

Bastos, André M., Litvak, V., Moran, R., Bosman, C. A., Fries, P., \& Friston, K. J. (2015). A DCM study of spectral asymmetries in feedforward and feedback connections between visual areas V1 and V4 in the monkey. Neuroimage, 108, 460-475.

Bastos, André M., \& Schoffelen, J.-M. (2016). A Tutorial Review of Functional Connectivity Analysis Methods and Their Interpretational Pitfalls. Frontiers in Systems Neuroscience, 175.

Bastos, Andre M., Usrey, W. M., Adams, R. A., Mangun, G. R., Fries, P., \& Friston, K. J. (2012). Canonical microcircuits for predictive coding. Neuron, 76(4), 695-711. 
Bastos, Vezoli, J., Bosman, C. A., Schoffelen, J.-M., Oostenveld, R., Dowdall, J. R., ... Fries, P. (2015). Visual Areas Exert Feedforward and Feedback Influences through Distinct Frequency Channels. Neuron, 85(2), 390-401.

Baum, S. H., Stevenson, R. A., \& Wallace, M. T. (2015). Behavioral, perceptual, and neural alterations in sensory and multisensory function in autism spectrum disorder. Progress in Neurobiology, 134, 140-160.

Belmonte, M. K., Allen, G., Beckel-Mitchener, A., Boulanger, L. M., Carper, R. A., \& Webb, S. J. (2004). Autism and abnormal development of brain connectivity. The Journal of Neuroscience, 24(42), 9228-9231.

Bendixen, A., SanMiguel, I., \& Schröger, E. (2012). Early electrophysiological indicators for predictive processing in audition: A review. International Journal of Psychophysiology, 83(2), 120-131.

Benjamini, Y., \& Hochberg, Y. (1995). Controlling the false discovery rate: a practical and powerful approach to multiple testing. Journal of the Royal Statistical Society. Series B (Methodological), 289-300.

Berg, A. T., \& Plioplys, S. (2012). Epilepsy and autism: is there a special relationship? Epilepsy \& Behavior, 23(3), 193-198.

Berman, J. I., Liu, S., Bloy, L., Blaskey, L., Roberts, T. P., \& Edgar, J. C. (2015). Alpha-to-gamma phaseamplitude coupling methods and application to autism spectrum disorder. Brain Connectivity, 5(2), 80-90.

Berman, J. I., McDaniel, J., Liu, S., Cornew, L., Gaetz, W., Roberts, T. P., \& Edgar, J. C. (2012). Variable bandwidth filtering for improved sensitivity of cross-frequency coupling metrics. Brain Connectivity, 2(3), 155-163.

Blakemore, S.-J. (2012). Development of the social brain in adolescence. Journal of the Royal Society of Medicine, 105(3), 111-116.

Blanke, O., Mohr, C., Michel, C. M., Pascual-Leone, A., Brugger, P., Seeck, M., ... Thut, G. (2005). Linking out-of-body experience and self processing to mental own-body imagery at the temporoparietal junction. Journal of Neuroscience, 25(3), 550-557.

Bogdashina, O. (2003). Sensory perceptual issues in autism and Asperger syndrome: Different sensory experiences--different perceptual worlds. London, England: Jessica Kingsley Publishers.

Bögels, S., Barr, D. J., Garrod, S., \& Kessler, K. (2015). Conversational interaction in the scanner: mentalizing during language processing as revealed by MEG. Cerebral Cortex, 25(9), 3219-3234.

Bölte, S., Holtmann, M., Poustka, F., Scheurich, A., \& Schmidt, L. (2007). Gestalt perception and localglobal processing in high-functioning autism. Journal of Autism and Developmental Disorders, 37(8), 1493-1504.

Bonnefond, M., \& Jensen, O. (2015). Gamma Activity Coupled to Alpha Phase as a Mechanism for TopDown Controlled Gating. PloS One, 10(6), e0128667.

Bonnefond, M., Kastner, S., \& Jensen, O. (2017). Communication between Brain Areas Based on Nested Oscillations. ENeuro, 4(2), ENEURO-0153.

Bosman, C. A., Lansink, C. S., \& Pennartz, C. (2014). Functions of gamma-band synchronization in cognition: from single circuits to functional diversity across cortical and subcortical systems. European Journal of Neuroscience, 39(11), 1982-1999.

Botvinick, M. M., Cohen, J. D., \& Carter, C. S. (2004). Conflict monitoring and anterior cingulate cortex: an update. Trends in Cognitive Sciences, 8(12), 539-546.

Bradford, E. E. F., Jentzsch, I., \& Gomez, J.-C. (2015). From self to social cognition: Theory of Mind mechanisms and their relation to Executive Functioning. Cognition, 138, 21-34.

Bragin, A., Jandó, G., Nádasdy, Z., Hetke, J., Wise, K., \& Buzsáki, G. (1995). Gamma (40-100 Hz) oscillation in the hippocampus of the behaving rat. Journal of Neuroscience, 15(1), 47-60.

Bräuer, J., Call, J., \& Tomasello, M. (2007). Chimpanzees really know what others can see in a competitive situation. Animal Cognition, 10(4), 439-448.

Brock, J., Brown, C. C., Boucher, J., \& Rippon, G. (2002). The temporal binding deficit hypothesis of autism. Development and Psychopathology, 14(02), 209-224.

Brookes, M. J., Hale, J. R., Zumer, J. M., Stevenson, C. M., Francis, S. T., Barnes, G. R., ... Nagarajan, S. S. (2011). Measuring functional connectivity using MEG: methodology and comparison with fcMRI. Neuroimage, 56(3), 1082-1104.

Brown, C., Gruber, T., Boucher, J., Rippon, G., \& Brock, J. (2005). Gamma abnormalities during perception of illusory figures in autism. Cortex, 41(3), 364-376. 
Bugnyar, T., Stöwe, M., \& Heinrich, B. (2004). Ravens, Corvus corax, follow gaze direction of humans around obstacles. Proceedings of the Royal Society of London B: Biological Sciences, 271(1546), 1331-1336.

Busch, N. A., Dubois, J., \& VanRullen, R. (2009). The phase of ongoing EEG oscillations predicts visual perception. Journal of Neuroscience, 29(24), 7869-7876.

Buzsáki, G., \& Draguhn, A. (2004). Neuronal oscillations in cortical networks. Science, 304(5679), 19261929.

Buzsáki, G., \& Wang, X.-J. (2012). Mechanisms of gamma oscillations. Annual Review of Neuroscience, 35, 203.

Bzdok, D., Langner, R., Schilbach, L., Jakobs, O., Roski, C., Caspers, S., ... Eickhoff, S. B. (2013). Characterization of the temporo-parietal junction by combining data-driven parcellation, complementary connectivity analyses, and functional decoding. Neurolmage, 81, 381-392.

Canolty, R. T., Edwards, E., Dalal, S. S., Soltani, M., Nagarajan, S. S., Kirsch, H. E., ... Knight, R. T. (2006). High gamma power is phase-locked to theta oscillations in human neocortex. Science, 313(5793), 1626-1628.

Canolty, R. T., \& Knight, R. T. (2010). The functional role of cross-frequency coupling. Trends in Cognitive Sciences, 14(11), 506-515.

Cao, W., Lin, S., Xia, Q., Du, Y., Yang, Q., Zhang, M., ... Luo, J. (2018). Gamma Oscillation Dysfunction in mPFC Leads to Social Deficits in Neuroligin 3 R451C Knockin Mice. Neuron, 97(6), 1253-1260.e7.

Capotosto, P., Babiloni, C., Romani, G. L., \& Corbetta, M. (2009). Frontoparietal cortex controls spatial attention through modulation of anticipatory alpha rhythms. Journal of Neuroscience, 29(18), 58635872.

Cardy, J. E. O., Flagg, E. J., Roberts, W., \& Roberts, T. P. (2005). Delayed mismatch field for speech and non-speech sounds in children with autism. Neuroreport, 16(5), 521-525.

Carrington, S. J., \& Bailey, A. J. (2009). Are there theory of mind regions in the brain? A review of the neuroimaging literature. Human Brain Mapping, 30(8), 2313-2335.

Carter, R. M., \& Huettel, S. A. (2013). A nexus model of the temporal-parietal junction. Trends in Cognitive Sciences, 17(7), 328-336.

Casanova, M. F., van Kooten, I., Switala, A. E., van Engeland, H., Heinsen, H., Steinbusch, H. W. M., ... Schmitz, C. (2006). Abnormalities of cortical minicolumnar organization in the prefrontal lobes of autistic patients. Clinical Neuroscience Research, 6(3-4), 127-133.

Casanova, Manuel F., Buxhoeveden, D., \& Gomez, J. (2003). Disruption in the inhibitory architecture of the cell minicolumn: implications for autisim. The Neuroscientist, 9(6), 496-507.

Casanova, Manuel F., Buxhoeveden, D. P., \& Brown, C. (2002). Clinical and macroscopic correlates of minicolumnar pathology in autism. Journal of Child Neurology, 17(9), 692-695.

Cavanagh, J. F., \& Frank, M. J. (2014). Frontal theta as a mechanism for cognitive control. Trends in Cognitive Sciences, 18(8), 414-421.

Cazzato, V., Mian, E., Serino, A., Mele, S., \& Urgesi, C. (2015). Distinct contributions of extrastriate body area and temporoparietal junction in perceiving one's own and others' body. Cognitive, Affective, \& Behavioral Neuroscience, 15(1), 211-228.

Chacko, R. V., Kim, B., Jung, S. W., Daitch, A. L., Roland, J. L., Metcalf, N. V., ... Leuthardt, E. C. (2018). Distinct phase-amplitude couplings distinguish cognitive processes in human attention. Neurolmage, 175, 111-121.

Chao, Z. C., Takaura, K., Wang, L., Fujii, N., \& Dehaene, S. (2018a). Large-Scale Cortical Networks for Hierarchical Prediction and Prediction Error in the Primate Brain. Neuron, 100(5), 1252-1266.

Chao, Z. C., Takaura, K., Wang, L., Fujii, N., \& Dehaene, S. (2018b). Large-Scale Cortical Networks for Hierarchical Prediction and Prediction Error in the Primate Brain. Neuron, 100(5), 1252-1266.e3.

Chen, X., Bin, G., Daly, I., \& Gao, X. (2013). Event-related desynchronization (ERD) in the alpha band during a hand mental rotation task. Neuroscience Letters, 541, 238-242.

Cheng, N., Li, Q., Wang, S., Wang, R., \& Zhang, T. (2018). Permutation Mutual Information: A Novel Approach for Measuring Neuronal Phase-Amplitude Coupling. Brain Topography, 31(2), 186-201.

Chennu, S., Noreika, V., Gueorguiev, D., Shtyrov, Y., Bekinschtein, T. A., \& Henson, R. (2016). Silent expectations: dynamic Causal modeling of cortical prediction and attention to sounds that Weren't. Journal of Neuroscience, 36(32), 8305-8316.

Cho, R. Y., Walker, C. P., Polizzotto, N. R., Wozny, T. A., Fissell, C., Chen, C.-M. A., \& Lewis, D. A. (2015). Development of sensory gamma oscillations and cross-frequency coupling from childhood to early adulthood. Cerebral Cortex, 25(6), 1509-1518. 
Coghlan, S., Horder, J., Inkster, B., Mendez, M. A., Murphy, D. G., \& Nutt, D. J. (2012). GABA system dysfunction in autism and related disorders: from synapse to symptoms. Neuroscience \& Biobehavioral Reviews, 36(9), 2044-2055.

Cohen, M. X. (2008). Assessing transient cross-frequency coupling in EEG data. Journal of Neuroscience Methods, 168(2), 494-499.

Colclough, G. L., Brookes, M. J., Smith, S. M., \& Woolrich, M. W. (2015). A symmetric multivariate leakage correction for MEG connectomes. Neurolmage, 117, 439-448.

Cole, S. R., van der Meij, R., Peterson, E. J., de Hemptinne, C., Starr, P. A., \& Voytek, B. (2017). Nonsinusoidal beta oscillations reflect cortical pathophysiology in Parkinson's disease. Journal of Neuroscience, 37(18), 4830-4840.

Cole, S. R., \& Voytek, B. (2017). Brain Oscillations and the Importance of Waveform Shape. Trends in Cognitive Sciences, 21(2), 137-149.

Conson, M., Mazzarella, E., Esposito, D., Grossi, D., Marino, N., Massagli, A., \& Frolli, A. (2015). "Put Myself Into Your Place": Embodied Simulation and Perspective Taking in Autism Spectrum Disorders. Autism Research, 8(4), 454-466.

Cornew, L., Roberts, T. P., Blaskey, L., \& Edgar, J. C. (2012). Resting-state oscillatory activity in autism spectrum disorders. Journal of Autism and Developmental Disorders, 42(9), 1884-1894.

Coslett, H., Buxbaum, L. J., \& Schwoebel, J. (2008). Accurate reaching after active but not passive movements of the hand: Evidence for forward modeling. Behavioural Neurology, 19(3), 117-125.

Courchesne, E., \& Pierce, K. (2005). Why the frontal cortex in autism might be talking only to itself: local over-connectivity but long-distance disconnection. Current Opinion in Neurobiology, 15(2), 225230.

d'Albis, M.-A., Guevara, P., Guevara, M., Laidi, C., Boisgontier, J., Sarrazin, S., ... Czech, C. (2018). Local structural connectivity is associated with social cognition in autism spectrum disorder. Brain, 141(12), 3472-3481.

Dajani, D. R., \& Uddin, L. Q. (2016). Local brain connectivity across development in autism spectrum disorder: A cross-sectional investigation. Autism Research, 9(1), 43-54.

Dakin, S., \& Frith, U. (2005). Vagaries of Visual Perception in Autism. Neuron, 48(3), 497-507.

David, O., Kilner, J. M., \& Friston, K. J. (2006). Mechanisms of evoked and induced responses in MEG/EEG. Neuroimage, 31(4), 1580-1591.

De Hemptinne, C., Ryapolova-Webb, E. S., Air, E. L., Garcia, P. A., Miller, K. J., Ojemann, J. G., ... Starr, P. A. (2013). Exaggerated phase-amplitude coupling in the primary motor cortex in Parkinson disease. Proceedings of the National Academy of Sciences, 110(12), 4780-4785.

Dhamala, M., Rangarajan, G., \& Ding, M. (2008). Analyzing information flow in brain networks with nonparametric Granger causality. Neuroimage, 41(2), 354-362.

Di Russo, F., Martínez, A., Sereno, M. I., Pitzalis, S., \& Hillyard, S. A. (2002). Cortical sources of the early components of the visual evoked potential. Human Brain Mapping, 15(2), 95-111.

Dickinson, A., Jones, M., \& Milne, E. (2016). Measuring neural excitation and inhibition in autism: Different approaches, different findings and different interpretations. Brain Research, 1648, 277-289.

Ding Mingzhou, Chen Yonghong, \& Bressler Steven L. (2006). Granger Causality: Basic Theory and Application to Neuroscience. Handbook of Time Series Analysis.

Dinstein, I., Pierce, K., Eyler, L., Solso, S., Malach, R., Behrmann, M., \& Courchesne, E. (2011). Disrupted neural synchronization in toddlers with autism. Neuron, 70(6), 1218-1225.

Doesburg, S. M., Vidal, J., \& Taylor, M. J. (2013). Reduced Theta Connectivity during Set-Shifting in Children with Autism. Frontiers in Human Neuroscience, 7.

Dreschler, W. A., Verschuure, H., Ludvigsen, C., \& Westermann, S. (2001). ICRA noises: artificial noise signals with speech-like spectral and temporal properties for hearing instrument assessment: Ruidos ICRA: Señates de ruido artificial con espectro similar al habla y propiedades temporales para pruebas de instrumentos auditivos. Audiology, 40(3), 148-157.

Duncan, J., \& Owen, A. M. (2000). Common regions of the human frontal lobe recruited by diverse cognitive demands. Trends in Neurosciences, 23(10), 475-483.

Dunn, M. A., Gomes, H., \& Gravel, J. (2008). Mismatch negativity in children with autism and typical development. Journal of Autism and Developmental Disorders, 38(1), 52-71.

Dvorak, D., \& Fenton, A. A. (2014). Toward a proper estimation of phase-amplitude coupling in neural oscillations. Journal of Neuroscience Methods, 225, 42-56.

Eddy, C. M. (2016). The junction between self and other? Temporo-parietal dysfunction in neuropsychiatry. Neuropsychologia, 89, 465-477. 
Edgar, J. C., Fisk, C. L., Liu, S., Pandey, J., Herrington, J. D., Schultz, R. T., \& Roberts, T. P. L. (2016). Translating Adult Electrophysiology Findings to Younger Patient Populations: Difficulty Measuring 40-Hz Auditory Steady-State Responses in Typically Developing Children and Children with Autism Spectrum Disorder. Developmental Neuroscience.

Edgar, J. C., Khan, S. Y., Blaskey, L., Chow, V. Y., Rey, M., Gaetz, W., ... others. (2015). Neuromagnetic oscillations predict evoked-response latency delays and core language deficits in autism spectrum disorders. Journal of Autism and Developmental Disorders, 45(2), 395-405.

Engel, A. K., \& Fries, P. (2010). Beta-band oscillations-signalling the status quo? Current Opinion in Neurobiology, 20(2), 156-165.

Engel, A. K., Fries, P., \& Singer, W. (2001). Dynamic predictions: Oscillations and synchrony in top-down processing. Nature Reviews Neuroscience, 2(10), 704-716.

Essen, V., C, D., Glasser, M. F., Dierker, D. L., Harwell, J., \& Coalson, T. (2012). Parcellations and Hemispheric Asymmetries of Human Cerebral Cortex Analyzed on Surface-Based Atlases. Cerebral Cortex, 22(10), 2241-2262.

Farley, B. J., Quirk, M. C., Doherty, J. J., \& Christian, E. P. (2010). Stimulus-specific adaptation in auditory cortex is an NMDA-independent process distinct from the sensory novelty encoded by the mismatch negativity. Journal of Neuroscience, 30(49), 16475-16484.

Felleman, D. J., \& Van, D. E. (1991). Distributed hierarchical processing in the primate cerebral cortex. Cerebral Cortex (New York, NY: 1991), 1(1), 1-47.

Fischl, B. (2012). FreeSurfer. Neuroimage, 62(2), 774-781.

Flavell, J. H., Everett, B. A., Croft, K., \& Flavell, E. R. (1981). Young children's knowledge about visual perception: Further evidence for the Level 1-Level 2 distinction. Developmental Psychology, 17(1), 99.

Florin, E., \& Baillet, S. (2015). The brain's resting-state activity is shaped by synchronized cross-frequency coupling of neural oscillations. Neurolmage, 111, 26-35.

Fontanini, A., \& Katz, D. B. (2005). 7 to $12 \mathrm{~Hz}$ activity in rat gustatory cortex reflects disengagement from a fluid self-administration task. Journal of Neurophysiology, 93(5), 2832-2840.

Freyberg, J., Robertson, C., \& Baron-Cohen, S. (2015). Atypical Binocular Rivalry Dynamics of Simple and Complex Stimuli in Autism. Journal of Vision, 15(12), 643-643.

Fries, P. (2015). Rhythms for cognition: communication through coherence. Neuron, 88(1), 220-235.

Friston, K. (2005). A theory of cortical responses. Philosophical Transactions of the Royal Society of London. Series B, Biological Sciences, 360(1456), 815-836.

Friston, K. (2008). Hierarchical models in the brain. PLoS Computational Biology, 4(11), e1000211.

Friston, K. J. (2011). Functional and effective connectivity: a review. Brain Connectivity, 1(1), 13-36.

Friston, K. J. (2017). Precision Psychiatry. Biological Psychiatry: Cognitive Neuroscience and Neuroimaging, 2(8), 640-643.

Friston, K. J., Bastos, A. M., Pinotsis, D., \& Litvak, V. (2015). LFP and oscillations-what do they tell us? Current Opinion in Neurobiology, 31, 1-6.

Friston, K. J., Lawson, R., \& Frith, C. D. (2013). On hyperpriors and hypopriors: comment on Pellicano and Burr. Trends Cogn. Sci, 17(1), 10-1016.

Fuhrmann, D., Knoll, L. J., \& Blakemore, S.-J. (2015). Adolescence as a sensitive period of brain development. Trends in Cognitive Sciences, 19(10), 558-566.

Gandal, M. J., Edgar, J. C., Ehrlichman, R. S., Mehta, M., Roberts, T. P., \& Siegel, S. J. (2010). Validating Y oscillations and delayed auditory responses as translational biomarkers of autism. Biological Psychiatry, 68(12), 1100-1106.

Gao, W.-J., Newman, D. E., Wormington, A. B., \& Pallas, S. L. (1999). Development of inhibitory circuitry in visual and auditory cortex of postnatal ferrets: immunocytochemical localization of GABAergic neurons. Journal of Comparative Neurology, 409(2), 261-273.

Garrido, M. I., Friston, K. J., Kiebel, S. J., Stephan, K. E., Baldeweg, T., \& Kilner, J. M. (2008). The functional anatomy of the MMN: a DCM study of the roving paradigm. Neuroimage, 42(2), 936944.

Garrido, M. I., Kilner, J. M., Stephan, K. E., \& Friston, K. J. (2009). The mismatch negativity: a review of underlying mechanisms. Clinical Neurophysiology, 120(3), 453-463.

Geng, J. J., \& Vossel, S. (2013). Re-evaluating the role of TPJ in attentional control: contextual updating? Neuroscience \& Biobehavioral Reviews, 37(10), 2608-2620.

Geschwind, D. H., \& Levitt, P. (2007). Autism spectrum disorders: developmental disconnection syndromes. Current Opinion in Neurobiology, 17(1), 103-111. 
Giraud, A.-L., \& Arnal, L. H. (2018). Hierarchical Predictive Information Is Channeled by Asymmetric Oscillatory Activity. Neuron, 100(5), 1022-1024.

Glasser, M. F., Coalson, T. S., Robinson, E. C., Hacker, C. D., Harwell, J., Yacoub, E., ... Van Essen, D. C. (2016). A multi-modal parcellation of human cerebral cortex. Nature, 536(7615), 171-178.

Gohel, B., Lim, S., Kim, M.-Y., Kwon, H., \& Kim, K. (2017). Approximate Subject Specific Pseudo MRI from an Available MRI Dataset for MEG Source Imaging. Frontiers in Neuroinformatics, 11, 50.

Goldenholz, D. M., Ahlfors, S. P., Hämäläinen, M. S., Sharon, D., Ishitobi, M., Vaina, L. M., \& Stufflebeam, S. M. (2009). Mapping the signal-to-noise-ratios of cortical sources in magnetoencephalography and electroencephalography. Human Brain Mapping, 30(4), 1077-1086.

Gooding-Williams, G., Wang, H., \& Kessler, K. (2017). THETA-Rhythm Makes the World Go Round: Dissociative Effects of TMS Theta Versus Alpha Entrainment of Right pTPJ on Embodied Perspective Transformations. Brain Topography, 1-4.

Goris, J., Braem, S., Nijhof, A. D., Rigoni, D., Deschrijver, E., Van de Cruys, S., ... Brass, M. (2018a). Sensory prediction errors are less modulated by global context in autism spectrum disorder. Biological Psychiatry: Cognitive Neuroscience and Neuroimaging.

Goris, J., Braem, S., Nijhof, A. D., Rigoni, D., Deschrijver, E., Van de Cruys, S., ... Brass, M. (2018b). Sensory prediction errors are less modulated by global context in autism spectrum disorder. Biological Psychiatry: Cognitive Neuroscience and Neuroimaging.

Gotts, S. J., Simmons, W. K., Milbury, L. A., Wallace, G. L., Cox, R. W., \& Martin, A. (2012). Fractionation of social brain circuits in autism spectrum disorders. Brain, 135(9), 2711-2725.

Gramfort, A., Luessi, M., Larson, E., Engemann, D. A., Strohmeier, D., Brodbeck, C., ... Hämäläinen, M. S. (2014). MNE software for processing MEG and EEG data. Neuroimage, 86, 446-460.

Granger, C. W. (1969). Investigating causal relations by econometric models and cross-spectral methods. Econometrica: Journal of the Econometric Society, 424-438.

Grice, S. J., Spratling, M. W., Karmiloff-Smith, A., Halit, H., Csibra, G., de Haan, M., \& Johnson, M. H. (2001). Disordered visual processing and oscillatory brain activity in autism and Williams syndrome. Neuroreport, 12(12), 2697-2700.

Grols s, J., Kujala, J., Hämäläinen, M., Timmermann, L., Schnitzler, A., \& Salmelin, R. (2001). Dynamic imaging of coherent sources: studying neural interactions in the human brain. Proceedings of the National Academy of Sciences, 98(2), 694-699.

Gzesh, S. M., \& Surber, C. F. (1985). Visual perspective-taking skills in children. Child Development, 12041213.

Hahamy, A., Behrmann, M., \& Malach, R. (2015). The idiosyncratic brain: distortion of spontaneous connectivity patterns in autism spectrum disorder. Nature Neuroscience, 18(2), 302-309.

Hamilton, A. F. de C., Brindley, R., \& Frith, U. (2009). Visual perspective taking impairment in children with autistic spectrum disorder. Cognition, 113(1), 37-44.

HansImayr, S., Sauseng, P., Doppelmayr, M., Schabus, M., \& Klimesch, W. (2005). Increasing Individual Upper Alpha Power by Neurofeedback Improves Cognitive Performance in Human Subjects. Applied Psychophysiology and Biofeedback, 30(1), 1-10.

Happé, F. (2005). The weak central coherence account of autism. Handbook of Autism and Pervasive Developmental Disorders, Volume 1, Third Edition, 640-649.

Hari, R., Hämäläinen, M., \& Joutsiniemi, S.-L. (1989). Neuromagnetic steady-state responses to auditory stimuli. The Journal of the Acoustical Society of America, 86(3), 1033-1039.

Hartwright, C. E., Apperly, I. A., \& Hansen, P. C. (2012). Multiple roles for executive control in belief-desire reasoning: Distinct neural networks are recruited for self perspective inhibition and complexity of reasoning. Neurolmage, 61(4), 921-930.

Hartwright, C. E., Apperly, I. A., \& Hansen, P. C. (2015). The special case of self-perspective inhibition in mental, but not non-mental, representation. Neuropsychologia, 67, 183-192.

Hartwright, C. E., Hansen, P. C., \& Apperly, I. A. (2016). Current knowledge on the role of the Inferior Frontal Gyrus in Theory of Mind - A commentary on Schurz and Tholen (2016). Cortex, 85, 133136.

Hashemi, E., Ariza, J., Rogers, H., Noctor, S. C., \& Martínez-Cerdeño, V. (2016). The Number of Parvalbumin-Expressing Interneurons Is Decreased in the Medial Prefrontal Cortex in Autism. Cerebral Cortex, bhw021.

Hazen, E. P., Stornelli, J. L., O'Rourke, J. A., Koesterer, K., \& McDougle, C. J. (2014). Sensory symptoms in autism spectrum disorders. Harvard Review of Psychiatry, 22(2), 112-124.

Heilbron, M., \& Chait, M. (2017). Great expectations: is there evidence for predictive coding in auditory cortex? Neuroscience. 
Helfrich, R. F., Breska, A., \& Knight, R. T. (2019). Neural Entrainment and Network Resonance in Support of Top-down guided Attention. Current Opinion in Psychology.

Heusser, A. C., Poeppel, D., Ezzyat, Y., \& Davachi, L. (2016). Episodic sequence memory is supported by a theta-gamma phase code. Nature Neuroscience, 19(10), 1374-1380.

Hillebrand, A., \& Barnes, G. R. (2005). Beamformer analysis of MEG data. International Review of Neurobiology, 68, 149-171.

Hirsh, I. J., \& Watson, C. S. (1996). Auditory Psychophysics and Perception. Annual Review of Psychology, $47(1), 461-484$.

Hoogenboom, N., Schoffelen, J.-M., Oostenveld, R., Parkes, L. M., \& Fries, P. (2006a). Localizing human visual gamma-band activity in frequency, time and space. Neuroimage, 29(3), 764-773.

Hoogenboom, N., Schoffelen, J.-M., Oostenveld, R., Parkes, L. M., \& Fries, P. (2006b). Localizing human visual gamma-band activity in frequency, time and space. Neuroimage, 29(3), 764-773.

Hughes, H. C., Darcey, T. M., Barkan, H. I., Williamson, P. D., Roberts, D. W., \& Aslin, C. H. (2001). Responses of human auditory association cortex to the omission of an expected acoustic event. Neuroimage, 13(6), 1073-1089.

Hughes, J. R. (2007). Autism: the first firm finding= underconnectivity? Epilepsy \& Behavior, 11(1), 20-24.

Hyafil, A. (2015). Misidentifications of specific forms of cross-frequency coupling: three warnings. Frontiers in Neuroscience, 9.

Hyafil, A., Giraud, A.-L., Fontolan, L., \& Gutkin, B. (2015). Neural Cross-Frequency Coupling: Connecting Architectures, Mechanisms, and Functions. Trends in Neurosciences, 38(11), 725-740.

Igelström, K. M., \& Graziano, M. S. A. (n.d.). The inferior parietal lobule and temporoparietal junction: A network perspective. Neuropsychologia.

Javitt, D. C., Lee, M., Kantrowitz, J. T., \& Martinez, A. (2018). Mismatch negativity as a biomarker of theta band oscillatory dysfunction in schizophrenia. Schizophrenia Research, 191, 51-60.

Jenkinson, M., \& Smith, S. (2001). A global optimisation method for robust affine registration of brain images. Medical Image Analysis, 5(2), 143-156.

Jensen, O., \& Colgin, L. L. (2007). Cross-frequency coupling between neuronal oscillations. Trends in Cognitive Sciences, 11(7), 267-269.

Jensen, O., \& Mazaheri, A. (2010). Shaping functional architecture by oscillatory alpha activity: gating by inhibition. Frontiers in Human Neuroscience, 4, 186.

Jensen, O., Spaak, E., \& Park, H. (2016). Discriminating valid from spurious indices of phase-amplitude coupling. ENeuro, 3(6), ENEURO-0334.

Jiang, H., Bahramisharif, A., van Gerven, M. A., \& Jensen, O. (2015). Measuring directionality between neuronal oscillations of different frequencies. Neuroimage, 118, 359-367.

Jobs, E. N., Falck-Ytter, T., \& Bölte, S. (2018). Local and Global Visual Processing in 3-Year-Olds With and Without Autism. Journal of Autism and Developmental Disorders, 1-9.

Johnson, B. W., Crain, S., Thornton, R., Tesan, G., \& Reid, M. (2010). Measurement of brain function in pre-school children using a custom sized whole-head MEG sensor array. Clinical Neurophysiology, 121(3), 340-349.

Jones, R. S., Quigney, C., \& Huws, J. C. (2003). First-hand accounts of sensory perceptual experiences in autism: A qualitative analysis. Journal of Intellectual \& Developmental Disability, 28(2), 112-121.

Just, M. A., Cherkassky, V. L., Keller, T. A., Kana, R. K., \& Minshew, N. J. (2007). Functional and anatomical cortical underconnectivity in autism: evidence from an FMRI study of an executive function task and corpus callosum morphometry. Cerebral Cortex, 17(4), 951-961.

Kado, H., Higuchi, M., Shimogawara, M., Haruta, Y., Adachi, Y., Kawai, J., ... Uehara, G. (1999). Magnetoencephalogram systems developed at KIT. IEEE Transactions on Applied Superconductivity, 9(2), 4057-4062.

Kafaligonul, H., Breitmeyer, B. G., \& Öğmen, H. (2015). Feedforward and feedback processes in vision. Frontiers in Psychology, 6.

Kaldy, Z., Giserman, I., Carter, A. S., \& Blaser, E. (2016). The Mechanisms Underlying the ASD Advantage in Visual Search. Journal of Autism and Developmental Disorders, 46(5), 1513-1527.

Kana, R. K., Keller, T. A., Minshew, N. J., \& Just, M. A. (2007). Inhibitory control in high-functioning autism: decreased activation and underconnectivity in inhibition networks. Biological Psychiatry, 62(3), 198-206.

Kenny, L., Hattersley, C., Molins, B., Buckley, C., Povey, C., \& Pellicano, E. (2016). Which terms should be used to describe autism? Perspectives from the UK autism community. Autism, 20(4), 442-462. Keown, C. L., Shih, P., Nair, A., Peterson, N., Mulvey, M. E., \& Müller, R.-A. (2013). Local 
Functional Overconnectivity in Posterior Brain Regions Is Associated with Symptom Severity in Autism Spectrum Disorders. Cell Reports, 5(3), 567-572.

Kerkoerle, T. van, Self, M. W., Dagnino, B., Gariel-Mathis, M.-A., Poort, J., Togt, C. van der, \& Roelfsema, P. R. (2014). Alpha and gamma oscillations characterize feedback and feedforward processing in monkey visual cortex. Proceedings of the National Academy of Sciences, 111(40), 14332-14341.

Kessler, K., Seymour, R. A., \& Rippon, G. (2016). Brain oscillations and connectivity in autism spectrum disorders (ASD): new approaches to methodology, measurement and modelling. Neuroscience \& Biobehavioral Reviews, 71, 601-620.

Kessler, Klaus, Cao, L., O'Shea, K. J., \& Wang, H. (2014). A cross-culture, cross-gender comparison of perspective taking mechanisms. Proceedings of the Royal Society of London B: Biological Sciences, 281(1785), 20140388.

Kessler, Klaus, \& Rutherford, H. (2010). The Two Forms of Visuo-Spatial Perspective Taking are Differently Embodied and Subserve Different Spatial Prepositions. Frontiers in Psychology, 1, 213.

Kessler, Klaus, \& Thomson, L. A. (2010). The embodied nature of spatial perspective taking: embodied transformation versus sensorimotor interference. Cognition, 114(1), 72-88.

Khan, Michmizos, K., Tommerdahl, M., Ganesan, S., Kitzbichler, M. G., Zetino, M., ... Kenet, T. (2015). Somatosensory cortex functional connectivity abnormalities in autism show opposite trends, depending on direction and spatial scale. Brain, 138(5), 1394-1409.

Khan, S., Gramfort, A., Shetty, N. R., Kitzbichler, M. G., Ganesan, S., Moran, J. M., ... Kenet, T. (2013). Local and long-range functional connectivity is reduced in concert in autism spectrum disorders. Proceedings of the National Academy of Sciences, 110(8), 3107-3112.

Kirihara, K., Rissling, A. J., Swerdlow, N. R., Braff, D. L., \& Light, G. A. (2012). Hierarchical Organization of Gamma and Theta Oscillatory Dynamics in Schizophrenia. Biological Psychiatry, 71(10), 873-880.

Kitzbichler, M. G., Khan, S., Ganesan, S., Vangel, M. G., Herbert, M. R., Hämäläinen, M. S., \& Kenet, T. (2015). Altered Development and Multifaceted Band-Specific Abnormalities of Resting State Networks in Autism. Biological Psychiatry, 77(9), 794-804.

Klimesch, W. (2012a). Alpha-band oscillations, attention, and controlled access to stored information. Trends in Cognitive Sciences, 16(12), 606-617.

Klimesch, W. (2012b). Alpha-band oscillations, attention, and controlled access to stored information. Trends in Cognitive Sciences, 16(12), 606-617.

Klimesch, W., Sauseng, P., \& Gerloff, C. (2003). Enhancing cognitive performance with repetitive transcranial magnetic stimulation at human individual alpha frequency. European Journal of Neuroscience, 17(5), 1129-1133.

Kok, P., Bains, L. J., van Mourik, T., Norris, D. G., \& de Lange, F. P. (2016). Selective activation of the deep layers of the human primary visual cortex by top-down feedback. Current Biology, 26(3), 371-376.

Koshino, H., Kana, R. K., Keller, T. A., Cherkassky, V. L., Minshew, N. J., \& Just, M. A. (2008). fMRI investigation of working memory for faces in autism: visual coding and underconnectivity with frontal areas. Cerebral Cortex, 18(2), 289-300.

Kramer, M. A., Tort, A. B., \& Kopell, N. J. (2008). Sharp edge artifacts and spurious coupling in EEG frequency comodulation measures. Journal of Neuroscience Methods, 170(2), 352-357.

La Tour, T. D., Tallot, L., Grabot, L., Doyère, V., Van Wassenhove, V., Grenier, Y., \& Gramfort, A. (2017). Non-linear auto-regressive models for cross-frequency coupling in neural time series. PLoS Computational Biology, 13(12), e1005893.

Lawson, R. P., Aylward, J., Roiser, J. P., \& Rees, G. (2017). Adaptation of social and non-social cues to direction in adults with autism spectrum disorder and neurotypical adults with autistic traits. Developmental Cognitive Neuroscience.

Lawson, R. P., Mathys, C., \& Rees, G. (2017). Adults with autism overestimate the volatility of the sensory environment. Nature Neuroscience.

Lawson, R. P., Rees, G., \& Friston, K. J. (2014). An aberrant precision account of autism. Frontiers in Human Neuroscience, 8.

Le Van Quyen, M., Foucher, J., Lachaux, J.-P., Rodriguez, E., Lutz, A., Martinerie, J., \& Varela, F. J. (2001). Comparison of Hilbert transform and wavelet methods for the analysis of neuronal synchrony. Journal of Neuroscience Methods, 111(2), 83-98.

Leekam, S. R., Nieto, C., Libby, S. J., Wing, L., \& Gould, J. (2007). Describing the sensory abnormalities of children and adults with autism. Journal of Autism and Developmental Disorders, 37(5), 894-910.

Lega, B., Burke, J., Jacobs, J., \& Kahana, M. J. (2014). Slow-theta-to-gamma phase-amplitude coupling in human hippocampus supports the formation of new episodic memories. Cerebral Cortex, bhu232. 
Leung, R. C., Ye, A. X., Wong, S. M., Taylor, M. J., \& Doesburg, S. M. (2014). Reduced beta connectivity during emotional face processing in adolescents with autism. Molecular Autism, 5(1), 51.

Lieberman, M. D. (2007). Social cognitive neuroscience: a review of core processes. Annu. Rev. Psychol., 58, 259-289.

Lisman, J. E., \& Idiart, M. A. (1995). Storage of 7 plus/minus 2 short-term memories in oscillatory subcycles. Science, 267(5203), 1512.

Litvak, V., Mattout, J., Kiebel, S., Phillips, C., Henson, R., Kilner, J., ... others. (2011). EEG and MEG data analysis in SPM8. Computational Intelligence and Neuroscience, 2011.

Lopes da Silva, F. (2013). EEG and MEG: Relevance to Neuroscience. Neuron, 80(5), 1112-1128.

Loth, E., Murphy, D. G., \& Spooren, W. (2016). Defining precision medicine approaches to autism spectrum disorders: concepts and challenges. Frontiers in Psychiatry, 7, 188.

Lozano-Soldevilla, D., ter Huurne, N., \& Oostenveld, R. (2016). Neuronal oscillations with non-sinusoidal morphology produce spurious phase-to-amplitude coupling and directionality. Frontiers in Computational Neuroscience, 10.

Mamashli, F., Khan, S., Bharadwaj, H., Losh, A., Pawlyszyn, S. M., Hämäläinen, M. S., \& Kenet, T. (2018a). Maturational trajectories of local and long-range functional connectivity in autism during face processing. Human Brain Mapping, 39(10), 4094-4104.

Mamashli, F., Khan, S., Bharadwaj, H., Losh, A., Pawlyszyn, S. M., Hämäläinen, M. S., \& Kenet, T. (2018b). Maturational trajectories of local and long-range functional connectivity in autism during face processing. Human Brain Mapping, 39(10), 4094-4104.

Mamashli, F., Khan, S., Bharadwaj, H., Michmizos, K., Ganesan, S., Garel, K.-L. A., ... Kenet, T. (2017). Auditory processing in noise is associated with complex patterns of disrupted functional connectivity in autism spectrum disorder. Autism Research, 10(4), 631-647.

Manita, S., Suzuki, T., Homma, C., Matsumoto, T., Odagawa, M., Yamada, K., ... Sato, M. (2015). A topdown cortical circuit for accurate sensory perception. Neuron, 86(5), 1304-1316.

Maris, E., \& Oostenveld, R. (2007). Nonparametric statistical testing of EEG- and MEG-data. Journal of Neuroscience Methods, 164(1), 177-190.

Markov, N. T., Vezoli, J., Chameau, P., Falchier, A., Quilodran, R., Huissoud, C., ... Ullman, S. (2014). Anatomy of hierarchy: feedforward and feedback pathways in macaque visual cortex. Journal of Comparative Neurology, 522(1), 225-259.

Markram, K., \& Markram, H. (2010). The intense world theory-a unifying theory of the neurobiology of autism. Frontiers in Human Neuroscience, 4, 224.

Mars, R. B., Sallet, J., Schüffelgen, U., Jbabdi, S., Toni, I., \& Rushworth, M. F. (2012). Connectivity-based subdivisions of the human right "temporoparietal junction area": evidence for different areas participating in different cortical networks. Cerebral Cortex, 22(8), 1894-1903.

Martínez-Cancino, R., Heng, J., Delorme, A., Kreutz-Delgado, K., Sotero, R. C., \& Makeig, S. (2019). Measuring transient phase-amplitude coupling using local mutual information. Neurolmage, 185, 361-378.

Mathewson, K. E., Lleras, A., Beck, D. M., Fabiani, M., Ro, T., \& Gratton, G. (2011). Pulsed out of awareness: EEG alpha oscillations represent a pulsed-inhibition of ongoing cortical processing. Frontiers in Psychology, 2.

Maximo, J. O., Keown, C. L., Nair, A., \& Müller, R.-A. (2013). Approaches to local connectivity in autism using resting state functional connectivity MRI. Frontiers in Human Neuroscience, 7, 605.

May, M. (2004). Imaginal perspective switches in remembered environments: Transformation versus interference accounts. Cognitive Psychology, 48(2), 163-206.

May, P. J., \& Tiitinen, H. (2010). Mismatch negativity (MMN), the deviance-elicited auditory deflection, explained. Psychophysiology, 47(1), 66-122.

McCleery, J. P., Surtees, A. D. R., Graham, K. A., Richards, J. E., \& Apperly, I. A. (2011). The Neural and Cognitive Time Course of Theory of Mind. Journal of Neuroscience, 31(36), 12849-12854.

McFadden, K. L., Hepburn, S., Winterrowd, E., Schmidt, G. L., \& Rojas, D. C. (2012). Abnormalities in gamma-band responses to language stimuli in first-degree relatives of children with autism spectrum disorder: an MEG study. BMC Psychiatry, 12, 213.

McFadden, K. L., Steinmetz, S. E., Carroll, A. M., Simon, S. T., Wallace, A., \& Rojas, D. C. (2014). TestRetest Reliability of the $40 \mathrm{~Hz}$ EEG Auditory Steady-State Response. PLOS ONE, 9(1), e85748.

McPartland, J. C., \& Jeste, S. S. (2015). Connectivity in Context: Emphasizing Neurodevelopment in Autism Spectrum Disorder. Biological Psychiatry, 77(9), 772-774.

Medina, J., Jax, S. A., \& Coslett, H. B. (2009). Two-component models of reaching: Evidence from deafferentation in a Fitts' law task. Neuroscience Letters, 451(3), 222-226. 
Mejias, J. F., Murray, J. D., Kennedy, H., \& Wang, X.-J. (2016a). Feedforward and feedback frequencydependent interactions in a large-scale laminar network of the primate cortex. Science Advances, 2(11), e1601335.

Mejias, J. F., Murray, J. D., Kennedy, H., \& Wang, X.-J. (2016b). Feedforward and feedback frequencydependent interactions in a large-scale laminar network of the primate cortex. Science Advances, 2(11).

Michalareas, G., Vezoli, J., Van Pelt, S., Schoffelen, J.-M., Kennedy, H., \& Fries, P. (2016). Alpha-beta and gamma rhythms subserve feedback and feedforward influences among human visual cortical areas. Neuron, 89(2), 384-397.

Michalareas, G., Vezoli, J., van Pelt, S., Schoffelen, J.-M., Kennedy, H., \& Fries, P. (2016). Alpha-Beta and Gamma Rhythms Subserve Feedback and Feedforward Influences among Human Visual Cortical Areas. Neuron.

Michie, P. T. (2001). What has MMN revealed about the auditory system in schizophrenia? International Journal of Psychophysiology, 42(2), 177-194.

Milne, E., Scope, A., Pascalis, O., Buckley, D., \& Makeig, S. (2009). Independent Component Analysis Reveals Atypical Electroencephalographic Activity During Visual Perception in Individuals with Autism. Biological Psychiatry, 65(1), 22-30.

Moll, H., \& Tomasello, M. (2006). Level 1 perspective-taking at 24 months of age. British Journal of Developmental Psychology, 24(3), 603-613.

Moore, J. K., \& Linthicum Jr, F. H. (2007). The human auditory system: a timeline of development. International Journal of Audiology, 46(9), 460-478.

Moran, R. J., Stephan, K. E., Seidenbecher, T., Pape, H.-C., Dolan, R. J., \& Friston, K. J. (2009). Dynamic causal models of steady-state responses. Neurolmage, 44(3), 796-811.

Mottron, L., Dawson, M., Soulieres, I., Hubert, B., \& Burack, J. (2006). Enhanced perceptual functioning in autism: An update, and eight principles of autistic perception. Journal of Autism and Developmental Disorders, 36(1), 27-43.

Muckli, L., De Martino, F., Vizioli, L., Petro, L. S., Smith, F. W., Ugurbil, K., .. Yacoub, E. (2015). Contextual feedback to superficial layers of V1. Current Biology, 25(20), 2690-2695.

Müller, R.-A., Shih, P., Keehn, B., Deyoe, J. R., Leyden, K. M., \& Shukla, D. K. (2011). Underconnected, but how? A survey of functional connectivity MRI studies in autism spectrum disorders. Cerebral Cortex, 21(10), 2233-2243.

Murdaugh, D. L., Shinkareva, S. V., Deshpande, H. R., Wang, J., Pennick, M. R., \& Kana, R. K. (2012). Differential Deactivation during Mentalizing and Classification of Autism Based on Default Mode Network Connectivity. PLOS ONE, 7(11), e50064.

Muthukumaraswamy, S. D. (2013). High-frequency brain activity and muscle artifacts in MEG/EEG: a review and recommendations. Frontiers in Human Neuroscience, 7.

Muthukumaraswamy, S. D., Edden, R. A., Jones, D. K., Swettenham, J. B., \& Singh, K. D. (2009). Resting GABA concentration predicts peak gamma frequency and $\mathrm{fMRI}$ amplitude in response to visual stimulation in humans. Proceedings of the National Academy of Sciences, 106(20), 8356-8361.

Muthukumaraswamy, S. D., \& Singh, K. D. (2008). Spatiotemporal frequency tuning of BOLD and gamma band MEG responses compared in primary visual cortex. Neurolmage, 40(4), 1552-1560.

Muthukumaraswamy, S. D., Singh, K. D., Swettenham, J. B., \& Jones, D. K. (2010). Visual gamma oscillations and evoked responses: variability, repeatability and structural MRI correlates. Neuroimage, 49(4), 3349-3357.

Näätänen, R. (1995). The mismatch negativity: a powerful tool for cognitive neuroscience. Ear and Hearing, 16(1), 6-18.

Näätänen, R., Paavilainen, P., Rinne, T., \& Alho, K. (2007). The mismatch negativity (MMN) in basic research of central auditory processing: a review. Clinical Neurophysiology, 118(12), 2544-2590.

Nadalin, J. K., Martinet, L.-E., Widge, A. S., Cash, S. S., Eden, U., \& Kramer, M. A. (2019). A statistical modeling framework to assess cross-frequency coupling while accounting for confounding effects. BioRxiv, 519470.

Nolte, G. (2003). The magnetic lead field theorem in the quasi-static approximation and its use for magnetoencephalography forward calculation in realistic volume conductors. Physics in Medicine and Biology, 48(22), 3637-3652.

Nolte, G., Bai, O., Wheaton, L., Mari, Z., Vorbach, S., \& Hallett, M. (2004). Identifying true brain interaction from EEG data using the imaginary part of coherency. Clinical Neurophysiology: Official Journal of the International Federation of Clinical Neurophysiology, 115(10), 2292-2307. 
Nomi, J. S., \& Uddin, L. Q. (2015). Developmental changes in large-scale network connectivity in autism. Neurolmage: Clinical, 7, 732-741.

Oakley, B. F., Brewer, R., Bird, G., \& Catmur, C. (2016). Theory of mind is not theory of emotion: A cautionary note on the Reading the Mind in the Eyes Test. Journal of Abnormal Psychology, 125(6), 818.

Onslow, A. C. E., Jones, M. W., \& Bogacz, R. (2014). A canonical circuit for generating phase-amplitude coupling. PloS One, 9(8), e102591.

Oostenveld, R., Fries, P., Maris, E., \& Schoffelen, J.-M. (2010). FieldTrip: open source software for advanced analysis of MEG, EEG, and invasive electrophysiological data. Computational Intelligence and Neuroscience, 2011.

Oostenveld, R., Fries, P., Maris, E., \& Schoffelen, J.-M. (2011). FieldTrip: open source software for advanced analysis of MEG, EEG, and invasive electrophysiological data. Computational Intelligence and Neuroscience, 2011, 1.

Orekhova, E. V., Stroganova, T. A., Nygren, G., Tsetlin, M. M., Posikera, I. N., Gillberg, C., \& Elam, M. (2007). Excess of high frequency electroencephalogram oscillations in boys with autism. Biological Psychiatry, 62(9), 1022-1029.

O'riordan, M. A. (2004). Superior visual search in adults with autism. Autism, 8(3), 229-248.

O'Riordan, M. A., Plaisted, K. C., Driver, J., \& Baron-Cohen, S. (2001). Superior visual search in autism. Journal of Experimental Psychology: Human Perception and Performance, 27(3), 719.

Otten, M., Seth, A. K., \& Pinto, Y. (2017). A social Bayesian brain: How social knowledge can shape visual perception. Brain and Cognition, 112, 69-77.

Özkurt, T. E., \& Schnitzler, A. (2011). A critical note on the definition of phase-amplitude cross-frequency coupling. Journal of Neuroscience Methods, 201(2), 438-443.

Paakki, J.-J., Rahko, J., Long, X., Moilanen, I., Tervonen, O., Nikkinen, J., ... others. (2010). Alterations in regional homogeneity of resting-state brain activity in autism spectrum disorders. Brain Research, 1321, 169-179.

Padmanabhan, A., Lynch, C. J., Schaer, M., \& Menon, V. (2017). The Default Mode Network in Autism. Biological Psychiatry: Cognitive Neuroscience and Neuroimaging, 2(6), 476-486.

Palmer, C. J., Lawson, R. P., \& Hohwy, J. (2017). Bayesian approaches to autism: Towards volatility, action, and behavior. Psychological Bulletin, 143(5), 521.

Palmer, C. J., Seth, A. K., \& Hohwy, J. (2015). The felt presence of other minds: Predictive processing, counterfactual predictions, and mentalising in autism. Consciousness and Cognition, 36, 376-389.

Palva, J. M., Palva, S., \& Kaila, K. (2005). Phase synchrony among neuronal oscillations in the human cortex. Journal of Neuroscience, 25(15), 3962-3972.

Palva, S., \& Palva, J. M. (2011). Functional roles of alpha-band phase synchronization in local and largescale cortical networks. Front Psychol, 2, 204.

Pantev, C., Roberts, L. E., Elbert, T., Roß, B., \& Wienbruch, C. (1996). Tonotopic organization of the sources of human auditory steady-state responses. Hearing Research, 101(1), 62-74.

Pantev, Christo. (1995). Evoked and induced gamma-band activity of the human cortex. Brain Topography, 7(4), 321-330.

Park, H., Lee, D. S., Kang, E., Kang, H., Hahm, J., Kim, J. S., ... Jensen, O. (2016). Formation of visual memories controlled by gamma power phase-locked to alpha oscillations. Scientific Reports, 6 , 28092.

Pearson, A., Marsh, L., Ropar, D., \& Hamilton, A. (2016). Cognitive Mechanisms underlying visual perspective taking in typical and ASC children. Autism Research, 9(1), 121-130.

Pearson, A., Ropar, D., \& Hamilton, A. F. de C. (2013). A review of visual perspective taking in autism spectrum disorder.

Peiker, I., David, N., Schneider, T. R., Nolte, G., Schöttle, D., \& Engel, A. K. (2015). Perceptual Integration Deficits in Autism Spectrum Disorders Are Associated with Reduced Interhemispheric GammaBand Coherence. The Journal of Neuroscience, 35(50), 16352-16361.

Peiker, I., Schneider, T. R., Milne, E., Schöttle, D., Vogeley, K., Münchau, A., .. David, N. (2015). Stronger Neural Modulation by Visual Motion Intensity in Autism Spectrum Disorders. PLoS ONE, 10(7), e0132531.

Peirce, J. W. (2009). Generating stimuli for neuroscience using PsychoPy. Frontiers in Neuroinformatics, 2 , 10.

Pekkonen, E., Jousmäki, V., Reinikainen, K., \& Partanen, J. (1995). Automatic auditory discrimination is impaired in Parkinson's disease. Clinical Neurophysiology, 95(1), 47-52. 
Pellicano, E., \& Burr, D. (2012). When the world becomes 'too real': a Bayesian explanation of autistic perception. Trends in Cognitive Sciences, 16(10), 504-510.

Pellicano, E., Gibson, L., Maybery, M., Durkin, K., \& Badcock, D. R. (2005). Abnormal global processing along the dorsal visual pathway in autism: a possible mechanism for weak visuospatial coherence? Neuropsychologia, 43(7), 1044-1053.

Picci, G., Gotts, S. J., \& Scherf, K. S. (2016). A theoretical rut: revisiting and critically evaluating the generalized under/over-connectivity hypothesis of autism. Developmental Science, 19(4), 524-549.

Pollonini, L., Patidar, U., Situ, N., Rezaie, R., Papanicolaou, A. C., \& Zouridakis, G. (2010). Functional connectivity networks in the autistic and healthy brain assessed using Granger causality. In Engineering in Medicine and Biology Society (EMBC), 2010 Annual International Conference of the IEEE (pp. 1730-1733). IEEE.

Port, R. G., Edgar, J. C., Ku, M., Bloy, L., Murray, R., Blaskey, L., ... Roberts, T. P. (2016). Maturation of auditory neural processes in autism spectrum disorder-A longitudinal MEG study. Neurolmage: Clinical, 11, 566-577.

Raij, T., McEvoy, L., Mäkelä, J. P., \& Hari, R. (1997). Human auditory cortex is activated by omissions of auditory stimuli. Brain Research, 745(1-2), 134-143.

Ramsey, R., Hansen, P., Apperly, I., \& Samson, D. (2013). Seeing it my way or your way: frontoparietal brain areas sustain viewpoint-independent perspective selection processes. Journal of Cognitive Neuroscience, 25(5), 670-684.

Rao, R. P., \& Ballard, D. H. (1999). Predictive coding in the visual cortex: a functional interpretation of some extra-classical receptive-field effects. Nature Neuroscience, 2(1), 79.

Raven, J. C., \& Court, J. H. (1998). Raven's progressive matrices and vocabulary scales. Oxford Psychologists Press Oxford, UK.

Recasens, M., Gross, J., \& Uhlhaas, P. J. (2018). Low-Frequency Oscillatory Correlates of Auditory Predictive Processing in Cortical-Subcortical Networks: A MEG-Study. Scientific Reports, 8(1), 14007.

Recasens, M., \& Uhlhaas, P. J. (2017). Test-retest reliability of the magnetic mismatch negativity response to sound duration and omission deviants. Neurolmage.

Richter, C. G., Thompson, W. H., Bosman, C. A., \& Fries, P. (2017). Top-down beta enhances bottom-up gamma. Journal of Neuroscience, 3771-16.

Rippon, G., Brock, J., Brown, C., \& Boucher, J. (2007). Disordered connectivity in the autistic brain: challenges for the 'new psychophysiology'. International Journal of Psychophysiology, 63(2), 164172.

Roberts, T. P., Khan, S. Y., Rey, M., Monroe, J. F., Cannon, K., Blaskey, L., ... others. (2010). MEG detection of delayed auditory evoked responses in autism spectrum disorders: towards an imaging biomarker for autism. Autism Research, 3(1), 8-18.

Roberts, T. P. L., Cannon, K. M., Tavabi, K., Blaskey, L., Khan, S. Y., Monroe, J. F., ... Edgar, J. C. (2011). Auditory Magnetic Mismatch Field Latency: A Biomarker for Language Impairment in Autism. Biological Psychiatry, 70(3), 263-269.

Roberts, T. P., Paulson, D. N., Hirschkoff, G., Pratt, K., Mascarenas, A., Miller, P., ... Power, W. (2014). Artemis 123: development of a whole-head infant and young child MEG system. Frontiers in Human Neuroscience, 8, 99.

Robertson, A. E., \& Simmons, D. R. (2013). The relationship between sensory sensitivity and autistic traits in the general population. Journal of Autism and Developmental Disorders, 43(4), 775-784.

Robertson, A., \& Simmons, D. R. (2013). The Relationship between Sensory Sensitivity and Autistic Traits in the General Population. Journal of Autism and Developmental Disorders, 43(4), 775-784.

Robertson, C. E., \& Baron-Cohen, S. (2017). Sensory perception in autism. Nature Reviews Neuroscience, 18(11), 671.

Robertson, C., Kravitz, D. J., Freyberg, J., Baron-Cohen, S., \& Baker, C. I. (2013). Slower rate of binocular rivalry in autism. Journal of Neuroscience, 33(43), 16983-16991.

Robertson, C., Thomas, C., Kravitz, D. J., Wallace, G. L., Baron-Cohen, S., Martin, A., \& Baker, C. I. (2014). Global motion perception deficits in autism are reflected as early as primary visual cortex. Brain, 137(9), 2588-2599.

Rojas, D. C., Maharajh, K., Teale, P. D., Kleman, M. R., Benkers, T. L., Carlson, J. P., \& Reite, M. L. (2006). Development of the $40 \mathrm{~Hz}$ steady state auditory evoked magnetic field from ages 5 to 52 . Clinical Neurophysiology, 117(1), 110-117.

Rojas, D. C., Maharajh, K., Teale, P., \& Rogers, S. J. (2008). Reduced neural synchronization of gammaband MEG oscillations in first-degree relatives of children with autism. BMC Psychiatry, 8(1), 66. 
Rojas, D. C., Teale, P. D., Maharajh, K., Kronberg, E., Youngpeter, K., Wilson, L. B., ... Hepburn, S. (2011a). Transient and steady-state auditory gamma-band responses in first-degree relatives of people with autism spectrum disorder. Molecular Autism, 2(1), 1.

Rojas, D. C., Teale, P. D., Maharajh, K., Kronberg, E., Youngpeter, K., Wilson, L. B., ... Hepburn, S. (2011b). Transient and steady-state auditory gamma-band responses in first-degree relatives of people with autism spectrum disorder. Molecular Autism, 2(1), 11.

Rojas, D. C., \& Wilson, L. B. (2014). Y-band abnormalities as markers of autism spectrum disorders. Biomarkers in Medicine, 8(3), 353-368.

Ross, B., Herdman, A. T., \& Pantev, C. (2005). Right hemispheric laterality of human $40 \mathrm{~Hz}$ auditory steady-state responses. Cerebral Cortex, 15(12), 2029-2039.

Ross, B., Picton, T. W., Herdman, A. T., \& Pantev, C. (2004). The effect of attention on the auditory steadystate response. Neurology \& Clinical Neurophysiology: NCN, 2004, 22-22.

Rousselet, G. A., \& Pernet, C. R. (2012). Improving standards in brain-behavior correlation analyses. Frontiers in Human Neuroscience, 6.

Rubenstein, J. L. R., \& Merzenich, M. M. (2003). Model of autism: increased ratio of excitation/inhibition in key neural systems. Genes, Brain and Behavior, 2(5), 255-267.

Samson, D., Apperly, I. A., Kathirgamanathan, U., \& Humphreys, G. W. (2005). Seeing it my way: a case of a selective deficit in inhibiting self-perspective. Brain, 128(5), 1102-1111.

Samson, D., Houthuys, S., \& Humphreys, G. W. (2015). Self-perspective inhibition deficits cannot be explained by general executive control difficulties. Cortex, 70, 189-201.

SanMiguel, I., Widmann, A., Bendixen, A., Trujillo-Barreto, N., \& Schröger, E. (2013). Hearing silences: human auditory processing relies on preactivation of sound-specific brain activity patterns. Journal of Neuroscience, 33(20), 8633-8639.

Santarelli, R., Maurizi, M., Conti, G., Ottaviani, F., Paludetti, G., \& Pettorossi, V. E. (1995). Generation of human auditory steady-state responses (SSRs). II: Addition of responses to individual stimuli. Hearing Research, 83(1-2), 9-18.

Santiesteban, I., Banissy, M. J., Catmur, C., \& Bird, G. (2012). Enhancing social ability by stimulating right temporoparietal junction. Current Biology, 22(23), 2274-2277.

Sauer, A., Zeev-Wolf, M., Recasens, M., Wacongne, C., Wibral, M., Helbling, S., ... others. (2017). Impairment in predictive processes during auditory mismatch negativity in ScZ: Evidence from event-related fields. Human Brain Mapping.

Sauseng, P., Klimesch, W., Gruber, W. R., Hanslmayr, S., Freunberger, R., \& Doppelmayr, M. (2007). Are event-related potential components generated by phase resetting of brain oscillations? A critical discussion. Neuroscience, 146(4), 1435-1444.

Scheffer-Teixeira, R., \& Tort, A. B. (2016). On cross-frequency phase-phase coupling between theta and gamma oscillations in the hippocampus. ELife, 5, e20515.

Schoffelen, J.-M., Hultén, A., Lam, N., Marquand, A. F., Uddén, J., \& Hagoort, P. (2017). Frequencyspecific directed interactions in the human brain network for language. Proceedings of the National Academy of Sciences, 114(30), 8083-8088.

Schurz, M., Aichhorn, M., Martin, A., \& Perner, J. (2013). Common brain areas engaged in false belief reasoning and visual perspective taking: a meta-analysis of functional brain imaging studies. Frontiers in Human Neuroscience, 7.

Sevgi, M., Diaconescu, A. O., Tittgemeyer, M., \& Schilbach, L. (2016). Social Bayes: Using Bayesian Modeling to Study Autistic Trait-Related Differences in Social Cognition. Biological Psychiatry, 80(2), 112-119.

Seymour, R. A., Rippon, G., \& Kessler, K. (2017). The Detection of Phase Amplitude Coupling during Sensory Processing. Frontiers in Neuroscience, 11, 487.

Seymour, R. A., Wang, H., Rippon, G., \& Kessler, K. (2018). Oscillatory networks of high-level mental alignment: A perspective-taking MEG study. Neurolmage, 177, 98-107.

Siegel, M., Donner, T. H., Oostenveld, R., Fries, P., \& Engel, A. K. (2007). High-Frequency Activity in Human Visual Cortex Is Modulated by Visual Motion Strength. Cerebral Cortex, 17(3), 732-741.

Simmons, D. R., Robertson, A. E., McKay, L. S., Toal, E., McAleer, P., \& Pollick, F. E. (2009). Vision in autism spectrum disorders. Vision Research, 49(22), 2705-2739.

Simon, D. M., \& Wallace, M. T. (2016). Dysfunction of sensory oscillations in Autism Spectrum Disorder. Neuroscience \& Biobehavioral Reviews, 68, 848-861.

Singer, W., \& Gray, C. M. (1995). Visual feature integration and the temporal correlation hypothesis. Annual Review of Neuroscience, 18(1), 555-586. 
Snijders, T. M., Milivojevic, B., \& Kemner, C. (2013). Atypical excitation-inhibition balance in autism captured by the gamma response to contextual modulation. Neuroimage: Clinical, 3, 65-72.

Sowden, S., \& Catmur, C. (2015). The role of the right temporoparietal junction in the control of imitation. Cerebral Cortex, 25(4), 1107-1113.

Spaak, E., Bonnefond, M., Maier, A., Leopold, D. A., \& Jensen, O. (2012). Layer-specific entrainment of gamma-band neural activity by the alpha rhythm in monkey visual cortex. Current Biology, 22(24), 2313-2318.

Spence, S. J., \& Schneider, M. T. (2009). The role of epilepsy and epileptiform EEGs in autism spectrum disorders. Pediatric Research, 65(6), 599-606.

Spyropoulos, G., Bosman, C. A., \& Fries, P. (2018). A theta rhythm in macaque visual cortex and its attentional modulation. Proceedings of the National Academy of Sciences, 201719433.

Stenner, M.-P., Bauer, M., Haggard, P., Heinze, H.-J., \& Dolan, R. (2014). Enhanced alpha-oscillations in visual cortex during anticipation of self-generated visual stimulation. Journal of Cognitive Neuroscience. Retrieved from

Stokes, M. G., Wolff, M. J., \& Spaak, E. (2015). Decoding rich spatial information with high temporal resolution. Trends in Cognitive Sciences, 19(11), 636-638.

Stothart, G., \& Kazanina, N. (2013). Oscillatory characteristics of the visual mismatch negativity: what evoked potentials aren't telling us. Frontiers in Human Neuroscience, 7, 426.

Stroganova, T. A., Orekhova, E. V., Prokofyev, A. O., Tsetlin, M. M., Gratchev, V. V., Morozov, A. A., \& Obukhov, Y. V. (2012). High-frequency oscillatory response to illusory contour in typically developing boys and boys with autism spectrum disorders. Cortex, 48(6), 701-717.

Sun, L., Grützner, C., Bölte, S., Wibral, M., Tozman, T., Schlitt, S., ... Uhlhaas, P. J. (2012). Impaired gamma-band activity during perceptual organization in adults with autism spectrum disorders: evidence for dysfunctional network activity in frontal-posterior cortices. The Journal of Neuroscience, 32(28), 9563-9573.

Surtees, A., Apperly, I., \& Samson, D. (2013). The use of embodied self-rotation for visual and spatial perspective-taking. Frontiers in Human Neuroscience, 7.

Surtees, A. D. R., Butterfill, S. A., \& Apperly, I. A. (2012). Direct and indirect measures of Level-2 perspective-taking in children and adults. British Journal of Developmental Psychology, 30(1), 7586.

Sysoeva, O. V., Galuta, I. A., Davletshina, M. S., Orekhova, E. V., \& Stroganova, T. A. (2017). Abnormal Size-Dependent Modulation of Motion Perception in Children with Autism Spectrum Disorder (ASD). Frontiers in Neuroscience, 11.

Tadel, F., Baillet, S., Mosher, J. C., Pantazis, D., \& Leahy, R. M. (2011). Brainstorm: a user-friendly application for MEG/EEG analysis. Computational Intelligence and Neuroscience, 2011, 8.

Takesaki, N., Kikuchi, M., Yoshimura, Y., Hiraishi, H., Hasegawa, C., Kaneda, R., ... Minabe, Y. (2016). The Contribution of Increased Gamma Band Connectivity to Visual Non-Verbal Reasoning in Autistic Children: A MEG Study. PLOS ONE, 11(9), e0163133.

Tallon-Baudry, C., \& Bertrand, O. (1999). Oscillatory gamma activity in humans and its role in object representation. Trends in Cognitive Sciences, 3(4), 151-162.

Tan, H.-R., Gross, J., \& Uhlhaas, P. J. (2015). MEG—measured auditory steady-state oscillations show high test-retest reliability: A sensor and source-space analysis. Neuroimage, 122, 417-426.

Tan, J., \& Harris, P. L. (1991). Autistic children understand seeing and wanting. Development and Psychopathology, 3(2), 163-174.

Taulu, S., \& Simola, J. (2006). Spatiotemporal signal space separation method for rejecting nearby interference in MEG measurements. Physics in Medicine and Biology, 51(7), 1759.

Tecchio, F., Benassi, F., Zappasodi, F., Gialloreti, L. E., Palermo, M., Seri, S., \& Rossini, P. M. (2003). Auditory sensory processing in autism: a magnetoencephalographic study. Biological Psychiatry, 54(6), 647-654.

Tomasello, M., Carpenter, M., Call, J., Behne, T., \& Moll, H. (2005). Understanding and sharing intentions: the origins of cultural cognition. The Behavioral and Brain Sciences, 28(5), 675-691;

Tomchek, S. D., \& Dunn, W. (2007). Sensory processing in children with and without autism: a comparative study using the short sensory profile. American Journal of Occupational Therapy, 61(2), 190-200.

Tort, A. B., Fontanini, A., Kramer, M. A., Jones-Lush, L. M., Kopell, N. J., \& Katz, D. B. (2010). Cortical networks produce three distinct $7-12 \mathrm{~Hz}$ rhythms during single sensory responses in the awake rat. Journal of Neuroscience, 30(12), 4315-4324. 
Tort, A. B. L., Komorowski, R., Eichenbaum, H., \& Kopell, N. (2010). Measuring Phase-Amplitude Coupling Between Neuronal Oscillations of Different Frequencies. Journal of Neurophysiology, 104(2), 1195-1210.

Van Boxtel, J. J. A., \& Lu, H. (2013). A predictive coding perspective on autism spectrum disorders. Frontiers in Psychology, 4.

Van de Cruys, S., Evers, K., Van der Hallen, R., Van Eylen, L., Boets, B., de-Wit, L., \& Wagemans, J. (2014). Precise minds in uncertain worlds: predictive coding in autism. Psychological Review, 121(4), 649.

Van de Cruys, S., Van der Hallen, R., \& Wagemans, J. (2017). Disentangling signal and noise in autism spectrum disorder. Brain and Cognition, 112, 78-83.

Van der Meer, L., Groenewold, N. A., Nolen, W. A., Pijnenborg, M., \& Aleman, A. (2011). Inhibit yourself and understand the other: neural basis of distinct processes underlying Theory of Mind. Neuroimage, 56(4), 2364-2374.

van Driel, J., Cox, R., \& Cohen, M. X. (2015). Phase-clustering bias in phase-amplitude cross-frequency coupling and its removal. Journal of Neuroscience Methods, 254, 60-72.

Van Essen, D. C. (2012). Cortical cartography and Caret software. Neurolmage, 62(2), 757-764.

Van Essen, D. C., Ugurbil, K., Auerbach, E., Barch, D., Behrens, T. E. J., Bucholz, R., ... others. (2012). The Human Connectome Project: a data acquisition perspective. Neuroimage, 62(4), 2222-2231.

Van Overwalle, F. (2009). Social cognition and the brain: A meta-analysis. Human Brain Mapping, 30(3), 829-858.

Van Overwalle, F. (2011). A dissociation between social mentalizing and general reasoning. Neuroimage, 54(2), 1589-1599.

Van Veen, B. D., van Drongelen, W., Yuchtman, M., \& Suzuki, A. (1997). Localization of brain electrical activity via linearly constrained minimum variance spatial filtering. IEEE Transactions on Biomedical Engineering, 44(9), 867-880.

VanRullen, R., \& Koch, C. (2003). Visual Selective Behavior Can Be Triggered by a Feed-Forward Process. Journal of Cognitive Neuroscience, 15(2), 209-217.

Varela, F., Lachaux, J.-P., Rodriguez, E., \& Martinerie, J. (2001). The brainweb: phase synchronization and large-scale integration. Nature Reviews Neuroscience, 2(4), 229-239.

Vaz, A. P., Yaffe, R. B., Wittig Jr, J. H., Inati, S. K., \& Zaghloul, K. A. (2017). Dual origins of measured phase-amplitude coupling reveal distinct neural mechanisms underlying episodic memory in the human cortex. Neurolmage, 148, 148-159.

Vilidaite, G., Yu, M., \& Baker, D. H. (2017). Internal noise estimates correlate with autistic traits. Autism Research, 10(8), 1384-1391.

Vincent, J. L., Kahn, I., Snyder, A. Z., Raichle, M. E., \& Buckner, R. L. (2008). Evidence for a frontoparietal control system revealed by intrinsic functional connectivity. Journal of Neurophysiology, 100(6), 3328-3342.

Vissers, M. E., Cohen, M. X., \& Geurts, H. M. (2012). Brain connectivity and high functioning autism: a promising path of research that needs refined models, methodological convergence, and stronger behavioral links. Neuroscience \& Biobehavioral Reviews, 36(1), 604-625.

Vogeley, K., Bussfeld, P., Newen, A., Herrmann, S., Happé, F., Falkai, P., ... Zilles, K. (2001). Mind reading: neural mechanisms of theory of mind and self-perspective. Neuroimage, 14(1), 170-181.

Voloh, B., Valiante, T. A., Everling, S., \& Womelsdorf, T. (2015). Theta-gamma coordination between anterior cingulate and prefrontal cortex indexes correct attention shifts. Proceedings of the National Academy of Sciences, 112(27), 8457-8462.

von dem Hagen, E. A., Stoyanova, R. S., Baron-Cohen, S., \& Calder, A. J. (2012). Reduced functional connectivity within and between 'social'resting state networks in autism spectrum conditions. Social Cognitive and Affective Neuroscience, 8(6), 694-701.

Von Stein, A., Chiang, C., \& König, P. (2000). Top-down processing mediated by interareal synchronization. Proceedings of the National Academy of Sciences, 97(26), 14748-14753.

Voytek, B., Canolty, R. T., Shestyuk, A., Crone, N., Parvizi, J., \& Knight, R. T. (2010). Shifts in Gamma Phase-Amplitude Coupling Frequency from Theta to Alpha Over Posterior Cortex During Visual Tasks. Frontiers in Human Neuroscience, 4.

Voytek, B., Kayser, A. S., Badre, D., Fegen, D., Chang, E. F., Crone, N. E., ... D'esposito, M. (2015). Oscillatory dynamics coordinating human frontal networks in support of goal maintenance. Nature Neuroscience, 18(9), 1318-1324.

Voytek, B., \& Knight, R. T. (2015). Dynamic network communication as a unifying neural basis for cognition, development, aging, and disease. Biological Psychiatry, 77(12), 1089-1097. 
Wacongne, C., Changeux, J.-P., \& Dehaene, S. (2012). A neuronal model of predictive coding accounting for the mismatch negativity. Journal of Neuroscience, 32(11), 3665-3678.

Wacongne, C., Labyt, E., van Wassenhove, V., Bekinschtein, T., Naccache, L., \& Dehaene, S. (2011). Evidence for a hierarchy of predictions and prediction errors in human cortex. Proceedings of the National Academy of Sciences, 108(51), 20754-20759.

Wang, H., Callaghan, E., Gooding-Williams, G., McAllister, C., \& Kessler, K. (2016). Rhythm makes the world go round: An MEG-TMS study on the role of right TPJ theta oscillations in embodied perspective taking. Cortex, 75, 68-81.

Wang, L., Saalmann, Y. B., Pinsk, M. A., Arcaro, M. J., \& Kastner, S. (2012). Electrophysiological LowFrequency Coherence and Cross-Frequency Coupling Contribute to BOLD Connectivity. Neuron, 76(5), 1010-1020.

Wang, X., Chen, Y., \& Ding, M. (2008). Estimating Granger causality after stimulus onset: a cautionary note. Neuroimage, 41(3), 767-776.

Wass, S. (2011). Distortions and disconnections: disrupted brain connectivity in autism. Brain and Cognition, 75(1), 18-28.

Weaver, K. E., Wander, J. D., Ko, A. L., Casimo, K., Grabowski, T. J., Ojemann, J. G., \& Darvas, F. (2016). Directional patterns of cross frequency phase and amplitude coupling within the resting state mimic patterns of fMRI functional connectivity. Neurolmage, 128, 238-251.

Wilson, T. W., Rojas, D. C., Reite, M. L., Teale, P. D., \& Rogers, S. J. (2007). Children and adolescents with autism exhibit reduced MEG steady-state gamma responses. Biological Psychiatry, 62(3), 192-197.

Witkowski, M., Garcia-Cossio, E., Chander, B. S., Braun, C., Birbaumer, N., Robinson, S. E., \& Soekadar, S. R. (2016). Mapping entrained brain oscillations during transcranial alternating current stimulation (tACS). Neuroimage, 140, 89-98.

Womelsdorf, T., Schoffelen, J.-M., Oostenveld, R., Singer, W., Desimone, R., Engel, A. K., \& Fries, P. (2007). Modulation of neuronal interactions through neuronal synchronization. Science, 316(5831), $1609-1612$.

Wright, B., Alderson-Day, B., Prendergast, G., Bennett, S., Jordan, J., Whitton, C., ... others. (2012). Gamma activation in young people with autism spectrum disorders and typically-developing controls when viewing emotions on faces. PLoS One, 7(7), e41326.

Wu, Q., Chang, C.-F., Xi, S., Huang, I.-W., Liu, Z., Juan, C.-H., ... Fan, J. (2015). A critical role of temporoparietal junction in the integration of top-down and bottom-up attentional control. Human Brain Mapping, 36(11), 4317-4333.

Wyatte, D., Curran, T., \& O'Reilly, R. (2012). The limits of feedforward vision: recurrent processing promotes robust object recognition when objects are degraded. Journal of Cognitive Neuroscience, 24(11), 2248-2261.

Yabe, H., Tervaniemi, M., Reinikainen, K., \& Näätänen, R. (1997). Temporal window of integration revealed by MMN to sound omission. Neuroreport, 8(8), 1971-1974.

Zhang, S., Xu, M., Kamigaki, T., Do, J. P. H., Chang, W.-C., Jenvay, S., ... Dan, Y. (2014). Long-range and local circuits for top-down modulation of visual cortex processing. Science, 345(6197), 660-665.

Zikopoulos, B., \& Barbas, H. (2010). Changes in prefrontal axons may disrupt the network in autism. The Journal of Neuroscience, 30(44), 14595-14609.

Zikopoulos, B., \& Barbas, H. (2013). Altered neural connectivity in excitatory and inhibitory cortical circuits in autism. Frontiers in Human Neuroscience, 7, 609. 


\section{Appendix 1}

\section{Appendices}

Whilst many researchers choose 18 phase bins to compute PAC using the KL-MI-Tort approach, this number is somewhat arbitrary. We therefore altered the numbers of phase bins $(9,18$ and 36 phase bins) used in KL-MI-Tort computation, and investigated the effect on the ability to detect simulated PAC between $10-11 \mathrm{~Hz}$ phase and $50-70 \mathrm{~Hz}$ amplitude. All three phase bins were able to detect the alpha-gamma PAC, with no clear differences in the estimates of modulating phase or amplitude.
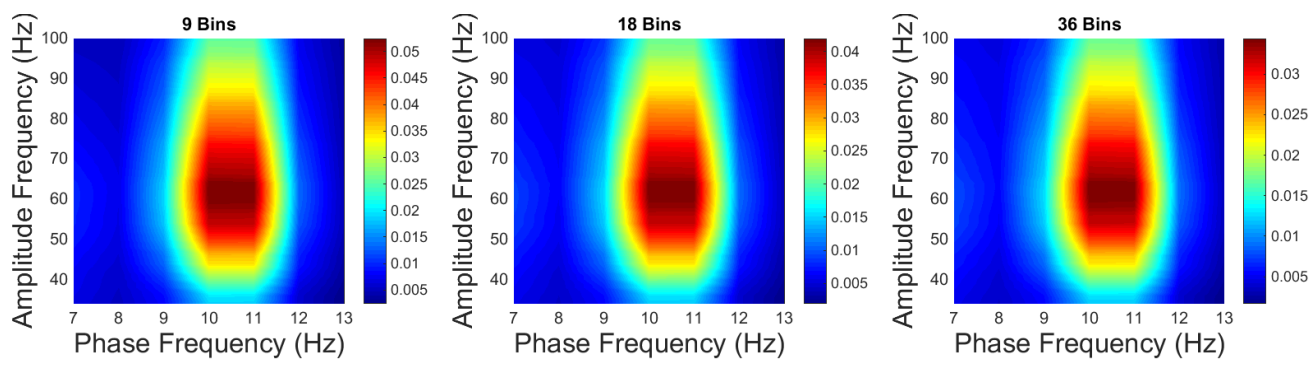

\section{Appendix 2}

Whole-brain statistical maps uncorrected for multiple comparisons, clicktrain $>$ baseline (0.3-1.5s time-period post-clicktrain onset compared with a $1.2 \mathrm{~s}$ baseline period). Only positive t-values are shown. Maximum t-values for each group and hemisphere are displayed.

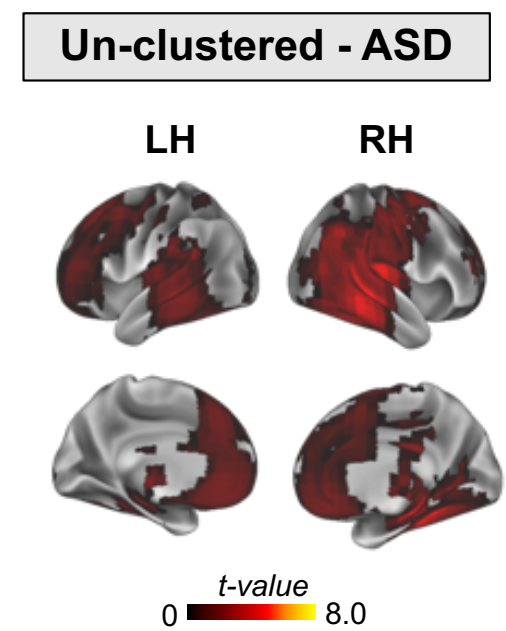

Max t-value: $\quad t=2.10$

$$
t=4.96
$$

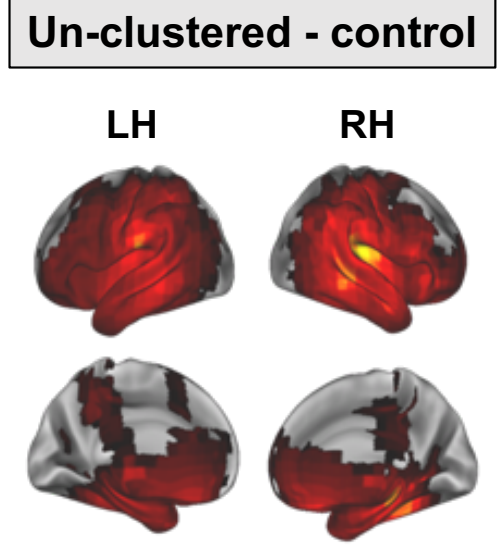

0 t-value 8.0

$t=6.24 \quad t=7.95$

LH = Left Hemisphere; RH = Right Hemisphere; ASD = Autism Spectrum Disorder

\section{Appendix 3}

Individual ERF plots (omiMMF and durMMF) are shown below for ASD and control groups. The red dot on each plot indicates the peak response per participant. 


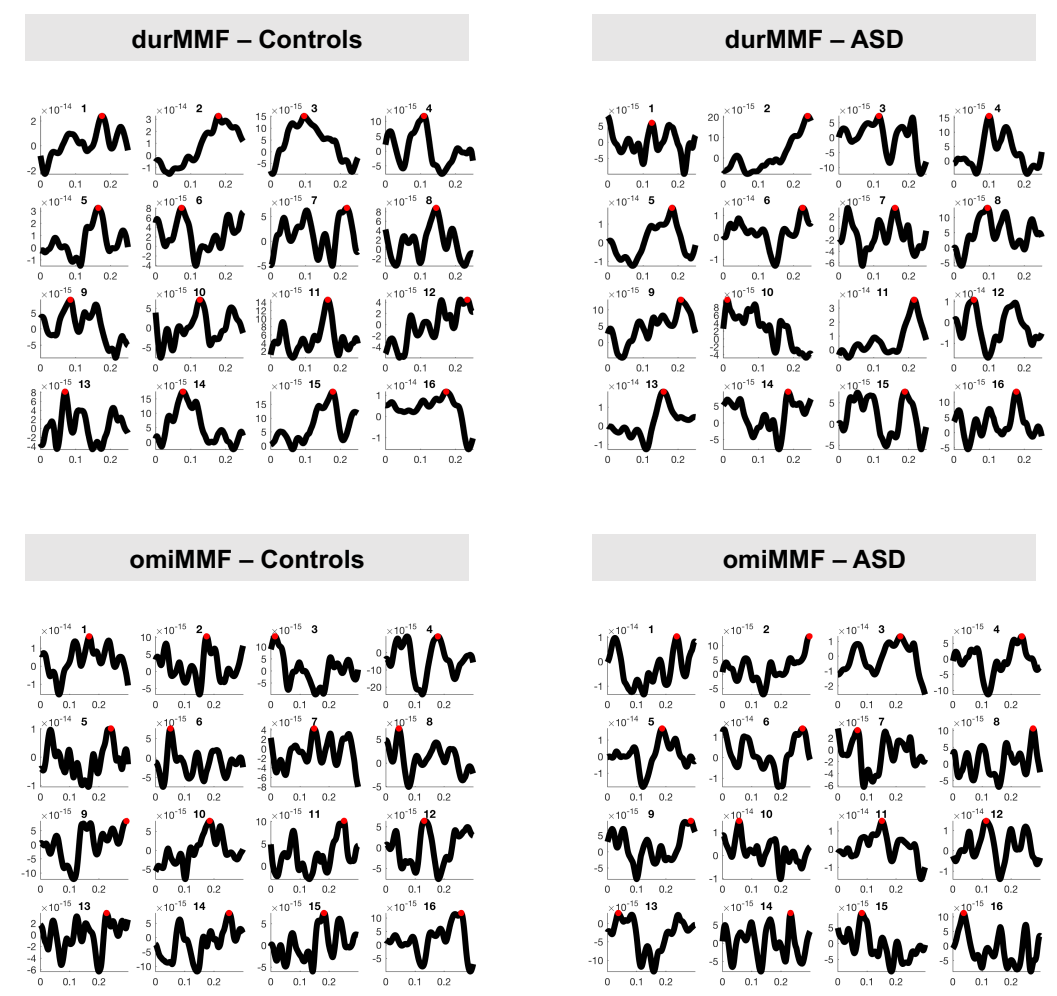

\section{Appendix 4}

Procedure for source localisation in Chapter 6. Sensor-level data from LR-160, LR-60 and their corresponding baseline periods were pooled to create an average covariance matrix. Using this matrix, source analysis was performed using a Dynamic Imaging of Coherent Sources (DICS) approach, resulting in a common spatial filter across all conditions.

This common filter was used to localise LR-160, LR-60 and baseline conditions separately using DICS (with the Fieldtrip ft_sourceanalysis option: $\mathrm{cfg} \cdot \mathrm{grid}$.filter = source_all.avg.filter). The resulting whole-brain power maps were baseline corrected and statistically compared (i.e. LR-160>LR-60) using cluster-based permutation testing.

For LR-160 and LR-60 trials, 'virtual-electrodes' were defined from regions of interest (ROIs). Sensor-level data were multiplied by the common spatial filter, using the specific grid-point corresponding to the MNI coordinates identified from the whole-brain analysis.

Sensor-Level Data:
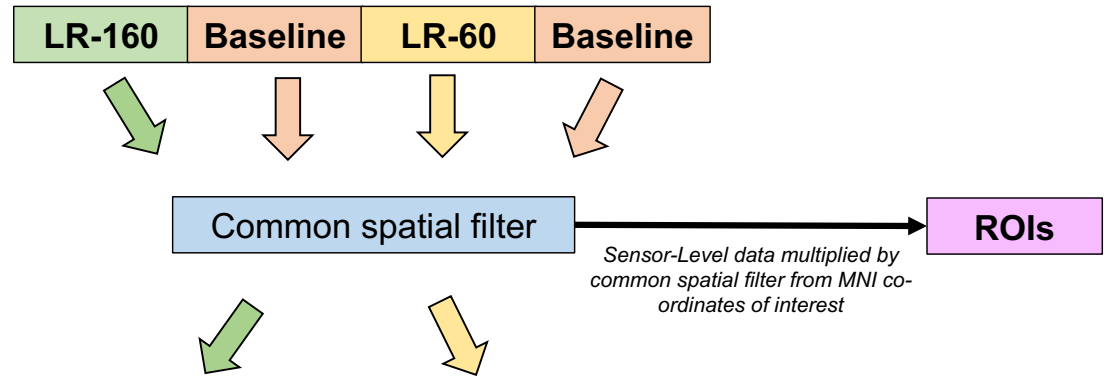

\begin{tabular}{l|l|l|l|l|}
\hline Source-Level Data: & LR-160 - Baseline & vs & LR-60 & Baseline \\
\hline
\end{tabular}

\section{Appendix 5}

Chapter 6 tables specifying co-ordinates of significant clusters. Table shows all local maxima separated by more than $0.2 \mathrm{~cm}$. Regions were automatically labelled using the 
AAL2 atlas. Montreal Neurological Institute (MNI) coordinates correspond to the left-right anterior-posterior and inferior-superior dimensions respectively.

\begin{tabular}{cccccc}
\hline Sign & $\begin{array}{c}\text { Source } \\
\text { Label in } \\
\text { Fig.5.5 }\end{array}$ & Brain Region & $\begin{array}{c}\text { MNI Co- } \\
\text { ordinates }\end{array}$ & $\begin{array}{c}\text { Maximum } \\
\text { t-value }\end{array}$ & $\begin{array}{c}\text { p- } \\
\text { value }\end{array}$ \\
\hline Positive & $\begin{array}{c}\text { Right } \\
\text { TPJ }\end{array}$ & Angular_R & $40-5836$ & 6.274 & .0025 \\
Positive & $\begin{array}{c}\text { Right } \\
\text { ACC }\end{array}$ & Cingulate_Ant_R & 123628 & 6.115 & .0093 \\
Positive & $\begin{array}{c}\text { Right } \\
\text { Lateral } \\
\text { PFC }\end{array}$ & Frontal_Inf_Tri_R & 523216 & 5.894 & .0341 \\
Negative & - & OFCpost_L & $-2824-20$ & -5.915 & .0428 \\
\hline
\end{tabular}

List of cortical sources for the LR-160>LR-60 theta-band power contrast shown in Fig. 6.5.

\begin{tabular}{ccccc}
\hline Sign & Brain Region & $\begin{array}{c}\text { MNI Co- } \\
\text { ordinates }\end{array}$ & $\begin{array}{c}\text { Maximum } \\
\text { t-value }\end{array}$ & $\begin{array}{c}\text { p- } \\
\text { value }\end{array}$ \\
\hline Positive & Frontal_Sup_Medial_R & 10480 & 3.169 & .0218 \\
Positive & SupraMarginal_R & $52-2424$ & 3.0948 & .0384 \\
Positive & Paracentral_Lobule_L & $-12-2076$ & 2.9418 & .0471 \\
Negative & Fusiform_R & $28-40-16$ & -4.7413 & .0154 \\
Negative & Temporal_Mid_L & $-600-26$ & -3.7938 & .0369 \\
\hline
\end{tabular}

List of cortical sources for the LR-160>LR-60 theta-band imaginary coherence contrast shown in Fig. 6.6.

\begin{tabular}{|c|c|c|c|c|c|}
\hline \multirow{2}{*}{\multicolumn{2}{|c|}{ Region Label }} & \multirow{2}{*}{ t-value } & \multicolumn{2}{|c|}{ MNI Coordinates } & \multirow[b]{2}{*}{$\mathbf{z}$} \\
\hline & & & $\mathbf{x}$ & $\mathbf{y}$ & \\
\hline \multirow[t]{6}{*}{ Positive } & R Middle Occipital Gyrus & 4.9953 & 46 & -80 & 8 \\
\hline & R Inferior Temporal Gyrus & 3.5761 & 52 & -56 & 0 \\
\hline & L Inferior Temporal Gyrus & 4.6888 & -58 & -16 & -24 \\
\hline & R Middle Frontal Gyrus & 3.6621 & 40 & 18 & 40 \\
\hline & R IFG (p. Opercularis) & 3.6522 & 42 & 12 & 16 \\
\hline & $\mathrm{R}$ Thalamus & 3.6391 & 14 & -22 & 10 \\
\hline
\end{tabular}

List of cortical sources for the LR-160>LR-60 theta-band power contrast (control group) shown in Fig. 6.9 left panel.

\begin{tabular}{llllcl}
\hline & & \multicolumn{3}{c}{ MNI Coordinates } \\
& Region Label & t-value & $\mathbf{x}$ & $\mathbf{y}$ & $\mathbf{z}$ \\
& & & & \\
\hline Positive & Temporal_Inf_R & 4.261 & 46 & -62 & -8
\end{tabular}




$\begin{array}{lllll}\text { Location not in atlas } & 4.0879 & 60 & -70 & 6 \\ \text { Frontal_Mid_2_R } & 3.5137 & 44 & 24 & 40 \\ \text { Location not in atlas } & 3.3639 & 36 & -30 & 0\end{array}$

List of cortical sources for the control > ASD theta-band power contrast shown in Fig. 6.9 right panel

\begin{tabular}{llllll}
\hline & & & \multicolumn{2}{c}{ MNI Coordinates } & \multicolumn{2}{c}{} \\
& Region Label & t-value & $\mathbf{x}$ & $\mathbf{y}$ & $\mathbf{z}$ \\
\hline Negative & L Cuneus & -4.069 & -4 & -94 & 34 \\
& L Middle Occipital Gyrus & -3.750 & -20 & -96 & 16 \\
& R Middle Temporal Gyrus & -3.740 & 44 & -70 & 8 \\
\hline
\end{tabular}

List of cortical sources for the LR-160>LR-60 alpha-band power contrast (ASD group) shown in Fig. 6.10 middle panel.

\begin{tabular}{llllll}
\hline & & \multicolumn{5}{l}{ MNI Coordinates } \\
& Region Label & t-value & $\mathbf{x}$ & $\mathbf{y}$ & $\mathbf{z}$ \\
\hline \multirow{2}{*}{ Negative } & Calcarine_L & -3.4805 & 4 & -96 & 2 \\
& Cuneus_L & -2.8423 & 2 & -88 & 34 \\
\hline
\end{tabular}

List of cortical sources for the control > ASD alpha-band power contrast shown in Fig. 6.10 right panel.

\section{Appendix 6}

In Chapter 6, Experiment 2, data from 4 participants (2 ASD, 2 control) were discarded due to a median RT greater than 2 standard deviations from the group median. Individual ASD participant RT data are plotted below for each experimental condition, with the individuals removed highlighted with a yellow arrow. Black dots represent individual trials (trials with incorrect responses removed). 


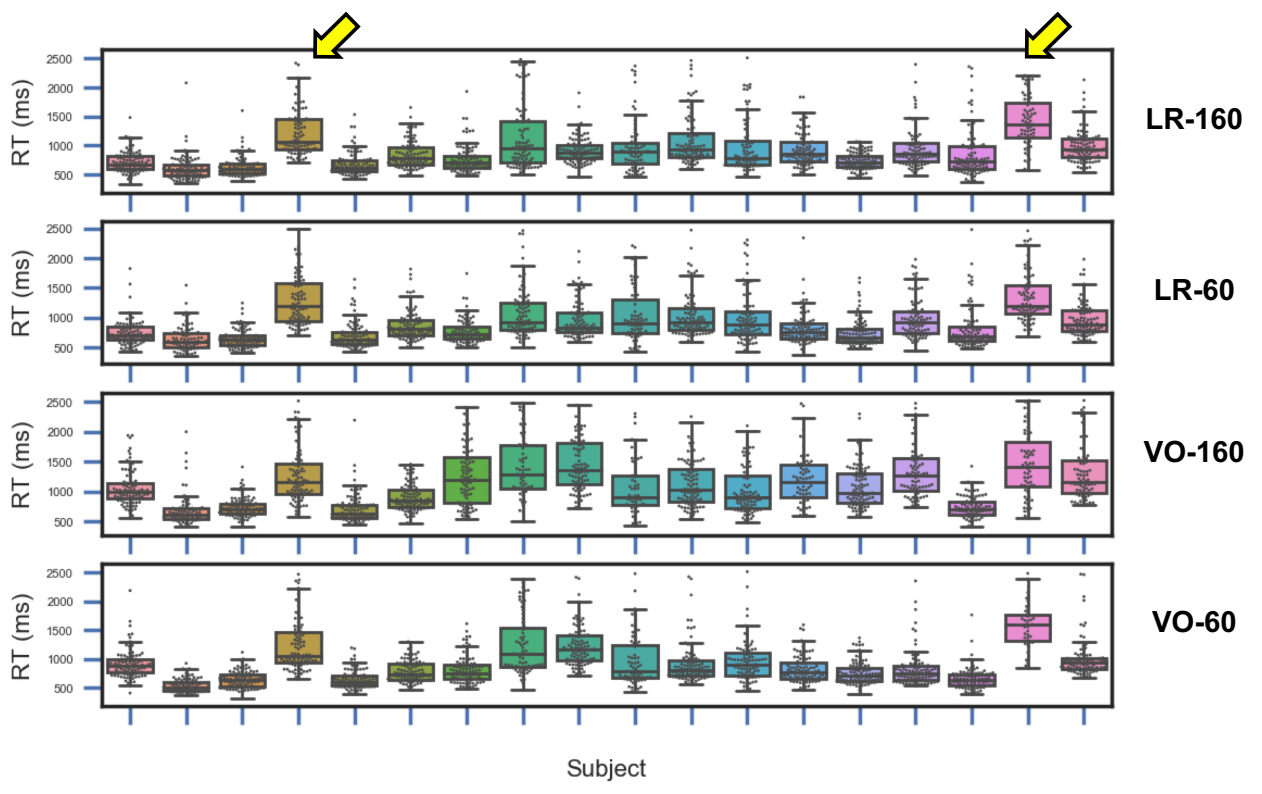

Individual control participant RT data are plotted below for each experimental condition, with the individuals removed highlighted with a yellow arrow. Black dots represent individual trials (trials with incorrect responses removed).

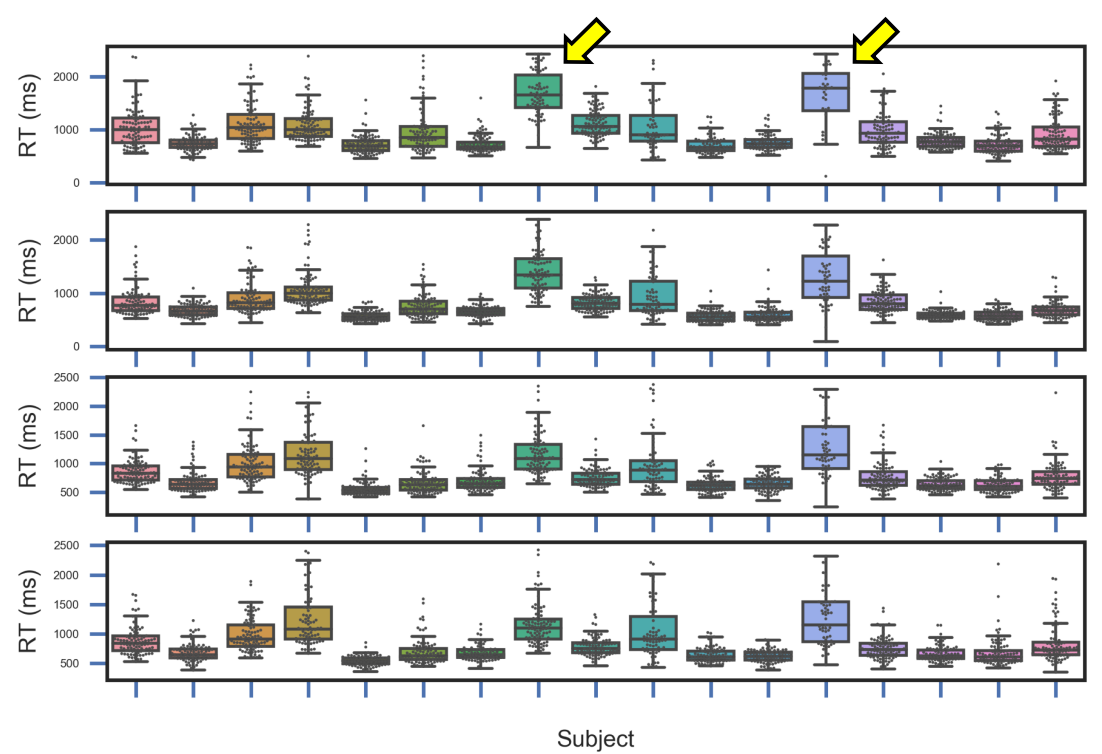

LR-160

LR-60

VO-160

Vo-60 


\section{Ethics Approval}

\section{Amendment request: $\mathbf{5 2 0 1 3 0 0 8 4 3}$}

Ethics Secretariat <ethics.secretariat@mq.edu.au>

Fri, Jul 7, 2017 at 2:08 PM

To: Jon Brock <jon.brock@mq.edu.au>

Cc: Paul Sowman <paul.sowman@mq.edu.au>, Robert Seymour <robert.seymour@students.mq.edu.au>

Hi Jon,

This is approved. Please take this email as confirmation of this approval.

RT

From: Jon Brock

Sent: Tuesday, 13 June 2017 7:14 PM

To: Ethics Secretariat <ethics.secretariat@mq.edu.au>

Cc: Paul Sowman <paul.sowman@mq.edu.au>; Robert Seymour <robert.seymour@students.mq.edu.au> Subject: Amendment request: 5201300843

Hello

Please find attached a request for amendments to Proposal 5201300843

This involves addition of PhD student, Robert Seymour, and his project, and removal of students who are no longer at Macquarie.

We also request that Assoc Prof Paul Sowman becomes the Chief Investigator as my position at Macquarie has now ended. Paul is Robert's primary supervisor (or will shortly be nominated as such) and is involved in the majority of projects already included in the proposal.

Please let us know if you need any further information.

Best wishes

Jon 


\section{Aston University}

Aston University Research Ethics Committee Aston University

Aston Triangle Birmingham

B4 7ET

Telephone +44 (0)1212043000

Fax +44(0)1212043696

Date: $18^{\text {th }}$ March 2016

Professor Klaus Kessler \& Professor Gina Rippon

Cc: Robert Seymour

Life and Health Sciences

Dear Klaus \& Gina,

\begin{tabular}{|l|l|}
\hline Study title: & $\begin{array}{l}\text { Investigating Sensory and Social Brain Connectivity in Autism using } \\
\text { Magnetoencephalography (MEG) }\end{array}$ \\
\hline REC REF: & Ethics Application \#574 \\
\hline
\end{tabular}

Confirmation of Ethical opinion

On behalf of the Committee, I am pleased to confirm a favourable opinion for the amendments listed in your email on $26^{\text {th }}$ February 2016 and the revised study documents.

\section{Approved Documents}

The final list of documents reviewed and approved by the Committee is as follows;

\begin{tabular}{|c|c|c|}
\hline Document & Version & Date \\
\hline Participant_info_FEB_2016_updated & $\begin{array}{l}\text { Attached to email dated } 15^{\text {th }} \\
\text { March }\end{array}$ & $15 / 03 / 2016$ \\
\hline General_consent_form_FEB & $\begin{array}{l}\text { Attached to email dated } 4^{\text {th }} \\
\text { March }\end{array}$ & $04 / 03 / 2016$ \\
\hline Glasgow_Sens_Qu PDF & $\begin{array}{l}\text { Attached to email dated } 4^{\text {th }} \\
\text { March }\end{array}$ & $04 / 03 / 2016$ \\
\hline AQ Doc & $\begin{array}{l}\text { Attached to email dated } 4^{\text {th }} \\
\text { March }\end{array}$ & $04 / 03 / 2016$ \\
\hline AQ_Adolescent Doc & $\begin{array}{l}\text { Attached to email dated } 4^{\text {th }} \\
\text { March }\end{array}$ & $04 / 03 / 2016$ \\
\hline $\begin{array}{l}\text { Email notifying UREC of proposed } \\
\text { amendments }\end{array}$ & & $26 / 02 / 2016$ \\
\hline
\end{tabular}




\title{
Aston University
}

\author{
Statement of compliance
}

The Committee operates in accordance with the Aston University Ethics policy and procedures:

http://www1.aston.ac.uk/registry/for-staff/regsandpolicies/ethics-policy-and-procedures/

\section{Reporting Requirements}

The details of the investigation will be placed on file. You should notify the Secretary of the University Ethics Committee of any adverse events which occur in connection with this study and/or which may alter its ethical consideration, and/or any difficulties experienced by the Volunteer subjects.

If you intend to make any future protocol amendments these must be approved by the Ethics Committee prior to implementation. You should also seek approval for any extension of the approved completion date.

Membership

The members of the University Ethics Committee present at the meeting are listed below:

- Include names of representatives who were present

REC Reference: Student Ethics Application \#574

Please quote this number on all correspondence

With the Committee's best wishes for the success of the project

Yours sincerely,

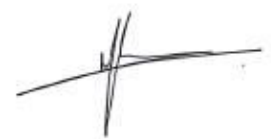

Dr Nichola Seare,

Chair of the Ethics Committee 